Printed April 1996

RECEIVED

MAY 151996

OSTI

\title{
Preliminary Data From an Instantaneous Profile Test Conducted Near the Mixed Waste Landfill, Technical Area 3, Sandia National Laboratories/New Mexico
}

Stephen C. Bayliss, Timothy J. Goering, Michael D. McVey, Warren R. Strong, Jerry L. Peace

\author{
Prepared by \\ Sandia National Laboratories \\ Albuquerque, New Mexico 87185 and Livermore, California 94550 \\ for the United States Department of Energy \\ under Contract DE-AC04-94AL85000
}

Approved for public release; distifbution is unlimited.

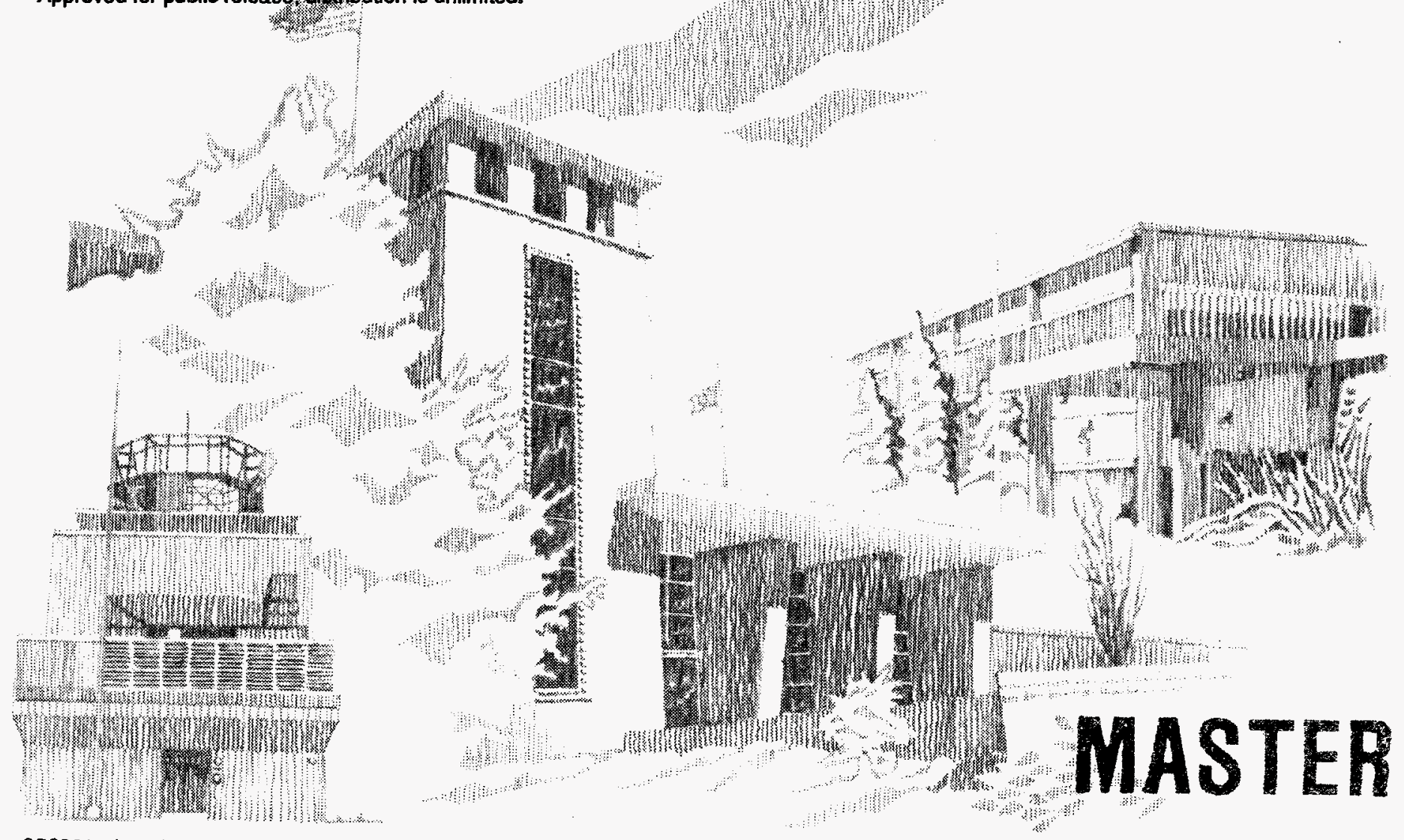


Issued by Sandia National Laboratories, operated for the United States Department of Energy by Sandia Corporation.

NOTICE: This report was prepared as an account of work sponsored by an agency of the United States Government. Neither the United States Government nor any agency thereof, nor any of their employees, nor any of their contractors, subcontractors, or their employees, makes any warranty, express or implied, or assumes any legal liability or responsibility for the accuracy, completeness, or usefulness of any information, apparatus, product, or process disclosed, or represents that its use would not infringe privately owned rights. Reference herein to any specific commercial product, process, or service by trade name, trademark, manufacturer, or otherwise, does not necessarily constitute or imply its endorsement, recommendation, or favoring by the United States Government, any agency thereof or any of their contractors or subcontractors. The views and opinions expressed herein do not necessarily state or reflect those of the United States Government, any agency thereof or any of their contractors.

Printed in the United States of America. This report has been reproduced directly from the best available copy.

Available to DOE and DOE contractors from

Office of Scientific and Technical Information

PO Box 62

Oak Ridge, TN 37831

Prices available from (615) 576-8401, FTS 626-8401

Available to the public from

National Technical Information Service

US Department of Commerce

5285 Port Royal Rd

Springfield, VA 22161

NTIS price codes

Printed copy: A05

Microfiche copy: A01 


\section{DISCLAMMBar}

Portions of this document may be illegible in electronic image products. Imsges are produced from the best available original document. 
SAND96-0813

Distribution

Unlimited Release

Category UC-903

Published April 1996

\title{
Preliminary Data From an Instantaneous Profile Test Conducted Near the Mixed Waste Landfill, Technical Area 3, Sandia National Laboratories/New Mexico
}

\author{
Stephen C. Bayliss \\ DanShar, Inc. \\ 1215 Chiquitos Rd. \\ Bosque Farms, NM 87068 \\ Timothy J. Goering and Michael D. McVey \\ GRAM, Inc. \\ 8500 Menaul Blvd., NE, Suite B-370 \\ Albuquerque, NM 87112 \\ Warren R. Strong and Jerry L. Peace \\ Sandia National Laboratories/New Mexico \\ Environmental Restoration Project \\ Albuquerque, NM 87185-5800
}

\begin{abstract}
This paper presents data from an instantaneous profile test conducted near the Sandia National Laboratories/New Mexico Mixed Waste Landfill in Technical Area 3. The test was performed from December 1993 through 1995 as part of the Environmental Restoration Project's Phase 2 RCRA Facility Investigation of the Mixed Waste Landfill. The purpose of the test was to measure the unsaturated hydraulic properties of soils near the Mixed Waste Landfill. The instantaneous profile test and instrumentation are described, and the pressure and moisture content data from the test are presented. These data may be useful for understanding the unsaturated hydraulic properties of soils in Technical Area 3 and for model validation, verification, and calibration.
\end{abstract}




\section{Acknowledgments}

The authors wish to thank Jackie Onsurez (GRAM, Inc.) for developing the TDR Trace Interpretation Program and for his assistance in setting up the instrumentation; and Mike Fort (INTERA, Inc.), Joe Fritts (GRAM Inc.), Chris Crawford (Technadyne), and Dr. Robert Knowlton (Sandia National Laboratories/New Mexico, Department 6603) for their assistance on this project. 


\section{Contents}

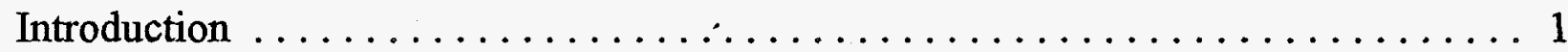

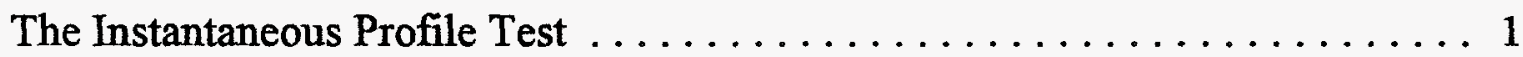

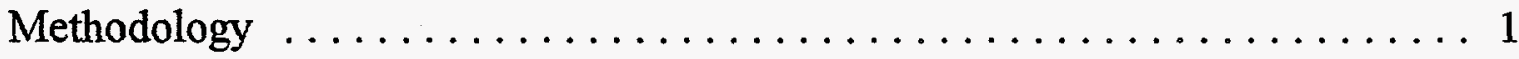

The Instantaneous Profile Test Site $\ldots \ldots \ldots \ldots \ldots \ldots \ldots \ldots \ldots \ldots \ldots \ldots$

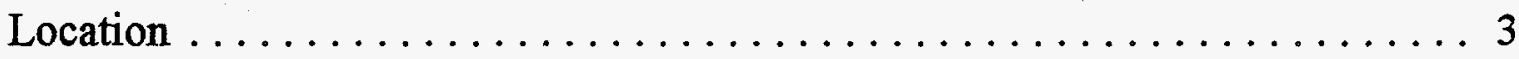

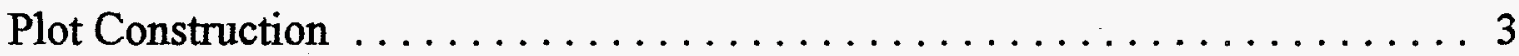

Installation of Instrumentation $\ldots \ldots \ldots \ldots \ldots \ldots \ldots \ldots \ldots$

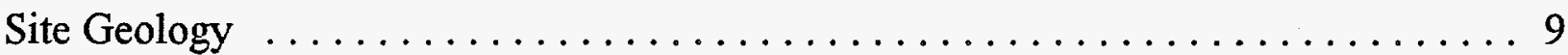

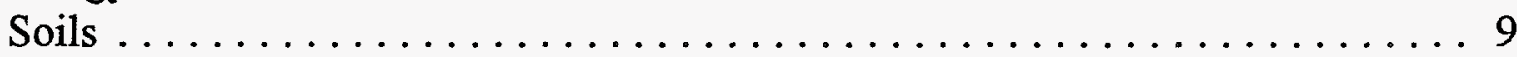

Subsurface Geology . . . . . . . . . . . . . . . . . . 12

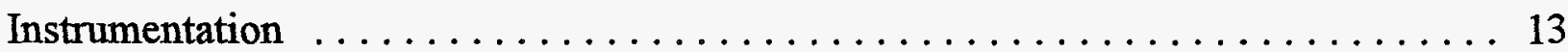

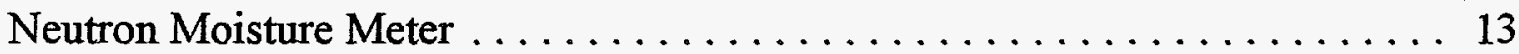

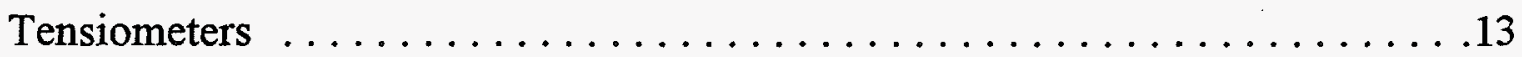

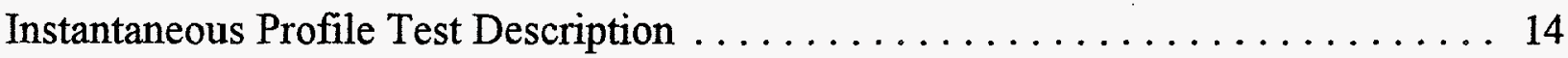

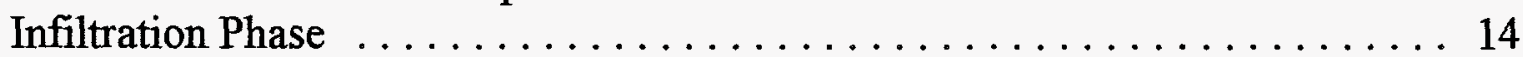

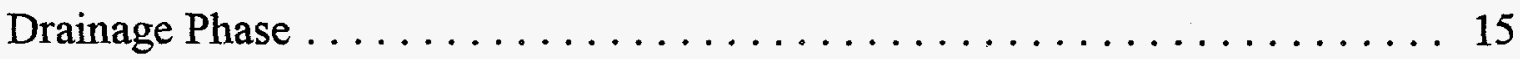

Test Results . . . . . . . . . . . . . . . . . . . . . . . . . . . 60

Neutron Moisture Meter Data . . . . . . . . . . . . . . . . 60

Tensiometer Data ............................. 63

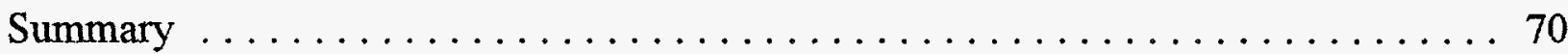

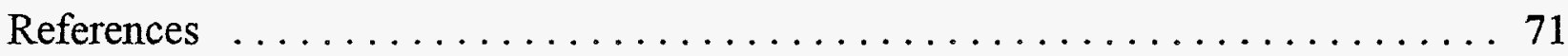




\section{Figures}

1 Instantaneous Profile Test Location $\ldots \ldots \ldots \ldots \ldots \ldots \ldots \ldots \ldots \ldots$

2 Instantaneous Profile Plot Configuration and Instrument Locations. ........ 5

3 Cross-section of Instantaneous Profile Plot. . . . . . . . . . . . . . 6

4 Cross-section of Instantaneous Profile Plot Showing Instrument Cluster C . . . . . 8

5 Instantaneous Profile Infiltration Test, Cumulative Flow. ........... 16

6 Instantaneous Profile Plot Head Levels During Infiltration Test. . . . . . . . . 17

7 Neutron Moisture Meter Data, SE Access Tube. ................. 61

8 Neutron Moisture Meter Data, Center Access Tube. . . . . . . . . . . . . 62

9 Soil Tension versus Time, $30 \mathrm{~cm}$ Depth. ..................... 64

10 Soil Tension versus Time, $60 \mathrm{~cm}$ Depth. . . . . . . . . . . . 65

11 Soil Tension versus Time, $90 \mathrm{~cm}$ Depth. . . . . . . . . . . . 66

12 Soil Tension versus Time, $120 \mathrm{~cm}$ Depth. . . . . . . . . . . . . 67

13 Soil Tension versus Time, $150 \mathrm{~cm}$ Depth. . . . . . . . . . . . . 68

14 Soil Tension versus Time, $180 \mathrm{~cm}$ Depth. . . . . . . . . . . . . . 69 


\section{Tables}

1 Textural Parameters for Madurez Loamy Fine Sand $\ldots \ldots \ldots \ldots \ldots \ldots$

2 Chemical and Textural parameters of Surface and Subsurface Soils at the Mixed Waste Landfill

3 Lithologic Descriptions of Core Samples Taken During Installation of the East Neutron Access Tube. . . . . . . . . . . . . . . . . . . . . . . 11

4 Neutron Moisture Meter Data. . . . . . . . . . . . . . . . . . . . . . . . . 18

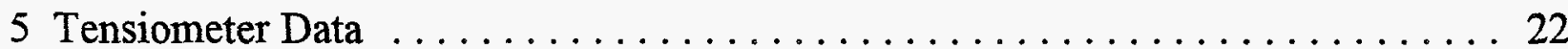




\subsection{Introduction}

\subsection{The Instantaneous Profile Test}

An instantaneous profile (IP) test was conducted approximately $150 \mathrm{~m}(500 \mathrm{ft})$ west of the Mixed Waste Landfill (MWL) in Technical Area 3 (TA-3) per the methods described by Hillel et al, (1972) and Watson (1966). The test was conducted from December 1993 and continued into fiscal year 1995 as part of the Sandia National Laboratories/New Mexico (SNL/NM) Environmental Restoration (ER) Project's Phase 2 RCRA Facility Investigation (RFI) of the MWL (SNL/NM, 1993). The objectives of the test were to measure the relationships between unsaturated hydraulic conductivity, pressure head, and moisture content for soil horizons to a depth of $1.8 \mathrm{~m} \mathrm{(6ft)}$ below ground surface (bgs).

The IP test was conducted for a period of nearly one and a half years, during which time an extensive data set was compiled. This paper presents the pressure head and moisture content data collected from December 1993 through 1994. These data were collected using a CPN Model 503DR neutron moisture meter, a Sentry 200 resonant frequency capacitance (RFC) probe, tensiometers, buried RFC probes, and time domain reflectometry (TDR) probes. The data set in this report is limited to data collected manually by neutron logging and to the corresponding soil tension values measured with tensiometers. An extensive data set was also collected using TDR and RFC probes, and may be presented in a later report.

The IP test consisted of flooding a $4.7 \mathrm{~m}$ by $4.7 \mathrm{~m}(15.5 \mathrm{ft}$ by $15.5 \mathrm{ft})$ soil plot with 20,300 liters ( 5494 gallons) of water over a period of 47 hours ( 2840 minutes). The wetting front was monitored using the instrumentation listed above. When the entire plot

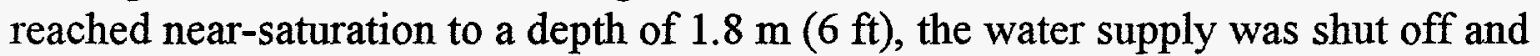
drainage from the IP plot was monitored. Due to entrapped air, it is unlikely that the entire soil profile was completely saturated at the end of the wetting phase, therefore, the term field-saturation will be used in this report.

\subsection{Methodology}

The IP test was originally proposed by Richards and Weeks (1953), Ogata and Richards (1957), and later by Watson (1966) as a method of measuring the relationship 
between water potential and hydraulic conductivity. This method is based upon monitoring the transient internal drainage of a profile. The relationship between water potential and hydraulic conductivity is determined by measuring the rate of drainage and water potential, and then solving a form of Richard's equation to obtain the unsaturated hydraulic conductivity as a function of water potential.

The IP method is superior for obtaining in situ measurements of unsaturated hydraulic conductivity as a function of moisture content, because it eliminates the disturbance of soil structure from sampling and the resulting changes in soil hydrologic parameters. This approach requires frequent, simultaneous measurements of soil tension and volumetric moisture content under transient drainage conditions. These data are used to obtain instantaneous values of the potential gradients and fluxes operating within the profile, and to calculate the resulting unsaturated hydraulic conductivities. 


\subsection{The Instantaneous Profile Test Site}

\subsection{Location}

The IP test was conducted in a non-contaminated area approximately $150 \mathrm{~m}(500$ $\mathrm{ft}$ ) west of the MWL in TA-3 (Figure 1).

\subsection{Plot Construction}

The IP site is a $4.73 \mathrm{~m}(15.5 \mathrm{ft})$ by $4.73 \mathrm{~m}(15.5 \mathrm{ft})$ soil plot surrounded by a 30 $\mathrm{cm}(1 \mathrm{ft}$ ) high concrete berm (Figure 2). Beneath the concrete berm on four sides of the plot are vertical no-flow boundary walls constructed of a bentonite-soil mixture to prevent horizontal flow from the plot, and to limit infiltration to one-dimensional vertical flow.

The no-flow boundary walls were constructed using a backhoe. Four interconnecting trenches were excavated to a depth of $1.8 \mathrm{~m}(6 \mathrm{ft})$ and a width of $30 \mathrm{~cm}$ $(1 \mathrm{ft}$ ). Bentonite was mixed with the excavated soil to form an approximate $10 \%$ bentonite-soil mixture. The bentonite-soil mixture was then placed back into the excavated trenches with the backhoe. A cross section of the IP plot showing the no-flow boundary walls is presented in Figure 3.

Once the no-flow boundary walls were constructed, a $30 \mathrm{~cm}(1 \mathrm{ft})$ wide by $30 \mathrm{~cm}$ $(1 \mathrm{ft}$ ) high concrete berm was constructed over the top of the no-flow boundary walls to retain ponded water in the soil plot.

\subsection{Installation of Instrumentation}

Five access tubes, consisting of $5 \mathrm{~cm}$ ( 2 in) Schedule 40 polyvinyl chloride (PVC) pipe, were sealed at the lower ends with rubber stoppers and installed vertically to a

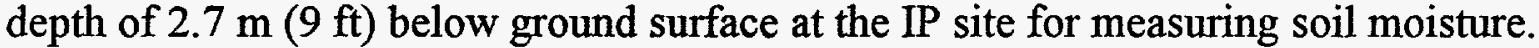
Three of the access tubes were installed inside of the IP plot and two access tubes were installed outside of the IP plot perimeter, adjacent to the concrete berm. Figure 2 shows the locations of the five access tubes. The access tubes located inside of the concrete berm were installed to measure soil moisture in the IP plot during the wetting and drying 


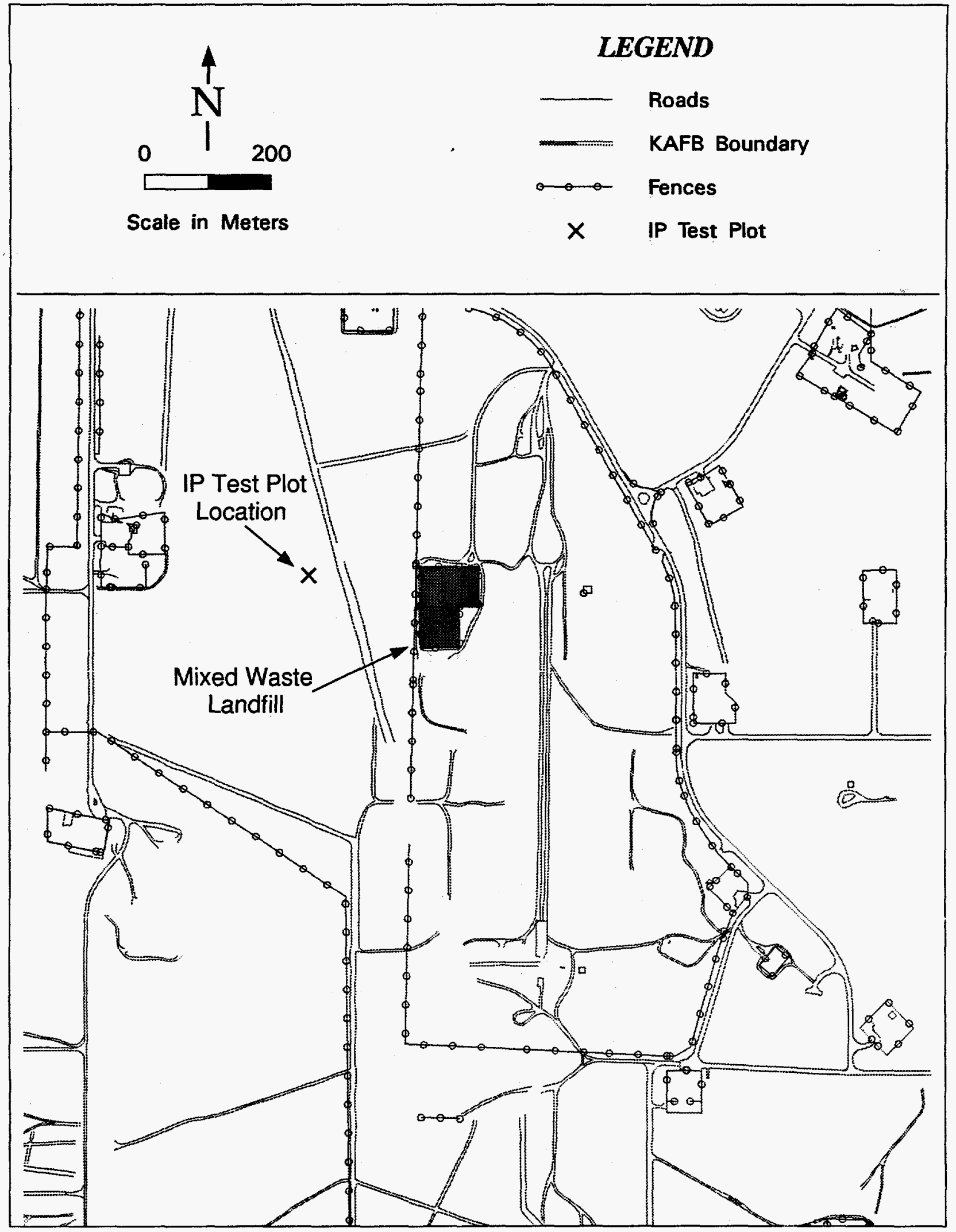

Figure 1. Instantaneous profile test plot location. 


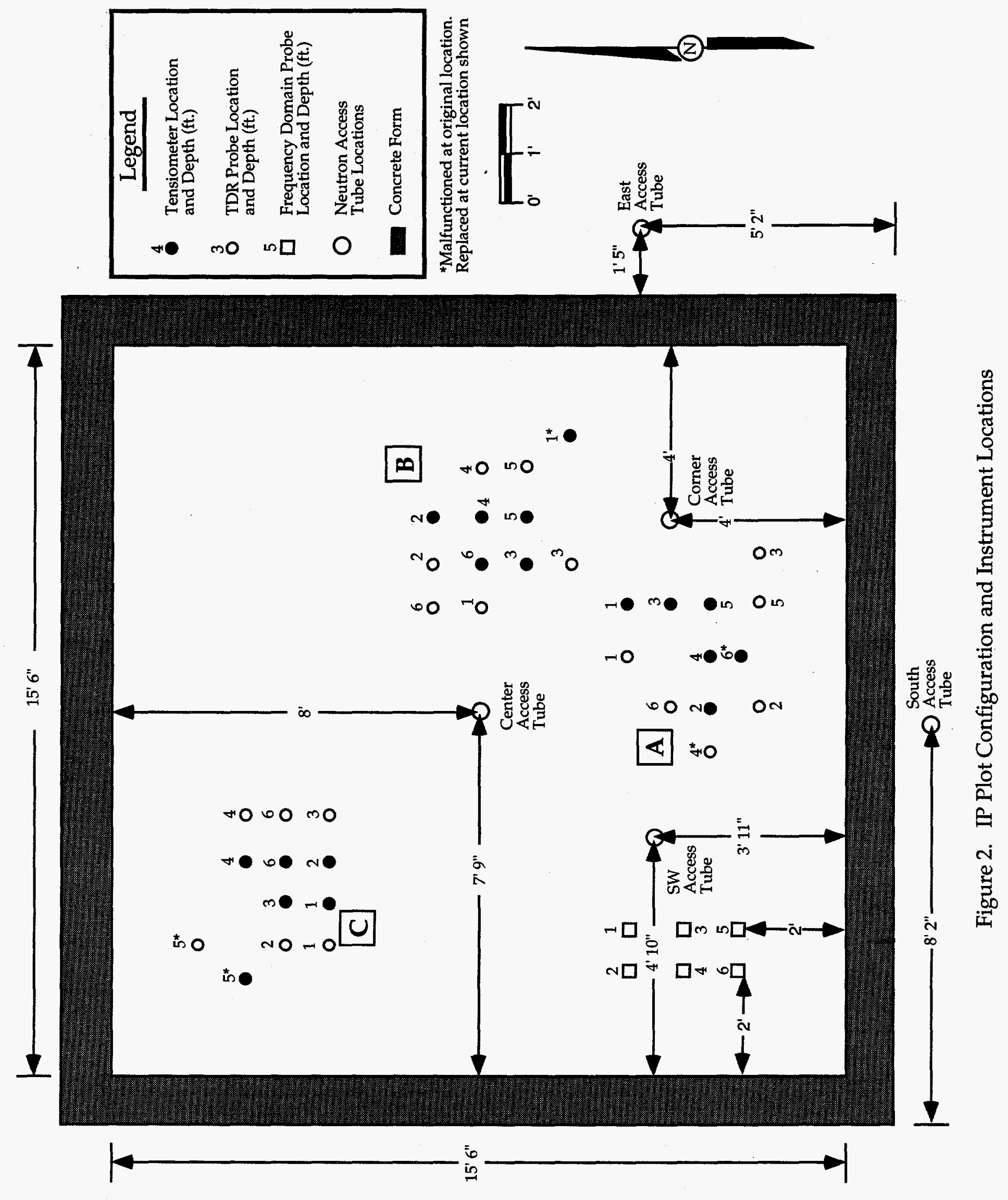




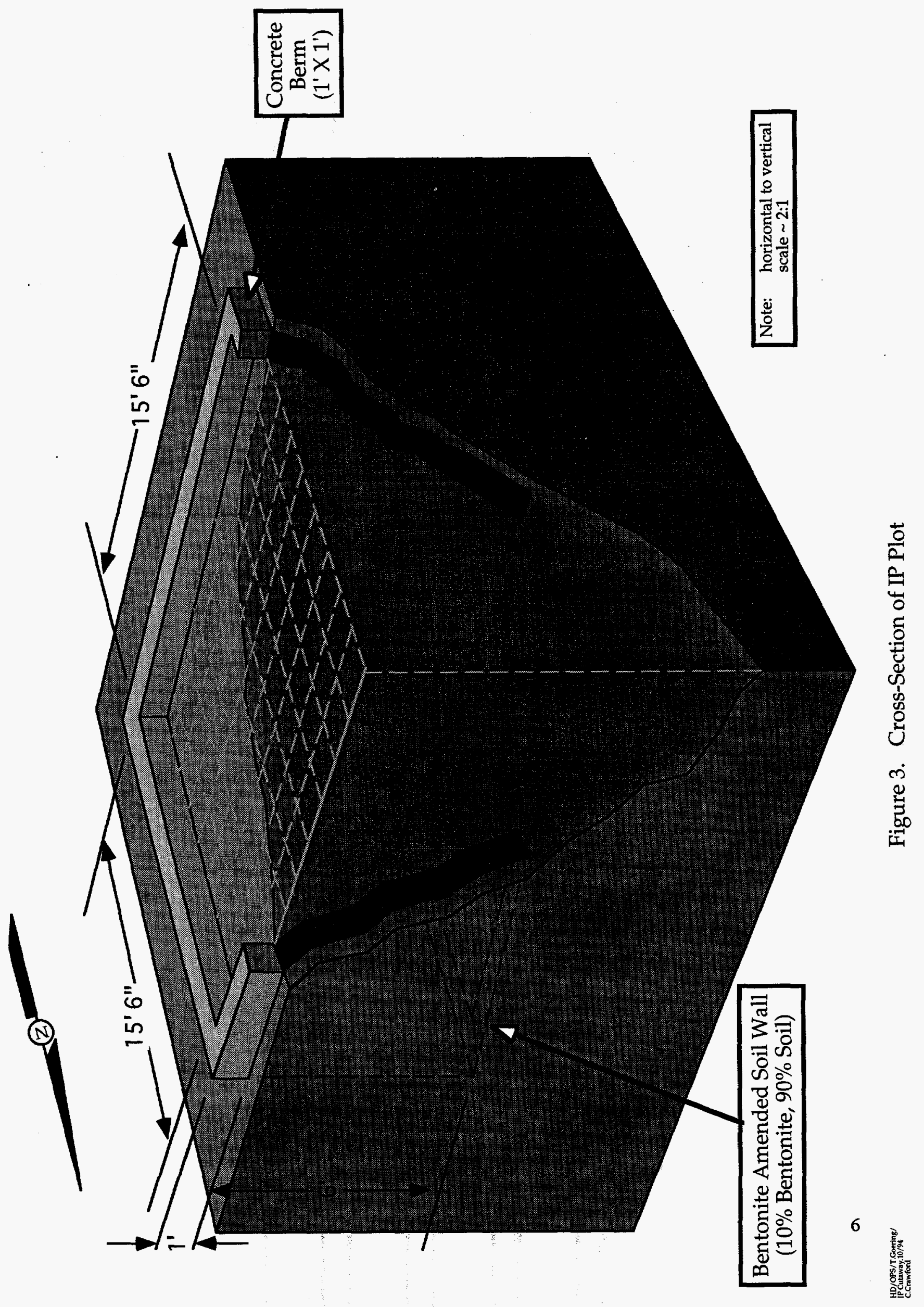


phases of the study. The two access tubes located outside of the concrete berm, on the east and south sides, were installed to determine if any leakage was occurring through the no-flow boundary walls.

A hand-held auger was used to drill the holes for installation of the access tubes. The center, southeast corner, and south perimeter access tubes were installed prior to initiating the experiment. The remaining access tubes were added five months after the start of the IP test for additional geotechnical and hydrologic data.

Six buried RFC probes were installed in the southwest corner of the IP plot (Figure 2) for measuring soil moisture content during the test. The RFC data is considered experimental, and is not presented in this report. Three instrument clusters (A, B, and C), consisting of six tensiometers and six TDR probes, were installed inside the IP plot at depths ranging from $30 \mathrm{~cm}(1 \mathrm{ft})$ to $1.8 \mathrm{~m}(6 \mathrm{ft})$ below ground surface. The tensiometers and TDR probes were installed to measure soil tension and soil moisture at $30 \mathrm{~cm}(1 \mathrm{ft})$ vertical increments within the IP plot. The locations of the three instrument clusters and the specific location and depth of each tensiometer and TDR probe within each instrument cluster are shown in Figure 2. A cross section of the IP plot showing Instrument Cluster $\mathrm{C}$ is presented in Figure 4.

The TDR probes were installed by first auguring to the desired depth and then inserting the probes into undisturbed soil. The boreholes were then backfilled with the native soil excavated from the borehole during auguring.

Access holes for the tensiometers were drilled to the desired depth using a truckmounted hydraulic geoprobe. The tensiometers were then inserted into the holes and covered by backfilling with native soil around each tube. The tensiometers and TDR probes were installed adjacent to each other for paired readings of tension and moisture content. 


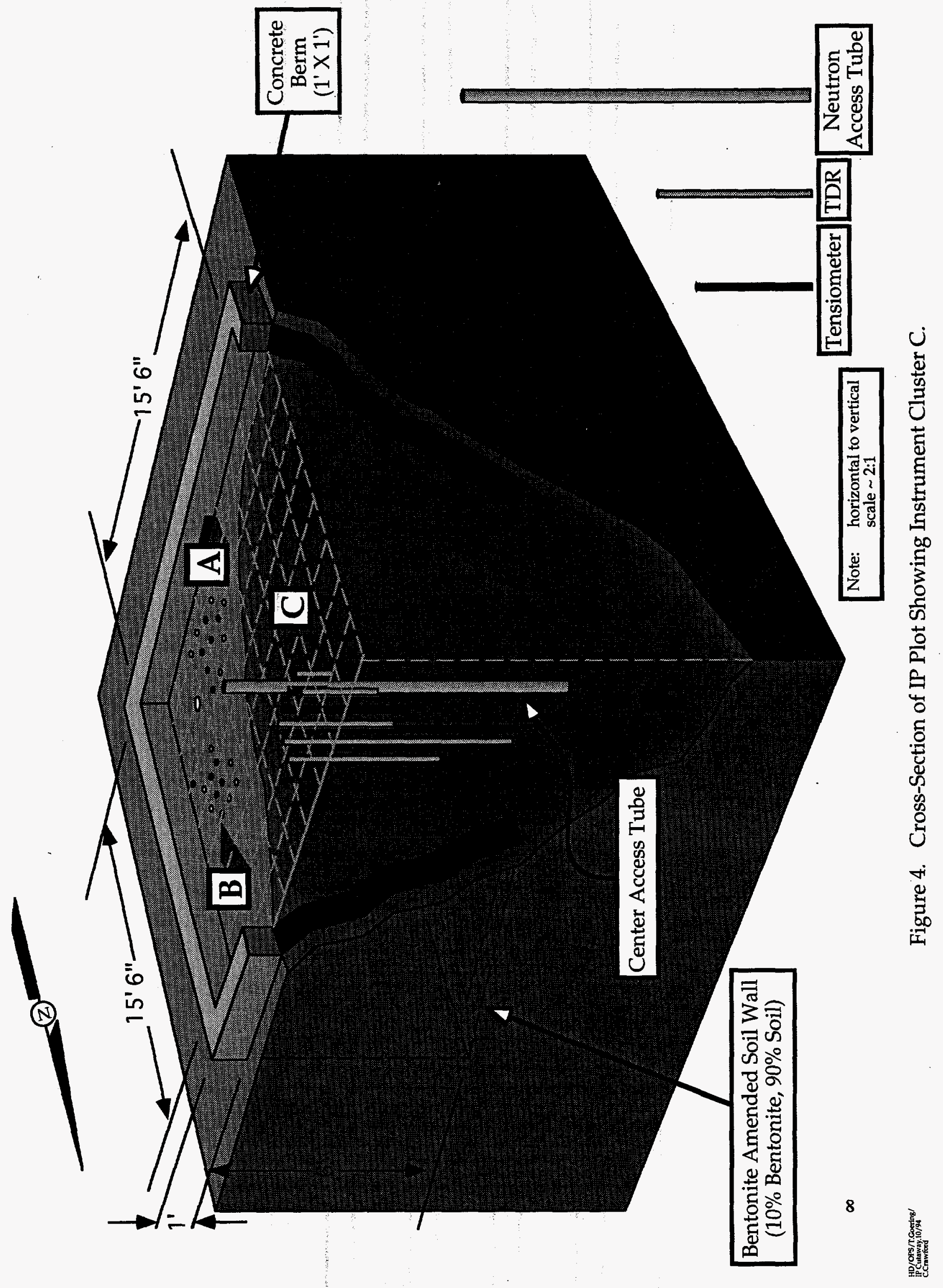




\subsection{Site Geology}

\subsection{Soils}

Soils in the area of the MWL and the IP Test Site are of the Madurez Loamy Fine Sand Series (USDA-SCS, 1977). These gently undulating soils are derived from old alluvium modified by wind and occur on slopes of one to five percent. Madurez Fine Sandy Loam soils are also prevalent. These soils are well-drained and runoff is slow. The soils are moderately alkaline throughout and calcareous below a depth of $33 \mathrm{~cm}(13$ in). Irregular zones of higher calcareous content can be present throughout the soil profile. A typical Madurez Loamy Fine Sand soil profile consists of a brown, fine sandy loam at depths greater than $43 \mathrm{~cm}$ (17 in). The textural parameters for the Madurez Loamy Fine Sand Series are shown in Table 1, and chemical and textural parameters of surface and subsurface soils at the MWL are shown in Table 2.

During installation of the east perimeter access tube (Figure 2), core samples were collected for lithologic description. The lithologic descriptions are presented in Table 3.

Table 1. Textural Parameters for Madurez Loamy Fine Sand ${ }^{a}$

\begin{tabular}{ccl}
\hline $\begin{array}{c}\text { Depth } \\
\text { (In.) }\end{array}$ & $\begin{array}{c}\text { Depth } \\
(\text { Cm })\end{array}$ & $\begin{array}{l}\text { Depth }^{\text {b }} \\
(\text { USDA) }\end{array}$ \\
\hline $0-9$ & $0-23$ & LoFSa \\
$9-21$ & $23-53$ & SaCLLo \\
$21-60$ & $53-152$ & SaLo \\
\hline
\end{tabular}

aSource: USDA-SCS, 1977

${ }^{\mathrm{b}} \mathrm{Lo}=\operatorname{loam}(\mathrm{y}), \mathrm{F}=\mathrm{Fine}, \mathrm{Cl}=\mathrm{Clay}, \mathrm{Sa}=\mathrm{Sand}(\mathrm{y})$ 
Table 2. Chemical and Textural Parameters of Surface and Subsurface Soils at the Mixed Waste Landfilla

\begin{tabular}{|c|c|c|c|c|c|c|c|c|}
\hline $\begin{array}{c}\text { Depth } \\
\text { (In.) }\end{array}$ & $\begin{array}{c}\text { EC } \\
(\mathrm{mmhos} / \mathrm{cm})^{\mathrm{b}}\end{array}$ & $\mathrm{pH}$ & $\mathrm{CaCO}_{3}$ & $\begin{array}{c}\text { Organic } \\
\text { Matter } \\
(\%)\end{array}$ & $\begin{array}{c}\mathrm{CEC} \\
(\mathrm{meq} / 100 \mathrm{~g})\end{array}$ & $\begin{array}{c}\text { Sand } \\
(\%)\end{array}$ & $\begin{array}{c}\text { Silt } \\
\text { (\%) }\end{array}$ & $\begin{array}{c}\text { Clay } \\
(\%)\end{array}$ \\
\hline $0-11$ & 3.45 & 8.27 & 0.5 & 0.2 & 6.51 & 82.58 & 8.95 & 8.47 \\
$11-18$ & 15.53 & 7.86 & 3.9 & 0.2 & 8.18 & 83.28 & 7.13 & 9.59 \\
$18-26$ & 13.79 & 7.76 & 5.5 & 0.1 & 7.27 & 85.9 & 6.17 & 7.93 \\
$26-38$ & 19.7 & 7.63 & 11.3 & 0.27 & 5.76 & 86.69 & 8.43 & 4.88 \\
$38-62$ & 27.81 & 7.48 & 21.4 & 0.07 & 6.52 & 69.49 & 25.05 & 5.46 \\
$79-83$ & 4.6 & 8.25 & 8.3 & 0 & 5.88 & 86.97 & 9.91 & 3.12 \\
$83-89$ & 4.31 & 8.17 & 6.3 & 0 & 6.8 & 84.23 & 9.57 & 6.2 \\
$89-99$ & 5.43 & 7.93 & 10.8 & 0 & 9.15 & 75.19 & 14.59 & 10.22 \\
$99-117$ & 6.34 & 7.96 & 16.8 & 0 & 10.1 & 76.75 & 9.22 & 14.03 \\
$117-130$ & 5.47 & 7.99 & 10.9 & 0.07 & 9.17 & 62.42 & 26.65 & 10.93 \\
$130-137$ & 3.46 & 8.1 & 8.3 & 0 & 7.41 & 70.39 & 23.18 & 6.43 \\
$137-151$ & 4.31 & 8.04 & 8.6 & 0.03 & 7.66 & 71.14 & 19.28 & 9.58 \\
$151-170$ & 5.78 & 7.94 & 11.8 & 0 & 10.93 & 49.58 & 40.99 & 9.43 \\
$170-184$ & 5.43 & 7.93 & 9.1 & 0.03 & 7.57 & 59.22 & 27.16 & 13.62 \\
$184-194$ & 3.31 & 8.08 & 4.2 & 0 & 7.88 & 86.06 & 4.2 & 9.74 \\
$194-216$ & 1.91 & 8.31 & 4.4 & 0 & 5.34 & 91.51 & 2.17 & 6.32 \\
$216-233$ & 3.81 & 8.02 & 18.9 & 0 & 4.24 & 85.2 & 12.18 & 2.62 \\
$233-253$ & 3.71 & 8 & 7.9 & 0 & 5.31 & 85.11 & 6.31 & 8.58 \\
$252-269$ & 2.84 & 8.13 & 5.3 & 0 & 4.35 & 91.95 & 2.39 & 5.66 \\
$269-282$ & 1.96 & 8.45 & 3.5 & 0 & 2.83 & 92.56 & 3.22 & 4.22 \\
$282-302$ & 2.28 & 8.2 & 7.4 & 0 & 3.59 & 91.47 & 3.97 & 4.56 \\
$302-318$ & 1.27 & 8.18 & 9.9 & 0 & 3.38 & 89.51 & 5.67 & 4.82 \\
$318-347$ & 1.35 & 8.36 & 3.5 & 0.03 & 5.6 & 86.93 & 4.92 & 8.15 \\
$347+$ & 1.74 & 8.33 & 6.5 & 0.03 & 3.68 & 92.91 & 1.98 & 5.11 \\
& & & & & & & & \\
\hline
\end{tabular}

Source: SNL, 1980. Research by Peter Wierenga (New Mexico State University) on the MWL (unpublished report).

${ }^{\circ} \mathrm{EC}=$ Electrical Conductivity, mmhous/cm=millimhos per centimeter. ${ }^{\circ} \mathrm{CEC}=$ Cation exchange capacity, meq=milliequivalent. 
Table 3. Lithologic Descriptions of Core Samples Taken During Installation of the East Neutron Access Tube.

$0 "-35 "$ : Sand with silt; sand - fine to very fine grained with $2 \%$ coarse to very coarse grains, arkosic, subangular to subrounded; granules $5 \%$ and $\leq 4 \mathrm{~mm}$, subangular to subrounded, predominately limestone, some quartzite; caliche granules $<2 \%$ and $\leq 4$ $\mathrm{mm}$. Pebbles $4 \%$ and $\leq 40 \mathrm{~mm}$, predominately $(80 \%) \leq 20 \mathrm{~mm}$, subangular to subrounded, limestone and quartzite (limestone approximately $85 \%$, quartzite approximately $15 \%$ ). Sand is loose and moderately to well-sorted, dry, strong reaction with $\mathrm{HCL}$, brown to light brown $5 \mathrm{YR} 5 / 6$.

37 " -43 " : Silty sand with caliche; sand - fine to very fine grained with $\leq 1 \%$ coarse to very coarse grains, arkosic, subangular to subrounded, moderately to well-sorted; granules $5 \%$ and $\leq 4 \mathrm{~mm}$, subangular to subrounded, quartz and limestone, pebbles $3 \%$ and $\leq 4 \mathrm{~mm}$, limestone and quartz. Sand is powdery with fragments moderately compacted, weakly to moderately cemented, moderate caliche as matrix, very strong reaction with $\mathrm{HCL}$, light creamy brown $5 \mathrm{YR} 7 / 2$.

49"-99" : Silty sand with caliche; same as 37 "-43" except fragments are moderately to well cemented, caliche as matrix.

$100 "-111 "$ : Silty sand with caliche; sand - fine to very fine grained with $2 \%$ coarse to very coarse grains, arkosic, subangular to subrounded, moderately to well-sorted; granules $3 \%$ and $\leq 4 \mathrm{~mm}$, subangular to subrounded, quartz and limestone, pebbles $5 \%$ and $\leq 4 \mathrm{~mm}$, subangular to subrounded, limestone and quartz. Sand is powdery with fragments weakly to moderately compacted, very weakly cemented, moderate to little caliche as matrix, dry, moderate reaction with $\mathrm{HCL}$, light brown 5YR5/4. 


\subsection{Subsurface Geology}

The IP site and the MWL are underlain exclusively by the Santa Fe Group deposits. Sedimentary stratification beneath the IP site and the MWL is moderately to well developed and some graded bedding may occur. Beds can vary in thickness from a few centimeters to several meters. Bedding is often lenticular with limited aerial extent. However, channel beds can extend downdip for long distances, and similarly, thin sheets of fine sediments lain by flood deposits can extend over significant areas. Caliche layers are commonly formed on subaerially-exposed surfaces. Samples collected during drilling at the MWL indicate little correlation of individual sedimentary layers between wells and borings. However, the coarse-grained sediments near the surface of the MWL generally grade to fine-grained sediments with depth. 


\subsection{Instrumentation}

Volumetric moisture contents were measured with a neutron moisture meter, RFC probes, and TDR probes. Soil water tension was measured with 1-bar porous cup tensiometers. Multiple instruments were used only for the purpose of side-by-side comparison of available technologies. The neutron moisture data and tensiometer data were used to calculate unsaturated hydraulic conductivities within the IP plot. These data are presented in Tables 4 and 5 . This report uses only the tensiometer and neutron moisture meter data; hence only these instruments are described below.

\subsection{Neutron Moisture Meter}

Volumetric soil moisture data were obtained using a CPN Model 503DR neutron probe. The probe was manually lowered into the PVC access tubes and readings were collected at $30 \mathrm{~cm}(1 \mathrm{ft})$ intervals. Moisture data were recorded manually as raw counts from the neutron probe digital display. The raw counts were later converted to volumetric moisture using a site-specific calibration equation. This site-specific equation was determined using standard field calibration methods. The access tube locations are shown in Figure 2.

\subsection{Tensiometers}

Soil water tension data were obtained using 1-bar porous cup tensiometers developed by Soil Measurement Systems, Inc. The tensiometer cups are approximately 5 $\mathrm{cm}(2 \mathrm{in})$ long and $2.5 \mathrm{~cm}$ (1 in) in diameter, and are connected to the ground surface by a water-filled extension pipe. Tension values were monitored and recorded using pressure transducers connected to a Campbell CR7 electronic data logger. The transducers were calibrated in SNL/NM's Geohydrology Laboratory prior to installation in the field. Transducer output was calibrated against negative water pressure in units of bars. 


\subsection{Instantaneous Profile Test Description}

The IP test was initiated at 10:40 AM on December 14, 1993. The test was conducted in two phases: an infiltration phase, and a drainage phase.

\subsection{Infiltration Phase}

During the infiltration phase, water was applied to the test plot and allowed to infiltrate into the soil profile until the entire test volume $(4.73 \mathrm{~m}$ by $4.73 \mathrm{~m}$ by $1.8 \mathrm{~m})$ ( $15.5 \mathrm{ft}$ by $15.5 \mathrm{ft}$ by $6 \mathrm{ft}$ ) approached near-saturated conditions. The cumulative flux of water applied was continuously monitored, and the advance of the wetting front was observed with the instrumentation in place and the neutron moisture meter.

During the infiltration phase of the IP test, 20,300 liters (5494 gallons) of water were distributed over the test plot through a flow distribution system. The flow distribution system maintained an average constant head of $5.2 \mathrm{~cm}(2$ in) over the test plot, using a water-level sensitive switch wired to a solenoid and an electric pump. Water was distributed evenly across the IP test plot through a network of approximately $12.2 \mathrm{~m}(40 \mathrm{ft})$ of porous tubing, open at the end.

The plot was initially flooded at a constant pumping rate of 0.40 liters per second (6.3 gpm) until an average head of $5.2 \mathrm{~cm}$ ( 2 in) was maintained over the center of the plot. Once a constant head had been reached, the pump was automatically cycled on and off to maintain this head level, using the electronic switching device discussed above.

Figure 5 presents the cumulative flow added to the IP test plot, and Figure 6 presents instantaneous flow rates during the wetting portion of the test. Flow rates were somewhat irregular at first due to initial problems with the solenoid switch and water level detector. Once these problems were resolved, the flow rate remained uniform.

The advance of the wetting front was monitored by neutron logging and by collecting RFC measurements every half hour for the first 6 hours of the infiltration test, then hourly for the next 19 hours, then every four to nine hours until the drainage phase of the test began. Tensiometers and TDR probes were electronically monitored and their data were recorded at more frequent intervals, ranging from 10 to 30 minutes. 
The moisture data collected from the neutron logging, and the corresponding tension data collected from the tensiometers are presented in Tables 4 and 5 , respectively. Collection of the tensiometer data was computer controlled, allowing for quick tensiometer data acquisition, while neutron moisture data was manually collected. The remaining data (TDR, RFC probes, etc.) has been archived and may be presented in a later paper.

\subsection{Drainage Phase}

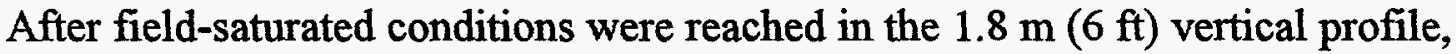
flooding was terminated and the drainage phase of the test began. The drainage phase was initiated on December 16, 1994 at 10:00 AM, 47 hours after the IP test plot was first flooded.

After the pump was turned off, head levels in the test plot dropped rapidly, as shown in Figure 6. Once the standing water in the plot had drained, the plot was covered with Visqueen ${ }^{\mathrm{TM}}$ and an overlying vinyl tarp to eliminate evaporation.

Neutron logging and RFC measurements were manually collected for the first 32 hours of the drainage phase of the test. Readings were then collected every 4 hours for the next 48 hours of the test. Thereafter, the frequency of readings was diminished to 8 hours, 12 hours, daily, weekly, and, eventually monthly, as the rate of drainage from the plot decreased asymptotically. 


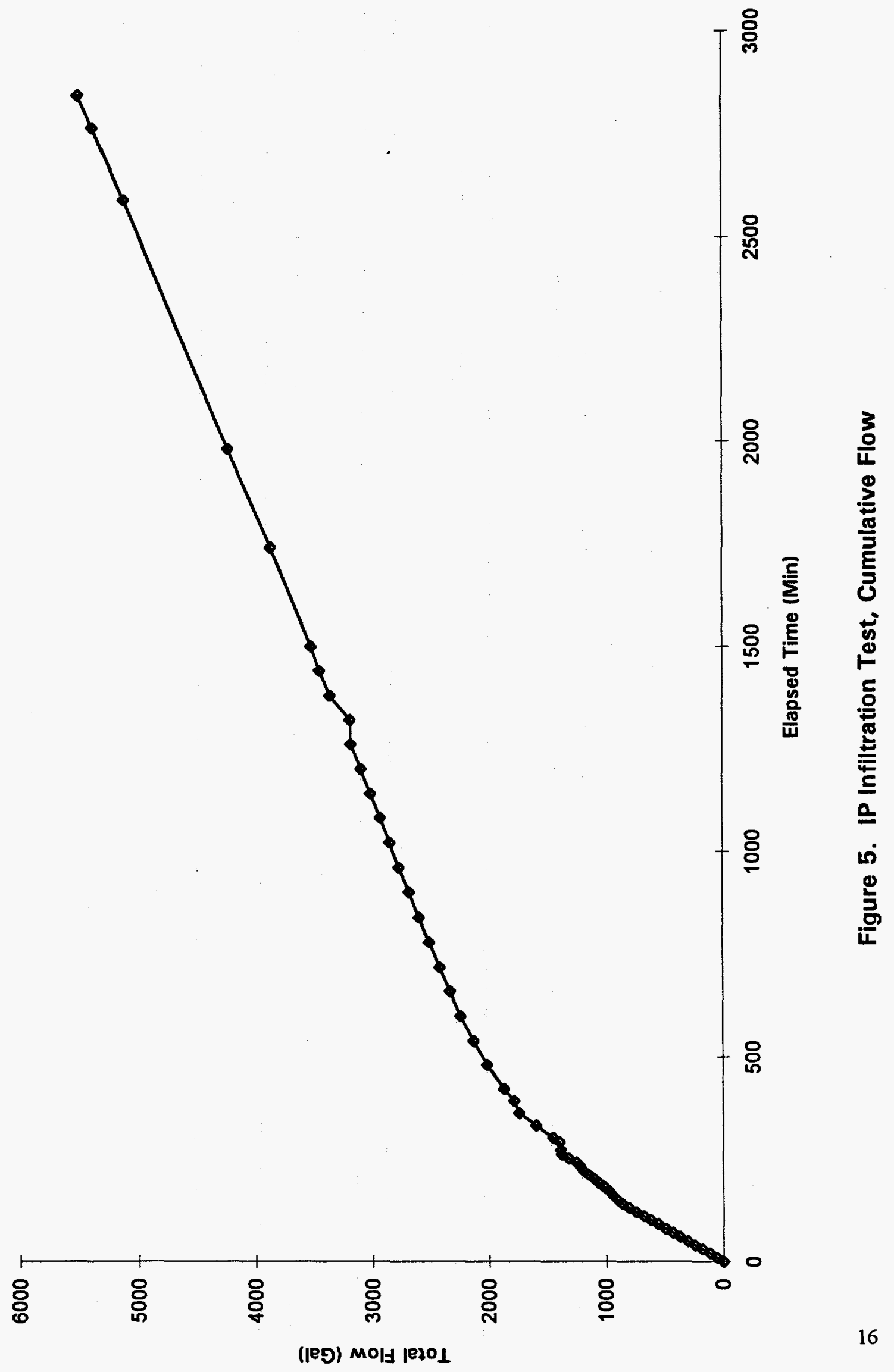




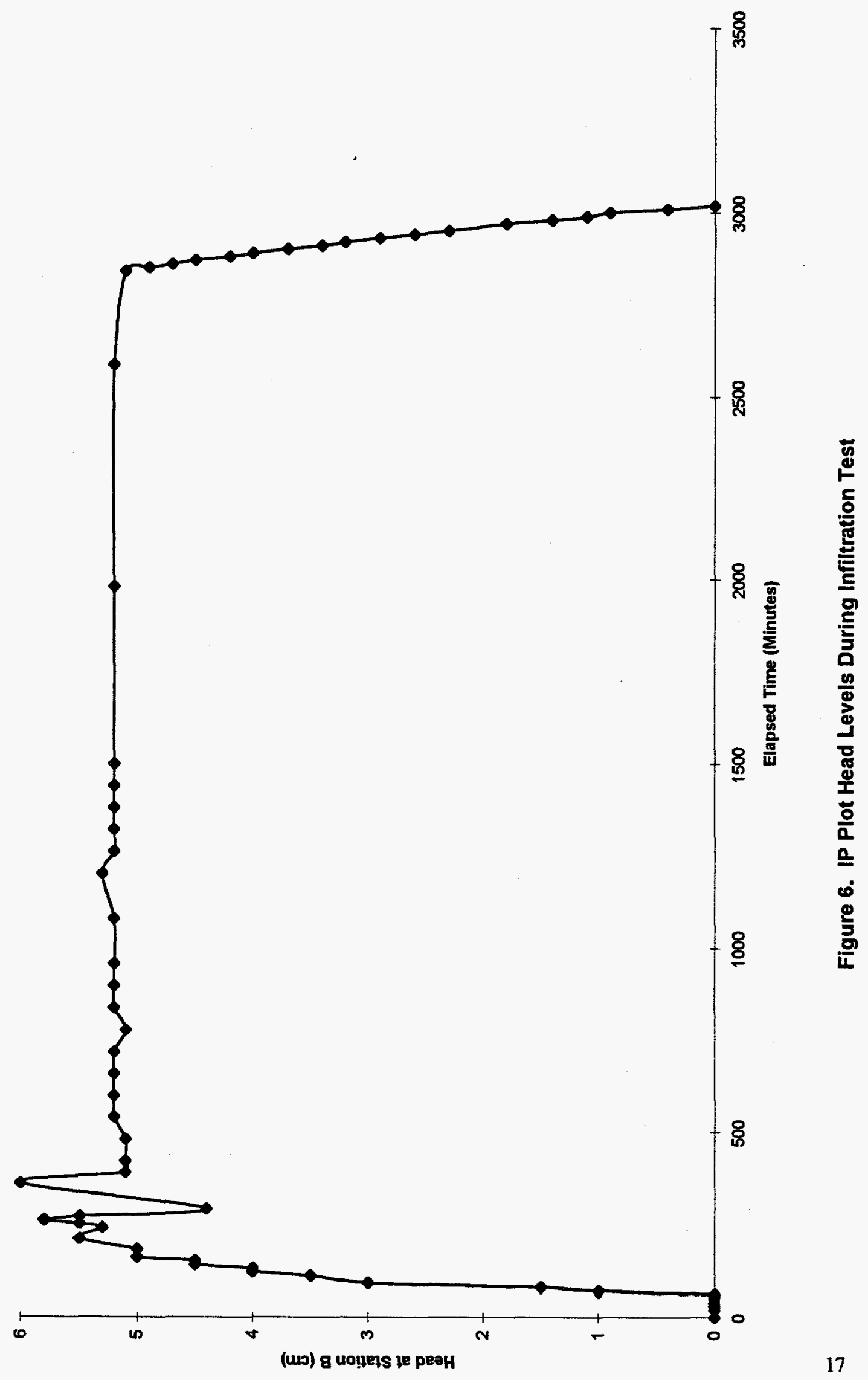


Table 4. Neutron Moisture Meter Data.

\begin{tabular}{|c|c|c|c|c|c|c|c|c|c|c|c|c|c|c|c|c|c|}
\hline \multicolumn{18}{|c|}{ Neutron Molsture Meter Data. } \\
\hline & & & & & & & & & & & & & & & & & \\
\hline & & & \multicolumn{5}{|c|}{ Access Tube, Southeast Comer of IP site } & & & & & \multicolumn{5}{|c|}{ Access Tube, Center of IP Site } & \\
\hline & & & & & & & & & & & & & & & & & \\
\hline & & Test & $\begin{array}{l}\text { Volumetric } \\
\text { Molsture } \\
\text { Content \% }\end{array}$ & $\begin{array}{l}\text { Volumetric } \\
\text { Moisture } \\
\text { Content } \%\end{array}$ & $\begin{array}{c}\text { Volumetric } \\
\text { Moisture } \\
\text { Content } \%\end{array}$ & $\begin{array}{l}\text { Volumetric } \\
\text { Molsture } \\
\text { Contenty }\end{array}$ & $\begin{array}{l}\text { Volumetric } \\
\text { Molsture } \\
\text { Content \% }\end{array}$ & $\begin{array}{l}\text { Volumetric } \\
\text { Moisture } \\
\text { Content } \mathrm{P}\end{array}$ & & & Test & \begin{tabular}{c|} 
Volumetric \\
Molsture \\
Content $\$$
\end{tabular} & $\begin{array}{l}\text { Volumetric } \\
\text { Molature } \\
\text { Content } y\end{array}$ & $\begin{array}{l}\text { Volumetric } \\
\text { Molsture } \\
\text { Content } \%\end{array}$ & $\begin{array}{l}\text { Volumetric } \\
\text { Molsture } \\
\text { Content } \%\end{array}$ & $\begin{array}{l}\text { Volumotrle } \\
\text { Molsture } \\
\text { Content } \%\end{array}$ & $\begin{array}{l}\text { Volumetric } \\
\text { Molsture } \\
\text { Content } \%\end{array}$ \\
\hline Date & Time & Days' & $60 \%$ & 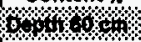 & on & 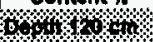 & $0.10 \%$ & $0+1 \%$ w & Date & Time & Days' & $0 \%$ on & of 10 & 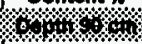 & $x+1 \%$ & 10 & $1 \% 1 \%$ \\
\hline 194 & $7: 30$ & -2.104 & 14.8 & 8.9 & 8.6 & 12.8 & 11.9 & 8.3 & \multirow{3}{*}{$\sqrt{17} 19$} & $7: 35$ & -2.101 & 11.8 & 8.0 & 11.5 & 15.1 & 13.2 & 10.7 \\
\hline \multirow[t]{7}{*}{ 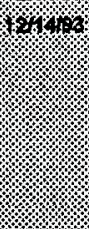 } & 11:10 & -1.951 & 14.7 & 8.8 & 8.8 & 13.0 & 11.8 & 8.2 & & $11: 18$ & -4.946 & 12.2 & 9.0 & 12.1 & 15.3 & 13.5 & 10.9 \\
\hline & $11: 47$ & -1.926 & 16.9 & 8.1 & 9.0 & 13.2 & 11.8 & 9.1 & & $11: 40$ & -1.931 & 14.1 & 9.0 & 11.6 & 15.5 & 13.2 & 10.8 \\
\hline & $12: 10$ & -1.910 & 21.7 & 9.0 & 8.5 & 13.0 & 11.5 & 8.6 & \multirow[t]{7}{*}{ 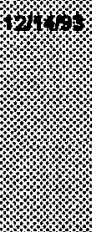 } & $12: 16$ & -1.906 & 24.9 & 8.7 & 11.7 & 15.3 & 13.1 & 10.7 \\
\hline & $12: 40$ & -1.889 & 29.5 & 9.1 & 11.7 & 15.6 & 13.7 & 11.0 & & $12: 40$ & $-1,889$ & 29.2 & 9.3 & 8.8 & 12.5 & 11.5 & 9.3 \\
\hline & 13:10 & $-1,868$ & 31.7 & 9.1 & 8.4 & 13,1 & 11.6 & 9.4 & & $13: 10$ & -4.868 & 31.1 & 10.6 & 12.0 & 15.9 & 13.2 & 10.6 \\
\hline & $13: 40$ & -1.847 & 32.1 & 13.8 & 8.4 & 13.1 & 11.4 & 8.9 & & $13: 40$ & -1.847 & 31.6 & 15.6 & 12.1 & 15.6 & 13.1 & 10.7 \\
\hline & 14:10 & -1.826 & 32.2 & 19.2 & 8.7 & 12.9 & 11.3 & 9.2 & & 14:10 & -4.826 & 32.1 & 29.0 & 12.1 & 15.5 & 13.0 & 10.8 \\
\hline \multirow[t]{7}{*}{ 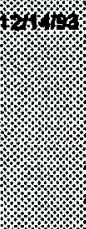 } & $14: 40$ & -1.806 & 32.7 & 31.7 & 9.0 & 12.8 & 11.8 & 9.4 & & $14: 40$ & -1.806 & 31.8 & 31.3 & 12.6 & 15.6 & 12.8 & 10.7 \\
\hline & $15: 10$ & -1.785 & 32.4 & 33.4 & 9.4 & 12.6 & 11.6 & 8.9 & & $15: 10$ & -4.785 & 32.5 & 31.3 & 15.5 & 15.1 & 13.0 & 10.8 \\
\hline & $15: 40$ & -1.764 & 33.7 & 32.2 & 19.8 & 13.3 & 11.9 & 9.0 & \multirow[t]{10}{*}{82} & $15: 40$ & -1.764 & 32.8 & 31.3 & 22.0 & 15.2 & 13.2 & 10.8 \\
\hline & $16: 10$ & -1.743 & 33.5 & 34.4 & 29.9 & 12.6 & 12.1 & 9.5 & & $16: 10$ & -1.743 & 33.4 & 39.6 & 31.0 & 18.0 & 13.1 & 10.2 \\
\hline & $16: 40$ & -1.722 & 33.6 & 33.2 & 31.9 & 12.9 & 11.4 & 9.3 & & $16: 40$ & -1.722 & 33.6 & 31.6 & 32.2 & 16.0 & 12.8 & 10.8 \\
\hline & $17: 40$ & -1.681 & 34.3 & 33.4 & 33.2 & 14.9 & 10.8 & 9.2 & & $17: 40$ & -1.681 & 33.2 & 31.8 & 32.8 & 23.6 & 13.9 & 10.7 \\
\hline & $18: 40$ & -1.639 & 34.8 & 33.8 & 31.8 & 22.3 & 12.1 & 9.4 & & $18: 40$ & -1.639 & 33.2 & 31.9 & 33.1 & 27.8 & 15.3 & 10.7 \\
\hline \multirow[t]{5}{*}{ 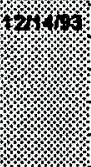 } & $19: 40$ & -4.597 & 34.4 & 32.7 & 32.8 & 28.4 & 12.4 & 9.1 & & $19: 40$ & -1.597 & 33.8 & 32.3 & 33.5 & 29.5 & 19.5 & 10.6 \\
\hline & $20: 40$ & -1.556 & 34.9 & 32.5 & 32.0 & 30.4 & 13.1 & 9.3 & & $20: 40$ & -1.556 & 33.6 & 31.7 & 32.8 & 29.9 & 22.6 & 10.8 \\
\hline & $21: 40$ & -1.514 & 34.2 & 33.3 & 32.3 & 30.8 & 15.4 & 8.1 & & $21: 40$ & -1.514 & 33.6 & 32.2 & 33.8 & 29.8 & 28.8 & 10.5 \\
\hline & $22: 40$ & -1.472 & 34.6 & 32.7 & 32.8 & 31.3 & 18.3 & 9.1 & & $22: 40$ & $-1,472$ & 34.0 & 31.7 & 33.3 & 30.1 & 27.8 & 11.7 \\
\hline & $23: 40$ & -1.431 & 34.2 & 33.4 & 32.3 & 31.7 & 23.3 & 9.0 & & 23:40 & -1.431 & 33.5 & 31.9 & 33.4 & 30.3 & 28.2 & 13.0 \\
\hline \multirow[t]{9}{*}{ 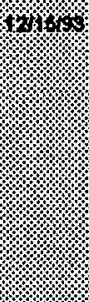 } & $0: 40$ & -1.389 & 34.0 & 33.7 & 32.7 & 31.6 & 26.0 & 9.2 & \multirow[t]{19}{*}{57} & $0: 40$ & -4.369 & 33.5 & 31.4 & 34.0 & 30.4 & 28.2 & 18.0 \\
\hline & $1: 40$ & -1.347 & 34.4 & 32.6 & 32.8 & 30.8 & 27.3 & 9.4 & & $1: 40$ & -1.347 & 34.1 & 32.2 & 33.3 & 30.2 & 28.2 & 21.6 \\
\hline & $2: 40$ & -1.306 & 33.8 & 33.4 & 32.5 & 31.4 & 27.3 & 9.8 & & $2: 40$ & $-1,306$ & 33.7 & 32.0 & 33.6 & 30.6 & 28.1 & 25.1 \\
\hline & $3: 40$ & -1.264 & 33.7 & 33.5 & 32.4 & 31.7 & 28.3 & 11.1 & & $3: 40$ & -1.264 & 34.3 & 32.1 & 33.4 & 30.0 & 27.8 & 28.4 \\
\hline & $4: 40$ & -1.222 & 33.4 & 33.7 & 32.7 & 32.5 & 28.1 & 12.5 & & $4: 40$ & -1.222 & 33.7 & 32.2 & 32.8 & 30.0 & 28.3 & 26.4 \\
\hline & $5: 40$ & -1.181 & 34.3 & 33.3 & 32.3 & 31.6 & 27.6 & 16.1 & & $5: 40$ & -1.181 & 33.3 & 32.6 & 33.3 & 30.7 & 28.6 & 26.3 \\
\hline & $6: 40$ & -1.139 & 34.4 & 32.9 & 32.8 & 31.6 & 28.3 & 19.8 & & $6: 40$ & -1.139 & 32.8 & 32.4 & 33.2 & 30.8 & 28.1 & 26.2 \\
\hline & $7: 40$ & -1.097 & 34.4 & 33.7 & 32.6 & 31.7 & 27.9 & 23.4 & & $7: 40$ & -1.097 & 33.3 & 31.8 & 33.1 & 30.8 & 27.8 & 27.3 \\
\hline & $8: 40$ & -1.036 & 34.0 & 33.7 & 32.9 & 32.2 & 28.8 & 26.7 & & $8: 40$ & -1.056 & 33.3 & 32.4 & 33.0 & 30.4 & 27.8 & 27.2 \\
\hline \multirow[t]{5}{*}{ 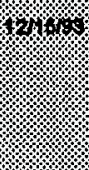 } & $9: 40$ & -1.014 & 33.9 & 33.4 & 32.7 & 32.0 & 28.2 & 26.9 & & $8: 40$ & -1.014 & 33.1 & 31.8 & 33.0 & 31.0 & 27.6 & 27.4 \\
\hline & $10: 40$ & -0.972 & 34,1 & 33.0 & 32.9 & 31.8 & 28.7 & 27.4 & & $10: 40$ & -0.972 & 33.2 & 31.8 & 34.1 & 30.7 & 28.8 & 27.6 \\
\hline & $11: 40$ & -0.931 & 34.3 & 33.6 & 33.0 & 31.8 & 28.3 & 27.6 & & $11: 40$ & -0.931 & 33.3 & 31.9 & 33.8 & 30.1 & 28.2 & 27.5 \\
\hline & $15 ; 40$ & 0.764 & 34.7 & 33.5 & 32.8 & 32.0 & 28.7 & 28.5 & & $15: 40$ & -0.764 & 34.1 & 32.2 & 33.4 & 31.7 & 28.2 & 28.8 \\
\hline & $19: 40$ & -0.597 & 33.8 & 33.4 & 33.0 & 31.7 & 28.6 & 29.3 & & $19: 40$ & -0.597 & 33.7 & 31.6 & 33.8 & 30.6 & 28.4 & 28.2 \\
\hline \multirow[t]{5}{*}{$8 \%$} & $5: 40$ & 0.181 & 35.1 & 33.4 & 333.5 & 32.2 & 29.6 & 29.1 & & $5: 40$ & -0.181 & 33.2 & 32.3 & 33.5 & 31.6 & 29.3 & 28.9 \\
\hline & $8: 40$ & -0.056 & 34.1 & 33.8 & 33.3 & 32.5 & 29.0 & 29.7 & & $8: 40$ & -0.056 & 33.8 & 32.3 & 33.8 & 31.2 & 28.0 & 28.4 \\
\hline & $10: 00$ & 0.000 & 34.7 & 33.6 & 33.5 & 32.5 & 28.9 & 29.5 & & $10: 00$ & 0.000 & 34.1 & 33.0 & 34.7 & 31.3 & 28.2 & 28.6 \\
\hline & $11: 00$ & 0.042 & 34.8 & 33.3 & 33.4 & 32.6 & 29.5 & 29.6 & & $11: 00$ & 0.042 & 33.8 & 32.6 & 34.5 & 30.8 & 29.1 & 28.8 \\
\hline & $12: 00$ & 0.083 & 34.2 & 34.0 & 33.8 & 32.5 & 29.4 & 29.7 & & $12: \infty$ & 0.083 & 34.2 & 32.8 & 34.8 & 31.7 & 28.3 & 29.0 \\
\hline
\end{tabular}


Table 4. Neutron Moisture Meter Data.

\begin{tabular}{|c|c|c|c|c|c|c|c|c|c|c|c|c|c|c|c|c|c|}
\hline \multicolumn{18}{|c|}{ Neutron Molsture Meter Data. } \\
\hline & & & & & & & & & & & & & & & & & \\
\hline & & & \multicolumn{5}{|c|}{ Access Tube, Southeast Comer of IP Site } & & & & & \multicolumn{5}{|c|}{ Access Tube, Center of IP site } & \\
\hline & & & & & & & & & & & & & & & & & \\
\hline & & Test & $\begin{array}{l}\text { Volumetric } \\
\text { Moisture } \\
\text { Content } y\end{array}$ & $\begin{array}{l}\text { Volumetric } \\
\text { Moisture } \\
\text { Content \% }\end{array}$ & \begin{tabular}{|c|} 
Volumetric \\
Molsture \\
Content $\%$
\end{tabular} & $\begin{array}{l}\text { Volumetric } \\
\text { Moisture } \\
\text { Content X }\end{array}$ & $\begin{array}{l}\text { Volumetric } \\
\text { Molsture } \\
\text { Content } \%\end{array}$ & $\begin{array}{l}\text { Volumetric } \\
\text { Molsture } \\
\text { Content } \%\end{array}$ & & & Tost & $\begin{array}{c}\text { Volumetric } \\
\text { Molsture } \\
\text { Content } \%\end{array}$ & $\begin{array}{l}\text { Volumatrle } \\
\text { Molsture } \\
\text { Content } 4\end{array}$ & \begin{tabular}{c|} 
Volumetrle \\
Molsture \\
Content $\%$
\end{tabular} & $\begin{array}{l}\text { Volumetrle } \\
\text { Molsture } \\
\text { Content } \%\end{array}$ & $\begin{array}{l}\text { Volumetric } \\
\text { Molsture } \\
\text { Content } \%\end{array}$ & $\begin{array}{l}\text { Volumatric } \\
\text { Molsture } \\
\text { Content \% }\end{array}$ \\
\hline \multirow[t]{4}{*}{ Date } & Time & Days' & 6. & 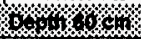 & $5.1 \%$ & 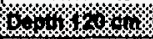 & woros & 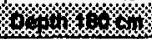 & Date & Time & Days' & 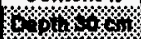 & $6 \%$ & \%力 & w & Y.t. & ow \\
\hline & $13: 00$ & 0.125 & 34.0 & 34.0 & 33.2 & 32.4 & 28.6 & 29.9 & & $13: 00$ & 0.125 & 33.8 & 32.3 & 34.5 & 31.7 & 28.9 & 29.1 \\
\hline & $14: 00$ & 0.167 & 33.6 & 33.6 & 32.7 & 32.1 & 29.3 & 29.6 & & $14: 00$ & 0.167 & 33.5 & 32.2 & 33.7 & 31.3 & 28.5 & 29.0 \\
\hline & $15: 00$ & 0.208 & 33.0 & 33.3 & 33.5 & 32.4 & 29.5 & 28.7 & & $15: 00$ & 0.208 & 32.5 & 32.3 & 34.6 & 31.2 & 29.4 & 28.5 \\
\hline \multirow[t]{8}{*}{1810} & $16: 00$ & 0.250 & 31.8 & 33.5 & 33.0 & 32.3 & 28.7 & 29.1 & & $16: 00$ & 0.250 & 31.4 & 32.0 & 33.8 & 31.2 & 28.9 & 27.8 \\
\hline & $17: 00$ & 0.292 & 30.7 & 33.7 & 32.7 & 32.4 & 29.6 & 28.4 & & $17: 00$ & 0.292 & 30.8 & 32.5 & 34.1 & 31.4 & 28.7 & 28.1 \\
\hline & $18: 00$ & 0.333 & 30.3 & 33.4 & 33.2 & 32.1 & 29.2 & 28.7 & & $18: 00$ & 0.333 & 29.0 & 31.5 & 34.3 & 31.5 & 28.6 & 27.8 \\
\hline & $19: 00$ & 0.375 & 28.8 & 32.7 & 33.1 & 32.1 & 28.8 & 28.1 & & $19: 00$ & 0.375 & 28.6 & 32.0 & 34.5 & 31.0 & 28.4 & 27.6 \\
\hline & $20: 00$ & 0.417 & 28.8 & 32.0 & 32.5 & 32.3 & 28.5 & 28.4 & & $20: 00$ & 0.417 & 28.7 & 31.7 & 34.3 & 31.2 & 28.7 & 27.4 \\
\hline & $21: 00$ & 0.456 & 28.5 & 32.0 & 33.4 & 32.6 & 28.5 & 27.8 & & $21: 00$ & 0.458 & 28.0 & 31.8 & 34.5 & 30.5 & 28.4 & 27.3 \\
\hline & $22: 00$ & 0.500 & 28.3 & 31,4 & 32.9 & 31.7 & 28.6 & 27.8 & & $22: 00$ & 0.500 & 27.1 & 31.4 & 33.8 & 31.5 & 28.2 & 27.5 \\
\hline & $23: 00$ & 0.542 & 27.4 & 31.3 & 32.4 & 32.4 & 28.3 & 28.1 & & $23: 00$ & 0.542 & 26.8 & 31.0 & 34.3 & 31.1 & 28.0 & 27.1 \\
\hline \multirow[t]{20}{*}{ 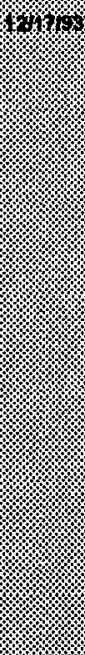 } & 0.00 & 0.383 & 27.1 & 30.8 & 32.1 & 32.3 & 28.5 & 28.5 & \multirow{6}{*}{ \% } & 0.00 & 0.583 & 28.5 & 31.4 & 33.5 & 31.4 & 28.4 & 28.9 \\
\hline & $1: 00$ & 0.625 & 28.1 & 30.2 & 31.4 & 31.4 & 28.5 & 27.6 & & $1: 00$ & 0.625 & 26.6 & 31.3 & 33.5 & 30.8 & 28.2 & 27.4 \\
\hline & $2: 00$ & 0.667 & 26.5 & 30.8 & 31.8 & 31.5 & 28.3 & 27.3 & & $2: 00$ & 0.667 & 25.8 & 31.1 & 33.7 & 31.0 & 27.6 & 26.8 \\
\hline & $3: 00$ & 0.708 & 26.4 & 30.5 & 31.8 & 31.7 & 28.1 & 27.0 & & $3: 00$ & 0.708 & 26.3 & 30.5 & 33.4 & 30.9 & 27.7 & 28.7 \\
\hline & $4: 00$ & 0.750 & 25.7 & 29.4 & 31.9 & 31.7 & 28.7 & 27.0 & & $4: 00$ & 0.750 & 25.8 & 30.0 & 34.1 & 30.6 & 27.8 & 26.8 \\
\hline & 5.00 & 0.792 & 25.6 & 29.4 & 31.4 & 31.6 & 28.4 & 28.8 & & $5: 00$ & 0.792 & 25.4 & 30.1 & 33.7 & 30.8 & 27.9 & 28.8 \\
\hline & 600 & 0.833 & 24.9 & 29.2 & 31.7 & 31.3 & 28.3 & 28.6 & \multirow[t]{13}{*}{ 1 $14 x$} & $6: 00$ & 0.833 & 24.7 & 30.2 & 32.8 & 30.9 & 27.7 & 28.5 \\
\hline & $7: 00$ & 0.875 & 25.4 & 29.6 & 31.9 & 30.8 & 28.5 & 28.6 & & $7: 00$ & 0.875 & 24.6 & 29.9 & 34.0 & 30.9 & 27.7 & 26.4 \\
\hline & $8: 00$ & 0.917 & 24.9 & 28.8 & 31.3 & 31.0 & 27.8 & 27.5 & & $8: 00$ & 0.917 & 24.7 & 30.3 & 33.6 & 31.5 & 27.8 & 28.1 \\
\hline & $9: 00$ & 0.958 & 24.9 & 28.5 & 31.1 & 31.3 & 28.2 & 27.1 & & $8: 00$ & 0.958 & 24.8 & 29.5 & 32.8 & 30.3 & 28.6 & 25.8 \\
\hline & $10: 00$ & 1.000 & 24.9 & 28.0 & 30.9 & 31.2 & 28.7 & 27.2 & & $10: 00$ & 1.000 & 24.7 & 29.4 & 33.9 & 31.3 & 28.3 & 28.2 \\
\hline & $11: 00$ & 1.042 & 24.6 & 28.7 & 31.2 & 31.2 & 28.8 & 28.4 & & $11: 00$ & 1.042 & 24.5 & 29.1 & 33.8 & 30.9 & 27.8 & 25.8 \\
\hline & $12: 00$ & 1.083 & 24.6 & 28.1 & 31.1 & 31.1 & 28.4 & 26.4 & & $12: 00$ & 1.083 & 24.8 & 28.8 & 33.5 & 31.2 & 28.1 & 25.8 \\
\hline & $13: 00$ & 1.125 & 24.6 & 28.6 & 31.4 & 31.5 & 28.1 & 28.5 & & $13: 00$ & 1.125 & 24.2 & 29.3 & 33.2 & 31.0 & 28.1 & 25.8 \\
\hline & $14: 00$ & 1.167 & 24.7 & 27.8 & 31.0 & 31.5 & 28.3 & 28.2 & & $14: 00$ & 3.167 & 24.2 & 28.7 & 32.7 & 31.0 & 27.7 & 28.0 \\
\hline & $15: 00$ & 1.208 & 23.7 & 27.2 & 30.2 & 31.3 & 27.8 & 28.4 & & $15: 00$ & 1.208 & 24.1 & 29.1 & 32.8 & 30.5 & 28.1 & 25.4 \\
\hline & $16: 00$ & 1.250 & 24.6 & 27.8 & 29.9 & 31.3 & 28.3 & 25.7 & & $16: \infty$ & 1.250 & 23.7 & 28.1 & 33.2 & 31.0 & 27.3 & 25.6 \\
\hline & $17: 00$ & 1.292 & 24.0 & 27.4 & 29.8 & 31.8 & 28.5 & 28.0 & & $17: 00$ & 1.292 & 24.4 & 28.0 & 32.5 & 30.8 & 27.9 & 25.8 \\
\hline & $18: 00$ & 1.333 & 24.2 & 27.2 & 30.4 & 31.4 & 28.0 & 28.1 & & $18: 00$ & 1.333 & 23.6 & 28.2 & 33.1 & 30.0 & 27.2 & 23.7 \\
\hline & $22: 00$ & 1.500 & 23.6 & 26.8 & 28.9 & 31.1 & 28.0 & 25.7 & \multirow{7}{*}{ 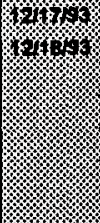 } & $22: 00$ & 1.500 & 23.5 & 27.1 & 32.3 & 30.0 & 27.5 & 24.8 \\
\hline \multirow[t]{6}{*}{$4 \%$} & $2: 00$ & 1.667 & 23.6 & 26.3 & 29.5 & 31.0 & 27.6 & 25.6 & & 200 & 1.667 & 23.0 & 26.7 & 32.2 & 30.5 & 27.4 & 24.4 \\
\hline & $6: 00$ & 1.833 & 23.8 & 26.7 & 29.2 & 31.0 & 27.0 & 25.2 & & $6: 00$ & 1.833 & 23.0 & 26.8 & 32.0 & 30.0 & 27.1 & 24.5 \\
\hline & $70: 00$ & 2.000 & 23.1 & 26.0 & 29.3 & 31.3 & 27.6 & 24.5 & & 10.00 & 2.000 & 23.0 & 28.3 & 31.1 & 30.3 & 28.9 & 24.5 \\
\hline & $14: 00$ & 2.167 & 22.7 & 25.9 & 29.1 & 30.9 & 28.1 & 24.1 & & $14: 00$ & 2.167 & 22.6 & 28.0 & 31.1 & 29.8 & 28.3 & 24.0 \\
\hline & $18: 00$ & 2.333 & 22.8 & 25.0 & 28.8 & 30.8 & 27.6 & 23.8 & & $18: 00$ & 2.333 & 22.8 & 28.0 & 31,7 & 29.9 & 27.1 & 23.8 \\
\hline & $22: 00$ & 2.500 & 22.9 & 25.4 & 28.9 & 30.1 & 26.9 & 23.9 & & $22: 00$ & 2.500 & 22.1 & 25.7 & 30.5 & 29.5 & 26.3 & 24.0 \\
\hline \multirow[t]{2}{*}{ W } & $2: 00$ & 2.667 & 23.1 & 25.6 & 28.1 & 30.6 & 27.3 & 24.4 & \multirow[t]{2}{*}{1119} & $2: 00$ & 2.667 & 22.0 & 25.4 & 30.8 & 29.8 & 28.6 & 23.9 \\
\hline & $6: 00$ & 2.833 & 23.0 & 24.8 & 27.7 & 30.0 & 27.1 & 23.5 & & $6: 00$ & 2.833 & 220 & 25.5 & 30.6 & 29.8 & 28.5 & 23.5 \\
\hline
\end{tabular}


Table 4. Neutron Moisture Meter Data.

\begin{tabular}{|c|c|c|c|c|c|c|c|c|c|c|c|c|c|c|c|c|c|}
\hline \multicolumn{18}{|c|}{ Neutron Molsture Meter Data. } \\
\hline & & & & & & & & & & & & \multirow{2}{*}{\multicolumn{5}{|c|}{ Access Tube, Centor of IP Site }} & \\
\hline & & & \multicolumn{5}{|c|}{ Access Tube, Southeast Corner of IP Site } & & & & & & & & & & \\
\hline & & & & & & & & & & & & & & & & & \\
\hline & & Test & $\begin{array}{l}\text { Volumetric } \\
\text { Moisture } \\
\text { Content } \%\end{array}$ & $\begin{array}{l}\text { Volumetric } \\
\text { Moisture } \\
\text { Content\% }\end{array}$ & $\begin{array}{l}\text { Volumetric } \\
\text { Moisture } \\
\text { Content } x\end{array}$ & $\begin{array}{l}\text { Volumetric } \\
\text { Molsture } \\
\text { Content } \%\end{array}$ & $\begin{array}{l}\text { Volumetric } \\
\text { Molsture } \\
\text { Content } \%\end{array}$ & $\begin{array}{l}\text { Volumetric } \\
\text { Molsture } \\
\text { Content } x\end{array}$ & & & Test & $\begin{array}{c}\text { Volumetric } \\
\text { Molisture } \\
\text { Content } x\end{array}$ & \begin{tabular}{c|} 
Volumetric \\
Moistur \\
Content $x$
\end{tabular} & $\begin{array}{l}\text { Volumetric } \\
\text { Molsture } \\
\text { Conteme }\end{array}$ & $\begin{array}{l}\text { Volumetric } \\
\text { Molisture } \\
\text { Content } \%\end{array}$ & $\begin{array}{l}\text { Volumetric } \\
\text { Molsture } \\
\text { Content } 4\end{array}$ & $\begin{array}{l}\text { Volumetric } \\
\text { Moisture } \\
\text { Content } x\end{array}$ \\
\hline Date & Time & Days' & (ox on & 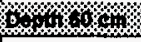 & 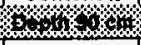 & $6 \%$ w & 16 & 10.6. & Date & Time & Days' & 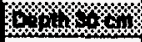 & 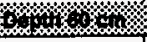 & rom & 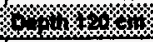 & mon. & 80 \\
\hline & 10:00 & 3.000 & 22.4 & 24.6 & 27.6 & 29.4 & 26.8 & 23.6 & & $10: 00$ & 3.000 & 22.1 & 24.4 & 30.7 & 30.1 & 28.0 & 23.4 \\
\hline & $14: 00$ & 3.167 & 22.9 & 24.7 & 28.0 & 30.8 & 26.8 & 23.1 & & $14: 00$ & 3.167 & 21.6 & 24.6 & 30.5 & 29.9 & 28.0 & 23.5 \\
\hline 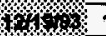 & $18: 00$ & 3.333 & 22.4 & 24.6 & 27.6 & 30.8 & 28.8 & 23.3 & 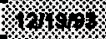 & $18: 00$ & 3.333 & 21.4 & 24.7 & 30.1 & 29.3 & 25.6 & 23.5 \\
\hline (x) & 6:00 & 3.833 & 22.9 & 24.8 & 27.3 & 30.4 & 28.7 & 22.8 & $1 \%$ & $8: 00$ & 3.833 & 22.1 & 24.5 & 30.0 & 28.7 & 25.4 & 23.1 \\
\hline & $14: 00$ & 4.167 & 22.4 & 23.8 & 27.1 & 30.9 & 26.1 & 22.4 & & $14: 00$ & 4.167 & 21.3 & 24.0 & 29.9 & 29.0 & 25.1 & 22.8 \\
\hline & $22: 00$ & 4.500 & 22.1 & 24.3 & 27.0 & 30.4 & 28.2 & 22.9 & & $22: 00$ & 4.500 & 21.0 & 23.8 & 30.1 & 28.7 & 25.2 & 22.8 \\
\hline \% & $6: 00$ & 4.833 & 22.0 & 23.8 & 28.8 & 30.1 & 28.5 & 22.7 & 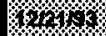 & $6: 00$ & 4.833 & 21.1 & 23.8 & 30.0 & 29.3 & 24.8 & 22.4 \\
\hline & 18:00 & 5.333 & 21.8 & 23.7 & 26.3 & 29.9 & 28.6 & 22.0 & & $18: 00$ & 5.333 & 20.3 & 23.3 & 28.9 & 28.8 & 24.4 & 22.2 \\
\hline 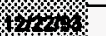 & $8: 00$ & 5.917 & 21.5 & 23.1 & 28.3 & 29.2 & 26.3 & 21.7 & $12 \% 1$ & 8.00 & 8.917 & 20.4 & 22.9 & 29.1 & 28.0 & 24.7 & 22.1 \\
\hline & $20: 00$ & 6.417 & 21.7 & 23.1 & 26.3 & 29.8 & 28.1 & 22.1 & & $20: 00$ & 6.417 & 20.4 & 23.4 & 28.8 & 28.8 & 24.8 & 21.6 \\
\hline 1024 & $8: 30$ & 6.938 & 21.4 & 23.2 & 25.5 & 29.7 & 25.7 & 21.7 & W. & $8: 30$ & 6.938 & 20.4 & 22.7 & 28.6 & 28.8 & 25.0 & 21.7 \\
\hline (6.24) & $10: 00$ & 8.000 & 21.2 & 23.0 & 25.5 & 29.0 & 28.5 & 21.1 & 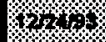 & $10: 00$ & 8.000 & 19.8 & 22.5 & 28.2 & 28.6 & 24.1 & 21.1 \\
\hline 1228,6 & $10: 00$ & 9.000 & 20.5 & 22.2 & 25.0 & 29.5 & 25.5 & 20.4 & $\%$ & $10: 00$ & 9.000 & 19.8 & 22.9 & 28.6 & 28.0 & 24.7 & 20.8 \\
\hline 12. 6 - & $10: 00$ & 10.000 & 20.3 & 22.5 & 24.8 & 29.1 & 28.1 & 21.2 & 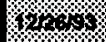 & $10: 00$ & 10.000 & 19.5 & 21.8 & 27.7 & 28.0 & 24.7 & 21.5 \\
\hline $1771 \times$ & $10: 00$ & 11.000 & 20.8 & 22.2 & 24.3 & 29.0 & 25.7 & 20.6 & $1 \% 114$ & $10: 00$ & 11.000 & 20.1 & 21.1 & 27.4 & 27.9 & 24.1 & 20.9 \\
\hline $12,8,1$ & $10: 00$ & 12.00 & 20.1 & 21.8 & 23.7 & 28.7 & 25.2 & 20.4 & $12 \times 1.4$ & $10: 00$ & 12.000 & 19.6 & 21.2 & 27.2 & 27.4 & 23.7 & 20.9 \\
\hline $14-4=$ & $10: 00$ & 13.00 & 20.0 & 21.8 & 23.7 & 28.7 & 25.2 & 19.8 & $10 \%, 4$ & $10: 00$ & 13.000 & 19.0 & 21.6 & 27.5 & 27.8 & 23.8 & 20.5 \\
\hline $122 \%$ & $10: 00$ & 17.00 & 19.7 & 21.2 & 22.8 & 28.8 & 24.4 & 19.5 & 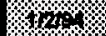 & $10: 00$ & 17.000 & 18.1 & 20.9 & 28.0 & 27.2 & 23.7 & 19.7 \\
\hline 1818 & $10: 00$ & 18.00 & 19.3 & 21.2 & 23.0 & 28.2 & 24.8 & 19.4 & 194 & $10: 00$ & \begin{tabular}{|l}
18.000 \\
\end{tabular} & 18.7 & 20.8 & 27.1 & 28.9 & 23.0 & 20.2 \\
\hline 14. & $10: 00$ & 19.00 & 19.4 & 21.1 & 22.9 & 28.4 & 24.7 & 19.2 & $\%$ & $10: 00$ & 19.000 & 18.5 & 20.7 & 26.3 & 26.6 & 23.4 & 19.5 \\
\hline$\sqrt{1} \%$ & $10: 20$ & 20.01 & 19.4 & 21.1 & 22.6 & 27.7 & 24.0 & 19.2 & 113 & $10: 20$ & 20.014 & 18.5 & 20.7 & 28.4 & 26.8 & 23.3 & 19.8 \\
\hline$\sqrt{2} 1 \%$ & 9:50 & 25.99 & 19.1 & 20.2 & 21.8 & 27.8 & 23.7 & 19.2 & \%x & $8: 40$ & \begin{tabular}{|l}
25.986 \\
\end{tabular} & 17.8 & 19.9 & 26.1 & 26.5 & 22.1 & 18.3 \\
\hline \% & $10: 20$ & 34.01 & 18.6 & 20.4 & 21.4 & 27.1 & 23.4 & 18.4 & $111 \%$ & $10: 20$ & \begin{tabular}{|l|}
34.014 \\
\end{tabular} & 17.5 & 19.6 & 25.7 & 28.1 & 21.6 & 19.3 \\
\hline 1404 & $1: 20$ & 41.14 & 18.7 & 19.7 & 20.9 & 28.1 & 23.8 & 17.8 & 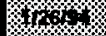 & 1:25 & 41.142 & $\$ 7.2$ & 18.1 & 24.8 & 26.2 & 22.4 & 18.6 \\
\hline \% & 11:00 & 48.19 & 48.2 & 19.8 & 20.6 & 28.1 & 23.2 & 17.7 & 梦梦 & $2: 30$ & 48.188 & 17.2 & 18.2 & 24.2 & 25.8 & 21.0 & 18.8 \\
\hline 24 & $11: 00$ & 55.04 & 19.8 & 20.0 & 20.5 & 26.1 & 22.9 & 18.3 & (24. & $11: 00$ & 55.042 & 17.5 & 19.0 & 24.4 & 26.3 & 21.4 & 18.2 \\
\hline $4 \%$ & $11: 00$ & 62.04 & 18.7 & 19.8 & 19.7 & 28.6 & 22.5 & 17.4 & $17 \%$ & $11: 00$ & 62.042 & 17.7 & 18.8 & 24.5 & 25.2 & 21.1 & 18.2 \\
\hline 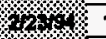 & 11:00 & 69.04 & 18.3 & 19.1 & 19.7 & 25.2 & 21.9 & 17.8 & $2 \%$ & $11: 00$ & 69.043 & 16.8 & 18.5 & 23.4 & 25.3 & 21.1 & 17.8 \\
\hline . & 11:00 & 76.04 & 18.2 & 19.5 & 19.7 & 25.6 & 22.1 & 17.3 & $\%$ & $11: 00$ & 76.043 & 16.8 & 18.0 & 23.7 & 25.4 & 21.2 & 18.2 \\
\hline , & 11:00 & 83.04 & 18.9 & 19.4 & 19.4 & 25.6 & 22.0 & 16.8 & 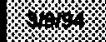 & $11: 00$ & 83.042 & 17.8 & \begin{tabular}{ll|}
18.1 \\
\end{tabular} & 23.5 & 26.1 & 20.8 & 18.5 \\
\hline 7. & $11: 00$ & 90.04 & 19.3 & 19.5 & 19.6 & 24.9 & 22.6 & 16.9 & $40 \%$ & $11: 00$ & 90.043 & 17.8 & 18.4 & 23.2 & 24.8 & 20.6 & 17.8 \\
\hline $4 \%$ & $11: 00$ & 97.04 & 19.2 & 18.8 & 18.1 & 25.8 & 21.8 & 16.3 & W & $11: 00$ & 97.043 & 17.4 & 18.1 & 22.8 & 25.2 & 20.8 & 17.7 \\
\hline 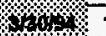 & $11: 00$ & 104.13 & 18.5 & 18.7 & 18.8 & 25.0 & 22.1 & 16.5 & $3 / 10 \%$ & $13: 00$ & 104.133 & 17.1 & 18.0 & 22.9 & 25.2 & 20.3 & 17.4 \\
\hline \% & 11:30 & 111.18 & 48.0 & 18.8 & 19.0 & 25.7 & 21.2 & 16.8 & \% & $11: 30$ & 111.183 & 17.3 & 17.8 & 22.3 & 25.7 & 20.8 & 17.5 \\
\hline 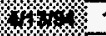 & $11: 00$ & 118.04 & 17.8 & 18.6 & 18.7 & 25.1 & 21.4 & 16.8 & 214 & $11: 00$ & 118.043 & 16.8 & 17.4 & 22.5 & 25.0 & 18.7 & 18.1 \\
\hline$\gamma_{7}$ & $14: 30$ & 128.19 & 17.7 & 18.5 & 18.8 & 25.7 & 21.5 & 18.7 & $8 \%$ & $14: 30$ & 125.194 & 16.8 & 17.9 & 22.8 & 24.8 & 20.5 & 17.5 \\
\hline $18 \% 1 \%$ & 11:30 & 132.19 & 17.2 & 18.7 & 18.8 & 25.3 & 21.0 & 17.0 & 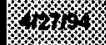 & $11: 30$ & 132.193 & 16.4 & 17.4 & 22.0 & 24.7 & 20.1 & 17.8 \\
\hline 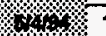 & $13: 00$ & 139.08 & 17.5 & 18.7 & 18.1 & 24.5 & 21.6 & 18.2 & $8 \%$ & $13: 00$ & 139.113 & 16.4 & 17.0 & 22.3 & 25.0 & 19.9 & 17.6 \\
\hline \% & $13: 10$ & 147.08 & 17.4 & 18.1 & 18.1 & 24.9 & 21.7 & 16.6 & 6 & $13: 00$ & 147.113 & 15.8 & 17.4 & 22.0 & 24.8 & 19.7 & 17.2 \\
\hline
\end{tabular}


Table 4. Neutron Moisture Meter Data.

\begin{tabular}{|c|c|c|c|c|c|c|c|c|c|c|c|c|c|c|c|c|c|}
\hline \multicolumn{18}{|c|}{ Neutron Moisture Meter Data. } \\
\hline & & & & & & & & & & & & & & & & & \\
\hline & & & \multicolumn{5}{|c|}{ Access Tube, Southeast Comer of IP site } & & & & & \multicolumn{5}{|c|}{ Access Tube, Center of IP Site } & \\
\hline & & & & & & & & & & & & & & & & & \\
\hline & & Test & $\begin{array}{l}\text { Volumetric } \\
\text { Molsture } \\
\text { Content \% }\end{array}$ & $\begin{array}{l}\text { Volumetric } \\
\text { Molsture } \\
\text { Content\% }\end{array}$ & \begin{tabular}{|l|} 
Volumetric \\
Molsture \\
Content $\%$
\end{tabular} & $\begin{array}{l}\text { Volumetric } \\
\text { Molsture } \\
\text { Content } \%\end{array}$ & $\begin{array}{l}\text { Volumetric } \\
\text { Moisture } \\
\text { Content } 4\end{array}$ & $\begin{array}{l}\text { Volumetric } \\
\text { Molsture } \\
\text { Content } \%\end{array}$ & & & Test & $\begin{array}{l}\text { Volumetrle } \\
\text { Molsture } \\
\text { Content } \%\end{array}$ & $\begin{array}{l}\text { Volumetric } \\
\text { Molsture } \\
\text { Contunt } \%\end{array}$ & $\begin{array}{l}\text { Volumetrle } \\
\text { Molsture } \\
\text { Contunt } \%\end{array}$ & $\begin{array}{l}\text { Volumetric } \\
\text { Molsture } \\
\text { Content } 4\end{array}$ & $\begin{array}{l}\text { Volumetric } \\
\text { Moisture } \\
\text { Contenty }\end{array}$ & $\begin{array}{l}\text { Volumetric } \\
\text { Molsture } \\
\text { Content } \\
\end{array}$ \\
\hline Date & Time & Days ${ }^{1}$ & now & 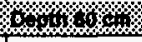 & 00 & $00 \alpha_{1}$ & 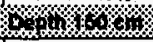 & 80 & Date & Time & Days' & $0.1 \%$ & onton & ox & x & 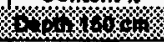 & - \\
\hline 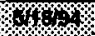 & $11: \infty$ & 153.08 & 17.4 & 18.1 & 18.1 & 24.7 & 21.0 & 18.7 & $847 \%$ & $11: 00$ & 153.013 & 16.3 & 17.2 & 22.4 & 24.4 & 19.5 & 16.7 \\
\hline $44 \%$ & $14: 40$ & 159.08 & 17.6 & 18.3 & 18.3 & 24.9 & 21.5 & 17.2 & 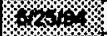 & $14: 30$ & 160.203 & 16.0 & 16.8 & 22.8 & 24.7 & 20.4 & 17.4 \\
\hline 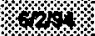 & $11: 00$ & 168.08 & 18.4 & 18.3 & 17.8 & 24.9 & 20.8 & 18.3 & $1 \%$ & $11: 00$ & 168.013 & 15.9 & 17.0 & 22.0 & 24.7 & 18.8 & 17.4 \\
\hline $48 \%$ & $12: 40$ & 174.11 & 18.3 & 17.7 & 18.4 & 24.9 & 21.0 & 18.5 & 16 & $12: 30$ & 174.113 & 16.4 & 16.9 & 21.8 & 24.1 & 19.4 & 17.6 \\
\hline 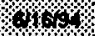 & $13: 00$ & 182.12 & 18.1 & 18.6 & 18.0 & 25.3 & 21.2 & 18.2 & $816 \% 8$ & 12:30 & 182.113 & 18.2 & 17.5 & 22.3 & 24.9 & 19.8 & 17.2 \\
\hline $60 \% 1$ & $12: 20$ & 188.11 & 20.1 & 18.5 & 17.8 & 25.0 & 21.0 & 15.8 & 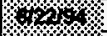 & $12: 00$ & 188.083 & 15.9 & 17.5 & 21.7 & 23.9 & 20.6 & 18.8 \\
\hline 64 & $13: 00$ & 195.12 & 18.1 & 18.8 & 17.7 & 24.3 & 20.6 & 16.2 & 1\% 14 & $13: 00$ & 195.083 & 15.7 & 16.8 & 22.4 & 24.1 & 19.7 & 16.8 \\
\hline 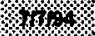 & $9: 00$ & 203.01 & 17.8 & 18.2 & 17.8 & 24.5 & 21.3 & 18.5 & א. & $9: 00$ & 203.013 & 15.9 & 17.0 & 21.8 & 25.2 & 20.0 & 17.0 \\
\hline$\% 1 \% 1 \%$ & $10: 00$ & 209.01 & 17.5 & 18.1 & 17.5 & 24.4 & 20.8 & 15.9 & W $40 \%$ & $10: 00$ & 209.013 & 15.2 & 16.6 & 22.2 & 24.1 & 18.9 & 16.9 \\
\hline $100 \%$ & $11: 30$ & 216.01 & 17.6 & 17.8 & 17.6 & 24.1 & 21.6 & 18.1 & 1100 & $11: 30$ & 216.013 & 15.2 & 16.9 & 21.8 & 24.8 & 20.2 & 17.6 \\
\hline $1 \times 1 \%$ & $14: 30$ & 225.21 & 17.8 & 17.8 & 17.8 & 24.4 & 20.8 & 15.8 & $5 \%$ w & 14:30 & 225.213 & 15.4 & 16.2 & 22.3 & 24.7 & 19.8 & 17.1 \\
\hline $1 \%$ & $15: 03$ & 230.31 & 21.2 & 21.7 & 18.5 & 24.3 & 21.0 & 18.2 & \% & $14: 55$ & 230.213 & 15.1 & 16.9 & 21.6 & 24.8 & 20.1 & 17.3 \\
\hline$\% \% \%$ & $14: 54$ & 237.22 & 18.2 & 19.3 & 18.0 & 24.7 & 20.1 & 16.7 & $6 \%$ H & $14: 45$ & 237.223 & 13.8 & 16.6 & 21.4 & 24.4 & 20.1 & 17.7 \\
\hline $6171 \%$ & $13: 00$ & 244.11 & 22.2 & 23.6 & 23.8 & 27.0 & 21.3 & 18.3 & 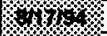 & $13: 00$ & 244.113 & 14.3 & 16.8 & 21.3 & 24.1 & 18.2 & 17.1 \\
\hline 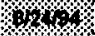 & $12: 30$ & 251.10 & 20.6 & 20.8 & 21.7 & 26.0 & 22.9 & 17.0 & $1 / 4$ & $12: 30$ & 281.104 & 15.4 & 16.7 & 21.5 & 24.5 & 19.7 & 18.8 \\
\hline 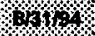 & $11: 30$ & 258.06 & 20.6 & 21.7 & 21.7 & 26.1 & 22.4 & 18.8 & $1131 \%$ & $11: 30$ & 258.063 & 14.8 & 18.2 & 21.2 & 24.5 & 19.3 & 17.3 \\
\hline $4 \% 104$ & $14: 25$ & 274.18 & 19.9 & 19.4 & 20.4 & 25.9 & 20.8 & 17.1 & 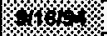 & $14: 32$ & 274.189 & 13.7 & 15.6 & 21.3 & 23.8 & 19.0 & 17.3 \\
\hline 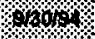 & 12:10 & 288.09 & 18.8 & 19.2 & 18.7 & 25.2 & 21.3 & 16.3 & W & $12: 00$ & 288.083 & 14.5 & 16.1 & 20.4 & 23.4 & 19.2 & 16.7 \\
\hline $1 \% \%$ & $9: 30$ & 304.98 & 17.8 & 18.2 & 18.4 & 24.4 & 20.8 & 15.9 & $60074 \times$ & $8: 30$ & 304.979 & 13.2 & 16.3 & 19.8 & 23.6 & 18.6 & 17.1 \\
\hline \multirow[t]{2}{*}{ 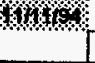 } & $9: 45$ & 329.99 & 17.3 & 18.0 & 16.8 & 23.8 & 19.7 & 18.3 & 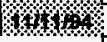 & $9: 30$ & 329.979 & 13.6 & 15.1 & 18.8 & 23.9 & 18.7 & 18.5 \\
\hline & $1=$ & Jays for & which data & was taken. & Includes da & ays prior to $f$ & oding the If & test site. & & & & & & & & & \\
\hline
\end{tabular}


Table 5. Tensiometer Data

\begin{tabular}{|c|c|c|c|c|c|c|c|c|c|c|c|c|c|c|c|c|c|c|c|c|}
\hline & & & & & & & & & Tens: & siometer & Data & & & & & & & & & \\
\hline & & Test & 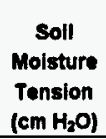 & $\begin{array}{c}\text { Solf } \\
\text { Molsture } \\
\text { Tension } \\
\left(\mathrm{Cm} \mathrm{H}_{2} \mathrm{O}\right) \\
\end{array}$ & 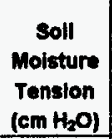 & $\begin{array}{c}\text { Soll } \\
\text { Moisture } \\
\text { Tension } \\
\left(\mathrm{Cm} \mathrm{H}_{2} \mathrm{O}\right) \\
\end{array}$ & 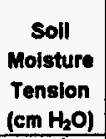 & $\begin{array}{c}\text { Soll } \\
\text { Molsture } \\
\text { Tension } \\
\left(\mathrm{Cm} \mathrm{H}_{2} \mathrm{O}\right) \\
\end{array}$ & $\begin{array}{c}\text { Solf } \\
\text { Moisture } \\
\text { Tension } \\
\left(\mathrm{Cm} \mathrm{H}_{2} \mathrm{O}\right) \\
\end{array}$ & $\begin{array}{c}\text { Soll } \\
\text { Molsture } \\
\text { Tension } \\
\left(\mathrm{Cm} \mathrm{H}_{2} \mathrm{O}\right) \\
\end{array}$ & $\begin{array}{c}\text { Soll } \\
\text { Moisture } \\
\text { Tension } \\
\left(\mathrm{Cm} \mathrm{H}_{2} \mathrm{O}\right)\end{array}$ & $\begin{array}{c}\text { Soll } \\
\text { Moisture } \\
\text { Tension } \\
\left(\mathrm{Cm} \mathrm{H}_{2} \mathrm{O}\right)\end{array}$ & $\begin{array}{c}\text { soll } \\
\text { Molsture } \\
\text { Tenslon } \\
\left(\mathrm{Cm} \mathrm{H}_{2} \mathrm{O}\right)\end{array}$ & $\begin{array}{c}\text { Soll } \\
\text { Molsture } \\
\text { Tonsion } \\
\left.\text { (cm } \mathrm{H}_{2} \mathrm{O}\right) \\
\end{array}$ & $\begin{array}{c}\text { Soll } \\
\text { Molsture } \\
\text { Tonsion } \\
\left(\mathrm{cm} \mathrm{H}_{2} \mathrm{O}\right)\end{array}$ & $\begin{array}{c}\text { Sofl } \\
\text { Molsture } \\
\text { Tension } \\
\left.\text { (cm } \mathrm{H}_{2} \mathrm{O}\right) \\
\end{array}$ & $\begin{array}{c}\text { Soll } \\
\text { Molsture } \\
\text { Tonslon } \\
\left(\mathrm{em} \mathrm{H}_{2} \mathrm{O}\right) \\
\end{array}$ & $\begin{array}{c}\text { Soll } \\
\text { Molsture } \\
\text { Tension } \\
\left(\mathrm{cm}_{2} \mathrm{H}_{3} \mathrm{O}\right) \\
\end{array}$ & $\begin{array}{c}\text { Soll } \\
\text { Molsture } \\
\text { Tension } \\
\left(\mathrm{Cm} \mathrm{H}_{2} \mathrm{O}\right) \\
\end{array}$ & $\begin{array}{c}\text { Soll } \\
\text { Molsture } \\
\text { Tension } \\
\left(\mathrm{Cm} \mathrm{H}_{2} \mathrm{O}\right) \\
\end{array}$ \\
\hline Date & Time & Days' & $C 1^{2}$ & $B 1^{\prime}$ & $\mathbf{A 1}^{\mathbf{2}}$ & $\mathbf{C 2}^{\mathbf{b}}$ & $B 2^{b}$ & $A 2^{b}$ & $\mathrm{C3}^{\mathrm{C}}$ & $\mathbf{B 3}^{\mathrm{c}}$ & $\mathrm{A}^{\mathrm{C}}$ & $\mathrm{CA}^{\mathrm{d}}$ & $B 4^{d}$ & $\mathbf{A A}^{\mathrm{a}}$ & $C 5^{\circ}$ & $B 5^{\circ}$ & $A 5^{\circ}$ & $c 6^{f}$ & $B 6^{\prime}$ & $A 6^{\prime}$ \\
\hline \& & 10:10 & -1.993 & 173.9 & 200.1 & 151.3 & 246.0 & 207.8 & 285.4 & 304.6 & 358.9 & 154.2 & 80.8 & 148.6 & 447.2 & 139.2 & 250.9 & 316.9 & 247.6 & 20.2 & 00.92 \\
\hline 84,40 & $10: 20$ & -1.086 & 168.5 & 199.3 & 156.5 & 251.2 & 221.6 & 280.2 & 301.1 & 358.9 & 155.9 & 80.8 & 159.9 & 448.0 & 140.9 & 255.2 & 318.6 & 250.2 & 91.1 & 81.72 \\
\hline $121 \% 10$ & $10: 30$ & -1.979 & 162.6 & 100.6 & 156.5 & 239.8 & 228.4 & 270.6 & 287.2 & 353.6 & 150.7 & 69.4 & 160.7 & 448.0 & 131.3 & 254.3 & 315.1 & 245.8 & 90.2 & 92.62 \\
\hline 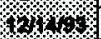 & $10: 40$ & -1.972 & 162.6 & 188.0 & 181.7 & 232.9 & 227.5 & 287.9 & 277.7 & 350.9 & 147.2 & 65.8 & 147.7 & 448.0 & 121.8 & 248.2 & 314.2 & 244.0 & 89.3 & 93.42 \\
\hline $821 \% 1$ & 10:50 & -1.086 & 187.8 & 194.0 & 188.8 & 229.4 & 221.5 & 278.4 & 279.4 & 357.1 & 151.5 & 72.0 & 139.9 & 448.8 & 118.3 & 245.6 & 317.6 & 243.1 & 90.1 & 03.42 \\
\hline $87,1 \%$ & $11: 00$ & -1.058 & 171.2 & 194.9 & 173.9 & 236.4 & 219.7 & 287.1 & 286.3 & 368.5 & 164.5 & 85.1 & 142.4 & 449.7 & 120.0 & 246.4 & 325.3 & 250.8 & 85.3 & 86.82 \\
\hline 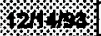 & 11:10 & -1.051 & 169.5 & 187.4 & 184.3 & 250.2 & 212.0 & 287.1 & 281.4 & 378.4 & 178.3 & 83.4 & 139.8 & 450.5 & 118.3 & 250.7 & 332.2 & 255.3 & 98.8 & 88.82 \\
\hline $8 \% \%$ & $11: 20$ & -1.044 & 168.8 & 200.5 & 193.0 & 259.7 & 209.4 & 279.2 & 306.1 & 380.8 & 187.9 & 75.5 & 139.8 & 453.1 & $\begin{array}{l}117.4 \\
\end{array}$ & 251.5 & 339.1 & 255.2 & 99.6 & 97.62 \\
\hline 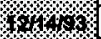 & $11: 30$ & -1.937 & 171.2 & 219.0 & 183.0 & 250.2 & 212.8 & 269.6 & 315.5 & 376.4 & 187.0 & 87.6 & 139.8 & 456.8 & 118.2 & 250.6 & 342.5 & 251.7 & 97.9 & 97.62 \\
\hline 14.4 .9 & $11: 40$ & -1.031 & 185.0 & 223.3 & 191.2 & 244.0 & 214.5 & 267.8 & 315.5 & 373.7 & 187.0 & 87.8 & 143.2 & 457.4 & 120.8 & 254.1 & 344.1 & 254.2 & 94.3 & 97.02 \\
\hline 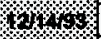 & $11: 50$ & -1.924 & 153.8 & 226.8 & 191.2 & 241.4 & 216.2 & 262.6 & $3+2.0$ & 366.6 & 187.9 & 66.7 & 150.1 & 456.5 & 125.1 & 255.8 & 344.8 & 254.2 & 95.2 & 98.42 \\
\hline $4 \% \%$ & $12: 00$ & -1.917 & 33.2 & 227.6 & 1920 & 237.8 & 217.8 & 262.6 & 307.6 & 373.6 & 187.0 & 63.2 & 171.7 & 455.6 & 125.1 & 265.3 & 344.8 & 255.8 & 85.1 & 88.32 \\
\hline 824,1 & $12: 10$ & -1.910 & 24.6 & 228.7 & 194.8 & 236.1 & 223.0 & 274.7 & 309.3 & 368.6 & 187.0 & 65.0 & 180.4 & 455.8 & 128.8 & 269.6 & 344.8 & 255.8 & 93.4 & 97.52 \\
\hline $827 \% 14$ & $12: 20$ & -1.003 & 17.6 & 227.8 & 195.5 & 243.9 & 231.6 & 282.6 & 317.1 & 368.5 & 187.0 & 65.8 & 182.8 & 455.5 & 128.6 & 273.9 & 345.6 & 258.4 & 96.8 & 87.42 \\
\hline 82.48 & $12: 30$ & -1.896 & 15.0 & 200.7 & 188.7 & 243.8 & 231.5 & 285.2 & 317.0 & 363.9 & 185.2 & 65.8 & 178.0 & 454.7 & 129.4 & 275.6 & 344.8 & 259.3 & 94.2 & 86.62 \\
\hline $854=8$ & $12: 40$ & -1.889 & 12.4 & 56.2 & 87.5 & 247.4 & 235.0 & 284.3 & 323.9 & 368.5 & 185.2 & 63.2 & 180.3 & 451.1 & 132.0 & 273.9 & 345.6 & 259.3 & 82.5 & 84.82 \\
\hline 8 (2) $1 \times$ & $12: 50$ & -1.882 & 9.8 & 32.0 & 28.3 & 241.3 & 235.0 & 273.8 & 322.2 & 368.3 & 185.2 & 59.7 & 179.4 & 446.7 & 131.1 & 267.8 & 344.7 & 256.6 & 24.2 & 83.12 \\
\hline $8121 \% 8$ & $13: 00$ & -1.875 & 9.8 & 18.9 & 15.2 & 237.8 & 240.1 & 269.4 & 320.5 & 368.5 & 181.7 & 59.7 & 182.8 & 444.1 & 128.8 & 267.8 & 345.6 & 258.6 & 93.3 & 81.42 \\
\hline 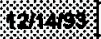 & $13: 10$ & $\begin{array}{l}-1.868 \\
\end{array}$ & 9.8 & 14.7 & 10.8 & 239.5 & 242.7 & 261.6 & 318.7 & 367.4 & 180.0 & 59.7 & 188.3 & 440.8 & & 269.5 & 342.8 & 280.8 & 88.1 & 87.12 \\
\hline $8421 \%$. & $13: 20$ & -1.861 & 9.8 & 9.5 & 7.4 & 237.7 & 249.5 & 266.8 & 322.1 & 366.4 & 181.7 & 60.5 & 193.2 & 444.0 & & 274.6 & 344.6 & 263.5 & 89.8 & 87.02 \\
\hline $4221 \% 4$ & 13:30 & -1.854 & 6.3 & 8.6 & 5.7 & 24.5 & 250.4 & 272.0 & 328.2 & 364.7 & 180.0 & 59.7 & 195.8 & 444.8 & & 275.5 & 344.6 & 263.5 & 89.8 & 86.22 \\
\hline $821 \% 14$ & $13: 40$ & -1.847 & 3.7 & 6.0 & 4.8 & -0.6 & 246.1 & 275.5 & 332.5 & 363.8 & 180.0 & 58.8 & 191.5 & 447.5 & & 273.8 & 343.8 & 280.8 & 89.8 & 86.22 \\
\hline $851.48 \%$ & 13:50 & -1.840 & 2.9 & 6.0 & 4.8 & -6.7 & 251.3 & 275.5 & 337.7 & 362.8 & 178.2 & 56.2 & 195.0 & 447.5 & & 278.4 & 343.8 & 280.8 & 89.8 & 84.52 \\
\hline 812141 & 14:00 & -1.833 & 0.3 & 5.2 & 4.8 & -11.0 & 238.4 & 274.7 & 342.0 & 361.2 & 177.4 & 52.7 & 193.3 & 448.4 & & 278.2 & 343.8 & 281.8 & 88.8 & 83.62 \\
\hline $814 \%$ & 14:10 & -1.826 & -1.5 & 5.2 & 3.8 & -12.8 & 157.5 & 278.4 & 345.5 & 359.4 & 174.8 & 49.2 & 192.4 & 449.3 & & 278.2 & 343.8 & 281.8 & 88.3 & 82.82 \\
\hline $121,4,3$ & 14:20 & -1.819 & -2.3 & 4.3 & 5.7 & .15 .4 & 16.4 & 280.8 & 348.1 & 357.6 & 174.8 & 49.2 & 196.7 & 452.0 & & 279.9 & 343.8 & 281.8 & 86.3 & 82.82 \\
\hline $80,1 \%$ & $14: 30$ & -1.812 & -4.1 & 4.3 & 5.7 & -17.1 & 7.0 & 279.8 & 342.1 & 356.8 & 174.8 & 49.2 & 196.7 & 454.8 & & 279.9 & 343.8 & 203.6 & 88.3 & 81.92 \\
\hline 8123\% & 14:40 & -1.806 & -4.9 & 3.4 & 4.8 & .18 .8 & 4.4 & 178.8 & 198.0 & 354.1 & 171.3 & 51.0 & 191.5 & 454.6 & & 278.9 & 343.0 & 203.6 & 83.7 & 78.52 \\
\hline 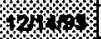 & $14: 50$ & -1.789 & -6.7 & 5.2 & 4.8 & .21 .4 & 0.9 & 12.3 & 18.8 & 352.4 & 171.3 & 51.0 & 189.8 & 452.8 & & 278.1 & 342.1 & 264.5 & 82.9 & 77.62 \\
\hline $825 \%$ & $15: 00$ & -1.782 & .7 .5 & 4.3 & 7.4 & .23 .2 & .6 .0 & 7.1 & 8.3 & 349.7 & 174.8 & 49.2 & 194.1 & 453.7 & & 277.3 & 341.2 & 264.5 & 81.1 & 77.62 \\
\hline $84 \%$ & 15:10 & -1.785 & -8.4 & 3.4 & 8.5 & .24 .0 & .9 .4 & 6.2 & 7.5 & 346.2 & 474.8 & 51.0 & 196.7 & 454.6 & & 278.5 & 342.1 & 286.2 & 79.4 & 75.82 \\
\hline 848.42 & $15: 20$ & -1.778 & .10 .1 & 2.6 & 4.8 & -26.6 & .12 .8 & 2.8 & 4.9 & 345.3 & 178.5 & 50.1 & 198.5 & 455.5 & & 278.2 & 342.1 & 207.1 & 78.5 & 75.02 \\
\hline \% & \begin{tabular}{|l|}
$15: 30$ \\
\end{tabular} & -1.771 & -10.1 & 2.6 & 3.9 & -28.8 & .15 .4 & -0.7 & -4.6 & 345.3 & 177.4 & 49.2 & 198.5 & 456.3 & & 278.2 & 343.8 & 287.1 & 77.7 & 74.22 \\
\hline 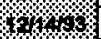 & $15: 40$ & -9.764 & .10 .1 & -0.9 & 1.3 & -26.6 & -18.7 & -5.1 & 8.0 & 343.5 & 179.1 & 51.0 & 201.1 & 458.4 & & 279.1 & 343.8 & 209.7 & 75.8 & 74.22 \\
\hline 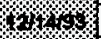 & $15: 50$ & 0.7 .767 & .12 .7 & -4.4 & -1.3 & -29.2 & -20.8 & .7 .7 & -13.2 & 342.7 & 181.7 & 51.0 & 204.6 & 459.0 & & 280.8 & 345.6 & 269.7 & 75.8 & 71.62 \\
\hline $82 \% 1 \%$ & 16:00 & -1.760 & .13 .6 & .7 .8 & -4.8 & -30.1 & .23 .2 & -11.2 & .16 .7 & 287.1 & 181.7 & 49.2 & 206.3 & 458.1 & & 282.6 & 347.3 & 209.7 & 74.2 & 69.92 \\
\hline $82 \% 1 / 4$ & 16:10 & -4.743 & -13.6 & -10.4 & .7 .4 & -31.0 & -26.8 & -15.5 & .18 .4 & 100.9 & 150.5 & 49.2 & 207.2 & 458.1 & & 283.5 & 347.4 & 270.6 & 73.4 & 68.22 \\
\hline 822161 & $16: 20$ & -1.736 & -15.3 & .13 .9 & -10.0 & -33.8 & -27.5 & -18.2 & .21 .0 & 3.0 & 31.7 & 49.2 & 208.1 & 459.0 & & 283.5 & 347.4 & 271.5 & 71.6 & 67.42 \\
\hline
\end{tabular}


Table 5. Tensiometer Data

\begin{tabular}{|c|c|c|c|c|c|c|c|c|c|c|c|c|c|c|c|c|c|c|c|c|}
\hline & & & & & & & & & Tens & Iometer 0 & ata & & & & & & & & & \\
\hline & & Test & \begin{tabular}{|c|} 
Soll \\
Molsture \\
Tension \\
$\left(\mathrm{em} \mathrm{H}_{2} \mathrm{O}\right)$ \\
\end{tabular} & $\begin{array}{c}\text { Soll } \\
\text { Moisture } \\
\text { Tension } \\
\left(\mathrm{Cm} \mathrm{H}_{2} \mathrm{O}\right)\end{array}$ & $\begin{array}{c}\text { Soll } \\
\text { Moisture } \\
\text { Tension } \\
\left(\mathrm{Cm} \mathrm{H}_{2} \mathrm{O}\right)\end{array}$ & $\begin{array}{c}\text { Soil } \\
\text { Moisture } \\
\text { Tension } \\
\left(\mathrm{Cm} \mathrm{H}_{2} \mathrm{O}\right)\end{array}$ & $\begin{array}{c}\text { Soll } \\
\text { Moisture } \\
\text { Tension } \\
\left(\mathrm{Cm} \mathrm{H}_{2} \mathrm{O}\right)\end{array}$ & $\begin{array}{c}\text { Soll } \\
\text { Moisture } \\
\text { Tension } \\
\left(\mathrm{em} \mathrm{H}_{2} \mathrm{O}\right)\end{array}$ & $\begin{array}{c}\text { Soil } \\
\text { Molsture } \\
\text { Tension } \\
\left(e \mathrm{H}_{2} \mathrm{O}\right)\end{array}$ & $\begin{array}{c}\text { Soll } \\
\text { Moisture } \\
\text { Tension } \\
\left(\mathrm{em} \mathrm{H}_{2} \mathrm{O}\right)\end{array}$ & $\begin{array}{c}\text { Soll } \\
\text { Moisture } \\
\text { Tension } \\
\left(\mathrm{Cm} \mathrm{H}_{2} \mathrm{O}\right) \\
\end{array}$ & $\begin{array}{c}\text { Soil } \\
\text { Moisture } \\
\text { Tension } \\
\left(\mathrm{cm} \mathrm{H}_{2} \mathrm{O}\right)\end{array}$ & $\begin{array}{c}\text { Soll } \\
\text { Moisture } \\
\text { Tenslon } \\
\left(\mathrm{cm} \mathrm{H}_{2} \mathrm{O} \text { ) }\right.\end{array}$ & $\begin{array}{c}\text { Soil } \\
\text { Moisture } \\
\text { Tension } \\
\left(\mathrm{cm} \mathrm{H}_{2} \mathrm{O}\right)\end{array}$ & $\begin{array}{c}\text { Soll } \\
\text { Moisture } \\
\text { Tension } \\
\left(\mathrm{em} \mathrm{H}_{2} \mathrm{O}\right)\end{array}$ & $\begin{array}{c}\text { soll } \\
\text { moisture } \\
\text { Tension } \\
\left(\mathrm{cm} \mathrm{H}_{2} \mathrm{O}\right)\end{array}$ & $\begin{array}{c}\text { Soll } \\
\text { Molsture } \\
\text { Tenslon } \\
\left.\text { (em } \mathrm{H}_{2} \mathrm{O}\right) \\
\end{array}$ & $\begin{array}{c}\text { Soll } \\
\text { Molsture } \\
\text { Tension } \\
\left(\mathrm{Cm} \mathrm{H}_{2} \mathrm{O}\right) \\
\end{array}$ & $\begin{array}{c}\text { Soll } \\
\text { Moisture } \\
\text { Tension } \\
\left(\mathrm{em} \mathrm{H} \mathrm{H}_{2} \mathrm{O}\right) \\
\end{array}$ & $\begin{array}{c}\text { Soll } \\
\text { Molsture } \\
\text { Tenslon } \\
\left(\mathrm{em} \mathrm{H}_{2} \mathrm{O}\right) \\
\end{array}$ \\
\hline Date & Time & Days' & $C 1^{\prime \prime}$ & $B 1^{*}$ & $A 1^{\circ}$ & $\mathrm{C2}^{\mathrm{b}}$ & $B 2^{\mathrm{b}}$ & $A 2^{b}$ & $\mathrm{C3}^{\mathrm{C}}$ & $\mathrm{B3}^{\mathrm{C}}$ & $\mathrm{A3}^{\mathrm{C}}$ & $\mathrm{CA}^{\mathrm{d}}$ & $\mathrm{BA}^{\mathrm{d}}$ & $\mathrm{AS}^{\mathrm{d}}$ & $C 5^{\circ}$ & $B 5^{\circ}$ & $A 5^{\circ}$ & $c \sigma^{\prime}$ & $86^{\prime}$ & $A \mathbf{B}^{\prime}$ \\
\hline \%o & 18:30 & -1.729 & .15 .3 & .14 .8 & -12.6 & .33 .6 & -30.1 & -20.8 & .22 .7 & -12.0 & 0.5 & 49.2 & 210.7 & 460.8 & & 285.3 & 348.3 & 272.5 & 71.7 & 68.52 \\
\hline $40.4 \times 1$ & $16: 40$ & -1.722 & -17.1 & -18.2 & $\begin{array}{l}-13.5 \\
\end{array}$ & -36.2 & .33 .5 & .22 .5 & .24 .5 & .17 .3 & .8 .8 & 47.5 & 210.7 & 459.8 & & 285.3 & 348.2 & 273.3 & 69.8 & 65.72 \\
\hline $14,4+4$ & 16:50 & -1.715 & -17.8 & -18.2 & -16.1 & -37.0 & -33.5 & .28 .0 & -28.8 & .22 .6 & .16 .0 & 47.5 & 209.0 & 460.8 & & 285.3 & 350.1 & 271.6 & 69.1 & 83.12 \\
\hline \%24.4\% & $17: 00$ & -1.708 & -17.8 & -17.3 & .17 .0 & -37.0 & .33 .5 & .26 .9 & -28.8 & .24 .4 & .20 .3 & 46.8 & 205.6 & 461.7 & & 282.8 & 351.0 & 270.8 & 67.4 & 62.32 \\
\hline 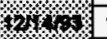 & $17: 10$ & -1.701 & $\begin{array}{l}-17.9 \\
\end{array}$ & -19.8 & -17.8 & .37 .9 & .38 .1 & .28 .8 & -30.5 & .28 .8 & .25 .5 & 447.5 & 203.0 & 461.8 & & 281.8 & 351.0 & 271.7 & 67.4 & 61.42 \\
\hline 30,440 & $17: 20$ & -1.694 & .17 .9 & -20.8 & .19 .6 & .38 .8 & .36 .9 & .30 .4 & -34.0 & -31.4 & -28.1 & 17.8 & 202.1 & 4627 & & 281.8 & 351.9 & 270.0 & 65.7 & 58.92 \\
\hline 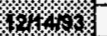 & $17: 30$ & -1.687 & -19.7 & -21.7 & -19.6 & -40.5 & .37 .8 & -32.1 & .34 .8 & -35.0 & -32.5 & -6.6 & 140.6 & 460.9 & & 282.0 & 351.1 & 270.0 & 63.9 & 58.92 \\
\hline \%2, & $17: 40$ & -1.681 & .20 .5 & .22 .5 & -19.6 & -41.4 & -38.6 & -33.8 & -37.4 & -37.6 & -35.9 & -18.8 & 54.8 & 460.1 & & 281.1 & 349.4 & 269.2 & 64.0 & 55.52 \\
\hline 10.1..4. & $17: 50$ & -1.074 & .20 .5 & .22 .5 & .20 .5 & .41 .4 & -39.5 & -35.6 & .37 .4 & -38.5 & .38 .5 & -22.3 & 10.6 & 459.2 & & 280.3 & 348.6 & 288.3 & 62.2 & 54.62 \\
\hline $424 \times$ & 18:00 & -1.667 & -21.4 & .22 .5 & .22 .2 & .41 .4 & -41.2 & .35 .6 & -40.0 & -41.2 & -39.4 & -25.8 & 9.8 & 459.3 & & 279.5 & 346.9 & 267.5 & 60.5 & 52.82 \\
\hline 144614 & 18:10 & -1.600 & -21.4 & -24.3 & .21 .3 & .41 .4 & -41.2 & -38.2 & -40.8 & -42.0 & -42.9 & -34.5 & 8.3 & 459.3 & & 279.5 & 348.7 & 287,5 & 60.5 & 52.12 \\
\hline $28 \%$ & $18: 20$ & -1.653 & .21 .4 & -24.3 & .23 .1 & .42 .2 & -42.1 & -39.1 & -41.7 & -44.7 & -42.9 & -41.5 & 1.1 & 459.3 & & 278.7 & 347.8 & 287.6 & 59.7 & 52.12 \\
\hline 827.44 & $18: 30$ & -1.646 & -24.4 & -24.3 & -23.1 & -42.2 & -41.2 & -40.8 & .44 .3 & -44.7 & -45.5 & -43.2 & -25.8 & 455.8 & & 277.8 & 347.9 & 267.6 & 58.0 & 52.12 \\
\hline$\% 2 \%$. & $18: 40$ & -1.639 & -21.4 & -24.3 & .23 .1 & -43.1 & .43 .8 & .41 .7 & .44 .3 & -46.4 & -45.5 & -45.0 & -44.8 & 427.8 & & 279.6 & 347.8 & 267.6 & 56.3 & 51.32 \\
\hline rex 4 . & $18: 50$ & -1.632 & -21.4 & -25.1 & -23.9 & -44.0 & -43.8 & .42 .6 & .45 .2 & -47.3 & -47.2 & -48.5 & -52.7 & 360.3 & & 279.6 & 348.8 & 287.7 & 55.4 & 48.72 \\
\hline $84.4 \times$ & $18: 00$ & -1.625 & -22.3 & -25.1 & -23.9 & .44 .8 & .44 .7 & .42 .8 & -46.0 & -48.2 & -47.2 & -48.5 & -56.1 & 200.8 & & 279.7 & 348.0 & 269.4 & 55.4 & 49.62 \\
\hline $40.4 \%$ & $18: 10$ & -1.618 & -21.4 & -25.1 & .23 .9 & .44 .0 & -44.7 & .44 .3 & -47.8 & -48.2 & -48.9 & -51.1 & -57.0 & 0.1 & & 279.7 & 348.0 & 268.8 & 52.8 & 49.62 \\
\hline $1240 \%$ & $19: 20$ & -1.611 & -23.1 & -25.1 & .24 .8 & .44 .8 & -45.5 & -44.3 & -47.8 & -49.1 & -50.7 & -51.1 & -59.6 & 0.1 & & 279.7 & 348.8 & 270.3 & 51.9 & 47.92 \\
\hline (21) & 18.30 & -1.604 & -23.1 & -26.9 & -23.9 & -44.8 & -45.5 & -43.4 & -48.6 & -50.0 & -50.7 & -52.0 & -63.1 & -0.8 & & 279.7 & 348.0 & 270.4 & 51.1 & 48.72 \\
\hline 8. & $19: 40$ & -1.697 & -23.1 & -26.9 & -24.8 & .44 .8 & .46 .4 & .44 .3 & -48.6 & .50 .8 & 50.7 & -52.9 & -64.8 & -13.0 & & 280.6 & 348.0 & 268.6 & 51.1 & 48.82 \\
\hline 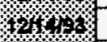 & $21: 10$ & -1.536 & -24.0 & -28.6 & .27 .4 & -47.4 & -47.2 & -47.8 & .51 .2 & -52.6 & -78.4 & -57.2 & -73.5 & -47.2 & & 280.7 & 343.8 & 268.7 & 42.4 & 43.62 \\
\hline 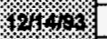 & $21: 20$ & -1.528 & -24.0 & -28.6 & -28.5 & -47.4 & -47.2 & -46.8 & .52 .1 & -54.4 & -79.3 & -59.0 & .74 .3 & -49.0 & & 281.5 & 344.7 & 268.7 & 41.6 & 42.82 \\
\hline 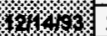 & $21: 30$ & -1.621 & -24.0 & .28 .6 & -27.4 & -47.4 & -47.2 & -47.8 & -52.1 & -54.4 & -79.3 & -57.2 & .78 .1 & -49.8 & & 280.7 & 344.7 & 268.7 & 40.7 & 42.82 \\
\hline (2) & $21: 40$ & -1.614 & .24 .0 & .27 .7 & -27.4 & -47.4 & -48.1 & -48.7 & .52 .1 & -55.3 & -81.0 & -59.0 & .75 .2 & -50.7 & & 281.6 & 343.0 & 269.6 & 40.7 & 41.82 \\
\hline $47,4,4$ - & $21: 50$ & -1.507 & -24.0 & -28.6 & .27 .4 & .47 .4 & .49 .0 & -47.8 & .52 .1 & -55.3 & -81.9 & -59.8 & .78 .1 & .50 .7 & & 282.4 & 342.1 & 268.8 & 39.8 & 41.82 \\
\hline 124 , & $22: 00$ & -1.500 & -24.0 & -28.6 & -28.5 & -47.4 & -49.8 & $-47,8$ & .52 .1 & .56 .1 & -81.9 & -60.7 & .76 .9 & .52 .5 & & 282.4 & 341.3 & 268.8 & 39.8 & 41.82 \\
\hline 121,1 & $22: 10$ & -1.493 & .24 .0 & .28 .6 & .27 .4 & -46.6 & -49.8 & -48.7 & .52 .1 & -55.3 & -81.8 & .62 .5 & .76 .8 & .51 .6 & & 282.5 & 339.8 & 288.8 & 38.1 & 39.42 \\
\hline 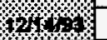 & $22: 20$ & -1.486 & -24.0 & .28 .6 & -27.4 & .47 .4 & -49.0 & -47.8 & -52.1 & -56.1 & -81.9 & -61.6 & .76 .9 & -53.4 & & 282.5 & 337.8 & 288.8 & 39.0 & 39.42 \\
\hline 121,1 & $22: 30$ & -1.479 & .23 .1 & .28 .6 & -27.4 & -48.3 & -48.1 & -48.7 & .52 .1 & -56.1 & -81.9 & -61.6 & .76 .9 & .53 .4 & & 281.6 & 336.1 & 266.8 & 30.4 & 37.62 \\
\hline $127 \%$ & $22: 40$ & -1.072 & .24 .0 & .28 .6 & .27 .4 & .47 .4 & -48.1 & -48.7 & -52.1 & -56.1 & -82.8 & .62 .5 & .76 .9 & .53 .4 & & 281.6 & 334.4 & 288.8 & 36.4 & 35.12 \\
\hline $4 x+4 \%$ & $22: 50$ & -1.465 & .24 .0 & .28 .6 & -27.4 & -47.4 & -19.8 & -47.8 & -52.1 & -56.1 & -81.8 & -62.5 & -76.9 & -53.4 & & 280.7 & 330.9 & 268.7 & 36.4 & 33.32 \\
\hline 14.4 .4 & $23: 00$ & -1.458 & .24 .0 & -28.6 & -26.5 & -47.4 & -49.0 & -48.7 & -52.1 & -55.3 & -83.6 & -62.5 & -78.9 & -54.3 & & 279.0 & 324.8 & 269.7 & 33.8 & 31.62 \\
\hline$\%$ & $23: 10$ & .1 .451 & -24.0 & -28.6 & -27.4 & -47.4 & -49.0 & -48.7 & -52.1 & -56.1 & .83 .6 & -62.5 & -76.9 & -54.3 & & 272.0 & 310.2 & 287.9 & 34.6 & 30.82 \\
\hline (x) & $23: 20$ & -1.444 & -23.1 & -29.5 & -27.4 & -48.6 & -48.1 & -47.8 & -52.9 & .56 .1 & .83 .6 & -62.5 & -76.8 & -54.3 & & 261.6 & 281.7 & 288.8 & 32.8 & 29.02 \\
\hline $6281 \%$ & 23:30 & -1.437 & -24.0 & -30.3 & .27 .4 & -47.4 & -49.8 & -48.7 & -52.9 & .56 .1 & -85.4 & -61.6 & .76 .9 & -56.0 & & 232.8 & 228.2 & 268.8 & 33.8 & 27.32 \\
\hline 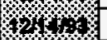 & $23: 40$ & -1.431 & -24.0 & -28.6 & -27.4 & -47.4 & -49.0 & -47.8 & -52.9 & .56 .1 & .85 .4 & -60.7 & -76.8 & -56.0 & & 170.9 & 141.8 & 268.8 & 33.8 & 25.82 \\
\hline (2) 27, & $23: 50$ & -1.424 & -24.0 & -28.6 & .27 .4 & -47.4 & -49.0 & -48.7 & -52.1 & -56.1 & .85 .4 & -59.8 & -76.9 & .56 .9 & & 53.3 & 30.5 & 288.8 & 32.8 & 24.72 \\
\hline 8 & 0.00 & -1.417 & -24.0 & .28 .6 & -27.4 & -47.4 & -49.8 & -48.7 & -52.1 & .58 .1 & .84 .5 & -60.7 & .76 .9 & -56.0 & & 16.7 & 9.8 & 268.8 & 33.8 & 23.02 \\
\hline
\end{tabular}


Table 5. Tensiometer Data

\begin{tabular}{|c|c|c|c|c|c|c|c|c|c|c|c|c|c|c|c|c|c|c|c|c|}
\hline & & & & & & & & & Ten & siometer & Data & & & & & & & & & \\
\hline & & Test & 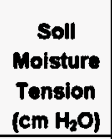 & $\begin{array}{c}\text { Soll } \\
\text { Moisture } \\
\text { Tansion } \\
\left(\mathrm{Cm}_{2} \mathrm{O} \text { ) }\right. \\
\end{array}$ & 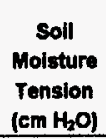 & $\begin{array}{c}\text { Soll } \\
\text { Moisture } \\
\text { Tansion } \\
\left(\mathrm{cm} \mathrm{H}_{2} \mathrm{O}\right) \\
\end{array}$ & $\begin{array}{c}\text { Soll } \\
\text { Moisture } \\
\text { Tension } \\
\left(\mathrm{cm} \mathrm{H}_{2} \mathrm{O}\right) \\
\end{array}$ & $\begin{array}{c}\text { Soll } \\
\text { Moisture } \\
\text { Tension } \\
\left(\mathrm{Cm} \mathrm{H}_{2} \mathrm{O}\right) \\
\end{array}$ & $\begin{array}{c}\text { Soll } \\
\text { Molsture } \\
\text { Tension } \\
\left(\mathrm{C} \mathrm{C}_{2} \mathrm{O}\right) \\
\end{array}$ & 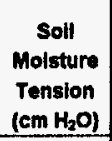 & $\begin{array}{c}\text { Soll } \\
\text { Molsture } \\
\text { Tension } \\
\left(\mathrm{Cm} \mathrm{H}_{2} \mathrm{O}\right) \\
\end{array}$ & $\begin{array}{c}\text { Soll } \\
\text { Molsture } \\
\text { Tansion } \\
\left(\mathrm{cm} \mathrm{H}_{2} \mathrm{O}\right) \\
\end{array}$ & $\begin{array}{c}\text { Soll } \\
\text { Molsture } \\
\text { Tension } \\
\left(\mathrm{Cm} \mathrm{H}_{2} \mathrm{O}\right) \\
\end{array}$ & $\begin{array}{c}\text { Soll } \\
\text { Molsture } \\
\text { Tension } \\
\text { (cm HzO) }\end{array}$ & $\begin{array}{c}\text { Soll } \\
\text { Moisture } \\
\text { Tonsion } \\
\left(\mathrm{cm} \mathrm{H}_{2} \mathrm{O}\right) \\
\end{array}$ & $\begin{array}{c}\text { Soll } \\
\text { Molstume } \\
\text { Tenslon } \\
\left(\mathrm{Cm} \mathrm{H}_{2} \mathrm{O}\right)\end{array}$ & $\begin{array}{c}\text { Soll } \\
\text { Molsture } \\
\text { Tenslon } \\
\left(\mathrm{Cm} \mathrm{H}_{2} \mathrm{O}\right) \\
\end{array}$ & $\begin{array}{c}\text { Soll } \\
\text { Moisture } \\
\text { Tension } \\
\left(\mathrm{em}_{2} \mathrm{O} \text { ) }\right. \\
\end{array}$ & $\begin{array}{c}\text { Soll } \\
\text { Molsture } \\
\text { Tonsion } \\
\left(\mathrm{cm} \mathrm{H}_{2} \mathrm{O}\right) \\
\end{array}$ & $\begin{array}{c}\text { soll } \\
\text { Molsture } \\
\text { Tension } \\
\left(\mathrm{Cm} \mathrm{H}_{2} \mathrm{O}\right) \\
\end{array}$ \\
\hline Date & Time & Days ${ }^{1}$ & $\mathrm{C1}$ & $B 1^{2}$ & $A 1^{*}$ & $\mathrm{Cl}^{\mathrm{b}}$ & $\mathrm{B2}^{\mathrm{b}}$ & $A 2^{b}$ & $\mathrm{CB}^{\mathrm{C}}$ & $\mathrm{B3}^{\mathrm{c}}$ & $A 3^{c}$ & $\mathrm{C}^{\mathrm{d}}$ & $B 4^{\circ}$ & $A 4^{6}$ & $C 5^{\circ}$ & $\mathrm{BS}^{\circ}$ & $A 5^{\circ}$ & $\mathrm{Cb}^{f}$ & $B 6^{\prime}$ & $A 6^{\prime}$ \\
\hline 1 34 & $0: 10$ & -1.410 & .24 .0 & .28 .6 & -27.4 & -45.7 & -49.8 & -48.7 & .52 .8 & .56 .1 & -85.4 & -61.6 & -77.8 & -56.9 & & 15.8 & 2.9 & 268.8 & 32.0 & 22.22 \\
\hline 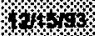 & $0: 20$ & -1.403 & .24 .0 & -28.6 & -27.4 & -47.4 & -49.8 & -47.8 & .52 .8 & .56 .1 & -85.4 & -62.5 & .76 .8 & -56.9 & & 11.4 & -1.4 & 288.8 & 31.2 & 21.32 \\
\hline \% & 0.30 & -1.396 & .24 .0 & -28.6 & .27 .4 & -47.4 & -50.7 & -49.5 & -52.1 & .56 .1 & -85.4 & -81.6 & -76.9 & .56 .9 & & 10.6 & -4.0 & 267.8 & 30.3 & 21.32 \\
\hline $1 \%$ & $0: 40$ & -1.389 & .24 .0 & -28.6 & .27 .4 & -47.4 & -49.8 & -49.5 & .52 .1 & .54 .4 & 85.4 & -62.5 & -77.8 & .56 .9 & & 8.8 & -6.6 & 284.4 & 29.4 & 18.72 \\
\hline 121,6 & 0.50 & -1.382 & -24.8 & -28.8 & .27 .4 & -47.4 & -50.7 & -48.7 & .52 .8 & .58 .1 & -86.2 & -61.8 & -76.9 & -57.8 & & 8.8 & -0.2 & 200.1 & 29.4 & 17.82 \\
\hline $821 \% 4$ & $1: 00$ & -1.376 & -24.0 & -28.6 & .27 .4 & -49.2 & -50.7 & -48.7 & -52.8 & .58 .1 & 85.4 & -61.6 & -78.6 & .56 .8 & & 7.1 & -10.0 & 254.0 & 29.4 & 17.92 \\
\hline 8344 & $1: 10$ & -1.368 & -24.0 & .28 .6 & .27 .4 & -47.4 & -49.8 & -48.7 & -52.1 & -58.1 & 86.2 & -61.8 & -77.8 & .56 .9 & & 7.1 & -10.0 & 248.1 & 29.4 & 17.92 \\
\hline 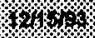 & $1: 20$ & -1.361 & -24.0 & .28 .8 & .27 .4 & -47.4 & -49.8 & -48.7 & -52.8 & .58 .1 & -86.2 & -61.6 & .77 .8 & .57 .8 & & 7.1 & -10.0 & 233.0 & 29.4 & 17.92 \\
\hline 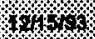 & $1: 30$ & -1.364 & -24.0 & .28 .6 & .27 .4 & -47.4 & -49.8 & -48.7 & .52 .9 & .58 .1 & -88.0 & -60.7 & .76 .9 & .56 .9 & & 7.1 & -10.9 & 218.1 & 29.4 & 17.82 \\
\hline $10+4+8$ & $1: 40$ & -1.347 & -24.0 & .28 .6 & .27 .4 & -47.4 & -50.7 & -48.7 & -52.1 & .58 .1 & -87.1 & -60.7 & -76.9 & -59.5 & & 8.2 & -10.8 & 197.1 & 29.4 & 17.92 \\
\hline$\gamma_{31}, 4$ & $1: 50$ & -1.340 & -24.0 & .28 .6 & .27 .4 & -48.3 & -49.8 & -48.7 & .52 .8 & 58.1 & 88.8 & -61.6 & -77.8 & .59 .5 & & 5.4 & -12.6 & 170.8 & 28.6 & 17.82 \\
\hline (5.3. & 200 & $\begin{array}{c}-1.3333 \\
\end{array}$ & .23 .1 & .28 .6 & .28 .3 & -47.4 & -49.8 & .48 .7 & -52.1 & .56 .1 & -88.0 & -60.7 & -76.8 & .59 .5 & & 4.5 & -12.8 & 142.8 & 27.7 & 17.02 \\
\hline $121 \%$ & $2: 10$ & -1.326 & .23 .1 & -28.6 & .27 .4 & -47.4 & -50.7 & .50 .4 & -52.8 & .58 .1 & -88.8 & -61.6 & -77.8 & .59 .5 & & 4.5 & -13.5 & 112.3 & 28.8 & 17.82 \\
\hline 421640 & 220 & -1.319 & .24 .0 & .28 .6 & -27.4 & -47.4 & -51.6 & .48 .7 & .52 .9 & -56.1 & -88.8 & -61.6 & -77.8 & .58 .6 & & 6.2 & -12.6 & 85.2 & 26.8 & 17.92 \\
\hline 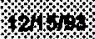 & $2: 30$ & -1.312 & .24 .8 & -29.5 & .27 .4 & -47.4 & -49.8 & .48 .7 & .51 .2 & -56.1 & -88.8 & .62 .5 & .77 .8 & -59.5 & & 5.4 & -13.5 & 61.5 & 26.8 & 17.82 \\
\hline \% & $2: 40$ & -1.306 & .24 .0 & .28 .6 & .27 .4 & -47.4 & .50 .7 & 49.5 & .52 .8 & -56.1 & -88.8 & .62 .5 & $-\pi 7.8$ & -58.5 & & 4.5 & -13.5 & 49.3 & 25.1 & 17.92 \\
\hline 4 (2) & 2.50 & -1.290 & .24 .0 & -28.6 & -27.4 & -45.7 & .50 .7 & -49.5 & .52 .8 & -56.1 & -88.8 & -82.5 & .79 .5 & .59 .5 & & 3.6 & -14.3 & 41.4 & 26.0 & 16.12 \\
\hline 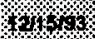 & $3: 00$ & -1.292 & .24 .0 & -28.6 & -27.4 & -47.4 & .50 .7 & -49.5 & .52 .9 & -56.1 & -89.7 & -62.5 & .78 .6 & .60 .4 & & 4.5 & -14.3 & 37.9 & 28.0 & 13.62 \\
\hline \% 3 r 6 \% & $3: 10$ & -1.285 & .24 .0 & -27.7 & -27.4 & -47.4 & .50 .7 & .49 .5 & .52 .9 & -56.1 & -88.8 & -62.5 & -80.4 & -60.4 & & 3.6 & -13.5 & 37.0 & 22.5 & 11,02 \\
\hline 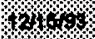 & $3: 20$ & $\begin{array}{l}-1.278 \\
\end{array}$ & .24 .0 & -28.6 & -27.4 & -47.4 & 49.0 & -48.7 & .52 .9 & -56.1 & -89.7 & -61.6 & -80.4 & .59 .5 & & 4.5 & -14.3 & 37.1 & 21.6 & 7.52 \\
\hline $121 \% 8$ & $3: 30$ & -1.271 & .24 .0 & -28.6 & -27.4 & -47.4 & .50 .7 & -49.5 & .52 .9 & -56.1 & -88.8 & -59.8 & 80.4 & .60 .4 & & 5.4 & .14 .3 & 36.2 & 16.4 & 4.12 \\
\hline $82 \%$ & $3: 40$ & -1.264 & .24 .0 & -28.6 & -28.3 & -48.3 & 49.8 & -49.5 & .52 .9 & .56 .1 & -89.7 & -60.7 & .78 .6 & .59 .5 & & 4.5 & -14.3 & 35.3 & 12.0 & -0.18 \\
\hline 184,4 & $3: 50$ & -1.267 & .24 .0 & .28 .6 & -26.5 & -47.4 & -49.8 & -48.7 & .52 .9 & .58 .1 & -89.7 & -59.8 & .78 .6 & -60.4 & & 3.6 & -14.3 & 36.2 & 6.8 & 4.48 \\
\hline 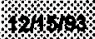 & $4: 00$ & -1.250 & -24.0 & .28 .6 & -27.4 & -47.4 & .50 .7 & -48.7 & -53.8 & .57 .0 & .89 .7 & -62.5 & -80.4 & -58.6 & & 3.6 & -13.5 & 33.6 & 1.0 & .7 .98 \\
\hline 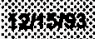 & $4: 10$ & -1.243 & .24 .0 & .28 .6 & .27 .4 & -47.4 & -49.0 & -48.7 & .52 .9 & .58 .1 & -89.7 & -62.5 & -78.6 & -60.4 & & 4.5 & .13 .5 & 33.6 & -4.5 & -12.28 \\
\hline 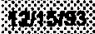 & $4: 20$ & -1.236 & -24.0 & -27.7 & .27 .4 & -47.4 & -49.8 & -49.5 & .52 .1 & .56 .1 & -89.7 & -62.5 & -78.6 & -59.5 & & 4.5 & .13 .5 & 34.4 & -8.8 & -14.88 \\
\hline $87 \% 6$ & 4:30 & -1.229 & -24.0 & .29 .5 & .28 .5 & -47.4 & -50.7 & -48.7 & .52 .1 & -56.1 & -89.7 & -60.7 & .78 .6 & -80.4 & & 4.5 & -14.3 & 32.7 & -14.9 & -16.58 \\
\hline $1 \% 10 \%$ & $4: 40$ & -1.222 & -23.1 & .29 .5 & .27 .4 & -47.4 & -49.0 & .48 .7 & -52.9 & .57 .0 & .01 .5 & -61.6 & .79 .5 & -59.5 & & 4.5 & -14.3 & 33.6 & .19 .3 & -19.88 \\
\hline 121,11 & $4: 50$ & -1.216 & .24 .8 & .28 .6 & .27 .4 & -47.4 & -49.8 & -49.5 & -52.9 & .56 .1 & .82 .3 & .82 .5 & .79 .5 & -59.5 & & 3.6 & -14.3 & 33.6 & .22 .8 & .23 .46 \\
\hline 1214 & $5: 00$ & -1.208 & -23.1 & -28.6 & .27 .4 & -47.4 & -50.7 & -48.7 & .52 .9 & .56 .1 & .01 .5 & -62.5 & -80.4 & .59 .5 & & 4.5 & -14.3 & 33.6 & .22 .8 & .23 .48 \\
\hline 8464 & $5: 10$ & -1.201 & -24.0 & -28.6 & -27.4 & -47.4 & -50.7 & -49.5 & -53.8 & -58.1 & .82 .3 & .62 .5 & .79 .5 & -60.4 & & 4.5 & -14.3 & 33.6 & -28.2 & .25 .88 \\
\hline $42+20$ & $5: 20$ & -1.194 & .24 .0 & -28.6 & -27.4 & .47 .4 & -50.7 & -48.7 & -52.9 & -58.1 & -01.5 & -62.5 & .79 .5 & -80.4 & & 5.4 & -14.3 & 33.6 & -26.2 & -26.88 \\
\hline $1446 \%$ & $5: 30$ & -1.187 & .24 .0 & -26.9 & -27.4 & -47.4 & .50 .7 & -50.4 & -52.9 & -57.0 & -91.5 & -60.7 & .78 .5 & .58 .6 & & 3.6 & -14.3 & 33.8 & .29 .7 & .28 .88 \\
\hline 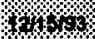 & $5: 40$ & -1.181 & .24 .8 & -28.6 & -27.4 & .47 .4 & .50 .7 & -49.5 & -52.9 & -56.1 & -81.5 & .61 .6 & 80.4 & -59.5 & & 4.5 & -14.3 & 33.6 & .29 .7 & -29.48 \\
\hline $12,4.0$ & 5:50 & -1.174 & .24 .0 & -28.6 & -28.3 & -47.4 & .49 .8 & -49.5 & -52.9 & .56 .1 & -80.6 & -61.6 & -80.4 & -60.4 & & 4.5 & -14.3 & 32.7 & -29.7 & 30.28 \\
\hline $84 \%$ & 6:00 & -1.167 & .23 .1 & -28.6 & -27.4 & -47.4 & .50 .7 & .50 .4 & .53 .8 & -58.1 & -82.3 & -60.7 & .78 .6 & -80.4 & & 0.2 & -44.3 & 34.4 & 31.5 & -30.28 \\
\hline 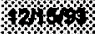 & $6: 10$ & -1.160 & .24 .0 & -28.6 & -27.4 & -46.5 & -49.8 & .48 .7 & .53 .8 & -57.0 & -92.3 & -61.6 & .79 .5 & -60.4 & & 4.5 & -15.2 & 30.1 & -33.2 & -31.18 \\
\hline 82 & $6: 20$ & -1.163 & -24.8 & .28 .6 & -28.3 & $\$ 7.4$ & .50 .7 & -49.5 & .52 .8 & -56.1 & -80.4 & -02.5 & .79 .5 & .59 .5 & & 4.5 & -14.3 & 31.8 & 33.2 & 32.88 \\
\hline
\end{tabular}


Table 5. Tensiometer Data

\begin{tabular}{|c|c|c|c|c|c|c|c|c|c|c|c|c|c|c|c|c|c|c|c|c|}
\hline & & & & & & & & & Ten & siometer & Data & & & & & & & & & \\
\hline & & Test & $\begin{array}{c}\text { Soil } \\
\text { Molsture } \\
\text { Tension } \\
\left(\mathrm{cm} \mathrm{H}_{2} \mathrm{O}\right) \\
\end{array}$ & $\begin{array}{c}\text { Soll } \\
\text { Molsture } \\
\text { Tenslon } \\
\left(\mathrm{cm} \mathrm{H}_{2} \mathrm{O}\right)\end{array}$ & $\begin{array}{c}\text { Solf } \\
\text { Moisture } \\
\text { Tension } \\
\left(\mathrm{Cm}_{2} \mathrm{O}\right) \\
\end{array}$ & $\begin{array}{c}\text { Soll } \\
\text { Moisture } \\
\text { Tension } \\
\left(\mathrm{Cm} \mathrm{H}_{2} \mathrm{O}\right) \\
\end{array}$ & $\begin{array}{c}\text { Soil } \\
\text { Moisture } \\
\text { Tension } \\
\left(\mathrm{cm} \mathrm{H}_{2} \mathrm{O}\right)\end{array}$ & 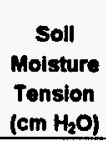 & \begin{tabular}{|c|} 
Soll \\
Moisture \\
Tension \\
(cm H $\mathrm{H}_{2} \mathrm{O}$ ) \\
\end{tabular} & $\begin{array}{c}\text { Soll } \\
\text { Moisture } \\
\text { Tension } \\
\left(\mathrm{Cm} \mathrm{H}_{2} \mathrm{O} \text { ) }\right. \\
\end{array}$ & $\begin{array}{c}\text { Soil } \\
\text { Molsture } \\
\text { Tension } \\
\left(\mathrm{Cm} \mathrm{H}_{2} \mathrm{O}\right) \\
\end{array}$ & $\begin{array}{c}\text { Soll } \\
\text { Moisture } \\
\text { Tension } \\
\left(\mathrm{Cm} \mathrm{H}_{2} \mathrm{O}\right) \\
\end{array}$ & $\begin{array}{c}\text { Soll } \\
\text { Moisture } \\
\text { Tension } \\
\left(\mathrm{Cm}_{2} \mathrm{O} \text { ) }\right. \\
\end{array}$ & $\begin{array}{c}\text { Soll } \\
\text { Molsture } \\
\text { Tension } \\
\left.\text { (cm } \mathrm{H}_{2} \mathrm{O}\right)\end{array}$ & $\begin{array}{c}\text { Soll } \\
\text { Molsture } \\
\text { Tansion } \\
\left(\mathrm{Cm} \mathrm{H}_{2} \mathrm{O}\right) \\
\end{array}$ & $\begin{array}{c}\text { Soll } \\
\text { Molsture } \\
\text { Tension } \\
\left(\mathrm{cm} \mathrm{H}_{2} \mathrm{O}\right) \\
\end{array}$ & $\begin{array}{c}\text { Soll } \\
\text { Molsture } \\
\text { Tension } \\
\left(\mathrm{Cm} \mathrm{H}_{2} \mathrm{O}\right) \\
\end{array}$ & $\begin{array}{c}\text { Soll } \\
\text { Molsture } \\
\text { Tenslon } \\
\text { (cm } \mathrm{H}_{2} \mathrm{O} \text { ) }\end{array}$ & $\begin{array}{c}\text { Soll } \\
\text { Moisture } \\
\text { Tenslon } \\
\left(\mathrm{cm} \mathrm{H}_{2} \mathrm{O}\right) \\
\end{array}$ & $\begin{array}{c}\text { Soll } \\
\text { Molsture } \\
\text { Tension } \\
\left(\mathrm{Cm} \mathrm{H}_{2} \mathrm{O}\right) \\
\end{array}$ \\
\hline Date & Time & Days ${ }^{1}$ & $C 1^{\prime \prime}$ & $B 1^{2}$ & $\mathrm{A1}^{\mathrm{a}}$ & $\mathrm{C2}^{\mathrm{b}}$ & $\mathbf{B 2}^{\mathbf{b}}$ & $A 2^{b}$ & $\mathrm{C3}^{\mathrm{C}}$ & $\mathrm{B3}^{\mathrm{C}}$ & $\mathbf{A} 3^{\mathbf{c}}$ & $\mathrm{CA}^{\mathrm{d}}$ & $B 4^{d}$ & $A A^{d}$ & $\mathrm{C5}^{\circ}$ & $B 5^{\circ}$ & $A 6^{\circ}$ & $\mathrm{CG}^{\prime}$ & $\mathrm{Bo}^{\prime}$ & $A G^{\prime}$ \\
\hline$\% \%$ & $8: 30$ & -1.146 & -23.1 & -28.8 & -27.4 & .47 .4 & .50 .7 & -48.7 & -53.8 & -56.1 & -91.5 & -62.5 & .78 .5 & 60.4 & & 4.5 & .14 .3 & 30.8 & -34.1 & -32.88 \\
\hline $1010 \%$ & $6: 40$ & -1.139 & -23.1 & -29.5 & -27.4 & -48.3 & .50 .7 & -48.7 & -52.9 & -56.1 & -92.3 & -62.5 & .78 .6 & -60.4 & & 3.8 & .14 .3 & 32.7 & -33.2 & -32.88 \\
\hline 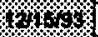 & $6: 50$ & -1.132 & -24.0 & -28.6 & .27 .4 & -47.4 & -50.7 & -48.7 & -52.9 & -56.1 & .92 .3 & .62 .5 & $.78,6$ & -60.4 & & 5.4 & .14 .3 & 31.0 & -34.1 & -33.78 \\
\hline $14 \% 4$ & $7: 00$ & -1.126 & -24.0 & -29.5 & -27.4 & -47.4 & -49.8 & -49.5 & -53.8 & -56.1 & .92 .3 & -82.5 & -80.4 & -60.4 & & 4.5 & .14 .3 & 31.8 & -34.9 & -33.78 \\
\hline $12 \% 1 \%$ & $7: 10$ & -1.118 & -24.8 & -30.3 & -27.4 & .47 .4 & .49 .8 & -48.7 & -52.9 & .56 .1 & .02 .3 & .62 .4 & 80.4 & -60.4 & & 4.5 & .12 .6 & 31.0 & -36.7 & -33.78 \\
\hline 123404 & $7: 20$ & -1.111 & -24.0 & -28.6 & -27.4 & -47.4 & -49.8 & -49.5 & -54.7 & .58 .1 & -92.3 & -64.2 & -80.4 & -60.4 & & 5.4 & -13.5 & 31.0 & -38.7 & -33.78 \\
\hline 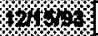 & $7: 30$ & -1.104 & -24.8 & -28.6 & .27 .4 & -47.4 & -50.7 & -48.7 & -52.9 & -56.1 & -92.3 & -62.4 & .79 .5 & -60.4 & & 4.5 & .14 .3 & 31.0 & 35.8 & -33.78 \\
\hline $82 \%$ & $7: 40$ & -1.097 & -24.0 & -28.6 & -28.3 & -47.4 & -50.7 & -48.7 & -53.8 & .57 .0 & .92 .3 & .62 .4 & .78 .6 & -60.4 & & 4.5 & .14 .3 & 31.0 & 36.7 & -33.78 \\
\hline 818480 & $7: 50$ & -1.090 & -23.1 & -28.6 & .27 .4 & -47.4 & -49.8 & -48.7 & -52.8 & .57 .0 & .92 .3 & -62.4 & .79 .5 & -60.4 & & 4.5 & -14.3 & 31.0 & -36.7 & -35.48 \\
\hline 104,40 & $8: 00$ & -1.083 & -24.0 & -28.6 & .27 .4 & -47.4 & -49.8 & -48.7 & -53.8 & .58 .1 & -92.3 & .62 .4 & .79 .5 & -80.4 & & 5.4 & -14.3 & 31.0 & -35.8 & -36.38 \\
\hline $14 \%$ & $8: 10$ & -1.076 & -23.1 & -28.6 & -27.4 & -47.4 & -49.0 & 48.7 & -53.8 & .56 .1 & .92 .3 & .62 .5 & .78 .6 & -60.4 & & 5.4 & .14 .3 & 30.1 & -36.7 & -37.18 \\
\hline 12414.4 & $8: 20$ & -1.069 & -24.0 & -28.6 & .27 .4 & -48.3 & -46.4 & -48.7 & -53.8 & .56 .1 & .92 .3 & .62 .5 & .78 .8 & -59.5 & & 7.1 & -14.3 & 30.1 & 38.7 & -37.18 \\
\hline 12140 & $8: 30$ & -1.062 & -23.1 & -28.6 & .27 .4 & -47.4 & -47.2 & -48.7 & -53.8 & -56.1 & -92.3 & -63.3 & .77 .8 & -59.5 & & 3.6 & .13 .5 & 30.1 & -38.4 & -37.18 \\
\hline 4284,84 & $8: 40$ & -1.066 & -23.1 & -28.6 & .29 .2 & -47.4 & -47.2 & -47.8 & -52.8 & -56.1 & -90.6 & .64 .2 & -76.8 & -59.5 & & 6.2 & -15.2 & 30.1 & 38.4 & -38.08 \\
\hline 82404 & $8: 50$ & -1.049 & -23.1 & -28.6 & -27.4 & -47.4 & .47 .2 & -48.7 & -54.7 & .56 .1 & .92 .3 & .64 .2 & $\begin{array}{r}-78.8 \\
\end{array}$ & -59.5 & & 6.2 & .14 .3 & 30.0 & -38.4 & -38.08 \\
\hline 414 & $9: 00$ & $\begin{array}{r}-1.042 \\
\end{array}$ & -24.0 & -28.6 & -27.4 & -47.4 & -47.2 & -48.7 & -52.1 & -55.2 & .92 .3 & -63.3 & -76.9 & $-\infty 0.4$ & & 6.2 & .14 .3 & 30.0 & -30.3 & -38.98 \\
\hline 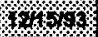 & $8: 10$ & -1.036 & -23.1 & -27.7 & .27 .4 & .47 .4 & .47 .2 & -47.8 & -52.9 & -56.1 & .92 .3 & .65 .1 & -76.8 & -60.4 & & 6.2 & .14 .4 & 30.0 & -30.3 & -38.08 \\
\hline 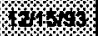 & $9: 20$ & \begin{tabular}{|c|}
-1.028 \\
\end{tabular} & -24.0 & -28.6 & -27.4 & .47 .4 & .47 .2 & -47.8 & -52.8 & -55.2 & .91 .5 & -65.1 & .76 .9 & -60.4 & & 8.2 & .14 .4 & 30.0 & -38.5 & 38.98 \\
\hline $4 \%$ & $9: 30$ & -1.021 & -22.2 & -26.9 & .28 .5 & -47.4 & -47.2 & -47.8 & -52.1 & -55.3 & -91.5 & .64 .2 & -76.8 & -58.6 & & 7.9 & .13 .5 & 30.0 & -39.4 & -38.98 \\
\hline 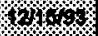 & $9: 40$ & -1.014 & -23.1 & -26.0 & -28.5 & -47.4 & .47 .2 & -46.0 & -52.1 & -56.1 & -92.3 & -65.1 & -76.8 & -58.6 & & 8.2 & .13 .5 & 30.0 & -39.4 & -39.88 \\
\hline 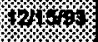 & $9: 50$ & -1.007 & -21.4 & -26.9 & -25.7 & -46.6 & .47 .2 & -46.0 & -52.1 & -55.3 & .92 .3 & .65 .1 & -78.1 & -58.6 & & 7.0 & .13 .5 & 30.0 & -39.4 & -38.88 \\
\hline $81 \% 4 \%$ & $10: 00$ & -1.000 & -22.3 & -27.7 & -25.7 & -47.4 & -47.2 & -46.0 & -52.1 & -55.3 & .92 .3 & .65 .1 & .76 .1 & -57.8 & & 7.0 & -13.5 & 30.0 & -30.4 & -38.98 \\
\hline 1210 & $10: 10$ & 0.993 & -21.4 & -26.8 & .25 .7 & .46 .6 & .45 .5 & -46.9 & -53.0 & -55.3 & -91.5 & -65.1 & -76.9 & -58.6 & & 7.0 & -13.5 & 29.8 & -40.3 & -40.78 \\
\hline 127401 & 10:20 & 0.986 & -21.4 & -26.9 & .28 .5 & -46.6 & -46.4 & -46.0 & -53.0 & -55.3 & -91.5 & -65.1 & .76 .8 & -58.7 & & 7.0 & -11.8 & 29.1 & -40.3 & -39.88 \\
\hline$\% 2 \% 1 \%$ & $10: 30$ & -0.979 & -21.4 & -26.0 & -25.7 & -46.6 & -46.4 & -46.0 & -53.0 & -55.3 & .91 .5 & -65.1 & .76 .1 & -58.7 & & 7.0 & -12.7 & 29.0 & -40.3 & -39.88 \\
\hline 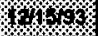 & $10: 40$ & -0.972 & -21.4 & -27.7 & -25.7 & -46.6 & -47.3 & -46.0 & -51.2 & -55.3 & .82 .3 & -85.1 & .75 .2 & -57.8 & & 7.0 & -12.7 & 29.0 & -40.3 & -39.88 \\
\hline 620104 & $10: 50$ & -0.965 & .21 .4 & -26.0 & .25 .7 & -46.6 & -46.4 & -46.0 & .51 .2 & .55 .3 & .81 .5 & -65.1 & .78 .1 & -57.8 & & 7.0 & -12.7 & 29.8 & -39.4 & -40.78 \\
\hline $124 \%, 0$ & $11: 00$ & -0.958 & .21 .4 & -26.0 & .24 .8 & -45.7 & -46.4 & -46.0 & -52.1 & -55.3 & .92 .3 & -65.1 & -78.1 & .57 .8 & & 7.0 & -12.7 & 28.1 & -40.3 & -40.78 \\
\hline 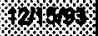 & 11:10 & -0.951 & -21.4 & .26 .0 & .24 .8 & -44.8 & -45.5 & -46.0 & -51.2 & .55 .3 & .92 .3 & -64.2 & .76 .1 & .57 .8 & & 7.0 & .11 .8 & 28.1 & -40.3 & 40.78 \\
\hline $121 \%$ & $11: 20$ & -0.944 & .21 .4 & -25.1 & -23.9 & -44.8 & -45.5 & -46.8 & -51.2 & .55 .3 & -91.5 & -65.1 & .78 .1 & .57 .8 & & 7.0 & -12.7 & 28.1 & -40.3 & 40.78 \\
\hline $8 \%$ & $11: 30$ & -0.937 & -21.4 & -25.1 & -24.8 & -45.7 & -46.4 & -46.9 & .52 .1 & .55 .3 & .92 .3 & 65.1 & .78 .1 & .58 .7 & & 7.0 & -12.7 & 28.1 & -40.3 & -40.78 \\
\hline 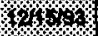 & $11: 40$ & 0.931 & .21 .4 & -26.0 & -24.8 & -44.8 & -45.5 & -46.0 & .51 .2 & -56.1 & -92.3 & -65.1 & .78 .1 & -57.8 & & 6.9 & -12.7 & 28.1 & .40 .3 & -39.98 \\
\hline 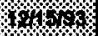 & $11: 50$ & -0.924 & -22.3 & -26.0 & -24.8 & -44.8 & -46.4 & -45.2 & -52.1 & -56.1 & -92.3 & -65.1 & .78 .1 & -57.8 & & 0.8 & -12.8 & 28.1 & -40.3 & -40.78 \\
\hline $40 \%$ & $12: 00$ & -0.917 & -21.4 & -28.0 & -23.9 & -44.8 & .46 .4 & -48.0 & .52 .1 & -56.2 & -92.3 & -64.2 & .77 .0 & -57.8 & & 6.9 & -12.8 & 28.1 & -42.1 & -40.78 \\
\hline$\% 4 \%$ & $12: 10$ & 0.910 & -21.4 & -26.0 & -24.8 & -44.8 & .45 .5 & -46.1 & -51.3 & -55.3 & -92.3 & -65.1 & .77 .0 & -57.8 & & 6.9 & .11 .8 & 28.1 & -42.1 & -40.88 \\
\hline $424 \%$ & $12: 20$ & 0.003 & -21.4 & -25.1 & -29.9 & -46.8 & -45.5 & -45.2 & -52.1 & -55.3 & -92.3 & .04 .2 & .77 .0 & -57.8 & & 6.9 & -12.8 & 28.8 & -41.2 & -40.88 \\
\hline 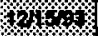 & $12: 30$ & -0.896 & -21.4 & -25.1 & -23.8 & .44 .8 & .45 .5 & -46.1 & -51.3 & -55.3 & -92.3 & .64 .2 & .77 .0 & -57.8 & & 6.8 & -12.8 & 28.8 & -41.2 & -40.88 \\
\hline 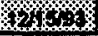 & $12: 40$ & 0.889 & .21 .4 & -25.1 & -23.9 & .44 .8 & .43 .8 & -44.3 & -52.1 & -56.2 & -92.3 & -65.1 & .78 .1 & -57.8 & & 6.0 & -12.8 & 28.0 & -42.1 & -41.68 \\
\hline
\end{tabular}


Table 5. Tensiometer Data

\begin{tabular}{|c|c|c|c|c|c|c|c|c|c|c|c|c|c|c|c|c|c|c|c|c|}
\hline & & & & & & & & & Tens & siometer & Jata & & & & & & & & & \\
\hline & & Test & $\begin{array}{c}\text { Soll } \\
\text { Molsture } \\
\text { Tenslon } \\
\left(\mathrm{Cm} \mathrm{H}_{2} \mathrm{O}\right) \\
\end{array}$ & $\begin{array}{c}\text { Soll } \\
\text { Moisture } \\
\text { Tension } \\
\left(\mathrm{Cm} \mathrm{H}_{3} \mathrm{O}\right) \\
\end{array}$ & $\begin{array}{c}\text { Soll } \\
\text { Moisture } \\
\text { Tension } \\
\left(\mathrm{cm} \mathrm{H}_{2} \mathrm{O}\right) \\
\end{array}$ & $\begin{array}{c}\text { Soll } \\
\text { Molsture } \\
\text { Tension } \\
\left(\mathrm{Cm} \mathrm{H}_{2} \mathrm{O}\right)\end{array}$ & $\begin{array}{c}\text { Soll } \\
\text { Moisture } \\
\text { Tension } \\
\left(\mathrm{Em} \mathrm{H}_{2} \mathrm{O}\right)\end{array}$ & $\begin{array}{c}\text { Soll } \\
\text { Molsture } \\
\text { Tension } \\
\left(\mathrm{cm} \mathrm{H}_{2} \mathrm{O}\right) \\
\end{array}$ & $\begin{array}{c}\text { Soll } \\
\text { Moisture } \\
\text { Tension } \\
\left(\mathrm{Cm} \mathrm{H}_{2} \mathrm{O}\right) \\
\end{array}$ & $\begin{array}{c}\text { Soll } \\
\text { Molsture } \\
\text { Tension } \\
\left(\mathrm{cm} \mathrm{H}_{2} \mathrm{O}\right)\end{array}$ & $\begin{array}{c}\text { Soll } \\
\text { Molsture } \\
\text { Tension } \\
\left(\mathrm{Cm} \mathrm{H}_{2} \mathrm{O}\right) \\
\end{array}$ & $\begin{array}{c}\text { Soll } \\
\text { Moisture } \\
\text { Tension } \\
\left(\mathrm{Cm} \mathrm{H}_{2} \mathrm{O}\right)\end{array}$ & 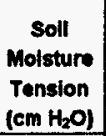 & $\begin{array}{c}\text { Soll } \\
\text { Molsture } \\
\text { Tension } \\
\left(\mathrm{Cm} \mathrm{H}_{2} \mathrm{O}\right) \\
\end{array}$ & $\begin{array}{c}\text { Soll } \\
\text { Molsture } \\
\text { Tenslon } \\
\left(\mathrm{cm} \mathrm{H}_{2} \mathrm{O}\right)\end{array}$ & 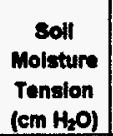 & $\begin{array}{c}\text { soll } \\
\text { Molsture } \\
\text { Tension } \\
\left(\mathrm{cm} \mathrm{H}_{2} \mathrm{O}\right)\end{array}$ & $\begin{array}{c}\text { Soll } \\
\text { Moisture } \\
\text { Tonslon } \\
\left(\mathrm{em} \mathrm{H}_{2} \mathrm{O}\right)\end{array}$ & $\begin{array}{c}\text { Soll } \\
\text { Molsture } \\
\text { Tension } \\
\left.\text { (cm } \mathrm{H}_{2} \mathrm{O}\right)\end{array}$ & 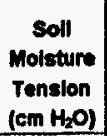 \\
\hline Date & Time & Days ${ }^{1}$ & $\mathrm{C1}^{*}$ & $B 1^{n}$ & $A 1^{2}$ & $\mathrm{C2}^{\mathrm{b}}$ & $\mathrm{B2}^{\mathrm{b}}$ & $A 2^{\mathrm{b}}$ & $c 3^{c}$ & $\mathrm{B3}^{\mathrm{C}}$ & $A 3^{\mathrm{C}}$ & $\mathrm{C}^{\mathrm{d}}$ & $\mathrm{BA}^{\mathrm{d}}$ & $\mathrm{A4}^{\mathrm{d}}$ & $C 5^{\circ}$ & $B 6^{\circ}$ & $A 5^{\circ}$ & $\mathrm{CG}^{\prime}$ & $86^{\prime}$ & $A 6^{\prime}$ \\
\hline Yol 5 \% & 12:50 & -0.882 & .21 .4 & -26.0 & -23.9 & -44.8 & -45.5 & -45.2 & -52.1 & -56.2 & .92 .3 & -86.0 & .76 .1 & .56 .8 & & 6.8 & -11.1 & 28.9 & -42.1 & -41.68 \\
\hline \% 1,0 & $13: \infty$ & 0.876 & -22.3 & -25.1 & .23 .9 & .44 .0 & .44 .7 & -45.2 & .52 .1 & -53.5 & -92.3 & .65 .1 & .77 .0 & .56 .9 & & 0.8 & $.11,8$ & 28.8 & -41.2 & -43.48 \\
\hline 104.4 & $13: 10$ & 0.868 & -21.4 & -25.1 & .23 .9 & .44 .0 & -43.8 & -45.2 & .54 .3 & -52.6 & -92.3 & -62.5 & .73 .5 & -56.8 & & 8.8 & -11.1 & 30.8 & .40 .4 & -41.78 \\
\hline $121 \%$ & $13: 20$ & -0.861 & -29.4 & -28.0 & -23.9 & .44 .8 & .44 .7 & -45.2 & -51.3 & .52 .6 & -92.3 & -62.5 & .73 .5 & -56.9 & & 8.6 & -11.1 & 31.5 & -41.3 & -40.88 \\
\hline $121 \%$ & $13: 30$ & -0.854 & -22.3 & -25.1 & -23.9 & .45 .7 & -47.3 & -46.1 & -52.1 & -52.6 & .92 .3 & -62.5 & .77 .0 & .56 .9 & & 6.9 & -11.1 & 32.4 & -40.4 & -40.88 \\
\hline $1 \% 4,1$ & $13: 40$ & -0.847 & -22.3 & -26.8 & -24.8 & -48.6 & -46.4 & -48.9 & -52.1 & -53.5 & -92.3 & .62 .5 & .76 .1 & .58 .7 & & 0.8 & -11.1 & 30.6 & $\$ 1.3$ & -39.88 \\
\hline \% & $13: 50$ & -0.840 & -22.3 & -26.0 & -23.9 & .46 .6 & -47.3 & -46.9 & -52.1 & -54.4 & -82.3 & -62.5 & .77 .0 & .57 .8 & & 0.8 & -12.8 & 30.7 & .40 .4 & -40.88 \\
\hline 16 & $14: 00$ & -0.833 & -23.1 & -26.9 & -24.8 & .44 .8 & -47.3 & -46.9 & -52.1 & -55.3 & -92.3 & -62.5 & .76 .1 & .57 .8 & & 0.9 & -11.8 & 30.7 & -40.4 & -40.88 \\
\hline $12 \%$ & 14:10 & -0.826 & -23.1 & -26.9 & -24.8 & -46.8 & -47.3 & -40.1 & .52 .1 & -54.4 & -92.3 & -62.5 & .78 .1 & -56.8 & & 6.9 & .11 .1 & 30.7 & -42.1 & -40.86 \\
\hline (1) 6 & $14: 20$ & -0.019 & -22.3 & -27.7 & .25 .7 & .46 .6 & .47 .3 & -46.9 & -53.0 & -54.4 & -92.3 & -62.5 & .75 .2 & .58 .7 & & 6.9 & .13 .6 & 30.7 & -42.1 & -40.88 \\
\hline 6.1, & $14: 30$ & -0.812 & -24.0 & -27.7 & -28.5 & .45 .7 & .46 .4 & -46.1 & -52.1 & -56.2 & .92 .3 & .63 .4 & .78 .1 & .58 .7 & & 8.1 & .12 .8 & 30.7 & .43 .0 & -40.88 \\
\hline $12, \%$ & $14: 40$ & -0.806 & -23.1 & -27.7 & -28.5 & .47 .4 & .47 .3 & -46.9 & -52.1 & -55.3 & -94.1 & -63.4 & .77 .0 & -58.7 & & 8.8 & .12 .8 & 30.7 & -43.0 & -40.88 \\
\hline $101 \%$ & 14.50 & 0.799 & -22.3 & -27.7 & -25.7 & .47 .4 & .47 .3 & -46.9 & -53.0 & -55.3 & .92 .3 & -65.1 & .77 .0 & -59.5 & & 8.9 & -12.8 & 30.7 & -43.8 & -42.58 \\
\hline 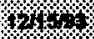 & $15 ; 0$ & -0.792 & -22.3 & -27.7 & -26.5 & -47.4 & -47.3 & -46.1 & -53.8 & -55.3 & .94 .9 & -86.0 & .78 .1 & -60.4 & & 8.9 & -13.6 & 28.1 & .43 .8 & $\begin{array}{r}-44.28 \\
\end{array}$ \\
\hline $421=$ & $15 ; 10$ & -0.786 & -22.3 & -27.7 & .25 .7 & .46 .6 & .46 .4 & -46.1 & -52.1 & -55.3 & .94 .8 & .65 .1 & .77 .0 & -60.4 & & 8.9 & -12.8 & 29.0 & 43.8 & .44 .28 \\
\hline $6 / 146$ & $15: 20$ & -0.778 & -23.1 & -27.7 & .25 .7 & -46.8 & -48.4 & -48.9 & -52.1 & -56.2 & .92 .3 & -64.2 & .78 .1 & .59 .5 & & 6.8 & -13.6 & 29.8 & -43.8 & -44.28 \\
\hline 121,4 & $15: 30$ & -0.771 & -22.3 & -27.7 & .26 .5 & .48 .8 & -47.3 & -46.1 & -52.1 & -55.3 & .92 .3 & -62.5 & .76 .1 & -58.7 & & 0.9 & -12.8 & 30.7 & -43.8 & -44.28 \\
\hline $12,1,33$ & $15: 40$ & -0.764 & -22.3 & -26.9 & .28 .5 & -46.6 & -48.4 & -46.1 & -52.1 & -55.3 & .93 .2 & -83.4 & .77 .0 & .58 .7 & & 6.8 & -12.8 & 30.7 & -44.7 & -44.28 \\
\hline$\%$ \% & $15: 50$ & -0.757 & -22.3 & -26.9 & .25 .7 & .46 .8 & -46.4 & -46.1 & .52 .1 & -55.3 & .94 .1 & -62.5 & .76 .1 & -59.5 & & 6.9 & -13.6 & 30.7 & -43.8 & .44 .28 \\
\hline $1 \% \%$ & $16: 00$ & -0.750 & -24.0 & -28.9 & .25 .7 & -46.8 & -46.4 & -46.1 & .52 .1 & -55.3 & .92 .3 & -62.5 & .77 .0 & -80.4 & & 0.8 & -13.6 & 30.7 & 43.8 & -44.28 \\
\hline $121 \%$ & 16:10 & -0.743 & -23.1 & -27.7 & .28 .5 & .47 .4 & .46 .4 & -46.9 & .53 .0 & -55.3 & .94 .1 & .63 .4 & .77 .0 & -58.7 & & 6.8 & -12.8 & 30.7 & 43.8 & 44.28 \\
\hline $6 \%$ & $16: 40$ & -0.722 & -24.0 & -28.6 & -28.5 & .47 .4 & -46.4 & -46.9 & .53 .0 & -54.4 & .93 .2 & .62 .5 & .77 .0 & -59.5 & & 6.8 & -13.6 & 30.7 & -43.8 & -44.28 \\
\hline \% & $16: 50$ & -0.716 & -24.0 & -27.7 & -26.5 & .46 .6 & -46.4 & -46.0 & -52.1 & -55.3 & .94 .1 & -63.4 & .76 .1 & -59.5 & & 8.9 & -12.7 & 30.7 & -43.8 & -44.28 \\
\hline 1410 & $17: 00$ & 0.708 & -23.1 & -27.7 & -28.5 & -46.8 & .47 .3 & -46.9 & .52 .1 & -55.3 & $\begin{array}{l}.94 .1 \\
\end{array}$ & -62.5 & .78 .1 & -59.5 & & 7.0 & -12.7 & 30.7 & 43.8 & -44.28 \\
\hline 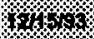 & 17:10 & -0.701 & -23.1 & -28.6 & -28.5 & -47.4 & .47 .3 & -46.9 & -52.1 & -55.3 & .94 .1 & -63.4 & .77 .0 & -59.5 & & 7.0 & -12.7 & 30.7 & 44.7 & -45.08 \\
\hline 1. & $17: 20$ & 0.694 & -23.1 & -28.6 & -27.4 & .47 .4 & -46.4 & -46.9 & -53.0 & -55.3 & -94.1 & -84.2 & -77.0 & -59.5 & & 7.0 & -13.6 & 30.7 & .43 .8 & -45.88 \\
\hline 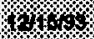 & $17: 30$ & -0.687 & -24.0 & -27.7 & -28.5 & -48.3 & .46 .4 & -46.9 & -53.0 & -55.3 & .94 .1 & -63.4 & -76.1 & .59 .5 & & 7.0 & -12.7 & 29.8 & -44.7 & -45.98 \\
\hline 621.4 & $17: 40$ & -0.681 & .23 .1 & -27.7 & -28.5 & -47.4 & .47 .3 & -46.0 & -52.1 & -55.3 & -94.1 & -83.4 & $\begin{array}{l}.76 .9 \\
\end{array}$ & -58.7 & & 7.0 & -12.7 & 30.7 & -43.8 & -45.88 \\
\hline 1210 & $17: 50$ & -0.674 & -24.9 & -27.7 & -28.5 & -46.6 & .46 .4 & -46.9 & -53.8 & -54.4 & -94.8 & -62.5 & -76.1 & .58 .7 & & 7.0 & -12.7 & 30.7 & -45.5 & -46.78 \\
\hline $2 \%$ & $18: 00$ & -0.867 & .23 .1 & -27.7 & -28.5 & -47.4 & .46 .4 & -46.9 & .52 .1 & -55.3 & -94.1 & -62.5 & .76 .1 & .58 .7 & & 7.0 & -12.7 & 30.8 & -44.7 & -47.68 \\
\hline $624-1$ & 18:10 & -0.600 & -24.0 & -28.6 & -28.5 & -47.4 & -47.3 & -46.9 & .54 .7 & 55.3 & -94.9 & -63.4 & .76 .1 & -58.7 & & 7.0 & -12.7 & 30.8 & -45.5 & -47.68 \\
\hline 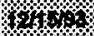 & $18: 20$ & 0.653 & .22 .3 & -27.7 & -26.5 & -47.4 & -48.1 & -47.8 & -53.8 & -55.3 & -94.1 & -63.4 & .76 .1 & -58.7 & & 7.0 & -12.7 & 30.8 & -46.4 & $\$ 7.68$ \\
\hline $4 x$ & 18:30 & -0.646 & -24.0 & -27.7 & -28.5 & -49.2 & -46.4 & -46.9 & -54.7 & -55.3 & .94 .9 & -63.4 & -78.1 & -60.4 & & 7.0 & .13 .6 & 29.8 & .45 .5 & -47.68 \\
\hline 1010 & $18: 40$ & -0.639 & -24.0 & -28.6 & -28.5 & -48.3 & -47.3 & -47.8 & -54.7 & -55.3 & .94 .8 & -63.4 & .70 .9 & 60.4 & & 7.0 & .13 .6 & 30.8 & -46.4 & -47.68 \\
\hline 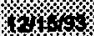 & $18: 50$ & -0.632 & -24.0 & -28.6 & .28 .5 & -47.4 & -47.3 & .47 .8 & .53 .8 & .55 .3 & .84 .8 & -83.4 & .76 .9 & -60.4 & & 6.1 & .13 .6 & 29.8 & -45.5 & -48.48 \\
\hline 12161 & $19: 00$ & -0.625 & -23.1 & -27.7 & .28 .5 & -48.3 & -47.3 & -48.7 & -53.0 & -55.3 & .04 .9 & .03 .4 & .77 .8 & -59.5 & & 0.1 & -13.6 & 30.8 & 46.4 & 48.46 \\
\hline $127.1 \%$ & \begin{tabular}{|l|}
19.10 \\
\end{tabular} & 0.618 & -24.0 & -28.6 & .28 .5 & -48.3 & -47.2 & -48.7 & .53 .8 & -55.3 & .85 .8 & .63 .3 & -77.8 & -59.5 & & 7.0 & -13.6 & 29.9 & -47.2 & -47.68 \\
\hline 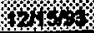 & $19: 20$ & -0.011 & -24.0 & -28.6 & .25 .7 & 47.4 & -47.2 & .48 .7 & .53 .8 & .55 .3 & .84 .8 & -63.3 & .78 .9 & $-\infty .4$ & & 0.1 & -13.5 & 28.9 & 47.2 & -48.48 \\
\hline
\end{tabular}


Table 5. Tensiometer Data

\begin{tabular}{|c|c|c|c|c|c|c|c|c|c|c|c|c|c|c|c|c|c|c|c|c|}
\hline & & & & & & & & & Ten: & slometer : & Data & & & & & & & & & \\
\hline & & Test & $\begin{array}{c}\text { Soil } \\
\text { Moisture } \\
\text { Tension } \\
\left(\mathrm{Cm} \mathrm{H}_{2} \mathrm{O}\right) \\
\end{array}$ & $\begin{array}{c}\text { Soll } \\
\text { Molsture } \\
\text { Tension } \\
\left(\mathrm{Cm} \mathrm{H}_{2} \mathrm{O}\right) \\
\end{array}$ & $\begin{array}{c}\text { Soil } \\
\text { Molsture } \\
\text { Tension } \\
\left(\mathrm{Cm} \mathrm{H}_{2} \mathrm{O}\right) \\
\end{array}$ & $\begin{array}{c}\text { Soil } \\
\text { Molsture } \\
\text { Tension } \\
\left(\mathrm{cm} \mathrm{H}_{2} \mathrm{O}\right) \\
\end{array}$ & $\begin{array}{c}\text { Soll } \\
\text { Molsture } \\
\text { Tension } \\
\left(\mathrm{cm} \mathrm{H}_{2} \mathrm{O}\right) \\
\end{array}$ & $\begin{array}{c}\text { Soil } \\
\text { Moisture } \\
\text { Tension } \\
\left(\mathrm{em} \mathrm{H}_{2} \mathrm{O}\right) \\
\end{array}$ & $\begin{array}{c}\text { Soll } \\
\text { Moisture } \\
\text { Tension } \\
\left(\mathrm{Cm} \mathrm{H}_{2} \mathrm{O}\right) \\
\end{array}$ & $\begin{array}{c}\text { Soll } \\
\text { Molsture } \\
\text { Tension } \\
\left(\mathrm{cm} \mathrm{H}_{2} \mathrm{O}\right) \\
\end{array}$ & $\begin{array}{c}\text { Soil } \\
\text { Moisture } \\
\text { Tansion } \\
\left(\mathrm{cm} \mathrm{H}_{2} \mathrm{O}\right) \\
\end{array}$ & $\begin{array}{c}\text { Soll } \\
\text { Moisture } \\
\text { Tension } \\
\left(\mathrm{Cm} \mathrm{H}_{2} \mathrm{O}\right) \\
\end{array}$ & $\begin{array}{c}\text { Soll } \\
\text { Molsture } \\
\text { Tension } \\
\left(\mathrm{Cm} \mathrm{H}_{2} \mathrm{O}\right) \\
\end{array}$ & $\begin{array}{c}\text { Soll } \\
\text { Moisture } \\
\text { Tension } \\
\left(\mathrm{Cm}^{\mathrm{H}} \mathrm{O}\right) \\
\end{array}$ & $\begin{array}{c}\text { Soll } \\
\text { Moisture } \\
\text { Tension } \\
\left(\mathrm{Cm} \mathrm{H}_{2} \mathrm{O}\right) \\
\end{array}$ & $\begin{array}{c}\text { Soll } \\
\text { Molsture } \\
\text { Tension } \\
\left(\mathrm{Cm} \mathrm{H}_{2} \mathrm{O}\right) \\
\end{array}$ & $\begin{array}{c}\text { Soll } \\
\text { Molsture } \\
\text { Tenslon } \\
\left.\text { (cm } \mathrm{H}_{2} \mathrm{O}\right) \\
\end{array}$ & $\begin{array}{c}\text { Soll } \\
\text { Molsture } \\
\text { Tension } \\
\left(\mathrm{Cm} \mathrm{H}_{2} \mathrm{O}\right) \\
\end{array}$ & 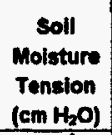 & $\begin{array}{c}\text { Soll } \\
\text { Molsture } \\
\text { Tension } \\
\left(\mathrm{Cm} \mathrm{H}_{2} \mathrm{O}\right) \\
\end{array}$ \\
\hline Date & Time & Days' & $C 1^{a}$ & $B 1^{a}$ & $A 1^{a}$ & $\mathrm{C2}^{\mathrm{b}}$ & $B 2^{6}$ & $A 2^{b}$ & $\mathrm{C3}^{\mathrm{C}}$ & $\mathrm{B}^{\mathrm{C}}$ & $A 3^{\mathrm{C}}$ & $C 4^{d}$ & $B 4^{\mathrm{d}}$ & $A 4^{d}$ & $C 5^{\circ}$ & $B 5^{\circ}$ & $A 5^{\circ}$ & $\mathrm{Cb}^{\mathrm{f}}$ & $B 6^{\prime}$ & $A G^{\prime}$ \\
\hline 16\% & $19: 30$ & -0.004 & -24.0 & -28.6 & -28.3 & -47.4 & -47.2 & -48.7 & -54.7 & -55.3 & -94.9 & -62.5 & -78.9 & -58.7 & & 0.1 & -13.5 & 29.9 & -48.3 & -48.48 \\
\hline $14 \%$ & $19: 40$ & -0.697 & -24.0 & -28.8 & -28.5 & -47.4 & -47.2 & -46.9 & -53.8 & -55.3 & .94 .9 & -62.5 & -76.9 & -60.4 & & 6.2 & -13.5 & 29.9 & -46.3 & -49.38 \\
\hline 124.6 & $19: 50$ & 0.690 & -23.1 & -27.7 & -27.4 & -46.6 & -47.2 & -46.0 & -53.8 & -55.3 & -94.9 & -65.1 & -77.8 & -59.5 & & 0.2 & -13.5 & 29.9 & -46.3 & -50.18 \\
\hline 12164 & $20: 00$ & -0.583 & -22.3 & -29.5 & -28.5 & -47.4 & -47.2 & -47.8 & -53.8 & -55.3 & .94 .9 & -64.2 & .76 .9 & .59 .5 & & 4.4 & -13.5 & 30.0 & -46.3 & -49.38 \\
\hline$\% 24 \%$ & $20: 10$ & -0.676 & -23.1 & -28.6 & -26.5 & -47.4 & -46.4 & -46.0 & -54.7 & -55.3 & -94.9 & -64.2 & -76.9 & -60.4 & & 8.2 & -13.5 & 30.0 & -47.2 & -49.36 \\
\hline $1 \% 4$ & $20: 20$ & -0.569 & -23.1 & -28.6 & -27.4 & -47.4 & -47.2 & -48.8 & -54.7 & -55.3 & -94.9 & -65.1 & .77 .8 & .60 .4 & & 6.2 & -12.7 & 30.0 & -47.2 & -50.18 \\
\hline 22144 & $20: 30$ & -0.662 & -24.0 & -28.6 & -26.5 & -48.3 & -47.2 & -46.9 & -53.0 & -55.3 & -94.9 & -64.2 & -76.9 & -60.4 & & 8.2 & -11.8 & 30.0 & .47 .2 & -51.08 \\
\hline 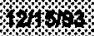 & $20: 40$ & -0.556 & -24.0 & -28.6 & -27.4 & -47.4 & -48.1 & -47.8 & -53.8 & -55.3 & .94 .9 & -65.1 & .77 .8 & .60 .4 & & 6.2 & -12.7 & 29.1 & -47.2 & -50.18 \\
\hline$/ 11 \%$ & $20: 50$ & 0.649 & .24 .0 & .28 .6 & -26.5 & -47.4 & -48.1 & -47.8 & -53.8 & -55.3 & -94.9 & -64.2 & .78 .9 & -60.4 & & 5.3 & -13.5 & 30.0 & -47.2 & -49.28 \\
\hline $4 \%$ & $21: 00$ & -0.642 & -24.0 & -28.6 & -27.4 & -47.4 & -47.2 & -47.8 & -53.8 & .56 .1 & .94 .1 & -64.2 & .78 .9 & -59.5 & & 8.2 & -13.5 & 30.0 & -47.2 & -50.18 \\
\hline Yyor & $21: 10$ & 0.635 & -24.0 & -28.6 & -27.4 & -49.2 & -47.2 & -46.9 & -53.8 & -55.3 & -94.9 & -65.1 & .77 .8 & -60.4 & & 5.3 & -11.8 & 29.1 & -48.1 & .51 .88 \\
\hline $12.16 \%$ & $21: 20$ & -0.628 & -23.1 & -28.6 & -27.4 & -47.4 & -48.1 & -48.7 & -52.1 & .55 .3 & .94 .9 & -64.2 & .76 .9 & -60.4 & & 7.1 & -12.6 & 30.0 & -48.1 & -51.08 \\
\hline $12106 \%$ & 21:30 & -0.521 & -24.0 & -28.6 & -27.4 & -47.4 & -47.2 & -46.0 & -52.9 & -54.4 & -85.8 & -64.2 & -77.8 & -59.5 & & 4.5 & -11.8 & 30.0 & .48 .9 & .51 .88 \\
\hline 11119 & $21: 40$ & -0.514 & -23.1 & -28.6 & -27.4 & -47.4 & -47.2 & -46.9 & .52 .8 & -55.3 & .85 .8 & -64.2 & -78.9 & -59.5 & & 6.2 & -12.8 & 30.0 & .47 .2 & -51.88 \\
\hline 4224.9 & $21: 50$ & 0.607 & -23.1 & -28.6 & -27.4 & -47.4 & -47.2 & -46.9 & -52.1 & -56.1 & -95.8 & -64.2 & .78 .9 & -60.4 & & 8.2 & -11.8 & 30.0 & -48.0 & -51.88 \\
\hline $1516 \%$ & $22: 00$ & -0.600 & -24.0 & -28.6 & -27.4 & -47.4 & -48.1 & -46.9 & .52 .8 & -55.3 & -94.9 & -64.2 & -78.9 & -58.5 & & 6.2 & -12.8 & 30.0 & -48.0 & .51 .08 \\
\hline 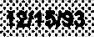 & $22: 10$ & -0.493 & -24.0 & -28.6 & -26.5 & -47.4 & -47.2 & -46.0 & -52.9 & -55.2 & -95.8 & -65.1 & -77.8 & -59.5 & & 5.3 & -12.6 & 30.0 & -49.8 & -51.88 \\
\hline 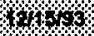 & $22: 20$ & -0.486 & -24.0 & -28.6 & -27.4 & -47.4 & -47.2 & -46.0 & .52 .9 & -55.3 & -95.8 & -63.3 & -76.9 & -59.5 & & 6.2 & -11.8 & 29.1 & -48.8 & -51.88 \\
\hline 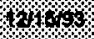 & $22: 30$ & 0.479 & -24.0 & -28.6 & .27 .4 & -47.4 & -47.2 & -48.0 & -52.9 & -54.4 & -95.8 & -64.2 & -78.9 & -59.5 & & 5.3 & -13.5 & 30.0 & -48.0 & -52.78 \\
\hline 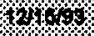 & $22: 40$ & -0.472 & -24.0 & -28.6 & -27.4 & -47.4 & -47.2 & -46.8 & -53.8 & -55.2 & -95.8 & -63.3 & -76.9 & -60.4 & & 5.3 & -12.6 & 30.0 & -48.0 & .52 .78 \\
\hline $14 \%$ & $22: 50$ & 0.465 & -24.0 & -28.6 & -27.4 & -47.4 & -47.2 & -46.9 & -53.8 & -55.2 & -95.8 & -63.3 & -77.8 & -60.4 & & 6.2 & -12.6 & 28.3 & -48.0 & -53.58 \\
\hline 8140,6 & $23: 00$ & -0.458 & -24.0 & -28.6 & -27.4 & -47.4 & -47.2 & 46.9 & -53.8 & -55.2 & -95.8 & -63.3 & -76.9 & -58.5 & & 5.3 & -11.8 & 30.0 & .50 .6 & .52 .78 \\
\hline 821040 & $23: 10$ & 0.451 & -24.0 & -27.7 & -27.4 & -47.4 & -47.2 & -46.9 & -53.8 & -55.2 & -95.8 & -63.3 & -78.9 & -59.5 & & 6.2 & -11.8 & 30.0 & -48.9 & -53.58 \\
\hline $1 / 40 \%$ & $23: 20$ & 0.444 & -24.0 & -28.6 & -27.4 & -47.4 & -47.2 & -47.8 & .53 .8 & -54.4 & -95.8 & .66 .0 & .78 .9 & -60.4 & & 7.1 & -11.8 & 30.0 & -48.8 & .53 .58 \\
\hline 146 & 23:30 & -0.437 & -24.0 & -28.6 & -28.3 & -47.4 & -47.2 & -48.9 & -52.9 & -55.2 & .94 .9 & -64.2 & .77 .8 & -59.5 & & 5.4 & -11.8 & 30.9 & 48.0 & -53.58 \\
\hline 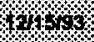 & $23: 40$ & 0.431 & -24.0 & -28.6 & -27.4 & -47.4 & .47 .2 & -46.9 & .53 .8 & .55 .2 & .95 .8 & .64 .2 & .77 .8 & -60.4 & & 6.2 & -11.7 & 30.0 & .48 .9 & -54.48 \\
\hline 1014 & $23: 50$ & -0.424 & -24.0 & -28.8 & -27.4 & -47.4 & -48.1 & .47 .8 & .52 .9 & -55.2 & .94 .8 & .64 .2 & .76 .9 & .59 .5 & & 4.5 & .12 .6 & 30.0 & -49.8 & -54.48 \\
\hline 4216. & $0: 00$ & -0.417 & -24.0 & -28.6 & -27.4 & -47.4 & .48 .1 & -46.9 & .53 .8 & -56.1 & -95.8 & .64 .2 & .77 .8 & .59 .5 & & 5.4 & .12 .6 & 29.2 & -49.8 & -54.48 \\
\hline 184169 & $0: 10$ & -0.410 & .23 .1 & .28 .6 & -27.4 & -47.4 & -48.1 & -46.0 & -54.7 & -56.1 & .95 .8 & -64.2 & .77 .8 & -59.5 & & 5.4 & .13 .5 & 29.2 & -48.8 & -55.28 \\
\hline $171016 \%$ & 0.20 & -0.403 & .24 .0 & -28.6 & -28.5 & -48.3 & -47.2 & -46.9 & -52.9 & -55.2 & .95 .8 & -63.3 & .76 .9 & -60.4 & & 4.5 & -12.6 & 30.1 & .50 .6 & -56.18 \\
\hline $1 \% 4 \%$ & $0: 30$ & -0.396 & .24 .0 & -28.6 & .27 .4 & .47 .4 & -48.1 & -46.8 & .52 .8 & -55.2 & -95.8 & -64.2 & -76.9 & -59.5 & & 5.4 & -11.7 & 30.1 & .50 .6 & -56.18 \\
\hline /15\% & $0: 40$ & 0.389 & .24 .0 & -29.5 & -27.4 & -47.4 & -48.0 & -47.8 & .52 .9 & .56 .1 & $-95.8^{\circ}$ & -64.2 & .78 .9 & 60.4 & & 5.4 & -11.7 & 29.2 & -50.6 & -55.28 \\
\hline $121.6 \%$ & 0.50 & 0.382 & .24 .0 & .28 .6 & -27.4 & -47.4 & -48.1 & -47.8 & .53 .8 & .55 .2 & -95.8 & -64.2 & .78 .6 & -58.5 & & 5.4 & -13.5 & 29.2 & -50.6 & -56.18 \\
\hline 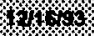 & $1: 00$ & -0.375 & .24 .0 & .28 .6 & -27.4 & -47.4 & -47.2 & -46.8 & -52.9 & .55 .2 & -95.8 & -64.2 & .77 .8 & 60.4 & & 5.4 & -13.5 & 30.1 & -50.6 & -57.88 \\
\hline $111 \%$ & 1:10 & -0.368 & -24.0 & .28 .6 & .27 .4 & -47.4 & -49.0 & .46 .9 & .54 .7 & -57.0 & -95.8 & -65.9 & -76.9 & -59.5 & & 4.5 & -13.5 & 30.1 & -50.6 & -57.88 \\
\hline $10 \% 161$ & 1:20 & -0.361 & -24.0 & .28 .6 & -27.4 & -47.4 & -47.2 & .46 .8 & .53 .8 & -55.2 & -95.8 & -65.9 & .76 .9 & -59.5 & & 4.5 & -13.5 & 30.1 & -50.6 & -57.88 \\
\hline $81410 \%$ & $1: 30$ & -0.364 & .24 .0 & -28.6 & -27.4 & -47.4 & .48 .1 & .46 .8 & .52 .8 & -56.1 & -94.8 & .64 .2 & .78 .9 & 60.4 & & 4.5 & -12.6 & 30.1 & -50.8 & .57 .88 \\
\hline 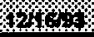 & $1: 40$ & 0.347 & .24 .0 & .28 .6 & -27.4 & -47.4 & -47.2 & .46 .9 & .53 .8 & .56 .1 & -95.8 & -64.2 & -76.0 & -59.5 & & 4.5 & .13 .5 & 30.1 & 50.6 & -59.58 \\
\hline
\end{tabular}


Table 5. Tensiometer Data

\begin{tabular}{|c|c|c|c|c|c|c|c|c|c|c|c|c|c|c|c|c|c|c|c|c|}
\hline & & & & & & & & & Ten: & siometer & Data & & & & & & & & & \\
\hline & & Test & $\begin{array}{c}\text { Soll } \\
\text { Molisture } \\
\text { Tension } \\
\left(\mathrm{Cm} \mathrm{H}_{2} \mathrm{O}\right)\end{array}$ & $\begin{array}{c}\text { Soll } \\
\text { Moisture } \\
\text { Tenston } \\
\left(\mathrm{Cm} \mathrm{H}_{2} \mathrm{O}\right)\end{array}$ & $\begin{array}{c}\text { Soll } \\
\text { Molsture } \\
\text { Tenslon } \\
\left(\mathrm{Cm} \mathrm{H}_{2} \mathrm{O}\right) \\
\end{array}$ & $\begin{array}{c}\text { Soll } \\
\text { Moisture } \\
\text { Tension } \\
\left(\mathrm{Cm}_{2} \mathrm{O}\right) \\
\end{array}$ & $\begin{array}{c}\text { Soll } \\
\text { Moisture } \\
\text { Tension } \\
\left(\mathrm{cm} \mathrm{H}_{2} \mathrm{O}\right) \\
\end{array}$ & $\begin{array}{c}\text { Soll } \\
\text { Moisture } \\
\text { Tension } \\
\left(\mathrm{Cm} \mathrm{H}_{2} \mathrm{O}\right)\end{array}$ & $\begin{array}{c}\text { Soll } \\
\text { Moisture } \\
\text { Tension } \\
\left(\mathrm{Cm} \mathrm{H}_{2} \mathrm{O}\right) \\
\end{array}$ & 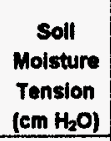 & $\begin{array}{c}\text { Soll } \\
\text { Moisture } \\
\text { Tension } \\
\left(\mathrm{Cm} \mathrm{H}_{2} \mathrm{O}\right) \\
\end{array}$ & $\begin{array}{c}\text { Soll } \\
\text { Moisture } \\
\text { Tenslon } \\
\left(\mathrm{em} \mathrm{H}_{2} \mathrm{O}\right) \\
\end{array}$ & $\begin{array}{c}\text { Soll } \\
\text { Molsture } \\
\text { Tension } \\
\left.\text { (cm } \mathrm{H}_{2} \mathrm{O}\right) \\
\end{array}$ & $\begin{array}{c}\text { Soll } \\
\text { Moisture } \\
\text { Tension } \\
\left(\mathrm{cm} \mathrm{H}_{2} \mathrm{O}\right) \\
\end{array}$ & $\begin{array}{c}\text { Soll } \\
\text { Molsture } \\
\text { Tension } \\
\left(\mathrm{Cm} \mathrm{H}_{2} \mathrm{O}\right)\end{array}$ & 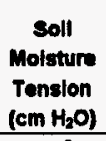 & $\begin{array}{c}\text { Soll } \\
\text { Molsture } \\
\text { Tension } \\
\left(\mathrm{Cm} \mathrm{H}_{2} \mathrm{O}\right)\end{array}$ & $\begin{array}{c}\text { Soll } \\
\text { Moisture } \\
\text { Tension } \\
\left(\mathrm{Cm} \mathrm{H}_{2} \mathrm{O}\right) \\
\end{array}$ & $\begin{array}{c}\text { Soll } \\
\text { Molsture } \\
\text { Tension } \\
\left(\mathrm{Cm} \mathrm{H}_{2} \mathrm{O}\right)\end{array}$ & $\begin{array}{c}\text { Soll } \\
\text { Molsturs } \\
\text { Tenalon } \\
\left(\mathrm{Cm} \mathrm{H}_{2} \mathrm{O}\right) \\
\end{array}$ \\
\hline Date & Time & Days' & $C 1^{\prime \prime}$ & $B 1^{*}$ & $A 1^{\prime \prime}$ & $\mathrm{C2}^{\mathrm{b}}$ & $B 2^{\circ}$ & $A 2^{b}$ & $\mathrm{C3}^{\mathrm{C}}$ & $\mathrm{B3}^{\mathrm{C}}$ & $A 3^{c}$ & $\mathrm{CA}^{\mathrm{d}}$ & $B 4^{d}$ & $\mathrm{Aq}^{\mathrm{d}}$ & $65^{\circ}$ & $B 6^{\circ}$ & $A 5^{\circ}$ & $C B^{f}$ & $B 6^{\prime}$ & $A 6^{\prime}$ \\
\hline Yor & $1: 50$ & -0.340 & -24.0 & -28.6 & -28.5 & -47.4 & -48.1 & -46.8 & .54 .7 & .56 .1 & -95.8 & -65.1 & -78.9 & -58.6 & & 5.4 & .12 .8 & 30.1 & .50 .8 & -57.88 \\
\hline (2) & $2: 00$ & -0.333 & -24.0 & -28.6 & -27.4 & -47.4 & -47.2 & -47.8 & .53 .8 & .55 .2 & -95.8 & -65.9 & .77 .8 & -80.4 & & 4.5 & -12.6 & 29.2 & .50 .6 & -57.80 \\
\hline \% & $2: 10$ & -0.326 & -24.0 & -28.6 & -27.4 & -47.4 & -47.2 & -46.8 & -52.9 & .58 .1 & -95.8 & -64.2 & .78 .9 & -59.5 & & 5.4 & .13 .5 & 30.1 & -51.5 & -57.88 \\
\hline 124,69 & $2: 30$ & -0.319 & -23.1 & -28.6 & -27.4 & -47.4 & .47 .2 & .46 .8 & -53.8 & .58 .1 & -85.8 & .65 .9 & .78 .6 & .59 .5 & & 7.1 & .13 .5 & 28.3 & .50 .6 & -56.98 \\
\hline 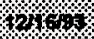 & $2: 30$ & -0.312 & -24.0 & -28.6 & -27.4 & -47.4 & -47.2 & -47.8 & .53 .8 & .56 .1 & -95.8 & -65.9 & .77 .8 & -60.4 & & 6.3 & -11.7 & 30.1 & .50 .8 & -58.78 \\
\hline $81616 \%$ & $2: 40$ & 0.300 & -23.1 & -28.6 & -26.5 & -47.4 & -47.2 & .46 .8 & .52 .9 & .55 .2 & -95.8 & -65.9 & -77.8 & -60.4 & & 4.5 & .13 .5 & 30.1 & .50 .6 & -59.58 \\
\hline $821 \%$ & $2: 50$ & -0.289 & -24.0 & -28.6 & -26.5 & -47.4 & .47 .2 & -46.9 & .53 .8 & .58 .1 & -95.8 & -65.1 & .78 .8 & -60.4 & & 5.4 & .13 .5 & 30.1 & .50 .6 & -58.78 \\
\hline 4246 & $3: 00$ & -0.292 & -24.0 & -28.6 & -27.4 & -47.4 & -47.2 & -46.9 & .53 .8 & .56 .1 & -95.8 & -65.1 & .76 .9 & -60.4 & & 5.4 & -13.4 & 30.1 & .50 .6 & .59 .58 \\
\hline 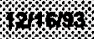 & $3: 10$ & -0.285 & -24.0 & -28.5 & -27.4 & -47.4 & .49 .0 & -47.8 & .53 .8 & .56 .1 & .95 .8 & -65.9 & .77 .8 & -60.4 & & 4.5 & $\begin{array}{r}-12.6 \\
\end{array}$ & 30.1 & .50 .6 & -59.58 \\
\hline 1010. & $3: 20$ & -0.278 & -24.0 & -28.6 & -27.4 & -48.3 & .48 .1 & -46.8 & .54 .7 & .56 .1 & -95.8 & -65.1 & .77 .8 & -80.4 & & 8.3 & .12 .6 & 30.1 & .51 .5 & .59 .58 \\
\hline 5464 & $3: 30$ & -0.271 & -24.0 & -28.6 & -27.4 & -47.4 & .48 .1 & .46 .9 & -53.8 & .54 .4 & .95 .8 & -65.1 & .76 .8 & .60 .4 & & 4.5 & -11.7 & 30.1 & .50 .8 & -60.48 \\
\hline 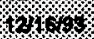 & 3:40 & -0.264 & -24.0 & -29.5 & -27.4 & -47.4 & .48 .1 & .46 .8 & -54.7 & .56 .1 & -85.8 & -64.2 & -76.8 & -60.4 & & 5.4 & .13 .4 & 30.1 & .51 .5 & .59 .58 \\
\hline 8721604 & $3: 50$ & -0.25 & -24.0 & -28.6 & -27.4 & .47 .4 & .49 .0 & -48.7 & .53 .8 & -57.0 & .95 .8 & -65.1 & .76 .8 & -59.5 & & 4.5 & -11.7 & 30.1 & .51 .5 & .59 .58 \\
\hline 42.64 & $4: 00$ & -0.250 & -24.0 & -26.9 & -27.4 & -47.4 & -48.1 & -46.9 & .54 .7 & -58.1 & -95.8 & -65.9 & -77.8 & 60.4 & & 4.5 & .12 .8 & 28.3 & .50 .6 & -81.28 \\
\hline $4 \%$ & $4: 10$ & 0.213 & -24.0 & -28.6 & .27 .4 & .47 .4 & .47 .2 & .47 .8 & .53 .8 & .58 .1 & -95.8 & -65.1 & .77 .8 & -59.5 & & 4.5 & -13.4 & 29.2 & .52 .3 & -61.28 \\
\hline 42161 & $4: 20$ & -0.236 & -24.0 & -28.6 & .27 .4 & -47.4 & .47 .2 & -46.9 & .54 .7 & .58 .1 & -95.8 & -65.9 & .76 .9 & -59.5 & & 4.5 & -10.8 & 29.2 & -51.5 & -61.28 \\
\hline $12,4 \times$ & $4: 30$ & -0.229 & -24.0 & -28.6 & .27 .4 & .47 .4 & .47 .2 & -46.8 & .54 .7 & .55 .2 & -95.8 & -65.9 & .77 .8 & -59.5 & & 3.7 & -10.0 & 27.5 & .53 .2 & -81.28 \\
\hline 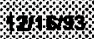 & $4: 40$ & -0.222 & -24.0 & -28.6 & -27.4 & .47 .4 & -47.2 & -46.0 & .52 .9 & .56 .1 & -95.8 & -65.8 & -78.6 & .58 .6 & & 4.5 & -10.8 & 30.1 & .52 .3 & -62.18 \\
\hline (3) & $4: 50$ & 0.215 & -24.0 & -28.6 & .27 .4 & .47 .4 & .47 .2 & .46 .9 & .53 .8 & .58 .1 & -85.8 & -65.9 & .76 .9 & -57.7 & & 4.5 & -10.8 & 29.2 & .53 .2 & -02.18 \\
\hline 84216 & $5: 00$ & -0.208 & -24.0 & -28.6 & -27.4 & .47 .4 & .47 .2 & .46 .8 & .53 .8 & -56.1 & -85.8 & -65.9 & .78 .8 & -57.7 & & 5.4 & -10.8 & 30.1 & .53 .2 & -61.28 \\
\hline 4231620 & $5: 10$ & -0.201 & -24.0 & -28.6 & .27 .4 & .47 .4 & .47 .2 & -46.0 & .52 .9 & .58 .1 & .85 .8 & -65.9 & .77 .8 & -58.8 & & 4.5 & -10.8 & 29.2 & .53 .2 & -62.18 \\
\hline (3) & $5: 20$ & -0.194 & -24.0 & -28.6 & .27 .4 & .48 .3 & .47 .2 & -46.9 & .54 .7 & -56.1 & -95.8 & -65.1 & -76.9 & -56.9 & & 5.4 & -10.8 & 29.2 & .53 .2 & -81.28 \\
\hline 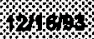 & $5: 30$ & -0.187 & -24.0 & -28.6 & .27 .4 & .47 .4 & -47.2 & -46.8 & .53 .8 & .55 .2 & .95 .8 & -65.1 & .77 .8 & -57.7 & & 3.7 & -10.0 & 29.2 & -54.1 & -02.18 \\
\hline $67 \%$ & $5: 40$ & -0.181 & .24 .0 & -28.6 & -26.5 & .47 .4 & .47 .2 & -46.8 & .54 .7 & .58 .1 & .95 .8 & -65.9 & -76.9 & .57 .7 & & 3.7 & .10 .8 & 30.1 & .54 .1 & -61.28 \\
\hline 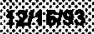 & $5: 50$ & -0.174 & -24.0 & -27.7 & -27.4 & .47 .4 & -47.2 & -46.8 & .54 .7 & .56 .1 & .95 .8 & .65 .1 & -77.8 & -57.7 & & 7.1 & -12.6 & 29.2 & .53 .2 & -62.18 \\
\hline$\%$ \% & $6: 00$ & -0.167 & -24.0 & -28.6 & -27.4 & -47.4 & .44 .7 & -46.8 & .54 .7 & .56 .1 & .95 .8 & .65 .9 & -78.6 & -57.7 & & 4.5 & .10 .8 & 28,4 & -52.3 & -63.08 \\
\hline $8130 \%$ & $6: 10$ & -0.100 & -24.0 & -28.6 & -27.4 & -47.4 & -47.2 & -46.8 & -53.8 & .57 .0 & .05 .8 & -65.9 & -77.8 & -56.8 & & 3.6 & -10.8 & 30.1 & -54.1 & -62.18 \\
\hline 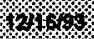 & $8: 20$ & -0.163 & -24.0 & -28.6 & -27.4 & -47.4 & -49.0 & -46.8 & -54.7 & .56 .1 & .05 .8 & .65 .8 & .77 .8 & -57.7 & & 4.5 & -11.7 & 29.2 & .54 .1 & .62 .88 \\
\hline $826 \%$ & $6: 30$ & -0.146 & .24 .8 & -28.6 & -27.4 & -47.4 & -47.2 & -46.0 & .54 .7 & .55 .2 & .95 .8 & -65.1 & .77 .8 & -57.7 & & 3.7 & -10.8 & 28.4 & -54.1 & -63.88 \\
\hline 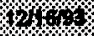 & $6: 40$ & -0.139 & .22 .2 & -27.7 & -27.4 & -48.3 & .47 .2 & .46 .8 & .54 .7 & .57 .0 & .86 .7 & -65.8 & .77 .8 & -57.7 & & 3.7 & -10.8 & 28.2 & -54.1 & -02.98 \\
\hline 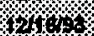 & $6: 50$ & 0.132 & .24 .0 & -28.6 & .27 .4 & -47.4 & -47.2 & -46.0 & -54.7 & .55 .2 & -95.8 & -65.1 & .77 .8 & .56 .9 & & 3.7 & -10.8 & 28.4 & -54.1 & -64.78 \\
\hline 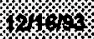 & $7: 00$ & -0.126 & -24.0 & -28.6 & .27 .4 & -48.3 & -47.2 & -46.9 & -54.7 & -56.1 & .85 .8 & .65 .8 & .77 .8 & .59 .5 & & 2.8 & -10.8 & 30.1 & .54 .1 & -64.78 \\
\hline 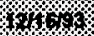 & $7: 10$ & 0.118 & .24 .0 & -29.5 & -27.4 & -47.4 & -47.2 & -46.9 & -54.7 & -56.1 & -95.8 & -65.9 & -77.8 & .57 .7 & & 3.7 & -10.8 & 28.4 & .54 .1 & -04.78 \\
\hline 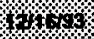 & $7: 20$ & 0.111 & .24 .0 & -29.5 & -27.4 & -47.4 & -49.0 & -47.8 & -53.8 & -56.1 & -85.8 & -65.1 & .76 .9 & .57 .7 & & 4.5 & -11.7 & 30.1 & .55 .8 & .64 .78 \\
\hline 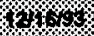 & $7: 30$ & -0.104 & .24 .8 & .28 .6 & -27.4 & -47.4 & -47.2 & -46.9 & -54.7 & -56.1 & -95.8 & -65.9 & .77 .8 & .58 .6 & & 4.5 & .90 .8 & 28.4 & .54 .1 & -65.58 \\
\hline 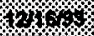 & $7: 40$ & -0.007 & -24.0 & -28.6 & .27 .4 & -47.4 & -47.2 & -46.9 & -54.7 & -56.1 & -05.8 & -65.9 & -77.8 & -59.5 & & 3.7 & -10.8 & 27.5 & -54.1 & 84.78 \\
\hline $125 \% 1$ & $7: 50$ & -0.000 & -24.0 & .28 .6 & .27 .4 & .47 .4 & -47.2 & -46.9 & -54.7 & -56.1 & -95.8 & -65.9 & -77.8 & .57 .7 & & 3.7 & -10.8 & 27.5 & .54 .1 & -65.58 \\
\hline 82460 & $8: 00$ & -0.083 & -24.0 & -28.6 & .27 .4 & .47 .4 & -47.2 & -46.0 & -54.7 & -56.1 & -95.8 & -65.9 & -77.8 & -57.7 & & 5.4 & -10.8 & 28.4 & -54.1 & .65 .58 \\
\hline
\end{tabular}


Table 5. Tensiometer Data

\begin{tabular}{|c|c|c|c|c|c|c|c|c|c|c|c|c|c|c|c|c|c|c|c|c|}
\hline & & & & & & & & & Ten & siometer & Data & & & & & & & & & \\
\hline & & Test & $\begin{array}{c}\text { Soll } \\
\text { Molsture } \\
\text { Tension } \\
\left(\mathrm{cm}_{2} \mathrm{O} \text { ) }\right. \\
\end{array}$ & $\begin{array}{c}\text { Soll } \\
\text { Molsture } \\
\text { Tension } \\
\left(\mathrm{cm} \mathrm{H}_{2} \mathrm{O}\right) \\
\end{array}$ & $\begin{array}{c}\text { Soll } \\
\text { Moisture } \\
\text { Tension } \\
\left(\mathrm{Cm} \mathrm{H}_{2} \mathrm{O}\right) \\
\end{array}$ & $\begin{array}{c}\text { Soll } \\
\text { Moisture } \\
\text { Tension } \\
\left(\mathrm{Cm} \mathrm{H}_{2} \mathrm{O}\right) \\
\end{array}$ & 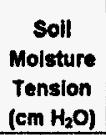 & $\begin{array}{c}\text { Soll } \\
\text { Moisture } \\
\text { Tension } \\
\left(\mathrm{Cm} \mathrm{H}_{2} \mathrm{O}\right)\end{array}$ & $\begin{array}{c}\text { Soll } \\
\text { Moisture } \\
\text { Tension } \\
\left(\mathrm{C} \mathrm{H}_{2} \mathrm{O}\right) \\
\end{array}$ & $\begin{array}{c}\text { Soil } \\
\text { Molsture } \\
\text { Tension } \\
\left(\mathrm{cm} \mathrm{H}_{2} \mathrm{O}\right) \\
\end{array}$ & 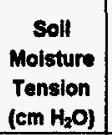 & $\begin{array}{c}\text { Soll } \\
\text { Moisture } \\
\text { Tension } \\
\left(\mathrm{C} \mathrm{H}_{2} \mathrm{O}\right) \\
\end{array}$ & $\begin{array}{l}\text { Soll } \\
\text { Moisture } \\
\text { Tension } \\
\left(\mathrm{Cm}^{\left.\mathrm{H}_{2} \mathrm{O}\right)}\right.\end{array}$ & $\begin{array}{c}\text { Soll } \\
\text { Moisture } \\
\text { Tenslon } \\
\left(\mathrm{Cm} \mathrm{H}_{2} \mathrm{O}\right) \\
\end{array}$ & $\begin{array}{c}\text { Soll } \\
\text { Moisture } \\
\text { Tension } \\
\left(\mathrm{cm} \mathrm{H}_{2} \mathrm{O}\right)\end{array}$ & $\begin{array}{c}\text { Soll } \\
\text { Moisture } \\
\text { Tension } \\
\left(\mathrm{cm} \mathrm{H} \mathrm{H}_{2} \mathrm{O}\right)\end{array}$ & 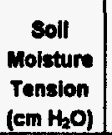 & $\begin{array}{c}\text { Soll } \\
\text { Moisture } \\
\text { Tenslon } \\
\left(\mathrm{Cm} \mathrm{H}_{2} \mathrm{O}\right)\end{array}$ & $\begin{array}{c}\text { Soll } \\
\text { Molsture } \\
\text { Tension } \\
\left(\mathrm{Cm} \mathrm{H}_{2} \mathrm{O}\right)\end{array}$ & $\begin{array}{c}\text { Soll } \\
\text { Molsture } \\
\text { Tension } \\
\left(\mathrm{em} \mathrm{H}_{2} \mathrm{O}\right)\end{array}$ \\
\hline Date & Time & Days ${ }^{*}$ & $\mathrm{C1}^{\prime \prime}$ & $B 1^{*}$ & $A 1^{\prime \prime}$ & $\mathrm{C2}^{\mathrm{b}}$ & $\mathrm{B2}^{\mathrm{b}}$ & $A 2^{\mathrm{b}}$ & $C 3^{\circ}$ & $\mathbf{B 3}^{\mathrm{c}}$ & $A 3^{c}$ & $\mathrm{CA}^{\mathrm{d}}$ & $\mathrm{BA}^{\mathrm{d}}$ & $A 4^{d}$ & $\mathrm{Cs}^{\circ}$ & $85^{\circ}$ & $A 5^{\circ}$ & $c 6^{\prime}$ & $B 6^{T}$ & $A 6^{\prime}$ \\
\hline Wet 19 & $8: 10$ & -0.076 & -24.0 & -28.6 & -27.4 & -48.3 & -47.2 & -46.0 & -53.8 & .54 .4 & .95 .8 & .65 .8 & .76 .9 & $-57,7$ & & 5.4 & -10.8 & 28.4 & .54 .1 & -68.18 \\
\hline $104 \%$ & $8: 20$ & -0.069 & -23.1 & -28.6 & -27.4 & -47.4 & -47.2 & -46.0 & .54 .7 & -55.2 & -94.9 & -65.9 & -76.0 & -57.7 & & 5.4 & -10.8 & 27.5 & .54 .1 & -68.48 \\
\hline $84 \%$ & $8: 30$ & -0.062 & -24.0 & -28.6 & -26.5 & -47.4 & .48 .1 & -46.9 & .53 .8 & -55.2 & .85 .8 & .65 .8 & -77.8 & -57.7 & & 5.4 & .10 .8 & 28.4 & .54 .1 & -66.48 \\
\hline$\% 1 / 4 \%$ & $8: 40$ & 0.056 & -24.0 & -28.6 & -27.4 & -47.4 & .47 .2 & -46.9 & .53 .8 & .54 .4 & .95 .8 & -65.9 & .78 .9 & -57.7 & & 5.4 & -10.0 & 27.5 & .54 .1 & -67.38 \\
\hline 24110 & 8:50 & 0.049 & -24.0 & -28.6 & -27.4 & -47.4 & -46.4 & -45.2 & .53 .8 & .54 .4 & -95.8 & -65.8 & -76.9 & -57.7 & & 5.4 & .10 .0 & 27.5 & .54 .1 & -68.18 \\
\hline$\% 4 \%$ & $9: 00$ & -0.042 & -24.0 & -27.7 & -27.4 & -47.4 & .47 .2 & -46.9 & .53 .8 & .54 .4 & -95.8 & -65.9 & -78.9 & -57.7 & & 4.5 & -10.0 & 27.5 & .54 .1 & -67.38 \\
\hline 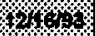 & $9: 10$ & 0.036 & -23.1 & -28.6 & -27.4 & -47.4 & -47.2 & -46.0 & .53 .8 & -55.2 & .95 .8 & -65.9 & -76.9 & -58.8 & & 5.4 & .10 .8 & 29.2 & .54 .1 & -68.18 \\
\hline$\%$ & $9: 20$ & -0.028 & -24.0 & -28.6 & -26.5 & -47.4 & .48 .1 & -46.0 & -52.9 & .54 .4 & -05.8 & -65.9 & -78.9 & -57.7 & & 5.4 & -10.9 & 27.4 & .54 .1 & -68.18 \\
\hline $124 \%$ & $9: 30$ & -0.021 & -23.1 & -28.6 & -27.4 & -47.4 & -47.2 & -46.0 & .53 .8 & .54 .4 & -95.8 & -65.8 & -76.9 & -56.9 & & 5.4 & .10 .8 & 28.3 & .54 .1 & -68.18 \\
\hline 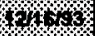 & $9: 40$ & -0.014 & -23.1 & -28.6 & -27.4 & -47.4 & .47 .2 & -46.9 & .54 .7 & -55.2 & -95.8 & .65 .1 & .78 .9 & -57.7 & & 6.2 & -11.7 & 27.4 & .54 .1 & -68.18 \\
\hline $34 \% \%$ & 9:50 & 0.007 & -23.1 & -27.7 & -27.4 & -47.4 & .47 .2 & -46.0 & .54 .7 & .55 .2 & -95.8 & .65 .8 & -78.9 & -56.8 & & 5.4 & .10 .8 & 28.3 & .55 .0 & $\begin{array}{l}-.67 .38 \\
\end{array}$ \\
\hline $101 \% \%$ & $10: 00$ & 0.000 & -24.0 & -28.6 & -25.7 & -47.4 & -47.2 & -46.8 & .52 .9 & .53 .5 & .95 .8 & -65.9 & -78.9 & -57.7 & & 6.2 & -10.8 & 28.3 & .55 .0 & -68.18 \\
\hline 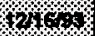 & $10: 10$ & 0.007 & -24.0 & -27.7 & -26.5 & -47.4 & -47.2 & -46.0 & .52 .8 & .55 .2 & .95 .8 & .65 .9 & -76.9 & -57.7 & & 5.4 & .10 .9 & 28.3 & .55 .0 & -69.08 \\
\hline 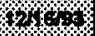 & $10: 20$ & 0.014 & -23.1 & -27.7 & -24.8 & -45.7 & .45 .5 & -46.0 & .52 .1 & .52 .6 & .85 .8 & -65.9 & .78 .9 & -57.8 & & 5.4 & .10 .0 & 28.3 & .55 .0 & -69.98 \\
\hline 5 & $10: 30$ & 0.021 & -23.1 & -28.9 & -28.5 & -47.4 & -46.4 & .45 .2 & .53 .8 & -55.2 & .05 .8 & -67.7 & -77.8 & -57.8 & & 4.5 & -10.8 & 27.4 & .55 .8 & -69.98 \\
\hline $14 \% 60$ & $10: 40$ & 0.028 & -21.4 & -28.0 & -24.8 & -45.7 & -44.7 & -45.2 & .52 .9 & .52 .6 & .94 .9 & -65.9 & -78.9 & -57.8 & & 0.2 & -10.9 & 26.5 & .55 .8 & .70 .78 \\
\hline 44169 & $10: 50$ & 0.035 & -21.4 & -26.9 & -24.8 & -44.8 & -44.7 & -46.0 & .52 .8 & .53 .5 & .94 .9 & .68 .0 & -75.2 & -56.8 & & 5.3 & -10.0 & 27.4 & .55 .8 & .71 .68 \\
\hline $14,49 x$ & $11: 00$ & 0.042 & -21.4 & .28 .0 & -23.9 & -45.7 & -44.7 & -45.2 & .52 .1 & .52 .6 & .94 .9 & -66.0 & .78 .9 & .57 .8 & & 0.2 & -10.9 & 28.5 & .55 .0 & .72 .58 \\
\hline 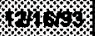 & $11: 10$ & 0.049 & -21.4 & -25.1 & -23.1 & -44.8 & -43.8 & -42.6 & .51 .2 & -51.7 & .94 .9 & .65 .1 & -74.3 & .57 .8 & & 6.2 & -10.0 & 27.4 & .56 .8 & .73 .38 \\
\hline $4 \% \%$ & $11: 20$ & 0.056 & -21.4 & -25.1 & -23.1 & -44.8 & -44.7 & -43.4 & .51 .2 & .51 .7 & .93 .2 & -64.2 & -73.5 & -56.0 & & 6.2 & -10.1 & 29.1 & .55 .9 & .73 .38 \\
\hline 3240. & 11:30 & 0.063 & -21.4 & -25.1 & -23.1 & -44.8 & -43.8 & -42.6 & .51 .2 & -51.7 & .94 .1 & -64.2 & -73.5 & -56.0 & & 0.2 & -10.1 & 27.4 & .55 .8 & -72.58 \\
\hline 174640 & $11: 40$ & 0.069 & -21.4 & -25.1 & .23 .1 & -44.8 & -43.8 & -42.6 & .51 .2 & .51 .7 & .94 .1 & -62.5 & -74.3 & -55.1 & & 6.2 & -10.1 & 29.1 & .55 .9 & -73.48 \\
\hline $35 \%$ & $11: 50$ & 0.076 & -21.4 & -24.3 & -23.1 & -44.8 & -43.8 & -42.6 & .52 .1 & .51 .7 & -94.1 & -64.2 & .74 .3 & -54.3 & & 7.0 & -0.2 & 27.4 & -56.8 & .73 .48 \\
\hline 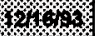 & $12: 00$ & 0.083 & -20.5 & -24.3 & -22.2 & -44.8 & -42.8 & -39.1 & .51 .2 & .50 .8 & -94.1 & $\begin{array}{l}-63.3 \\
\end{array}$ & -73.5 & -54.3 & -311.0 & 7.8 & -8.3 & 29.1 & .55 .8 & $\begin{array}{l}-73.48 \\
\end{array}$ \\
\hline 4769 & 12:10 & 0.090 & .20 .5 & -22.5 & .20 .4 & -44.8 & .43 .8 & .39 .1 & .50 .4 & -50.8 & -92.3 & -63.3 & .74 .3 & -53.4 & .220 .0 & 7.0 & .7 .5 & 27.3 & .55 .9 & -73.48 \\
\hline 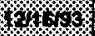 & 12:20 & 0.097 & -20.5 & -20.8 & .17 .8 & .43 .9 & -42.8 & -35.8 & .51 .2 & -48.2 & -91.5 & -62.5 & .72 .6 & -50.7 & -217.4 & 7.0 & .0 .8 & 28.2 & -55.9 & -74.28 \\
\hline $4 \%$ & $12: 30$ & 0.104 & -18.8 & -19.1 & .14 .3 & .43 .1 & .42 .1 & .32 .1 & .51 .2 & -48.2 & -80.6 & -61.6 & .71 .7 & -47.2 & .223 .4 & 7.9 & .5 .8 & 29.1 & -55.9 & -75.18 \\
\hline $184 \%$ & $12: 40$ & 0.111 & -19.6 & -13.9 & -9.1 & -43.9 & .39 .5 & .29 .5 & .51 .2 & -44.7 & -69.7 & -63.3 & .69 .1 & -47.2 & .239 .9 & 7.8 & -4.0 & 27.3 & -55.9 & .74 .28 \\
\hline 12406 & $12: 50$ & 0.118 & -17.9 & .9 .5 & .3 .9 & -43.8 & .36 .0 & -28.0 & .51 .2 & -41.1 & -88.0 & .68 .0 & -68.5 & .43 .7 & .220 .0 & 10.5 & -3.2 & 26.5 & .55 .8 & .75 .98 \\
\hline $1 \% 4 \%$ & $13: 00$ & 0.125 & -17.9 & .4 .3 & 1.4 & -42.2 & -30.0 & -21.6 & -49.5 & -34.9 & -86.2 & -64.2 & -60.4 & -41.1 & .231 .2 & 12.3 & -1.5 & 28.2 & -55.8 & .74 .28 \\
\hline $42714-\mathrm{x}$ & 13:10 & 0.132 & -17.9 & 9.7 & 8.3 & -41.3 & -24.0 & -17.3 & -47.8 & .30 .5 & -83.6 & .61 .6 & .55 .2 & -36.7 & .235 .6 & 14.0 & 0.3 & 28.2 & -55.8 & -75.18 \\
\hline 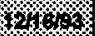 & $13: 20$ & 0.139 & -17.9 & 6.8 & 12.7 & -41.3 & -16.2 & -11.1 & -47.8 & .27 .0 & -82.8 & .61 .6 & .51 .7 & -33.2 & -240.8 & 14.9 & 1.1 & 29.1 & -55.0 & .75 .18 \\
\hline 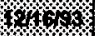 & $13: 30$ & 0.146 & -17.9 & 11.3 & 15.3 & -40.5 & -13.6 & -8.4 & -47.8 & -23.5 & -81.9 & -61.8 & -49.1 & .29 .7 & .243 .4 & 18.6 & 3.7 & 20.1 & -54.2 & -73.48 \\
\hline $14 x_{1}$ & $13: 40$ & 0.153 & -17.0 & 13.9 & 18.8 & -38.7 & -11.9 & .7 .7 & -47.8 & -19.9 & -81.0 & .62 .5 & -45.7 & -27.9 & .244 .2 & 17.5 & 4.6 & 28.2 & -54.2 & .72 .58 \\
\hline $174 \%$ & $13: 50$ & 0.100 & -14.4 & 15.6 & 20.5 & -37.9 & -8.5 & -4.2 & -46.0 & -18.1 & -81.0 & -60.7 & .43 .1 & -26.2 & .246 .8 & 18.3 & 6.3 & 29.9 & -52.4 & -73.48 \\
\hline 12410.4 & $14: 00$ & 0.167 & -14.4 & 19.1 & 22.3 & .34 .4 & -5.0 & .0 .7 & -46.0 & -16.4 & -81.0 & .59 .8 & .41 .3 & -25.3 & .249 .4 & 19.2 & 7.2 & 29.8 & -52.4 & .72 .58 \\
\hline $42 \% 1 \%$ & \begin{tabular}{|l|}
$14: 10$ \\
\end{tabular} & 0.174 & -11.0 & 20.8 & 24.0 & .30 .8 & .3 .3 & 1.1 & .43 .4 & .13 .7 & .79 .3 & -58.1 & -38.7 & .23 .5 & .252 .0 & 21.0 & 8.0 & 29.9 & -51.6 & .71 .78 \\
\hline 52 & $14: 20$ & 0.181 & -4.0 & 22.5 & 25.8 & .24 .0 & 0.2 & 2.8 & -40.0 & -11.8 & -79.3 & -57.2 & .37 .8 & -22.7 & .252 .0 & 21.0 & 9.8 & 28.1 & -50.7 & .71 .78 \\
\hline
\end{tabular}


Table 5. Tensiometer Data

\begin{tabular}{|c|c|c|c|c|c|c|c|c|c|c|c|c|c|c|c|c|c|c|c|c|}
\hline & & & & & & & & & Tens & siometer & Data & & & & & & & & & \\
\hline & & Test & $\begin{array}{c}\text { Soll } \\
\text { Moisture } \\
\text { Tension } \\
\left(\mathrm{Cm} \mathrm{H}_{2} \mathrm{O}\right) \\
\end{array}$ & $\begin{array}{c}\text { Soll } \\
\text { Molsture } \\
\text { Tension } \\
\left(\mathrm{cm} \mathrm{H}_{2} \mathrm{O}\right) \\
\end{array}$ & $\begin{array}{c}\text { Soil } \\
\text { Molsture } \\
\text { Tension } \\
\left(\mathrm{cm} \mathrm{H}_{2} \mathrm{O}\right) \\
\end{array}$ & $\begin{array}{c}\text { Soll } \\
\text { Molsture } \\
\text { Tonsion } \\
\left(\mathrm{Cm} \mathrm{H}_{2} \mathrm{O}\right) \\
\end{array}$ & $\begin{array}{c}\text { Soll } \\
\text { Moisture } \\
\text { Tenston } \\
\left(\mathrm{cm} \mathrm{H}_{2} \mathrm{O}\right) \\
\end{array}$ & $\begin{array}{c}\text { Soil } \\
\text { Molsture } \\
\text { Tonslon } \\
\left.\text { (cm } \mathrm{H}_{2} \mathrm{O}\right) \\
\end{array}$ & $\begin{array}{c}\text { Soll } \\
\text { Molsture } \\
\text { Tension } \\
\left(\mathrm{Cm} \mathrm{H}_{2} \mathrm{O}\right)\end{array}$ & $\begin{array}{c}\text { Soll } \\
\text { Molsture } \\
\text { Tension } \\
\text { (em Hz) } \\
\end{array}$ & $\begin{array}{c}\text { Soll } \\
\text { Moisture } \\
\text { Tension } \\
\left(\mathrm{cm} \mathrm{H} \mathrm{H}_{2} \mathrm{O}\right) \\
\end{array}$ & $\begin{array}{c}\text { Soll } \\
\text { Moisture } \\
\text { Tension } \\
\left(\mathrm{cm} \mathrm{H}_{2} \mathrm{O}\right) \\
\end{array}$ & $\begin{array}{c}\text { Soll } \\
\text { Moisture } \\
\text { Tension } \\
\left(\mathrm{Cm} \mathrm{H}_{2} \mathrm{O}\right) \\
\end{array}$ & $\begin{array}{c}\text { Soll } \\
\text { Moisture } \\
\text { Tension } \\
\left(\mathrm{Cm} \mathrm{H}_{2} \mathrm{O}\right) \\
\end{array}$ & $\begin{array}{c}\text { Soll } \\
\text { Moisture } \\
\text { Tansion } \\
\left(\mathrm{cm} \mathrm{H}_{2} \mathrm{O}\right) \\
\end{array}$ & $\begin{array}{c}\text { Soll } \\
\text { Moisture } \\
\text { Tenslon } \\
(\mathrm{Cm} \mathrm{H} \mathrm{O})\end{array}$ & $\begin{array}{c}\text { Soll } \\
\text { Moisture } \\
\text { Tension } \\
\left(\mathrm{Cm} \mathrm{H}_{2} \mathrm{O}\right) \\
\end{array}$ & $\begin{array}{c}\text { Soll } \\
\text { Molsture } \\
\text { Tension } \\
\left(\mathrm{cm}_{2} \mathrm{O} \mathrm{O}\right) \\
\end{array}$ & $\begin{array}{c}\text { Soll } \\
\text { Moleture } \\
\text { Tansion } \\
\left(\mathrm{Cm} \mathrm{H}_{2} \mathrm{O}\right) \\
\end{array}$ & $\begin{array}{c}\text { Soll } \\
\text { Mobsture } \\
\text { Tenston } \\
(\mathrm{cm} \mathrm{H}, \mathrm{O}) \\
\end{array}$ \\
\hline Date & Time & Days ${ }^{1}$ & $C 1^{\prime \prime}$ & B1" & $A 1^{2}$ & $\mathrm{Cl}^{\mathrm{b}}$ & $B 2^{\mathrm{b}}$ & $A 2^{6}$ & $\mathrm{C3}^{\mathrm{c}}$ & $\mathrm{B3}^{\mathrm{c}}$ & $A 3^{c}$ & $C 4^{d}$ & $B 4^{d}$ & $\overline{A 4^{d}}$ & $C 5^{\circ}$ & $B 5^{\circ}$ & $\mathrm{A}^{\circ}$ & $\mathrm{Co}^{\prime}$ & $\mathrm{B}^{\prime}$ & $A 6^{\prime}$ \\
\hline 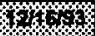 & 14:30 & 0.188 & 6.4 & 28.0 & 27.5 & -17.0 & 3.6 & 6.3 & .33 .0 & -11.1 & -79.3 & .54 .6 & -37.0 & -20.8 & -248.6 & 22.7 & 9.8 & 30.8 & -49.8 & .71 .78 \\
\hline 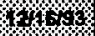 & $14: 40$ & 0.194 & 23.8 & 26.9 & 29.3 & -0.5 & 5.3 & 6.3 & .20 .0 & -10.2 & .79 .3 & -47.6 & -32.7 & -19.2 & -245.1 & 23.6 & 10.8 & 31.7 & -49.0 & -09.98 \\
\hline 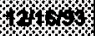 & $14: 50$ & 0.201 & 34.2 & 27.7 & 31.0 & 7.3 & 7.1 & 9.8 & .7 .0 & .7 .5 & .78 .4 & -36.2 & -31.8 & -18.3 & -238.2 & 24.4 & 11.5 & 33.4 & -49.0 & -09.98 \\
\hline $1 \%, \%$ & 15:00 & 0.208 & 33.3 & 29.5 & 30.1 & 7.3 & 7.8 & 9.8 & -6.2 & .5 .8 & .78 .4 & -28.4 & -30.1 & -17.4 & -231.2 & 24.4 & 12.3 & 35.2 & -47.2 & -09.98 \\
\hline 171611 & $15: 10$ & 0.216 & 34.2 & 29.5 & 32.7 & 7.3 & 8.8 & 9.8 & .4 .4 & -4.9 & .78 .4 & -24.8 & -30.1 & -17.4 & -228.6 & 24.4 & 13.2 & 36.8 & -47.2 & -69.88 \\
\hline 134,4 & 15:20 & 0.222 & 34.2 & 30.3 & 32.7 & 8.0 & 10.5 & 9.8 & -1.8 & -4.9 & -79.3 & .23 .1 & -29.2 & -15.7 & -223.4 & 25.3 & 13.2 & 37.8 & .47 .2 & -69.18 \\
\hline 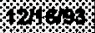 & $15: 30$ & 0.229 & 35.0 & 31.2 & 32.7 & 10.7 & 11.3 & 9.8 & -1.0 & -5.8 & .78 .4 & -22.3 & -28.3 & -15.7 & -223.4 & 27.1 & 14.1 & 40.4 & $.44,6$ & 68.28 \\
\hline $12 \% 16$ & \begin{tabular}{|l|}
$15: 40$ \\
\end{tabular} & 0.236 & 37.7 & 32.9 & 32.7 & 11.6 & 12.2 & 12.4 & 0.8 & -3.1 & .78 .4 & -20.5 & -27.5 & -15.7 & -218.2 & 27.1 & 14.1 & 40.4 & .45 .5 & -68.28 \\
\hline 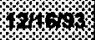 & $15: 50$ & 0.243 & 37.7 & 32.8 & 35.4 & 14.2 & 13.0 & 13.2 & 1.8 & -2.2 & .78 .4 & -20.5 & -28.6 & -14.8 & -217.3 & 26.2 & 14.1 & 41.3 & -42.9 & -86.28 \\
\hline 0.1469 & 16:00 & 0.250 & 41.1 & 34.7 & 36.2 & 15.1 & 13.0 & 13.2 & 3.3 & -1.3 & -78.4 & -18.8 & -25.7 & -13.8 & -213.0 & 27.9 & 14.8 & 42.2 & .43 .7 & -88.28 \\
\hline 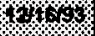 & $16: 10$ & 0.257 & 41.1 & 34.7 & 36.2 & 15.8 & 15.6 & 15.0 & 4.2 & -1.3 & .78 .4 & -17.9 & -25.7 & -13.0 & -209.5 & 27.9 & 14.9 & 43.8 & .43 .8 & .68 .28 \\
\hline $124=61$ & $18: 20$ & 0.264 & 41.1 & 36.4 & 38.8 & 17.7 & 16.5 & 15.9 & 5.1 & 1.3 & .78 .4 & -17.0 & -24.0 & -13.0 & .207 .8 & 27.9 & 14.9 & 44.8 & .43 .8 & 68.28 \\
\hline 172,601 & $16: 30$ & 0.271 & 44.8 & 36.4 & 39.7 & 18.5 & 17.3 & 17.8 & 6.8 & 2.2 & .78 .4 & -15.3 & -23.1 & -12.2 & -202.8 & 27.8 & 14.9 & 44.8 & -43.8 & 68.28 \\
\hline 19409 & $16: 40$ & 0.278 & 44.6 & 37.3 & 39.7 & 18.4 & 18.2 & 18.5 & 7.7 & 2.2 & -78.4 & -12.7 & .23 .1 & -12.2 & -200.0 & 27.9 & 16.6 & 46.5 & .42 .0 & -68.28 \\
\hline 824001 & $16: 50$ & 0.285 & 44.8 & 38.1 & 39.7 & 22.0 & 19.1 & 17.6 & 8.5 & 4.8 & -77.6 & 8.3 & -22.3 & -11.3 & -296.3 & 29.6 & 16.6 & 49.1 & -40.3 & -66.58 \\
\hline 814646 & 17:00 & 0.292 & 45.4 & 39.8 & 39.7 & 22.0 & 19.8 & 19.3 & 8.5 & 4.8 & .78 .4 & -8.3 & -20.5 & -10.4 & -454.1 & 27.9 & 18.4 & 48.3 & .40 .3 & .65 .68 \\
\hline $17 \times$ & $\begin{array}{l}17: 10 \\
\end{array}$ & 0.299 & 45.4 & 39.8 & 41.4 & 22.0 & 19.1 & 18.5 & 10.3 & 4.8 & .78 .4 & 8.3 & .21 .4 & -9.5 & -439.4 & 29.7 & 16.6 & 49.1 & -40.3 & 65.68 \\
\hline $14+5.8$ & $17: 20$ & 0.300 & 48.0 & 40.7 & 41.4 & 23.7 & 22.5 & 18.4 & 12.0 & 5.7 & -79.3 & -7.4 & -20.5 & -8.7 & -442.8 & 28.8 & 16.6 & 50.0 & -40.3 & 86.58 \\
\hline $1 \% 1 \%$ & $17: 30$ & 0.313 & 48.0 & 42.4 & 42.3 & 23.7 & 21.7 & 19.4 & 11.1 & 5.7 & -80.2 & -7.4 & -19.7 & -8.7 & -447.2 & 28.8 & 17.5 & 48.3 & -43.8 & -68.28 \\
\hline 5416. & $17: 40$ & 0.319 & 49.8 & 42.4 & 43.2 & 24.6 & 23.4 & 23.7 & 10.3 & 6.8 & -80.2 & -8.3 & -18.8 & 8.6 & -442.0 & 29.7 & 18.4 & 49.2 & -40.3 & -66.58 \\
\hline 12169 & $17: 50$ & 0.326 & 50.7 & 42.5 & 44.1 & 28.4 & 23.4 & 23.7 & 12.0 & 7.5 & -80.2 & .7 .4 & -17.9 & .7 .8 & -438.5 & 31.4 & 17.5 & 48.3 & -40.3 & -67.38 \\
\hline 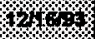 & \begin{tabular}{|l|}
$18: 00$ \\
\end{tabular} & 0.333 & 52.4 & 44.2 & 44.9 & 29.0 & 25.1 & 24.6 & 13.7 & 8.4 & -80.2 & -6.5 & -17.9 & -6.0 & 15.2 & 31.4 & 18.4 & 49.2 & -39.4 & -67.38 \\
\hline $1 \% 164$ & 18:10 & 0.340 & 52.4 & 44.2 & 46.7 & 29.8 & 26.8 & 24.6 & 14.6 & 8.4 & 80.2 & -5.7 & -16.2 & -6.0 & 16.8 & 31.4 & 20.1 & 50.8 & -39.4 & -66.58 \\
\hline 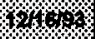 & $18: 20$ & 0.347 & 54.1 & 45.9 & 46.7 & 29.8 & 28.6 & 26.3 & 15.5 & 10.1 & -80.2 & -4.8 & -15.3 & -4.3 & 16.1 & 33.2 & 20.1 & 51.0 & -37.6 & -60.58 \\
\hline $12 \%$ & $18: 30$ & 0.364 & 54.1 & 46.8 & 47.6 & 31.6 & 28.6 & 26.3 & 17.2 & 11.0 & -80.2 & -3.9 & -15.3 & -4.3 & 16.1 & 33.2 & 19.3 & 52.7 & -38.5 & -67.38 \\
\hline 17401 & $18: 40$ & 0.361 & 54.1 & 47.7 & 49.3 & 32.4 & 28.6 & 28.1 & 18.1 & 11.9 & -80.2 & -3.9 & -14.4 & -3.4 & 17.8 & 32.3 & 20.1 & 51.8 & -39.4 & -66.58 \\
\hline \%3:4 & $18: 50$ & 0.368 & 54.1 & 47.7 & 49.3 & 32.4 & 31.2 & 29.0 & 19.0 & 13.7 & -80.2 & -3.0 & -14.4 & .3 .4 & 17.8 & 34.1 & 21.0 & 53.6 & -38.5 & -68.58 \\
\hline 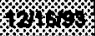 & $19: 00$ & 0.375 & 55.8 & 49.4 & 50.2 & 33.3 & 32.0 & 29.8 & 19.8 & 13.7 & -79.3 & -1.3 & -13.8 & .1 .8 & 17.8 & 33.2 & 21.8 & 53.8 & -37.8 & -66.58 \\
\hline 174,4 & $\begin{array}{l}9: 10 \\
\end{array}$ & 0.382 & 59.4 & 50.3 & 50.2 & 34.2 & 32.0 & 30.7 & 20.7 & 13.7 & .79 .3 & -0.4 & .12 .7 & -1.8 & 18.7 & 33.2 & 22.7 & 53.6 & -37.6 & -86.58 \\
\hline 17464 & $19: 20$ & 0.389 & 59.4 & 51.1 & 51.1 & 35.9 & 32.0 & 30.7 & 21.6 & 13.7 & -80.2 & 0.5 & .11 .0 & 0.1 & 18.7 & 34.1 & 22.7 & 54.5 & -37.6 & -66.58 \\
\hline $1210 \%$ & 19:30 & 0.396 & 59.4 & 50.3 & 51.9 & 35.9 & 32.0 & 32.5 & 21.6 & 16.3 & -80.2 & 1.3 & .11 .0 & -0.7 & 21.3 & 34.1 & 23.6 & 55.4 & -37.6 & -68.58 \\
\hline 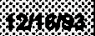 & $19: 40$ & 0.403 & 61.1 & 51.1 & 52.8 & 35.8 & 34.6 & 32.5 & 22.4 & 17.2 & -80.2 & 2.2 & .10 .8 & -0.7 & 21.3 & 34.1 & 23.6 & 55.4 & -36.7 & 66.58 \\
\hline 6 $7 \%$ & $19: 50$ & 0.410 & 60.2 & 51.1 & 52.8 & 36.8 & 34.6 & 33.3 & 23.3 & 17.2 & .79 .3 & 3.1 & -10.4 & -0.7 & 20.4 & 34.1 & 23.6 & 55.4 & -35.9 & -68.28 \\
\hline \% & $20: 00$ & 0.417 & 61.1 & 52.8 & 51.9 & 36.8 & 34.6 & 34.2 & 24.2 & 17.2 & -80.2 & 4.0 & -9.2 & 0.1 & 20.4 & 33.2 & 23.6 & 55.4 & 36.7 & -66.48 \\
\hline $5 \%$ & $20: 10$ & 0.424 & 62.8 & 52.8 & 54.5 & 38.5 & 35.5 & 34.2 & 24.2 & 18.1 & -81.0 & 4.0 & -10.1 & 1.0 & 21.3 & 35.0 & 22.8 & 56.3 & -35.9 & 60.48 \\
\hline $121 \%$ & 20:20 & 0.431 & 62.8 & 52.8 & 56.3 & 38.6 & 36.4 & 36.0 & 25.9 & 18.1 & 81.0 & 4.0 & -8.3 & 1.8 & 22.2 & 35.8 & 23.6 & 57.1 & -35.9 & -87.38 \\
\hline $17 \% 1 \%$ & $20: 30$ & 0.438 & 63.7 & 55.5 & 58.3 & 39.4 & 36.4 & 36.0 & 24.2 & 18.8 & 81.0 & 4.8 & -7.5 & 2.8 & 23.0 & 35.8 & 24.5 & 58.0 & -35.8 & 88.48 \\
\hline 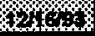 & $20: 40$ & 0.444 & 83.7 & 58.4 & 56.3 & 39.4 & 38.1 & 36.0 & 25.8 & 20.8 & 81.0 & 6.6 & -7.5 & 2.8 & 23.1 & 35.9 & 25.4 & 58.0 & -35.0 & -66.48 \\
\hline
\end{tabular}


Table 5. Tensiometer Data

\begin{tabular}{|c|c|c|c|c|c|c|c|c|c|c|c|c|c|c|c|c|c|c|c|c|}
\hline & & & & & & & & & Ten & siometer & Data & & & & & & & & & \\
\hline & & Test & $\begin{array}{c}\text { Soll } \\
\text { Molsture } \\
\text { Tension } \\
\left(\mathrm{cm} \mathrm{H}_{2} \mathrm{O}\right) \\
\end{array}$ & $\begin{array}{c}\text { Soll } \\
\text { Molsture } \\
\text { Tension } \\
\text { (cm } \mathrm{H}_{2} \mathrm{O} \text { ) } \\
\end{array}$ & \begin{tabular}{|c|} 
Soll \\
Molsture \\
Tension \\
(em $\left.\mathrm{H}_{2} \mathrm{O}\right)$ \\
\end{tabular} & 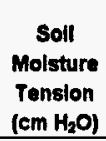 & $\begin{array}{c}\text { Soll } \\
\text { Molsture } \\
\text { Tension } \\
\left(\mathrm{cm} \mathrm{H}_{2} \mathrm{O}\right) \\
\end{array}$ & $\begin{array}{c}\text { Soll } \\
\text { Molsture } \\
\text { Tonsion } \\
\left(\mathrm{Cm} \mathrm{H}_{2} \mathrm{O}\right) \\
\end{array}$ & \begin{tabular}{|c|} 
Soll \\
Moisture \\
Tension \\
(em $\mathrm{H}_{2} \mathrm{O}$ ) \\
\end{tabular} & 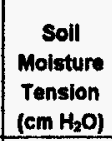 & $\begin{array}{c}\text { Soil } \\
\text { Moisture } \\
\text { Tension } \\
\left(\mathrm{Cm} \mathrm{H}_{2} \mathrm{O}\right) \\
\end{array}$ & $\begin{array}{c}\text { Soll } \\
\text { Molsture } \\
\text { Tension } \\
\left(\mathrm{Cm} \mathrm{H}_{2} \mathrm{O}\right) \\
\end{array}$ & $\begin{array}{c}\text { Soll } \\
\text { Molsture } \\
\text { Tenston } \\
\left(\mathrm{Cm} \mathrm{H}_{2} \mathrm{O}\right) \\
\end{array}$ & $\begin{array}{c}\text { Soll } \\
\text { Molsturn } \\
\text { Tension } \\
\left(\mathrm{Cm} \mathrm{H}_{2} \mathrm{O}\right) \\
\end{array}$ & $\begin{array}{c}\text { Soll } \\
\text { Molsture } \\
\text { Tension } \\
\left(\mathrm{cm} \mathrm{H} \mathrm{H}_{2} \mathrm{O}\right)\end{array}$ & $\begin{array}{c}\text { Soll } \\
\text { Molsture } \\
\text { Tenston } \\
\left(\mathrm{Cm} \mathrm{H}_{2} \mathrm{O}\right) \\
\end{array}$ & $\begin{array}{c}\text { Soll } \\
\text { Moisture } \\
\text { Tanslon } \\
\left(\mathrm{cm} \mathrm{H}_{2} \mathrm{O}\right) \\
\end{array}$ & $\begin{array}{c}\text { Soll } \\
\text { Molsture } \\
\text { Tension } \\
\left(\mathrm{cm} \mathrm{H}_{2} \mathrm{O}\right)\end{array}$ & $\begin{array}{c}\text { Soll } \\
\text { Molsture } \\
\text { Tension } \\
\left(\mathrm{cm} \mathrm{H}_{2} \mathrm{O}\right) \\
\end{array}$ & $\begin{array}{c}\text { Soll } \\
\text { Molsture } \\
\text { Tension } \\
\left(\mathrm{Cm} \mathrm{H}_{2} \mathrm{O}\right) \\
\end{array}$ \\
\hline Date & Time & Days' & $C 1^{n}$ & $B 1^{\prime \prime}$ & $A 1^{\prime \prime}$ & $\mathrm{C2}^{\mathrm{b}}$ & $B 2^{b}$ & $A 2^{b}$ & $\mathrm{C3}^{\mathrm{C}}$ & $\mathrm{B3}^{\circ}$ & $A 3^{c}$ & $C 4^{d}$ & $B 4^{d}$ & $A 4^{d}$ & $C \sigma^{\circ}$ & $B 5^{\circ}$ & $A 5^{\circ}$ & $\mathrm{Co}^{\prime}$ & $B 6^{\prime}$ & $A 6^{\prime}$ \\
\hline 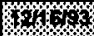 & $20: 50$ & 0.451 & 63.7 & 55.5 & 57.2 & 38.6 & 37.2 & 36.8 & 28.8 & 19.9 & -81.8 & 5.7 & -7.5 & 3.7 & 23.9 & 36.7 & 26.2 & 58.0 & -35.8 & -67.38 \\
\hline 1.6 & $21: 00$ & 0.456 & 65.5 & 58.1 & 58.0 & 39.4 & 38.9 & 36.8 & 27.7 & 20.8 & -81.0 & 8.6 & .6 .6 & 2.8 & 23.9 & 38.7 & 26.2 & 58.0 & -35.8 & -68.48 \\
\hline $801 \%$ & 21:10 & 0.465 & 64.6 & 57.2 & 58.9 & 40.3 & 38.1 & 37.7 & 27.7 & 20.8 & -81.8 & 7.5 & 6.6 & 3.7 & 23.9 & 36.7 & 27.1 & 58.0 & -34.1 & -07.38 \\
\hline 12169 & $21: 20$ & 0.472 & 65.5 & 58.1 & 58.9 & 41.2 & 39.0 & 38.6 & 27.7 & 21.6 & & 7.5 & -5.7 & 3.7 & 23.9 & 38.7 & 26.2 & 58.0 & -35.8 & -88.18 \\
\hline $4246 \%$ & 21:30 & 0.479 & 66.3 & 58.1 & 59.8 & 42.0 & 39.0 & 41.2 & 27.7 & 21.7 & & 7.5 & -4.0 & 5.4 & 24.8 & 37.6 & 28.3 & 58.1 & .35 .0 & -67.38 \\
\hline $15 \%$ & 21:40 & 0.486 & 67.2 & 58.1 & 59.8 & 42.0 & 39.8 & 39.5 & 27.7 & 21.7 & & 8.2 & 4.8 & 5.4 & 24.8 & 37.6 & 27.1 & 58.9 & -35.0 & -87.38 \\
\hline 1\%10 & 21:50 & 0.493 & 86.3 & 59.0 & 59.8 & 42.9 & 39.0 & 38.6 & 28.5 & 24.3 & & 8.4 & 4.0 & 6.3 & 24.8 & 37.6 & 27.1 & 58.8 & -35.0 & -68.18 \\
\hline $1 \% 1 \%$ & $22: 00$ & 0.500 & 67.2 & 59.8 & 60.7 & 42.9 & 41.5 & 40.3 & 29.4 & 24.3 & & 10.1 & -4.0 & 6.3 & 25.7 & 37.6 & 28.8 & 60.7 & -35.0 & -68.18 \\
\hline 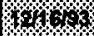 & $22: 10$ & 0.507 & 68.1 & 59.0 & 60.7 & 42.9 & 41.5 & 40.3 & 30.3 & 23.4 & & 11.0 & 3.1 & 8.3 & 28.5 & 38.5 & 28.0 & 81.6 & .33 .2 & -68.18 \\
\hline $10 \%$ & 22:20 & 0.514 & 68.9 & 60.7 & 62.4 & 43.8 & 42.4 & 41.2 & 30.3 & 25.2 & & 11.0 & .2 .2 & 6.3 & 25.7 & 38.5 & 28.0 & 61.6 & -34.8 & -68.18 \\
\hline $142,6 \%$ & 22:30 & 0.521 & 68.9 & 69.6 & 62.4 & 42.9 & 42.4 & 41.2 & 31.1 & 25.2 & & 11.0 & .2 .2 & 6.3 & 28.5 & 38.5 & 28.8 & 81.6 & -34.1 & -68.18 \\
\hline 1246 & 22:40 & 0.528 & 68.9 & 62.4 & 63.3 & 45.5 & 42.4 & 41.2 & 31.1 & 25.2 & & 11.9 & .2 .2 & 7.2 & 27.4 & 37.6 & 30.8 & 62.5 & -34.1 & -68.18 \\
\hline 12,00 & $22: 50$ & 0.536 & 68.9 & 64.6 & 63.3 & 46.4 & 42.4 & 42.1 & 31.1 & 28.1 & & 11.0 & 0.4 & 8.9 & 28.3 & 39.4 & 30.8 & 61.6 & -33.2 & -68.18 \\
\hline$\%$ & $23: 00$ & 0.542 & 69.8 & 62.4 & 63.3 & 46.4 & 43.3 & 43.0 & 32.0 & 27.8 & & 12.7 & -0.5 & 8.8 & 27.4 & 38.5 & 30.8 & 60.7 & -33.2 & -68.18 \\
\hline $421 \%$ & 23:10 & 0.549 & 69.8 & 61.6 & 84.2 & 45.5 & 44.1 & 43.0 & 32.0 & 27.8 & & 12.7 & .0 .5 & 8.1 & 27.4 & 39.4 & 29.7 & 61.6 & -34.1 & -68.18 \\
\hline \%2. & $23: 20$ & 0.566 & 69.8 & 63.3 & 63.3 & 46.4 & 43.3 & 43.8 & 33.7 & 26.1 & & 14.5 & -0.5 & 9.8 & 28.3 & 38.5 & 30.8 & 61.6 & -32.3 & -67.38 \\
\hline $1 \% 69$ & $23: 30$ & 0.563 & 69.8 & 64.2 & 63.3 & 46.4 & 45.0 & 44.7 & 33.7 & 28.7 & & 13.6 & -1.4 & 8.8 & 29.1 & 40.3 & 30.8 & 61.6 & -33.2 & -68.18 \\
\hline $10 \%$ & $23: 40$ & 0.569 & 69.0 & 63.3 & 64.2 & 47.3 & 45.0 & 44.7 & 32.8 & 28.7 & & 14.5 & 1.3 & 9.8 & 29.2 & 40.3 & 30.8 & 63.3 & -33.2 & -68.18 \\
\hline $1216 \%$ & $23: 50$ & 0.576 & 71.6 & 64.2 & 65.1 & 47.3 & 45.8 & 45.6 & 33.7 & 28.7 & & 14.5 & 1.3 & 10.7 & 29.2 & 40.3 & 30.6 & 63.3 & -33.2 & -69.08 \\
\hline $1247 \%$ & $0: 00$ & 0.583 & 70.7 & 65.1 & 65.9 & 47.3 & 45.0 & 44.7 & 33.7 & 29.6 & & 14.5 & 1.3 & 8.8 & 28.2 & 41.1 & 30.6 & 63.3 & -33.2 & -68.18 \\
\hline 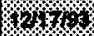 & $0: 10$ & 0.590 & 72.4 & 65.1 & 65.1 & 49.0 & 45.8 & 45.6 & 34.6 & 28.7 & & 15.4 & 2.1 & 10.7 & 29.2 & 40.3 & 30.6 & 64.2 & -33.2 & -09.08 \\
\hline $1 \%$ & $0: 20$ & 0.597 & 72.4 & 65.1 & 65.8 & 49.0 & 45.8 & 46.4 & 34.6 & 30.5 & & 15.4 & 2.1 & 11.6 & 29.2 & 41.1 & 31.5 & 65.1 & -33.2 & -69.88 \\
\hline 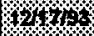 & $0: 30$ & 0.004 & 72,4 & 65.1 & 66.8 & 49.0 & 46.7 & 46.5 & 35.5 & 31.4 & & 18.2 & 2.1 & 11.6 & 30.0 & 41.1 & 31.5 & 64.2 & -33.2 & -09.08 \\
\hline 12471 & $0: 40$ & 0.611 & 72.4 & 65.9 & 65.8 & 49.0 & 47.6 & 47.3 & 36.4 & 31.4 & & 17.1 & 1.3 & 12.5 & 30.8 & 41.1 & 31.5 & 64.2 & -33.2 & -69.08 \\
\hline 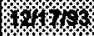 & $0: 50$ & 0.618 & 72.4 & 66.8 & 86.8 & 49.0 & 48.5 & 48.2 & 35.5 & 31.4 & & 18.0 & 2.1 & 12.5 & 30.9 & 42.0 & 32.3 & 65.1 & -32.3 & -68.18 \\
\hline 17,199 & $1: 00$ & 0.626 & 73.3 & 65.9 & 66.8 & 49.9 & 48.5 & 48.2 & 35.5 & 32.3 & & 18.0 & 3.0 & 12.5 & 31.8 & 42.0 & 31.5 & 68.0 & -33.2 & -69.08 \\
\hline 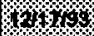 & 1:10 & 0.032 & 73.3 & 67.7 & 67.7 & 49.9 & 47.6 & 48.2 & 36.4 & 32.3 & & 18.0 & 3.9 & 13.3 & 31.8 & 42.0 & 33.2 & 68.0 & -33.2 & -69.08 \\
\hline $14 \% 10$ & $1: 20$ & 0.639 & 73.3 & 65.9 & 67.7 & 50.8 & 49.3 & 48.2 & 36.4 & 32.3 & & 18.0 & 3.8 & 13.3 & 30.9 & 42.0 & 33.2 & 68.0 & .33 .2 & -69.08 \\
\hline 12414 & 1:30 & 0.046 & 74.2 & 67.7 & 67.7 & 50.8 & 49.3 & 49.1 & 37.2 & 33.2 & & 18.9 & 4.7 & 13.3 & 32.6 & 42.0 & 34.1 & 68.0 & 32.3 & -69.08 \\
\hline 1541,1 & $1: 40$ & 0.653 & 74.2 & 67.7 & 68.5 & 51.6 & 50.2 & 49.1 & 38.1 & 32.3 & & 18.0 & 4.7 & 13.3 & 32.6 & 42.9 & 34.1 & 68.0 & 32.3 & -69.88 \\
\hline $534 \% 1$ & $1: 50$ & 0.660 & 74.2 & 68.5 & 66.8 & 51.6 & 50.2 & 49.8 & 39.0 & 34.0 & & 19.7 & 4.7 & 15.1 & 32.6 & 42.0 & 33.2 & 68.9 & -32.3 & -69.88 \\
\hline \% & 2:00 & 0.667 & 75.0 & 67.7 & 68.5 & 52.5 & 50.2 & 49.9 & 39.0 & 34.0 & & 18.9 & 5.6 & 16.0 & 32.6 & 42.8 & 34.1 & 68.9 & -31.4 & -69.88 \\
\hline (1. & $2: 10$ & 0.674 & 75.8 & 66.8 & 68.6 & 52.5 & 50.2 & 50.8 & 37.2 & 34.9 & & 19.7 & 5.6 & 16.0 & 33.5 & 42.0 & 34.0 & 68.9 & -30.6 & -69.88 \\
\hline $1 \% 1 \%$ & $2: 20$ & 0.081 & 75.8 & 68.5 & 68.6 & 52.5 & 51.1 & 51.7 & 39.0 & 34.9 & & 19.7 & 6.5 & 16.8 & 33.5 & 43.8 & 34.8 & 68.9 & -31.4 & -9.88 \\
\hline \%o & $2: 30$ & 0.688 & 75.8 & 70.3 & 69.4 & 52.5 & 51.1 & 51.7 & 39.0 & 34.9 & & 21.5 & 6.5 & 16.0 & 34.4 & $\Delta 3.8$ & 34.8 & 68.6 & -32.3 & .70 .78 \\
\hline 12470 & $2: 40$ & 0.094 & 75.9 & 68.5 & 70.3 & 52.5 & 51.1 & 51.7 & 38.1 & 35.8 & & 21.5 & 7.3 & 16.8 & 33.5 & .4 .7 & 34.8 & 68.6 & 31.4 & 69.88 \\
\hline 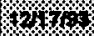 & $2: 50$ & 0.701 & 75.9 & 69.4 & 70.3 & 53.4 & 51.1 & 52.6 & 39.0 & 37.6 & & 22.4 & 7.3 & 16.8 & 35.2 & 43.8 & 34.8 & 00.5 & -31.4 & -69.88 \\
\hline 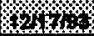 & $3: 00$ & 0.708 & 75.9 & 71.1 & 71.2 & 53.4 & 51.8 & 51.7 & 40.7 & 35.8 & & 21.5 & 8.2 & 16.8 & 35.2 & 44.7 & 34.8 & 68.6 & -31.4 & .71 .68 \\
\hline
\end{tabular}


Table 5. Tensiometer Data

\begin{tabular}{|c|c|c|c|c|c|c|c|c|c|c|c|c|c|c|c|c|c|c|c|c|}
\hline & & & & & & & & & Ten & siometer & Data & & & & & & & & & \\
\hline & & Teast & $\begin{array}{c}\text { Soil } \\
\text { Moisture } \\
\text { Tension } \\
\left(\mathrm{Cm} \mathrm{H}_{2} \mathrm{O}\right)\end{array}$ & $\begin{array}{c}\text { Soil } \\
\text { Moisture } \\
\text { Tension } \\
\left(\mathrm{C} \mathrm{H}_{2} \mathrm{O}\right) \\
\end{array}$ & $\begin{array}{c}\text { Soll } \\
\text { Moisture } \\
\text { Tension } \\
\left(\mathrm{Cm} \mathrm{H}_{2} \mathrm{O}\right)\end{array}$ & $\begin{array}{c}\text { Soil } \\
\text { Molsture } \\
\text { Tension } \\
\left(\mathrm{Cm} \mathrm{H}_{2} \mathrm{O}\right) \\
\end{array}$ & $\begin{array}{c}\text { Soll } \\
\text { Molsture } \\
\text { Tension } \\
\left(\mathrm{cm} \mathrm{H}_{2} \mathrm{O}\right)\end{array}$ & $\begin{array}{c}\text { Soll } \\
\text { Moisture } \\
\text { Tension } \\
\left(\mathrm{Cm} \mathrm{H}_{2} \mathrm{O}\right)\end{array}$ & $\begin{array}{c}\text { Soll } \\
\text { Molsture } \\
\text { Tension } \\
\left(\mathrm{Cm} \mathrm{H}_{2} \mathrm{O}\right)\end{array}$ & $\begin{array}{c}\text { Soll } \\
\text { Moisture } \\
\text { Tension } \\
\left(\mathrm{Cm} \mathrm{H}_{2} \mathrm{O}\right)\end{array}$ & 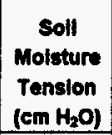 & $\begin{array}{c}\text { Soll } \\
\text { Molsture } \\
\text { Tonsion } \\
\left(\mathrm{Cm} \mathrm{H}_{2} \mathrm{O}\right)\end{array}$ & $\begin{array}{c}\text { Soll } \\
\text { Moisture } \\
\text { Tension } \\
\left(\mathrm{Cm} \mathrm{H}_{2} \mathrm{O}\right)\end{array}$ & 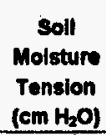 & $\begin{array}{c}\text { Soll } \\
\text { Molsture } \\
\text { Tonsion } \\
\left(\mathrm{Cm} \mathrm{H}_{2} \mathrm{O}\right) \\
\end{array}$ & $\begin{array}{c}\text { Soll } \\
\text { Molature } \\
\text { Tenslon } \\
\left(\mathrm{Cm} \mathrm{H}_{2} \mathrm{O}\right)\end{array}$ & $\begin{array}{c}\text { soll } \\
\text { Molisture } \\
\text { Tension } \\
\left(\mathrm{cm} \mathrm{H}_{2} \mathrm{O}\right) \\
\end{array}$ & $\begin{array}{c}\text { Soll } \\
\text { Moisture } \\
\text { Tension } \\
\left(\mathrm{Cm} \mathrm{H}_{2} \mathrm{O}\right)\end{array}$ & $\begin{array}{c}\text { soll } \\
\text { Moisture } \\
\text { Tension } \\
\left(\mathrm{em} \mathrm{H} \mathrm{H}_{2} \mathrm{O}\right)\end{array}$ & $\begin{array}{c}\text { Soll } \\
\text { Molsture } \\
\text { Tension } \\
\left(\mathrm{Cm} \mathrm{H} \mathrm{H}_{2} \mathrm{O}\right)\end{array}$ \\
\hline Date & Time & Days ${ }^{1}$ & $C 1^{\prime \prime}$ & $B 1^{a}$ & $A 1^{\prime \prime}$ & $\mathrm{C2}^{\mathrm{b}}$ & $B 2^{b}$ & $A 2^{b}$ & $\mathrm{CB}^{\mathrm{C}}$ & $\mathrm{B3}^{\mathrm{C}}$ & $A 3^{\mathrm{C}}$ & $C 4^{d}$ & $84^{d}$ & $\mathbf{A 4}^{d}$ & $c 5^{\circ}$ & $85^{\circ}$ & $A 5^{\circ}$ & $\mathrm{Cb}^{\prime}$ & $\mathrm{B6}^{\prime}$ & $A 6^{\prime}$ \\
\hline 424 & 3:10 & 0.716 & 75.9 & 70.3 & 71.2 & 53.4 & 51.9 & 51.7 & 41.6 & 35.8 & & 21.5 & 8.2 & 18.0 & 35.2 & 45.5 & 34.9 & 68.6 & -32.3 & .71 .58 \\
\hline 42415 & $3: 20$ & 0.722 & 76.8 & 70.3 & 71.2 & 53.4 & 52.8 & 52.6 & 41.6 & 36.7 & & 21.5 & 9.1 & 16.9 & 35.2 & 45.5 & 35.8 & 68.6 & -33.2 & .71 .68 \\
\hline $414 \%$ & $3: 30$ & 0.729 & 76.8 & 70.3 & 71.2 & 55.1 & 52.8 & 54.3 & 41.6 & 35.8 & & 22.4 & 7.4 & 17.7 & 35.2 & 46.4 & 35.8 & 69.5 & -31.4 & $\begin{array}{r}-71.58 \\
\end{array}$ \\
\hline $81347 \%$ & $3: 40$ & 0.736 & 77.7 & 71.1 & 71.2 & 54.2 & 51.1 & 52.6 & 41.6 & 37.6 & & 22.4 & 8.2 & 17.7 & 36.1 & 43.8 & 35.8 & 68.6 & .32 .3 & .71 .58 \\
\hline $82417 \%$ & $3: 50$ & 0.743 & 77.7 & 71.1 & 71.2 & 56.0 & 53.7 & 54.3 & 42.4 & 38.5 & & 24.1 & 8.1 & 19.5 & 36.1 & 45.5 & 36.7 & 68.6 & .31 .4 & -71.58 \\
\hline $62179 \%$ & $4: 00$ & 0.750 & 78.5 & 72.0 & 71.2 & 55.1 & 53.7 & 54.3 & 40.7 & 37.6 & & 22.4 & 8.1 & 19.5 & 35.2 & 44.7 & 35.8 & 69.5 & -31.4 & .71 .68 \\
\hline $14 \%$ & 4:10 & 0.757 & 79.4 & 71.1 & 71.2 & 56.0 & 53.7 & 55.2 & 42.4 & 38.5 & & 23.2 & 10.0 & 18.6 & 36.1 & 45.5 & 37.6 & 69.5 & -31.4 & .70 .78 \\
\hline 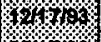 & $4: 20$ & 0.764 & 78.5 & 72.0 & 71.2 & 56.0 & 53.7 & 55.2 & 42.4 & 39.3 & & 24.1 & 8.1 & 19.5 & 35.2 & 43.8 & 37.6 & 69.5 & .32 .3 & .72 .48 \\
\hline 6171703 & $4: 30$ & 0.771 & 79.4 & 72.0 & 72.1 & 55.1 & 54.5 & 55.2 & 42.4 & 38.5 & & 25.0 & 10.8 & 19.5 & 36.1 & 45.5 & 35.8 & 69.5 & -30.5 & .71 .58 \\
\hline $421 \%$ & $4: 40$ & 0.778 & 79.4 & 72.0 & 72.9 & 56.0 & 55.4 & 55.2 & 43.3 & 38.5 & & 25.0 & 10.0 & 19.5 & 36.1 & 45.6 & 37.6 & 69.5 & -31.4 & .72 .48 \\
\hline $101 \%$ & $4: 50$ & 0.785 & 78.5 & 72.9 & 73.8 & 56.0 & 54.5 & 56.1 & 43.3 & 39.4 & & 25.0 & 10.8 & 19.5 & 36.1 & 45.5 & 36.7 & 69.5 & -30.5 & .72 .48 \\
\hline $814 \%$ & 5:00 & 0.792 & 78.4 & 72.0 & 73.8 & 56.0 & 54.5 & 55.2 & 43.3 & 39.4 & & 25.0 & 11.7 & 19.5 & 36.1 & 45.6 & 37.6 & 70.4 & -31.4 & .71 .58 \\
\hline 17470 & $5: 10$ & 0.799 & 79.4 & 72.9 & 72.1 & 56.0 & 55.4 & 54.3 & 44.2 & 40.2 & & 25.0 & 11.7 & 20.4 & 37.8 & 45.6 & 38.4 & 69.5 & -31.4 & .71 .58 \\
\hline $22430 \%$ & $5: 20$ & 0.806 & 79.4 & 72.9 & 73.8 & 56.9 & 56.3 & 56.1 & 44.2 & 39.4 & & 25.0 & 12.8 & 21.2 & 37.8 & 45.8 & 37.6 & 72.2 & -31.4 & .7248 \\
\hline $6214 \%$ & $5: 30$ & 0.813 & 79.4 & 72.9 & 73.8 & 56.9 & 56.3 & 58.7 & 44.2 & 40.2 & & 25.0 & 12.6 & 22.1 & 38.7 & 47.3 & 38.4 & 71.3 & -30.5 & .71 .58 \\
\hline 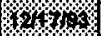 & $5: 40$ & 0.819 & 79.4 & 73.8 & 74.7 & 58.6 & 56.3 & 56.1 & 45.1 & 40.2 & & 25.9 & 12.6 & 21.2 & 39.6 & 46.4 & 38.4 & 72.2 & .29 .7 & .71 .58 \\
\hline $8 \%$ & 5.50 & 0.826 & 79.4 & 73.8 & 75.6 & 57.7 & 57.1 & 56.8 & 43.3 & 41.1 & & 25.9 & 12.6 & 23.0 & 39.8 & 45.8 & 38.4 & 72.2 & .30 .5 & .71 .58 \\
\hline $141 \%$ & $6: 00$ & 0.833 & 80.3 & 74.6 & 74.7 & 58.6 & 56.3 & 58.7 & 45.1 & 41.1 & & 26.8 & 12.6 & 23.0 & 38.7 & 47.3 & 38.4 & 71.3 & -30.5 & .72 .48 \\
\hline $601 \% 14$ & $6: 10$ & 0.840 & 89.1 & 74.6 & 74.7 & 57.7 & 57.1 & 58.7 & 45.1 & 41.1 & & 27.6 & 13.5 & 23.0 & 38.7 & 49.8 & 37.8 & 71.3 & -29.7 & .71 .58 \\
\hline 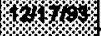 & $6: 20$ & 0.847 & 81.1 & 74.6 & 74.7 & 58.6 & 57.1 & 58.7 & 45.9 & 42.0 & & 26.8 & 13.5 & 23.0 & 38.7 & 49.0 & 39.3 & 73.0 & .29 .7 & .72 .48 \\
\hline $1 \% 179$ & 6:30 & 0.854 & 80.3 & 74.6 & 74.7 & $\infty 0.4$ & 56.3 & 58.7 & 45.9 & 42.0 & & 27.6 & 13.5 & 23.0 & 39.6 & 49.1 & 40.2 & 73.0 & .29 .7 & .73 .38 \\
\hline 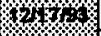 & $6: 40$ & 0.861 & 82.0 & 74.6 & 74.7 & 59.5 & 58.0 & 58.7 & 45.9 & 42.0 & & 27.6 & 14.3 & 23.0 & 39.8 & 48.2 & 40.2 & 73.1 & -29.7 & .72 .48 \\
\hline 82474 & $6: 50$ & 0.868 & 81.2 & 72.9 & 74.7 & 60.4 & 58.8 & 57.8 & 45.9 & 43.8 & & 28.5 & 14.3 & 23.0 & 39.6 & 49.1 & 39.3 & 72.2 & .29 .7 & .72 .48 \\
\hline $8 \% 1402$ & $7: 00$ & 0.876 & 81.2 & 74.6 & 74.7 & 59.5 & 58.8 & 58.7 & 45.9 & 42.8 & & 28.5 & 14.3 & 24.8 & 40.5 & 49.1 & 40.2 & 72.2 & -30.5 & .70 .78 \\
\hline $81 \% \%$ & $7: 10$ & 0.882 & 82.0 & 73.8 & 75.6 & 59.5 & 57.1 & 59.6 & 47.7 & 43.8 & & 27.6 & 13.5 & 23.9 & 39.8 & 49.1 & 39.3 & 72.2 & .29 .7 & -72.48 \\
\hline 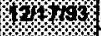 & $7: 20$ & 0.899 & 82.9 & 74.6 & 75.8 & 59.5 & 58.9 & 58.7 & 47.7 & 43.8 & & 28.5 & 15.2 & 23.9 & 39.6 & 49.1 & 41.0 & 73.1 & .29 .6 & $\begin{array}{r}-73.28 \\
\end{array}$ \\
\hline $1 \% \%$ & $7: 30$ & 0.896 & 82.9 & 75.5 & 76.4 & 59.5 & 59.7 & 59.6 & 47.7 & 43.8 & & 28.5 & 16.1 & 23.9 & 40.5 & 48.2 & 41.0 & 72.2 & -29.6 & -72.48 \\
\hline (124\% & $7: 40$ & 0.903 & 82.9 & 75.5 & 75.6 & 59.5 & 58.9 & 60.4 & 48.5 & 43.8 & & 28.5 & 16.1 & 23.0 & 39.6 & 48.1 & 41.9 & 73.1 & -30.5 & $\begin{array}{r}.73 .28 \\
\end{array}$ \\
\hline 14476 & $7: 50$ & 0.910 & 82.9 & 76.4 & 75.6 & 60.4 & 58.9 & 61.3 & 48.5 & 44.7 & & 28.5 & 16.1 & 24.8 & 39.6 & 48.1 & 41.0 & 73.1 & -29.6 & -75.08 \\
\hline 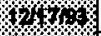 & $8: 00$ & 0.917 & 82.9 & 75.5 & 76.4 & 58.6 & 60.6 & 61.3 & 48.5 & 44.7 & & 28.5 & 16.1 & 28.3 & 39.6 & 49.1 & 41.0 & 73.1 & .29 .6 & -75.08 \\
\hline $1201 \%$ & $8: 10$ & 0.924 & 82.9 & 77.2 & 77.3 & 60.4 & 60.6 & 62.2 & 49.4 & 44.7 & & 28.5 & 16.1 & 26.5 & 39.6 & 49.1 & 41.8 & 73.1 & -29.7 & -75.08 \\
\hline 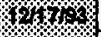 & $8: 20$ & 0.831 & 82.8 & 76.4 & 78.2 & 62.1 & 60.6 & 61.3 & 49.4 & 44.7 & & 27.6 & 18.7 & 28.5 & 39.6 & 49.8 & 41.0 & 73.1 & -29.7 & -75.08 \\
\hline 610120 & $8: 30$ & 0.938 & 82.9 & 78.1 & 78.2 & 61.2 & 61.4 & 62.2 & 49.4 & 45.5 & & 28.5 & 18.7 & 26.5 & 39.6 & 49.0 & 41.0 & 72.2 & -29.7 & .74 .18 \\
\hline $4 \% 179$ & $8: 40$ & 0.944 & 82.9 & 77.2 & 78.2 & 61.2 & 59.7 & 63.9 & 49.4 & 45.5 & & 28.5 & 17.8 & 26.5 & 39.6 & 51.7 & 41.8 & 73.0 & -29.7 & .75 .08 \\
\hline$\% y$ & $8: 50$ & 0.951 & 82.8 & 77.2 & 78.2 & 62.1 & 61.4 & 62.2 & 50.2 & 46.4 & & 26.7 & 18.9 & 27.4 & 39.6 & 51.6 & 41.8 & 72.1 & -30.5 & .75 .08 \\
\hline 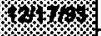 & $9: 00$ & 0.058 & 82.9 & 78.1 & 78.2 & 62.8 & 62.3 & 62.2 & 51.1 & 45.5 & & 27.6 & 19.5 & 27.4 & 40.4 & 50.8 & 41.8 & 72.1 & -29.7 & .75 .08 \\
\hline 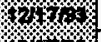 & 8:10 & 0.005 & 84.6 & 78.9 & 79.8 & 62.8 & 62.3 & 63.0 & 52.0 & 46.4 & & 28.5 & 19.5 & 27.4 & 42.1 & 51.6 & 43.6 & 73.0 & -29.7 & .75 .08 \\
\hline 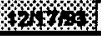 & $8: 20$ & 0.972 & 84.6 & 78.8 & 80.8 & 63.8 & 03.1 & 63.9 & 51.2 & 47.3 & & 28.5 & 20.4 & 27.4 & 42.1 & 52.5 & 44.4 & 75.6 & -29.7 & .75 .98 \\
\hline
\end{tabular}


Table 5. Tensiometer Data

\begin{tabular}{|c|c|c|c|c|c|c|c|c|c|c|c|c|c|c|c|c|c|c|c|c|}
\hline & & & & & & & & & Ten & siometer I & Data & & & & & & & & & \\
\hline & & Test & $\begin{array}{c}\text { Soil } \\
\text { Molsture } \\
\text { Tension } \\
\left(\mathrm{cm} \mathrm{H}_{2} \mathrm{O}\right) \\
\end{array}$ & $\begin{array}{c}\text { Soll } \\
\text { Molsture } \\
\text { Tension } \\
\left(\mathrm{Cm} \mathrm{H}_{2} \mathrm{O}\right) \\
\end{array}$ & $\begin{array}{c}\text { Soll } \\
\text { Moisture } \\
\text { Tension } \\
\left(\mathrm{cm} \mathrm{H}_{2} \mathrm{O}\right) \\
\end{array}$ & 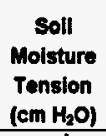 & $\begin{array}{c}\text { Soll } \\
\text { Molsture } \\
\text { Tension } \\
\text { (cm } \mathrm{H}_{2} \text { O) } \\
\end{array}$ & \begin{tabular}{|c} 
Soll \\
Moisture \\
Tension \\
(cm $\left.\mathrm{H}_{2} \mathrm{O}\right)$ \\
\end{tabular} & 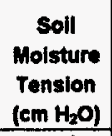 & $\begin{array}{c}\text { Soll } \\
\text { Molsture } \\
\text { Tension } \\
\left(\mathrm{Cm} \mathrm{H}_{2} \mathrm{O}\right) \\
\end{array}$ & $\begin{array}{c}\text { Soil } \\
\text { Moisture } \\
\text { Tension } \\
\left(\mathrm{Cm} \mathrm{H} \mathrm{H}_{2} \mathrm{O}\right) \\
\end{array}$ & $\begin{array}{c}\text { Soll } \\
\text { Moisture } \\
\text { Tension } \\
\text { (cm HzO) }\end{array}$ & $\begin{array}{c}\text { soll } \\
\text { Molsture } \\
\text { Tension } \\
\left(\mathrm{cm} \mathrm{H}_{2} \mathrm{O}\right)\end{array}$ & $\begin{array}{c}\text { Soil } \\
\text { Moisture } \\
\text { Tension } \\
\left(\mathrm{Cm} \mathrm{H}_{2} \mathrm{O}\right) \\
\end{array}$ & $\begin{array}{c}\text { Soll } \\
\text { Moisture } \\
\text { Tonsion } \\
\left(\mathrm{Cm} \mathrm{H}_{2} \mathrm{O}\right) \\
\end{array}$ & $\begin{array}{c}\text { Soll } \\
\text { Molsture } \\
\text { Tension } \\
\left.\text { (em } \mathrm{H}_{2} \mathrm{O}\right) \\
\end{array}$ & $\begin{array}{c}\text { Soll } \\
\text { Moisture } \\
\text { Tension } \\
\left(\mathrm{cm} \mathrm{H}_{2} \mathrm{O}\right)\end{array}$ & $\begin{array}{c}\text { Soll } \\
\text { Molsture } \\
\text { Tension } \\
\left.\text { (em } \mathrm{H}_{2} \mathrm{O}\right) \\
\end{array}$ & $\begin{array}{c}\text { soll } \\
\text { Molsture } \\
\text { Tenslon } \\
\left(\mathrm{em} \mathrm{H}_{2} \mathrm{O}\right) \\
\end{array}$ & $\begin{array}{c}\text { Soll } \\
\text { Molsture } \\
\text { Tension } \\
\text { (em H2O) }\end{array}$ \\
\hline Date & Time & Days' & $C 1^{\prime \prime}$ & $B 1^{\prime \prime}$ & $A 1^{\prime \prime}$ & $\mathrm{C2}^{\mathrm{b}}$ & $\mathrm{B2}^{\mathrm{b}}$ & $A 2^{b}$ & $\mathrm{C3}^{\mathrm{C}}$ & $\mathrm{B3}^{\mathrm{C}}$ & $\mathbf{A 3}^{\mathbf{C}}$ & $\mathrm{C}^{\mathrm{d}}$ & $\mathrm{BA}^{\mathrm{d}}$ & $A 4^{d}$ & $C 5^{\circ}$ & $B 5^{\circ}$ & $A 5^{\circ}$ & $c 6^{\prime}$ & $86^{\prime}$ & $A 6^{\prime}$ \\
\hline $1 \%$ & $9: 30$ & 0.979 & 84.6 & 78.9 & 80.8 & 63.8 & 63.1 & 63.8 & 51.8 & 48.2 & & 29.3 & 20.3 & 28.2 & 40.4 & 51.6 & 44.4 & 74.7 & -29.7 & -75.08 \\
\hline $101 \%$ & \begin{tabular}{|l}
$9: 40$ \\
\end{tabular} & 0.080 & 84.8 & 78.9 & 80.7 & 63.8 & 63.1 & 64.8 & 51.9 & 49.9 & & 28.5 & 21.2 & 30.0 & 40.4 & 52.5 & 44.4 & 73.8 & .29 .7 & .75 .08 \\
\hline 6016 & \begin{tabular}{|l|}
$9: 50$ \\
\end{tabular} & 0.003 & 86.3 & 78.9 & 80.7 & 64.6 & 64.0 & 65.6 & 51.8 & 49.9 & & 28.5 & 20.3 & 30.0 & 42.1 & 53.3 & 44.4 & 72.9 & -29.7 & .76 .88 \\
\hline $121 \%$ & 10:00 & 1.000 & 85.4 & 79.8 & 80.7 & 64.6 & 65.7 & 65.6 & 52.8 & 49.9 & & 28.5 & 22.0 & 30.0 & 42.1 & 53.3 & 44.4 & 72.8 & .29 .7 & .75 .98 \\
\hline$\% 2 \%$ & 10:10 & 1.007 & 86.3 & 80.6 & 82.5 & 64.6 & 64.8 & 65.6 & 52.8 & 50.8 & & 28.4 & 22.9 & 30.0 & 41.2 & 53.3 & 44.4 & 73.8 & -29.8 & .76 .88 \\
\hline 12810 & $10: 20$ & 1.014 & 86.3 & 81.5 & 82.5 & 84.6 & 65.7 & 66.5 & 54.5 & 51.7 & & 29.3 & 23.8 & 30.8 & 42.0 & 54.1 & 44.3 & 73.7 & -29.8 & .76 .88 \\
\hline 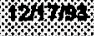 & 10:30 & 1.021 & 86.3 & 80.6 & 83.3 & 84.6 & 66.5 & 66.5 & 54.5 & 51.7 & & 28.4 & 24.6 & 30.8 & 42.0 & 54.1 & 46.1 & 73.7 & -29.8 & -76.88 \\
\hline $1 \%$ & $10: 40$ & 1.028 & 87.1 & 81.5 & 82.4 & 65.5 & 66.5 & 67.4 & 55.3 & 51.7 & & 29.3 & 24.6 & 30.8 & 42.0 & 54.1 & 46.0 & 73.7 & -29.8 & .76 .88 \\
\hline$\%$ & $10: 50$ & 1.038 & 87.1 & 82.3 & 83.3 & 65.4 & 66.5 & 67.4 & 56.2 & 51.7 & & 29.3 & 23.7 & 31.7 & 42.0 & 54.1 & 46.0 & 72.8 & .29 .8 & .76 .88 \\
\hline $1411 \%$ & $11: 00$ & 1.042 & 88.0 & 82.3 & 83.3 & 87.2 & 66.5 & 86.5 & 55.3 & 52.5 & & 30.2 & 23.7 & 32.6 & 42.0 & 55.0 & 46.0 & 73.7 & -29.8 & .76 .88 \\
\hline $101 \%$ & 11:10 & 1.049 & 88.0 & 82.3 & 84.2 & 66.3 & 66.5 & 69.1 & 56.2 & 51.7 & & 31.0 & 25.5 & 31.7 & 42.0 & 55.8 & 46.0 & 74.5 & -29.8 & .76 .88 \\
\hline $10 \%$ & $11: 20$ & 1.056 & 88.0 & 82.3 & 84.2 & 87.2 & 67.3 & 88.2 & 57.8 & 52.5 & & 31.0 & 25.5 & 32.6 & 42.0 & 54.1 & 46.9 & 75.4 & -28.9 & .76 .88 \\
\hline $121 \%$ & $11: 30$ & 1.063 & 88.8 & 83.2 & 84.2 & 67.2 & 67.3 & 69.1 & 56.2 & 53.4 & & 30.2 & 25.5 & 33.4 & 42.0 & 54.1 & 48.9 & 75.4 & -29.8 & .78 .58 \\
\hline 1021701 & 11:40 & 1.000 & 88.8 & 83.2 & 84.2 & 67.2 & 68.2 & 69.1 & 56.2 & 53.4 & & 30.2 & 26.3 & 33.4 & 42.8 & 55.8 & 46.9 & 74.5 & -29.8 & -78.58 \\
\hline 121414 & $11: 50$ & 1.070 & 88.8 & 83.2 & 85.0 & 86.3 & 67.3 & 69.1 & 57.0 & 53.4 & & 29.3 & 26.3 & 33.4 & 42.8 & 55.8 & 46.9 & 74.5 & -29.8 & -78.58 \\
\hline $1211 \%$ & $12: 00$ & 1.083 & 88.8 & 83.2 & 85.0 & 67.2 & 68.2 & 69.1 & 57.0 & 53.4 & & 30.2 & 28.3 & 33.4 & 42.8 & 54.9 & 47.7 & 74.5 & .29 .8 & .79 .48 \\
\hline $1 \% 1 \%$ & 12:10 & 1.090 & 88.8 & 84.0 & 85.9 & 68.0 & 68.2 & 69.1 & 57.0 & 54.3 & & 31.0 & 28.3 & 33.4 & 42.8 & 55.8 & 48.6 & 74.5 & -29.8 & .78 .68 \\
\hline $10110 \%$ & $12: 20$ & 1.097 & 89.7 & 84.0 & 85.0 & 68.0 & 69.0 & 69.1 & 57.9 & 53.4 & & 31.8 & 26.3 & 33.4 & 42.8 & 55.8 & 47.7 & 75.4 & .29 .8 & .78 .68 \\
\hline $101 \%$ & $12: 30$ & 1.104 & 89.7 & 84.0 & 85.8 & 68.9 & 69.0 & 69.8 & 57.9 & 54.3 & & 31.8 & 27.2 & 33.4 & 42.8 & 55.8 & 48.5 & 75.4 & -29.9 & .79 .48 \\
\hline $121 \%$ & $12: 40$ & 1.111 & 89.7 & 84.0 & 86.7 & 68.9 & 69.9 & 71.7 & 59.6 & 55.2 & & 32.8 & 27.2 & 34.3 & 42.8 & 55.8 & 48.5 & 76.2 & -29.8 & -80.38 \\
\hline $12 \%$ & $\begin{array}{l}12: 50 \\
\end{array}$ & 1.118 & 89.7 & 84.0 & 85.8 & 68.9 & 69.9 & 72.6 & 59.6 & 58.0 & & 33.6 & 27.1 & 34.3 & 42.8 & 55.7 & 48.5 & 76.2 & -29.8 & -79.48 \\
\hline 102170 & $13: 00$ & 1.126 & 89.7 & 84.9 & 85.9 & 68.9 & 69.9 & 72.6 & 58.6 & 56.0 & & 33.6 & 27.1 & 34.3 & 43.6 & 56.6 & 47.7 & 76.2 & -29.8 & -80.38 \\
\hline 120174 & 13:10 & 1.132 & 89.7 & 84.9 & 85.9 & 68.9 & 69.9 & 72.5 & 59.6 & 58.0 & & 33.6 & 28.0 & 35.2 & 42.8 & 55.7 & 48.5 & $\pi 7.0$ & .29 .8 & -80.38 \\
\hline $1 \% 14 \%$ & $13: 20$ & 1.130 & 89.7 & 84.9 & 87.8 & 69.7 & 70.7 & 72.5 & 59.6 & 58.9 & & 35.4 & 27.1 & 35.1 & 43.6 & 55.7 & 48.5 & 76.2 & .29 .0 & -80.38 \\
\hline 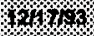 & $13: 30$ & 1.140 & 80.5 & 84.9 & 87.6 & 68.8 & 69.8 & 71.7 & 59.5 & 56.8 & & 33.6 & 28.0 & 35.1 & 44.5 & 58.6 & 49.3 & 78.1 & -29.9 & -80.38 \\
\hline $101 \% 3$ & $13: 40$ & 1.153 & 80.5 & 84.8 & 86.7 & 69.7 & 69.8 & 72.5 & 60.4 & 57.8 & & 35.3 & 28.8 & 36.0 & 44.4 & 58.6 & 48.5 & 77.9 & .29 .0 & -80.38 \\
\hline $13 \% 1 \%$ & $13: 50$ & 1.100 & 89.6 & 85.7 & 86.7 & 70.6 & 69.8 & 72.5 & 60.4 & 56.9 & & 35.3 & 28.8 & 36.0 & 44.4 & 58.5 & 49.3 & 77.9 & -28.2 & -80.38 \\
\hline 12474 & $14: 00$ & 1.167 & 90.5 & 85.7 & 87.6 & 70.5 & 69.8 & 72.5 & 81.2 & 56.9 & & 35.3 & 27.8 & 36.9 & 45.3 & 56.5 & 48.4 & 77.8 & -28.2 & -81.28 \\
\hline 12474 & \begin{tabular}{|l|}
$14: 10$ \\
\end{tabular} & 1.174 & 80.5 & 85.7 & 87.6 & 70.5 & 70.7 & 72.5 & 61.2 & 56.9 & & 35.3 & 27.8 & 36.9 & 45.3 & 57.4 & 48.4 & 77.8 & -29.1 & -81.28 \\
\hline $10 \% 401$ & 14:20 & 1.181 & 91.3 & 85.7 & 87.5 & 70.5 & 70.7 & 72.5 & 61.2 & 56.9 & & 35.3 & 28.8 & 37.7 & 45.3 & 57.4 & 50.1 & 78.7 & -29.1 & -81.28 \\
\hline $1 \% 4$ & \begin{tabular}{|l|l}
$14: 30$ \\
\end{tabular} & 1.188 & 01.3 & 85.7 & 87.5 & 71.4 & 70.7 & 73.4 & 62.1 & 59.5 & & 37.1 & 28.8 & 36.9 & 45.2 & 58.2 & 50.1 & 78.7 & -29.1 & -80.48 \\
\hline 141419 & $14: 40$ & 1.194 & 91.3 & 85.7 & 87.5 & 70.5 & 69.8 & 74.2 & 62.1 & 59.5 & & 38.8 & 28.8 & 34.2 & 46.1 & 58.2 & 51.0 & 78.6 & -28.2 & -80.48 \\
\hline 12118 & $14: 50$ & 1.201 & 80.4 & 85.7 & 88.6 & 70.5 & 69.8 & 74.2 & 61.2 & 56.8 & 60.4 & 38.8 & 27.9 & 35.1 & 45.2 & 57.3 & 50.1 & 79.5 & -29.1 & -80.48 \\
\hline $15 \% \%$ & \begin{tabular}{|l|}
$15: 00$ \\
\end{tabular} & 1.208 & 01.3 & 85.6 & 86.6 & 70.5 & 71.5 & 74.2 & 61.2 & 56.8 & 80.4 & 38.8 & 29.6 & 35.1 & 46.9 & 57.3 & 49.2 & 78.8 & -28.3 & 80.48 \\
\hline $14 \%$ & 15:10 & 1.216 & 81.3 & 85.6 & 87.5 & 70.5 & 70.8 & 74.2 & 61.2 & 56.8 & 60.4 & 36.8 & 28.7 & 36.8 & 47.8 & 57.3 & 50.1 & 80.3 & -28.3 & -60.48 \\
\hline $124 \%$ & 15:20 & 1.222 & 81.3 & 85.6 & 87.5 & 70.5 & 70.6 & 74.2 & 82.0 & 56.8 & 60.4 & 38.8 & 29.6 & 37.7 & 47.8 & 57.3 & 50.1 & 79.5 & -28.3 & 80.48 \\
\hline 12471 & $15: 30$ & 1.220 & 91.3 & 85.6 & 87.5 & 70.5 & 70.8 & 74.2 & 82.0 & 56.8 & 60.4 & 36.8 & 28.7 & 36.8 & 47.8 & 57.3 & 50.9 & 80.3 & .28 .3 & $\$ 0.48$ \\
\hline 142474 & 15:40 & 1.236 & 91.3 & 84.8 & 86.6 & 70.5 & 71.5 & 74.2 & 82.0 & 58.8 & 60.4 & 38.8 & 27.9 & 36.8 & 47.8 & 57.3 & 49.2 & 80.3 & -28.3 & 80.48 \\
\hline
\end{tabular}


Table 5. Tensiometer Data

\begin{tabular}{|c|c|c|c|c|c|c|c|c|c|c|c|c|c|c|c|c|c|c|c|c|}
\hline & & & & & & & & & Ten & siometer I & Data & & & & & & & & & \\
\hline & & Test & $\begin{array}{c}\text { Soll } \\
\text { Molsture } \\
\text { Tension } \\
\left(\mathrm{em} \mathrm{H}_{2} \mathrm{O}\right) \\
\end{array}$ & 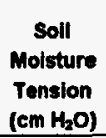 & 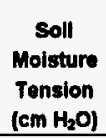 & $\begin{array}{c}\text { Soll } \\
\text { Moisture } \\
\text { Tenslon } \\
\left.\text { (em } \mathrm{H}_{2} \mathrm{O}\right) \\
\end{array}$ & $\begin{array}{c}\text { Soll } \\
\text { Molsture } \\
\text { Tension } \\
\text { (em } \mathrm{H}_{2} \mathrm{O} \text { ) } \\
\end{array}$ & 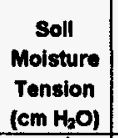 & $\begin{array}{c}\text { Soll } \\
\text { Molsture } \\
\text { Tension } \\
\left(\mathrm{Cm}_{2} \mathrm{O}\right) \\
\end{array}$ & 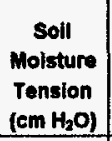 & 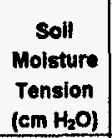 & $\begin{array}{c}\text { Soll } \\
\text { Moisture } \\
\text { Tension } \\
\left(\mathrm{Cm} \mathrm{H}_{2} \mathrm{O}\right) \\
\end{array}$ & $\begin{array}{c}\text { Soll } \\
\text { Moisture } \\
\text { Tension } \\
\left(\mathrm{Cm} \mathrm{H}_{2} \mathrm{O}\right)\end{array}$ & $\begin{array}{c}\text { Soil } \\
\text { Moisture } \\
\text { Tenslon } \\
\left(\mathrm{em} \mathrm{H}_{2} \mathrm{O}\right) \\
\end{array}$ & $\begin{array}{c}\text { Soll } \\
\text { Molsture } \\
\text { Tenslon } \\
\left(\mathrm{Cm} \mathrm{H}_{2} \mathrm{O}\right)\end{array}$ & $\begin{array}{c}\text { Soll } \\
\text { Moiature } \\
\text { Tenslon } \\
\left(\mathrm{Cm} \mathrm{H}_{2} \mathrm{O}\right) \\
\end{array}$ & $\begin{array}{c}\text { Soll } \\
\text { Moisture } \\
\text { Tenslon } \\
\left(\mathrm{cm} \mathrm{H}_{2} \mathrm{O}\right) \\
\end{array}$ & $\begin{array}{c}\text { Soll } \\
\text { Molsture } \\
\text { Tension } \\
\text { (cm } \mathrm{H}_{2} \mathrm{O} \text { ) } \\
\end{array}$ & $\begin{array}{c}\text { Soll } \\
\text { Molsture } \\
\text { Tonsion } \\
\left.\text { (cm } \mathrm{H}_{2} \mathrm{O}\right) \\
\end{array}$ & $\begin{array}{c}\text { Soll } \\
\text { Moisture } \\
\text { Tensilon } \\
\left(\mathrm{Cm} \mathrm{H}_{2} \mathrm{O}\right) \\
\end{array}$ \\
\hline Date & Time & Days' & $C 1^{\prime}$ & $B 1^{2}$ & $A 1^{*}$ & $\mathrm{C2}^{\mathrm{b}}$ & $\mathrm{B2}^{\mathrm{b}}$ & $A 2^{6}$ & $\mathrm{C3}^{\mathrm{C}}$ & $\mathrm{B}^{\mathrm{C}}$ & $A 3^{c}$ & $\mathrm{CA}^{\mathrm{d}}$ & $B 4^{d}$ & $\mathbf{A 4}^{\mathrm{d}}$ & $\cos ^{\circ}$ & $\mathrm{BF}^{\circ}$ & $\mathrm{As}^{\circ}$ & $66^{\prime}$ & $B 6^{\prime}$ & $A 6^{f}$ \\
\hline Y $1 \%$ & 15:50 & 1.243 & 91.3 & 85.6 & 87.5 & 70.5 & 72.3 & 74.2 & 61.1 & 58.6 & 60.4 & 38.8 & 29.6 & 37.7 & 47.7 & 57.3 & 50.0 & 81.2 & -28.3 & -60.48 \\
\hline $67 \%$ \% & $16: 00$ & 1.250 & 91.3 & 85.6 & 87.5 & 70.5 & 71.5 & 74.2 & 62.0 & 57.7 & 60.3 & 39.6 & 28.7 & 37.7 & 47.7 & 57.3 & 50.8 & 81.2 & -28.3 & -80.48 \\
\hline 1240 & 16:10 & 1.267 & 91.3 & 85.6 & 87.5 & 70.5 & 71.5 & 74.2 & 62.0 & 57.7 & 60.3 & 39.6 & 28.7 & 37.7 & 47.7 & 57.3 & 50.8 & 81.2 & -28.3 & 84.38 \\
\hline 123140 & $16: 20$ & 1.264 & 81.3 & 85.6 & 87.5 & 70.5 & 71.5 & 74.2 & 62.0 & 58.6 & 81.2 & 40.5 & 29.8 & 37.7 & 47.7 & 57.3 & 50.0 & 81.2 & .28 .3 & 82.18 \\
\hline 12747. & $16: 30$ & 1.271 & 91.3 & 85.6 & 87.5 & 69.6 & 71.5 & 73.3 & 62.0 & 59.5 & 62.1 & 41.4 & 29.6 & 37.7 & 47.7 & 54.7 & 50.0 & 81.2 & -28.3 & -81.38 \\
\hline $124+4$ & $16: 40$ & 1.278 & 91.3 & 85.6 & 88.6 & 70.5 & 71.5 & 73.3 & 62.0 & 58.6 & 61.2 & 41.4 & 29.6 & 37.7 & 48.6 & 57.3 & 50.0 & 81.2 & -28.3 & .81 .38 \\
\hline 12425 & $16: 50$ & 1.285 & 90.4 & 85.6 & 87.5 & 70.5 & 72.3 & 73.3 & 62.0 & 57.7 & 62.1 & 42.3 & 30.5 & 37.7 & 48.6 & 57.3 & 50.9 & 82.1 & -27.4 & .82 .18 \\
\hline 6row & $17: 00$ & 1.292 & 91.3 & 85.6 & 87.5 & 70.5 & 72.3 & 73.4 & 62.0 & 59.5 & 62.1 & 42.3 & 29.6 & 37.7 & 48.6 & 57.3 & 50.8 & 82.1 & -28.3 & -83.08 \\
\hline 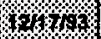 & \begin{tabular}{|l|}
$17: 10$ \\
\end{tabular} & 1.299 & 90.4 & 85.7 & 87.5 & 70.5 & 71.5 & 73.4 & 62.1 & 57.7 & 60.4 & 42.3 & 30.5 & 37.7 & 48.7 & 58.2 & 51.0 & 82.1 & -28.3 & 83.08 \\
\hline $10 \%, 13$ & \begin{tabular}{|l|}
$17: 20$ \\
\end{tabular} & 1.306 & 91.3 & 85.7 & 86.7 & 69.6 & 71.5 & 72.5 & 82.1 & 58.6 & 63.0 & 42.3 & 30.5 & 37.7 & 47.8 & 57.3 & 51.0 & 82.1 & -28.2 & -82.98 \\
\hline 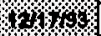 & $17: 30$ & 1.313 & 91.3 & 85.7 & 86.7 & 69.7 & 71.5 & 72.5 & 62.9 & 58.6 & 62.1 & 41.4 & 29.7 & 37.7 & 48.7 & 58.2 & 51.0 & 82.2 & -29.1 & -82.18 \\
\hline (24) & $17: 40$ & 1.319 & 90.5 & 85.7 & 87.6 & 70.5 & 72.4 & 725 & 62.1 & 57.7 & 82.1 & 42.3 & 31.4 & 37.7 & 48.7 & 58.3 & 51.0 & 82.2 & -28.2 & -82.88 \\
\hline $42178 \mathrm{~s}$ & $17: 50$ & 1.326 & 90.5 & 84.8 & 85.8 & 70.6 & 71.6 & 725 & 62.1 & 57.8 & 62.1 & 41.4 & 29.7 & 37.7 & 48.7 & 58.3 & 50.2 & 81.3 & -28.2 & -82.18 \\
\hline 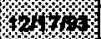 & $18: 00$ & 1.333 & 89.6 & 85.7 & 85.8 & 69.7 & 71.6 & 72.5 & 63.0 & 57.8 & 61.3 & 41.4 & 29.7 & 38.6 & 49.6 & 58.3 & 50.2 & 83.1 & -29.0 & -83.88 \\
\hline 12144 & $18: 10$ & 1.340 & 91.4 & 84.9 & 85.8 & 69.7 & 71.6 & 72.5 & 63.0 & 57.8 & 63.0 & 40.8 & 29.7 & 36.9 & 49.8 & 58.3 & 50.2 & 83.1 & -28.2 & -83.88 \\
\hline 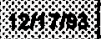 & $18: 20$ & 1.347 & 91.4 & 84.9 & 85.9 & 69.7 & 72.5 & 72.5 & 62.2 & 57.8 & 63.0 & 40.6 & 29.7 & 36.9 & 49.7 & 59.2 & 50.2 & 83.2 & -29.9 & -84.68 \\
\hline 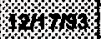 & $18: 30$ & 1.364 & 91.4 & 84.9 & 86.7 & 70.8 & 71.6 & 72.5 & 63.0 & 58.7 & 63.0 & 40.6 & 30.8 & 37.8 & 49.7 & 58.4 & 51.1 & 83.2 & -28.1 & -84.68 \\
\hline $13 \% 1 \% 3$ & $19: 00$ & 1.375 & 91.4 & 85.8 & 88.8 & 70.6 & 72.5 & 73.4 & 63.1 & 58.7 & 62.2 & 40.6 & 30.6 & 37.8 & 49.7 & 57.5 & 51.2 & 83.3 & -29.0 & -84.68 \\
\hline $101 \% 1 \%$ & 19:30 & 1.396 & 90.8 & 85.8 & 86.8 & 70.7 & 73.4 & 74.3 & 84.0 & 59.6 & 83.8 & 41.5 & 30.7 & 37.8 & 49.8 & 58.5 & 51.2 & 83.3 & -28.1 & -85.48 \\
\hline 12410 & 2000 & 1.417 & 91.5 & 85.8 & 88.8 & 70.7 & 73.4 & 74.3 & 64.8 & 59.6 & 64.0 & 42.4 & 30.7 & 38.7 & 49.8 & 58.5 & 51.2 & 82.5 & -28.9 & -80.38 \\
\hline $122178 \%$ & $20: 30$ & 1.438 & 91.5 & 85.8 & 86.8 & 70.7 & 73.4 & 74.3 & 65.8 & 80.5 & 84.0 & 42.4 & 30.7 & 39.8 & 50.7 & 58.5 & 51.3 & 84.2 & -28.9 & $-87: 18$ \\
\hline$y_{2} / 7,1$ & $21: 00$ & 1.458 & 91.5 & 85.8 & 87.7 & 70.7 & 74.3 & 75.2 & 64.8 & 60.5 & 64.0 & 42.4 & 31.6 & 39.6 & 50.7 & 58.5 & 50.4 & 84.3 & .28 .9 & -87.18 \\
\hline 2. & $21: 30$ & 1.479 & 91.5 & 85.8 & 87.7 & 72.5 & 73.4 & 74.4 & 65.8 & 59.6 & 84.9 & 41.6 & 32.5 & 39.6 & 51.6 & 59.4 & 51.3 & 85.2 & .29 .7 & 88.08 \\
\hline 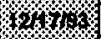 & $22: 00$ & 1.600 & 92.4 & 86.7 & 87.7 & 73.3 & 73.5 & 74.4 & 65.8 & 61.4 & 65.7 & 42.4 & 32.5 & 41.4 & 51.6 & 59.4 & 51.3 & 85.2 & -29.7 & .87 .98 \\
\hline 61414 & $22: 30$ & 1.621 & 92.4 & 87.6 & 87.7 & 72.5 & 74.3 & 76.1 & 65.8 & 60.5 & 68.6 & 42.4 & 32.5 & 40.5 & 51.6 & 59.4 & 52.2 & 85.2 & -28.8 & -88.88 \\
\hline 143.193 & $23: 00$ & 1.542 & 92.4 & 87.6 & 88.6 & 72.5 & 75.2 & 78.1 & 65.8 & 60.5 & 68.6 & 43.3 & 33.4 & 40.5 & 52.5 & 60.3 & 52.2 & 85.2 & -29.7 & -88.88 \\
\hline$(16 \% 1 \%$ & $23: 30$ & 1.563 & 93.3 & 87.6 & 87.7 & 73.4 & 75.2 & 76.1 & 66.7 & 80.5 & 68.8 & 43.3 & 34.3 & 41.4 & 51.6 & 59.5 & 52.2 & 86.1 & -29.7 & -88.88 \\
\hline 2348. & $0: 00$ & 1.693 & 93.3 & 86.7 & 88.8 & 71.8 & 75.2 & 78.1 & 67.5 & 61.4 & 87.5 & 43.3 & 34.3 & 40.5 & 52.5 & 59.5 & 53.1 & 86.1 & -28.7 & -81.48 \\
\hline 472109 & $0: 30$ & 1.004 & 93.3 & 88.5 & 88.6 & 74.2 & 76.1 & 77.0 & 67.6 & 81.4 & 67.5 & 42.5 & 34.3 & 41.4 & 52.5 & 59.5 & 52.2 & 88.1 & -29.7 & -91.48 \\
\hline 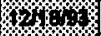 & $1: 00$ & 1.625 & 94.2 & 88.5 & 88.6 & 73.4 & 76.8 & 77.0 & 69.3 & 63.2 & 67.5 & 43.3 & 34.3 & 40.5 & 53.4 & 60.4 & 54.8 & 88.1 & -29.7 & -92.28 \\
\hline (1964) & $1: 30$ & 1.648 & 83.3 & 88.5 & 88.6 & 74.2 & 78.9 & 77.0 & 68.4 & 64.1 & 68.4 & 45.1 & 34.3 & 42.3 & 53.4 & 62.1 & 53.1 & 86.1 & -28.7 & -92.28 \\
\hline (12\% & $2: 00$ & 1.667 & 94.2 & 89.4 & 89.5 & 75.1 & 77.8 & 77.8 & 69.3 & 63.2 & 69.2 & 45.1 & 36.0 & 43.2 & 53.4 & 62.1 & 53.1 & 87.9 & -29.7 & .93 .18 \\
\hline 6 & $2: 30$ & 1.688 & 94.2 & 89.4 & 89.5 & 74.2 & 77.8 & 77.8 & 70.2 & 63.2 & 88.4 & 43.3 & 35.1 & 44.0 & 54.3 & 63.0 & 54.0 & 87.9 & -29.7 & .03 .18 \\
\hline 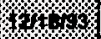 & $3: 00$ & 1.708 & 95.9 & 89.4 & 89.5 & 75.1 & 77.8 & 78.8 & 69.3 & 63.2 & 69.2 & 48.0 & 36.0 & 44.0 & 54.3 & 63.0 & 54.8 & 87.8 & .28 .8 & -03.18 \\
\hline (2721 & $3: 30$ & 1.729 & 95.0 & 89.4 & 89.5 & 75.1 & 77.8 & 79.6 & 70.2 & 63.2 & 70.1 & 46.0 & 36.0 & 44.0 & 54.3 & 63.0 & 54.8 & 87.8 & -29.7 & .93 .88 \\
\hline 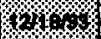 & $4: 00$ & 1.750 & 85.9 & 88.5 & 90.4 & 77.7 & 77.8 & 79.6 & 69.3 & 65.0 & 70.1 & 48.0 & 36.8 & 44.0 & 55.1 & 83.0 & 54.8 & 80.5 & -29.7 & .85 .78 \\
\hline 42410 & $4: 30$ & 1.771 & 95.9 & 89.4 & 91.2 & 76.9 & 78.7 & 79.6 & 70.2 & 64.1 & 71.0 & 46.8 & 37.7 & 44.0 & 54.3 & 63.0 & 54.8 & 88.8 & & \\
\hline (2) & $5: 00$ & 1.792 & 97.6 & 89.4 & 91.2 & 77.7 & 78.7 & 79.6 & 70.2 & 64.1 & 71.0 & 47.7 & 37.7 & 44.0 & 55.1 & 63.8 & 55.7 & 88.8 & & \\
\hline
\end{tabular}


Table 5. Tensiometer Data

\begin{tabular}{|c|c|c|c|c|c|c|c|c|c|c|c|c|c|c|c|c|c|c|c|c|}
\hline & & & & & & & & & Ten & siometer & Data & & & & & & & & & \\
\hline & & Test & 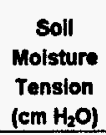 & $\begin{array}{c}\text { Soll } \\
\text { Molsture } \\
\text { Tension } \\
\left(\mathrm{cm} \mathrm{H}_{2} \mathrm{O}\right) \\
\end{array}$ & 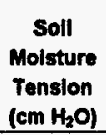 & 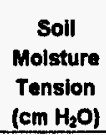 & 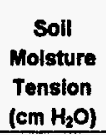 & $\begin{array}{c}\text { Soll } \\
\text { Moisture } \\
\text { Tension } \\
\left(\mathrm{Cm} \mathrm{H}_{2} \mathrm{O}\right) \\
\end{array}$ & $\begin{array}{c}\text { Soll } \\
\text { Molsture } \\
\text { Tension } \\
\left(\mathrm{Cm} \mathrm{H}_{2} \mathrm{O}\right) \\
\end{array}$ & \begin{tabular}{|c|} 
Soll \\
Molsture \\
Tension \\
(cm $\mathrm{H}_{2} \mathrm{O}$ ) \\
\end{tabular} & $\begin{array}{c}\text { Soil } \\
\text { Moisture } \\
\text { Tension } \\
\left(\mathrm{em} \mathrm{H}_{2} \mathrm{O}\right) \\
\end{array}$ & 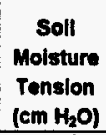 & $\begin{array}{c}\text { Soll } \\
\text { Molsture } \\
\text { Tension } \\
\left(\mathrm{Cm} \mathrm{H}_{2} \mathrm{O}\right) \\
\end{array}$ & $\begin{array}{c}\text { Soll } \\
\text { Molsture } \\
\text { Tension } \\
\text { (cm } \mathrm{H}_{2} \mathrm{O} \text { ) } \\
\end{array}$ & $\begin{array}{c}\text { Soll } \\
\text { Molsture } \\
\text { Tension } \\
\text { (em HzO) }\end{array}$ & $\begin{array}{c}\text { Soll } \\
\text { Moisture } \\
\text { Tension } \\
\left(\mathrm{Cm} \mathrm{H}_{2} \mathrm{O}\right) \\
\end{array}$ & $\begin{array}{c}\text { Soll } \\
\text { Moisture } \\
\text { Tension } \\
\left(\mathrm{em} \mathrm{H} \mathrm{H}_{2} \mathrm{O}\right) \\
\end{array}$ & $\begin{array}{c}\text { Soll } \\
\text { Molsture } \\
\text { Tanslon } \\
\left(\mathrm{em} \mathrm{H}_{2} \mathrm{O}\right) \\
\end{array}$ & 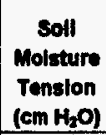 & $\begin{array}{c}\text { Soll } \\
\text { Molsture } \\
\text { Tension } \\
\left(\mathrm{Cm} \mathrm{H}_{2} \mathrm{O}\right) \\
\end{array}$ \\
\hline Date & Time & Days' & C1" & $B 1^{\prime \prime}$ & $A 1^{*}$ & $\mathrm{C2}^{\mathrm{b}}$ & $\mathrm{B2}^{\mathrm{b}}$ & $A 2^{\mathrm{b}}$ & $\mathrm{C3}^{\mathrm{c}}$ & $\mathrm{B3}^{\mathrm{C}}$ & $A 3^{C}$ & $\mathrm{C4}^{\mathrm{d}}$ & $B 4^{d}$ & $\mathrm{~A}^{\mathrm{d}}$ & $C 5^{\circ}$ & $B 5^{\circ}$ & $A 5^{\circ}$ & $c 6^{f}$ & $86^{\prime}$ & $A 6^{f}$ \\
\hline $121 \%$ & $5: 30$ & 1.813 & 95.9 & 92.0 & 00.4 & 76.9 & 78.7 & 79.6 & 70.2 & 65.0 & 71.0 & 46.0 & 37.7 & 44.9 & 55.1 & 63.0 & 56.5 & 90.5 & & \\
\hline $141 \%$ & $6: 00$ & 1.833 & 96.8 & 81.1 & 81.2 & 76.8 & 80.4 & 79.6 & 71.9 & 65.9 & 72.7 & 46.8 & 38.6 & 46.7 & 56.0 & 64.7 & 55.7 & 89.6 & & \\
\hline $1410 \%$ & 6:30 & 1.864 & 96.8 & 91.1 & 82.1 & 76.8 & 80.4 & 79.8 & 72.8 & 66.7 & 71.8 & 47.7 & 38.6 & 44.9 & 58.0 & 63.8 & 58.5 & 89.6 & & \\
\hline $121 \% 1$ & $7: 00$ & 1.875 & 96.8 & 91.1 & 92.1 & 77.7 & 80.4 & 80.5 & 72.8 & 68.7 & 71.8 & 46.8 & 39.5 & 45.8 & 55.1 & 64.7 & 56.5 & 89.6 & & \\
\hline $121 \%$ & $7: 30$ & 1.896 & 96.8 & 92.0 & 93.0 & 77.7 & 80.4 & 81.4 & 72.8 & 66.7 & 72.7 & 46.8 & 40.3 & 47.6 & 56.8 & 65.8 & 57.4 & 89.6 & & \\
\hline 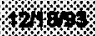 & $8: 00$ & 1.017 & 97.6 & 92.0 & 93.9 & 77.7 & 80.4 & 82.2 & 71.8 & 66.7 & 73.6 & 46.8 & 39.5 & 48.7 & 58.0 & 64.7 & 56.5 & 89.6 & & \\
\hline 1240 & $8: 30$ & 1.038 & 98.5 & 92.8 & 93.8 & 80.3 & 89.2 & 83.1 & 75.3 & 67.8 & 73.6 & 46.0 & 41.2 & 48.4 & 56.0 & 65.6 & 58.2 & 89.6 & & \\
\hline 1210 & $8: 00$ & 1.068 & 98.5 & 93.7 & 95.6 & 81.2 & 83.8 & 84.0 & 75.3 & 70.3 & 74.4 & 45.9 & 42.0 & 48.4 & 58.8 & 87.3 & 58.2 & 92.2 & & \\
\hline $140 \%$ & $9: 30$ & 1.979 & 98.5 & 93.6 & 94.7 & 81.1 & 83.8 & 84.8 & 78.2 & 70.2 & 74.4 & 46.8 & 42.0 & 48.4 & 57.6 & 67.3 & 59.1 & 00.4 & & \\
\hline $101 \%$ & $10: 00$ & 2.000 & 100.2 & 24.5 & 97.3 & 82.0 & 83.8 & 85.7 & 78.1 & 70.2 & 74.4 & 45.8 & 43.7 & 50.1 & 57.6 & 68.1 & 59.9 & 91.2 & & \\
\hline $401 \%$ & $10: 30$ & 2.021 & 101.0 & 95.3 & 97.3 & 82.8 & 85.5 & 86.8 & 78.1 & 70.2 & 75.2 & 45.0 & 44.6 & 51.0 & 56.7 & 68.1 & 59.9 & 80.3 & & \\
\hline 124000 & $11: 00$ & 2.042 & 101.8 & 96.2 & 97.2 & 83.7 & 86.3 & 86.5 & 77.8 & 73.7 & 76.1 & 49.4 & 44.6 & 51.0 & 58.4 & 68.9 & 60.7 & 938 & & \\
\hline 142140 & $11: 30$ & 2.063 & 100.1 & 95.3 & 97.2 & 83.7 & 85.4 & 86.5 & 78.9 & 72.0 & 75.2 & 46.8 & 44.5 & 51.0 & 56.7 & 69.8 & 60.7 & 91.2 & & \\
\hline $1826 \%$ & $12: 00$ & 2.083 & 102.7 & 97.0 & 99.0 & 83.7 & 87.1 & 87.4 & 77.8 & 72.8 & 77.0 & 46.8 & 46.3 & 52.7 & 60.1 & 69.7 & 62.4 & 91.1 & & \\
\hline $14640 \%$ & $12: 30$ & 2.104 & 102.7 & 96.1 & 98.9 & 84.5 & 87.1 & 87.4 & 79.5 & 74.8 & 77.8 & 49.4 & 46.2 & 51.8 & 60.1 & 69.7 & 62.3 & 92.8 & & \\
\hline 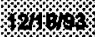 & $13: 00$ & 2.125 & 102.7 & 97.9 & 100.7 & 85.3 & 88.0 & 89.1 & 78.5 & 74.6 & 77.8 & 47.6 & 47.8 & 53.6 & 59.2 & 70.5 & 63.2 & 91.0 & & \\
\hline $1 \% 14 \%$ & $13: 30$ & 2.146 & 103.5 & 97.8 & 100.6 & 85.3 & 87.1 & 88.2 & 79.4 & 74.5 & 78.6 & 52.8 & 47.9 & 53.5 & 80.0 & 71.4 & 63.1 & 92.7 & & \\
\hline 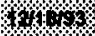 & $14: 00$ & 2.167 & 103.5 & 97.8 & 100.8 & 85.3 & 87.9 & 89.1 & 79.4 & 74.5 & 77.8 & 50.2 & 47.9 & 54.4 & 59.8 & 71.3 & 63.1 & 92.7 & & \\
\hline 14\% & $14: 30$ & 2.180 & 102.6 & 97.8 & 100.8 & 85.3 & 87.0 & 89.1 & 79.4 & 74.5 & 77.7 & 51.9 & 47.8 & 54.4 & 59.0 & 71.3 & 63.9 & 92.6 & & \\
\hline 1249 & $15: 00$ & 2.208 & 102.6 & 98.6 & 101.4 & 87.0 & 87.0 & 89.1 & 80.2 & 74.5 & 79.5 & 51.8 & 47.8 & 55.2 & 62.4 & 71.2 & 63.9 & 96.1 & & \\
\hline 12.01 & $15: 30$ & 2.229 & 101.7 & 98.6 & 100.6 & 85.2 & 87.9 & 88.2 & 79.3 & 74.5 & 78.6 & 49.3 & 47.8 & 55.2 & 60.7 & 69.5 & 62.1 & 95.2 & & \\
\hline $1821 \%$ & $16: 00$ & 2.250 & 103.4 & 97.8 & 99.7 & 88.1 & 87.0 & 89.9 & 80.2 & 74.5 & 79.5 & 50.1 & 47.8 & 54.4 & 60.7 & 70.4 & 63.0 & 96.1 & & \\
\hline $14 \% \%$ & $16: 30$ & 2.271 & 102.6 & 96.9 & 99.7 & 85.2 & 87.9 & 88.2 & 80.2 & 74.5 & 80.3 & 52.8 & 47.8 & 55.2 & 62.5 & 71.2 & 63.0 & 98.1 & & \\
\hline 121018 & $17: 00$ & 2.292 & 102.6 & 96.9 & 98.0 & 84.4 & 87.0 & 88.2 & 79.4 & 74.5 & 81.2 & 51.8 & 47.8 & 55.3 & 62.5 & 71.3 & 64.8 & 97.0 & & \\
\hline $4 \times 5 \times$ & $17: 30$ & 2.313 & 102.7 & 97.8 & 98.9 & 85.3 & 87.8 & 88.2 & 80.3 & 74.5 & 79.5 & 51.1 & 47.0 & 55.3 & 63.4 & 69.8 & 83.1 & 98.0 & & \\
\hline 141088 & $\begin{array}{l}18: 00 \\
\end{array}$ & 2.333 & 102.7 & 97.0 & 98.9 & 85.4 & 88.0 & 88.2 & 80.3 & 74.6 & 80.4 & 51.1 & 46.2 & 54.4 & 63.5 & 69.7 & 63.2 & 97.2 & & \\
\hline 1401 & $18: 30$ & 2.364 & 103.6 & 96.2 & 97.2 & 84.5 & 87.1 & 88.3 & 80.4 & 72.8 & 80.4 & 50.2 & 47.1 & 54.5 & 62.7 & 69.7 & 63.2 & 96.3 & & \\
\hline 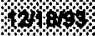 & $18: 00$ & 2.375 & 102.7 & 96.2 & 97.2 & 84.5 & 87.2 & 88.3 & 79.5 & 73.7 & 79.6 & 49.4 & 48.0 & 55.3 & 61.8 & 71.5 & 63.3 & 24.6 & & \\
\hline $124 \%$ & $19: 30$ & 2.396 & 102.8 & 96.2 & 98.1 & 84.6 & 87.2 & 88.3 & 79.6 & 74.6 & 80.4 & 49.4 & 48.0 & 55.4 & 62.7 & 71.5 & 63.3 & 08.4 & & \\
\hline $17218 \%$ & $20: 00$ & 2.417 & 102.8 & 96.2 & 98.1 & 84.6 & 87.2 & 88.3 & 79.6 & 73.7 & 81.3 & 51.1 & 47.2 & 55.4 & 62.7 & 70.7 & 65.0 & 97.3 & & \\
\hline 24848 & 20:30 & 2.438 & 102.8 & 96.2 & 98.1 & 84.6 & 87.2 & 88.3 & 79.6 & 74.6 & 81.3 & 50.3 & 48.1 & 55.4 & 62.8 & 71.6 & 65.1 & 97.4 & & \\
\hline $124 \%$ & $21: 00$ & 2.458 & 103.7 & 97.1 & 98.0 & 85.5 & 88.1 & 88.3 & 80.5 & 74.6 & 81.3 & 49.4 & 48.1 & 55.4 & 62.8 & 71.0 & 64.2 & 97.4 & & \\
\hline $12 \%$ & $21: 30$ & 2.479 & 103.7 & 98.2 & 98.2 & 86.3 & 87.2 & 88.3 & 80.5 & 74.6 & 81.3 & 51.2 & 49.8 & 55.4 & 62.8 & 71.6 & 65.1 & 99.2 & & \\
\hline $14 \%$ & 22:00 & 2.500 & 103.7 & 97.1 & 98.2 & 85.5 & 87.2 & 89.2 & 82.2 & 74.7 & 81.3 & 51.2 & 47.2 & 55.4 & 62.8 & 70.7 & 65.1 & 98.3 & & \\
\hline $13 \%$ & $22: 30$ & 2.521 & 103.7 & 96.3 & 98.2 & 86.4 & 87.3 & 89.2 & 81.4 & 74.7 & 81.4 & 50.3 & 48.1 & 55.4 & 62.8 & 21.6 & 65.1 & 100.1 & & \\
\hline $141 \% 1$ & $23: 00$ & 2.542 & 103.7 & 97.1 & 98.2 & 84.6 & 89.0 & 89.2 & 81.4 & 74.7 & 81.4 & 49.4 & 48.1 & 56.3 & 63.7 & 71.6 & 68.0 & 98.3 & & \\
\hline 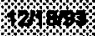 & $23: 30$ & 2563 & 104.6 & 97.1 & 98.2 & 87.2 & 88.1 & 89.2 & 82.3 & 74.7 & 81.4 & 50.3 & 48.1 & 56.3 & 63.7 & 71.6 & 65.1 & 98.3 & & \\
\hline 8 & $0: 00$ & 2.583 & 103.7 & 97.1 & 98.2 & 87.3 & 89.0 & 89.2 & 82.3 & 74.7 & 81.4 & 52.1 & 48.1 & 58.1 & 63.7 & 72.5 & 85.9 & 100.1 & & \\
\hline
\end{tabular}


Table 5. Tensiometer Data

\begin{tabular}{|c|c|c|c|c|c|c|c|c|c|c|c|c|c|c|c|c|c|c|c|c|}
\hline & & & & & & & & & Ten: & siometer & Data & & & & & & & & & \\
\hline & & Test & $\begin{array}{c}\text { Soll } \\
\text { Moisture } \\
\text { Tension } \\
\left.\text { (em } \mathrm{H}_{2} \mathrm{O}\right) \\
\end{array}$ & $\begin{array}{c}\text { Soll } \\
\text { Molsture } \\
\text { Tonslon } \\
\left.\text { (cm } \mathrm{H}_{2} \mathrm{O}\right) \\
\end{array}$ & $\begin{array}{c}\text { Soll } \\
\text { Moisture } \\
\text { Tonslon } \\
\left(\mathrm{cm} \mathrm{H}_{2} \mathrm{O}\right) \\
\end{array}$ & $\begin{array}{c}\text { Soll } \\
\text { Molsture } \\
\text { Tension } \\
\left(\mathrm{Cm} \mathrm{H}_{2} \mathrm{O}\right)\end{array}$ & 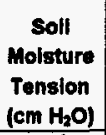 & $\begin{array}{c}\text { Soll } \\
\text { Molsture } \\
\text { Tension } \\
\left(\mathrm{Cm} \mathrm{H}_{2} \mathrm{O}\right) \\
\end{array}$ & 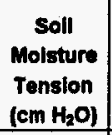 & $\begin{array}{c}\text { Soll } \\
\text { Moisture } \\
\text { Tension } \\
\left(\mathrm{Cm} \mathrm{H}_{2} \mathrm{O}\right)\end{array}$ & 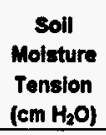 & $\begin{array}{c}\text { Soil } \\
\text { Moisture } \\
\text { Tonsion } \\
\left(\mathrm{Cm} \mathrm{H}_{2} \mathrm{O}\right)\end{array}$ & $\begin{array}{c}\text { Soll } \\
\text { Molsture } \\
\text { Tension } \\
\left(\mathrm{cm} \mathrm{H}_{2} \mathrm{O}\right) \\
\end{array}$ & $\begin{array}{c}\text { Soll } \\
\text { Moisture } \\
\text { Tension } \\
\left(\mathrm{Cm} \mathrm{H}_{2} \mathrm{O}\right) \\
\end{array}$ & $\begin{array}{c}\text { Soll } \\
\text { Molsture } \\
\text { Tension } \\
\left(\mathrm{Cm} \mathrm{H}_{2} \mathrm{O}\right)\end{array}$ & 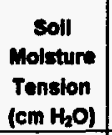 & $\begin{array}{c}\text { Soll } \\
\text { Moisture } \\
\text { Tension } \\
\left(\mathrm{em} \mathrm{H}_{2} \mathrm{O}\right) \\
\end{array}$ & $\begin{array}{c}\text { Soll } \\
\text { Moisture } \\
\text { Tension } \\
\text { (cm HzO) }\end{array}$ & $\begin{array}{c}\text { soll } \\
\text { Molsture } \\
\text { Tension } \\
\left(\mathrm{cm} \mathrm{H} \mathrm{H}_{2} \mathrm{O}\right)\end{array}$ & $\begin{array}{c}\text { Soll } \\
\text { Molature } \\
\text { Tenslon } \\
\text { (em } \mathrm{H}_{2} \mathrm{O} \text { ) }\end{array}$ \\
\hline Date & Time & Days' & C1" & $B 1^{\prime \prime}$ & $A 1^{\prime \prime}$ & $C 2^{b}$ & $B 2^{b}$ & $A 2^{b}$ & $\mathrm{C}^{\mathrm{C}}$ & $B 3^{c}$ & $A 3^{c}$ & $C 4^{d}$ & $B 4^{d}$ & $\mathrm{A4}^{\mathrm{d}}$ & $\mathrm{Cs}^{\circ}$ & $B 5^{\circ}$ & $A 5^{\circ}$ & $\mathrm{Cb}^{\prime}$ & $B 6^{\prime}$ & $A 6^{\prime}$ \\
\hline K\% & $0: 30$ & 2.004 & 102.8 & 98.0 & 99.1 & 87.3 & 89.8 & 89.2 & 82.3 & 73.8 & 82.2 & 52.1 & 48.1 & 58.1 & 83.7 & 71.7 & 65.2 & 100.1 & & \\
\hline $120 \%$ & $1: 00$ & 2.625 & 103.7 & 88.0 & 100.0 & 88.4 & 89.0 & 89.2 & 82.3 & 75.8 & 82.2 & 51.2 & 48.2 & 58.1 & 83.7 & 73.4 & 85.2 & 28.2 & & \\
\hline 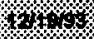 & \begin{tabular}{|l|}
$1: 30$ \\
\end{tabular} & 2.646 & 104.8 & 98.0 & 87.3 & 87.3 & 87.3 & 91.0 & 82.3 & 74.7 & 82.3 & 51.2 & 49.0 & 56.3 & 62.9 & 71.7 & 65.2 & 100.1 & & \\
\hline / 4 \% & $2: 00$ & 2.667 & 103.7 & 97.2 & 98.2 & 88.4 & 89.9 & 90.1 & 83.2 & 74.7 & 83.1 & 52.1 & 49.0 & 58.1 & 65.5 & 73.4 & 65.2 & 100.1 & & \\
\hline $124 \%$ & \begin{tabular}{|l|}
$2: 30$ \\
\end{tabular} & 2.688 & 105.5 & 98.9 & 99.1 & 87.3 & 89.0 & 89.2 & 82.3 & 77.3 & 83.1 & 49.5 & 49.0 & 58.1 & 83.8 & 72.6 & 66.1 & 100.2 & & \\
\hline $14 \%$ & $3: 00$ & 2.708 & 103.7 & 98.9 & 100.0 & 87.3 & 89.9 & 90.1 & 83.2 & 76.5 & 83.1 & 49.5 & 49.9 & 58.1 & 63.8 & 73.4 & 65.2 & 100.2 & & \\
\hline 124195 & 3:30 & 2.729 & 104.6 & 98.9 & 99.1 & 88.2 & 89.0 & 90.1 & 83.2 & 76.5 & 82.3 & 50.4 & 52.5 & 58.1 & 64.6 & 73.5 & 66.1 & 102.8 & & \\
\hline $4 \%$ & $4: 00$ & 2.750 & 105.5 & 98.0 & 99.1 & 88.2 & 90.8 & 90.1 & 84.0 & 77.4 & 84.0 & 50.4 & 49.8 & 58.1 & 94.7 & 73.5 & 66.1 & 101.1 & & \\
\hline 124018 & \begin{tabular}{|l|l}
$4: 30$ \\
\end{tabular} & 2.771 & 104.6 & 98.1 & 99.1 & 87.3 & 89.9 & 91.0 & 83.2 & 76.5 & 83.1 & 50.4 & 49.0 & 58.1 & 64.7 & 73.5 & 68.1 & 101.1 & & \\
\hline 101.48 & $5: 00$ & 2.792 & 106.4 & 98.9 & 99.1 & 88.2 & 90.8 & 80.1 & 83.2 & 76.5 & 83.1 & 51.2 & 49.9 & 58.1 & 68.4 & 73.5 & 86.1 & 101.4 & & \\
\hline 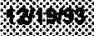 & $5: 00$ & 2.813 & 105.5 & 88.9 & 99.1 & 88.2 & 90.8 & 90.1 & 84.1 & 76.5 & 84,8 & 52.1 & 50.8 & 58.1 & 65.5 & 73.5 & 86.1 & 102.8 & & \\
\hline 12181 & $6: 00$ & 2.833 & 106.4 & 88.8 & 100.0 & 89.0 & 91.6 & 90.1 & 84.9 & 77.4 & 84.9 & 51.2 & 51.7 & 59.0 & 60.4 & 74.4 & 66.1 & 102.0 & & \\
\hline 1248 & \begin{tabular}{|l|l|}
$6: 30$ \\
\end{tabular} & 2.854 & 108.4 & 98.9 & 89.1 & 88.2 & 90.8 & 81.0 & 84.1 & 77.4 & 84.9 & 50.4 & 50.8 & 59.9 & 68.4 & 73.5 & 87.0 & 101.1 & & \\
\hline 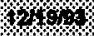 & $7: 00$ & 2.875 & 106.4 & 100.7 & 100.0 & 89.9 & 81.6 & 91.9 & 84.1 & 77.4 & 84.9 & 51.2 & 50.8 & 59.9 & 08.4 & 73.5 & 66.9 & 101.8 & & \\
\hline 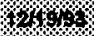 & $7: 30$ & 2.898 & 107.2 & 100.7 & 100.0 & 89.8 & 91.6 & 81.9 & 84.8 & $\pi 7.4$ & 84.9 & 51.2 & 52.5 & 60.7 & 87.3 & 73.5 & 88.9 & 101.1 & & \\
\hline$\% 4$ & $8: 00$ & 2.017 & 106.3 & 99.8 & 101.7 & 89.8 & 91.6 & 91.0 & 84.9 & 78.2 & 84.9 & 50.4 & 52.5 & 60.7 & 65.5 & 73.5 & 68.9 & 101.1 & & \\
\hline$x_{2}$ & $8: 30$ & 2.038 & 107.2 & 101.5 & 101.7 & 80.8 & 92.5 & 93.6 & 85.8 & 77.4 & 84.9 & 50.4 & 53.4 & 60.7 & 68.4 & 75.2 & 66,2 & 100.2 & & \\
\hline $17 \%$ & 9.00 & 2.058 & 107.2 & 101.5 & 102.6 & 89.9 & 22.5 & 92.7 & 86.6 & 78.2 & 84.9 & 49.5 & 53.4 & 60.7 & 65.5 & 75.2 & 68.6 & 100.2 & & \\
\hline $821 \%$ & $9: 30$ & 2.978 & 108.9 & 102.4 & 102.6 & 81.6 & 93.3 & 94.5 & 86.6 & 80.8 & 84.8 & 49.5 & 54.2 & 82.5 & 68.1 & 76.0 & 68.6 & 102.7 & & \\
\hline $3244 \%$ & $10: 00$ & 3.000 & 107.2 & 103.2 & 101.7 & 91.6 & 94.2 & 94.5 & 86.6 & 79.1 & 84.8 & 46.0 & 55.1 & 63.3 & 65.4 & 76.0 & 68.6 & 100.1 & & \\
\hline 17248 & $10: 30$ & 3.021 & 108.9 & 103.2 & 105.2 & 82.4 & 93.3 & 85.3 & 87.4 & 81.7 & 84.8 & 45.8 & 55.0 & 83.3 & 68.3 & 77.7 & 69.4 & 97.4 & & \\
\hline $8 \%$ & 11:00 & 3.042 & 110.6 & 104.0 & 106.9 & 83.3 & 95.8 & 97.0 & 88.2 & 82.6 & 85.7 & 45.9 & 56.7 & 85.0 & 67.1 & 76.8 & 70.2 & 97.3 & & \\
\hline $1 / 2 \% \%$ & $11: 30$ & 3.063 & 110.5 & 104.8 & 106.8 & 84.1 & 96.6 & 97.0 & 89.1 & 82.6 & 86.5 & 46.8 & 57.6 & 85.0 & 67.0 & 79.3 & 71.0 & 87.3 & & \\
\hline / 18 & \begin{tabular}{|l|}
$12: 00$ \\
\end{tabular} & 3.083 & 110.5 & 105.7 & 108.5 & 94.9 & 97.5 & 97.0 & 90.8 & 84.3 & 88.2 & 46.7 & 57.5 & 85.0 & 67.0 & 79.3 & 71.9 & 08.1 & & \\
\hline $4 \%$ & $12: 30$ & 3.104 & 112.2 & 105.7 & 106.8 & 84.9 & 97.5 & 98.7 & 89.8 & 83.4 & 86.5 & 48.7 & 57.5 & 85.8 & 86.9 & 80.1 & 71.0 & 97.2 & & \\
\hline (4, & $13: 00$ & 3.125 & 112.2 & 106.5 & 108.5 & 95.8 & 97.4 & 98.7 & 90.7 & 86.0 & 89.9 & 53.7 & 56.6 & 65.0 & 69.5 & 80.1 & 72.6 & 105.9 & & \\
\hline 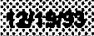 & 13:30 & 3.146 & 112.2 & 107.4 & 108.5 & 84.8 & 97.4 & 98.7 & 89.8 & 84.3 & 87.3 & 49.3 & 58.3 & 65.8 & 68.9 & 79.2 & 71.8 & 100.6 & & \\
\hline (2) & $14: 00$ & 3.167 & 112.2 & 108.5 & 108.5 & 95.7 & 99.1 & 98.7 & 90.6 & 88.7 & 89.9 & 53.7 & 58.3 & 66.7 & 68.4 & 80.0 & 73.4 & 109.3 & & \\
\hline 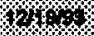 & $14: 30$ & 3.188 & 112.2 & 108.5 & 108.4 & 96.6 & 99.1 & 89.5 & 02.4 & 88.6 & 89.8 & 58.0 & 59.1 & 68.7 & 720 & 80.8 & 73.4 & 111.0 & & \\
\hline$\%$ & $15: 00$ & 3.208 & 112.2 & 106.5 & 107.6 & 85.7 & 97.4 & 98.7 & 91.5 & 86.0 & 88.2 & 56.3 & 58.3 & 65.8 & 68.6 & 80.8 & 72.6 & 109.3 & & \\
\hline 2 & $15: 30$ & 3.229 & 112.2 & 106.5 & 108.4 & 95.7 & 97.4 & 99.5 & 91.5 & 87.8 & 88.1 & 53.7 & 59.1 & 65.8 & 69.4 & 81.7 & 71.7 & 105.7 & & \\
\hline 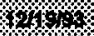 & $16: 00$ & 3.260 & 113.0 & 108.4 & 108.4 & 85.7 & 99.1. & 99.5 & 81.5 & 88.6 & 80.7 & 50.3 & 59.1 & 65.8 & 71.1 & 81.7 & 73.4 & 108.6 & & \\
\hline 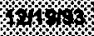 & $16: 30$ & 3.271 & 111.3 & 106.4 & 107.6 & 85.7 & 99.1 & 98.7 & 01.5 & 88.6 & 80.7 & 58.8 & 59.1 & 66.6 & 71.1 & 80.8 & 72.5 & 109.2 & & \\
\hline$\%$ & $17: 00$ & 3.292 & 112.2 & 106.5 & 108.4 & 26.6 & 99.1 & 98.7 & 91.5 & 88.6 & 80.7 & 59.8 & 80.0 & 66.7 & 72.0 & 81.7 & 73.4 & 100.2 & & \\
\hline $10 \%$ & $17: 30$ & 3.313 & 112.2 & 104.7 & 107.8 & 94.8 & 97.4 & 88.7 & 81.5 & 86.9 & 80.8 & 58.9 & 58.3 & 66.7 & 72.0 & 80.8 & 73.4 & 100.3 & & \\
\hline $4 \%$ & $18: 00$ & 3.333 & 110.5 & 104.8 & 108.7 & 84.8 & 99.1 & 98.7 & 80.7 & 86.9 & 89.8 & 58.0 & 80.0 & 86.7 & 71.2 & 80.8 & 73.5 & 108.5 & & \\
\hline 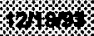 & $18: 30$ & 3.364 & 110.5 & 105.7 & 100.8 & 94.0 & 98.3 & 97.0 & 80.7 & 86.0 & 89.8 & 57.2 & 59.2 & 66.7 & 71.2 & 81.0 & 72.7 & 107.6 & & \\
\hline 1624. & $19: 00$ & 3.375 & 110.5 & 104.8 & 108.8 & 94.8 & 98.3 & 97.0 & 81.6 & 86.0 & 89.8 & 50.3 & 58.4 & 67.6 & 71.3 & 81.0 & 72.7 & 107.7 & & \\
\hline
\end{tabular}


Table 5. Tensiometer Data

\begin{tabular}{|c|c|c|c|c|c|c|c|c|c|c|c|c|c|c|c|c|c|c|c|c|}
\hline & & & & & & & & & Ten & siometer & Data & & & & & & & & & \\
\hline & & Test & $\begin{array}{c}\text { Soil } \\
\text { Moisture } \\
\text { Tension } \\
\left(\mathrm{em} \mathrm{H}_{2} \mathrm{O}\right) \\
\end{array}$ & 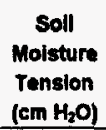 & $\begin{array}{c}\text { Soll } \\
\text { Moisture } \\
\text { Tension } \\
\left(\mathrm{Cm} \mathrm{H}_{2} \mathrm{O}\right) \\
\end{array}$ & $\begin{array}{c}\text { Soll } \\
\text { Molsture } \\
\text { Tension } \\
\left(\mathrm{Cm} \mathrm{H}_{2} \mathrm{O}\right) \\
\end{array}$ & $\begin{array}{c}\text { Soll } \\
\text { Molsture } \\
\text { Tension } \\
\left(\mathrm{Cm} \mathrm{H}_{2} \mathrm{O}\right) \\
\end{array}$ & $\begin{array}{c}\text { Soll } \\
\text { Molsture } \\
\text { Tension } \\
\text { (cm } \mathrm{H}_{2} \text { O) }\end{array}$ & $\begin{array}{c}\text { Soll } \\
\text { Moisture } \\
\text { Tenslon } \\
\left(\mathrm{em} \mathrm{H}_{2} \mathrm{O}\right) \\
\end{array}$ & $\begin{array}{c}\text { Soll } \\
\text { Molsture } \\
\text { Tension } \\
\left(\mathrm{Cm} \mathrm{H}_{2} \mathrm{O}\right) \\
\end{array}$ & $\begin{array}{c}\text { Soll } \\
\text { Molsture } \\
\text { Tension } \\
\left(\mathrm{cm} \mathrm{H}_{2} \mathrm{O}\right)\end{array}$ & $\begin{array}{c}\text { Solf } \\
\text { Molsture } \\
\text { Tension } \\
\left(\mathrm{cm} \mathrm{H}_{2} \mathrm{O}\right) \\
\end{array}$ & $\begin{array}{c}\text { Soll } \\
\text { Molsture } \\
\text { Tension } \\
\left(\mathrm{cm} \mathrm{H}_{2} \mathrm{O}\right)\end{array}$ & $\begin{array}{c}\text { Soll } \\
\text { Molsture } \\
\text { Tension } \\
\text { (em } \mathrm{H}_{2} \mathrm{O} \text { ) } \\
\end{array}$ & $\begin{array}{c}\text { Soll } \\
\text { Moisture } \\
\text { Tension } \\
\left(\mathrm{cm} \mathrm{H}_{2} \mathrm{O}\right)\end{array}$ & $\begin{array}{c}\text { Soil } \\
\text { Molstume } \\
\text { Tension } \\
\left(\mathrm{C} \mathrm{H}_{2} \mathrm{O}\right) \\
\end{array}$ & $\begin{array}{c}\text { Solf } \\
\text { Molsture } \\
\text { Tension } \\
\text { (cm } \mathrm{H}_{2} \mathrm{O} \text { 이 }\end{array}$ & $\begin{array}{c}\text { Soll } \\
\text { Moisture } \\
\text { Tension } \\
\left(\mathrm{Cm} \mathrm{H}_{2} \mathrm{O}\right)\end{array}$ & $\begin{array}{c}\text { Soll } \\
\text { molature } \\
\text { Tension } \\
\left(\mathrm{Cm} \mathrm{H}_{2} \mathrm{O}\right)\end{array}$ & $\begin{array}{c}\text { Soll } \\
\text { Molsture } \\
\text { Tension } \\
\left(\mathrm{Cm} \mathrm{H}_{2} \mathrm{O}\right) \\
\end{array}$ \\
\hline Date & Time & Days' & $\mathrm{C1}^{\circ}$ & $B 1^{\prime \prime}$ & $A 1^{*}$ & $\mathrm{Cl}^{\mathrm{b}}$ & $B 2^{b}$ & $A 2^{\circ}$ & $\mathrm{C3}^{\mathrm{C}}$ & $\mathrm{B3}^{\mathrm{C}}$ & $\mathrm{A}^{\mathrm{C}}$ & $C 4^{d}$ & $B 4^{d}$ & $\mathrm{A4}^{\mathrm{d}}$ & $C 5^{\circ}$ & $\mathrm{B6}^{\circ}$ & $A 5^{\circ}$ & $\mathrm{Cb}^{\prime}$ & $86^{\prime}$ & $A 6^{\prime}$ \\
\hline $8 \% \%$ & \begin{tabular}{|l|}
$19: 30$ \\
\end{tabular} & 3.396 & 111.4 & 105.7 & 106.8 & 95.8 & 98.3 & 98.7 & 91.6 & 86.1 & 89.9 & 57.2 & 59.2 & 66.7 & 71.3 & 80.2 & 71.8 & 108.6 & & \\
\hline 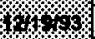 & $20: 00$ & 3.417 & 111.4 & 104.8 & 106.8 & 94.9 & 88.3 & 97.8 & 81.6 & 86.9 & 80.0 & 57.2 & 59.3 & 66.7 & 72.2 & 81.9 & 73.6 & 108.8 & & \\
\hline $12 \% \%$ & $20: 30$ & 3.438 & 110.5 & 104.8 & 106.8 & 95.0 & 97.5 & 97.8 & 91.7 & 88.1 & 90.0 & 56.4 & 59.3 & 66.7 & 72.2 & 80.2 & 68.4 & 107.7 & & \\
\hline (12) & $21: \infty$ & 3.458 & 111.4 & 104.8 & 106.0 & 95.0 & 97.5 & 98.7 & 91.7 & 86.1 & 90.8 & 56.4 & 57.6 & 66.7 & 73.1 & 81.1 & 71.8 & 107.8 & & \\
\hline $11401 \%$ & $21: 30$ & 3.479 & 111.4 & 104.8 & 106.8 & 95.0 & 98.4 & 98.7 & 92.5 & 87.0 & 91.7 & 56.4 & 59.3 & 87.6 & 72.2 & 82.0 & 73.6 & 108.7 & & \\
\hline $17 \%$ & $22: 00$ & 3.500 & 112.3 & 105.7 & 106.0 & 95.9 & 97.5 & 98.8 & 81.7 & 87.0 & 91.7 & 56.4 & 58.4 & 67.6 & 72.2 & 80.2 & 73.7 & 108.7 & & \\
\hline $87 \%$ & $22: 30$ & 3.521 & 112.3 & 105.7 & 106.9 & 95.9 & 98.4 & 97.9 & 92.6 & 86.1 & 91.7 & 56.4 & 59.3 & 68.5 & 73.1 & 82.0 & 73.7 & 108.7 & & \\
\hline 21/ & $23: 00$ & 3.542 & 111.4 & 104.8 & 106.8 & 95.9 & 99.3 & 98.8 & 92.8 & 86.1 & 91.7 & 56.4 & 59.3 & 67.6 & 73.1 & 82.0 & 74.5 & 108.7 & & \\
\hline $891 \%$ & $23: 30$ & 3.563 & 111.5 & 105.8 & 106.8 & 95.9 & 97.6 & 97.8 & 91.7 & 87.0 & 91.7 & 56.4 & 59.3 & 68.5 & 74.0 & 81.1 & 75.4 & 109.6 & & \\
\hline $8020 \%$ & $0: 00$ & 3.583 & 111.5 & 105.8 & 106.9 & 95.9 & 98.4 & 98.8 & 92.6 & 85.2 & 81.7 & 56.4 & 59.4 & 69.4 & 74.9 & 81.2 & 75.4 & 109.6 & & \\
\hline 1270.6 & $0: 30$ & 3.604 & 112.4 & 104.9 & 106.0 & 95.0 & 99.3 & 98.8 & 94.3 & 87.9 & 91.8 & 58.4 & 59.4 & 69.4 & 75.8 & 82.1 & 75.4 & 108.8 & & \\
\hline $124 \%$ & $1: 00$ & 3.625 & 112.4 & 105.8 & 108.0 & 95.9 & 98.4 & 97.8 & 93.5 & 87.8 & 91.8 & 56.4 & 59.4 & 69.4 & 75.8 & 82.1 & 75.5 & 110.5 & & \\
\hline $42 \% 0 \%$ & $1: 30$ & 3.646 & 113.2 & 104.9 & 106.9 & 96.8 & 99.3 & 98.8 & 93.5 & 87.9 & 90.9 & 56.4 & 59.4 & 69.4 & 76.7 & 82.1 & 75.5 & 109.7 & & \\
\hline $151 . \%$ & $2: 00$ & 3.667 & 112.4 & 104.8 & 105.2 & 96.8 & 99.3 & 98.8 & 91.8 & 87.0 & 91.8 & 56.4 & 60.3 & 69.5 & 74.1 & 81.2 & 76.4 & 110.8 & & \\
\hline 121045 & $2: 30$ & 3.688 & 112.4 & 105.0 & 108.1 & 95.9 & 97.6 & 98.8 & 93.5 & 87.0 & 91.8 & 56.4 & 60.3 & 69.5 & 73.2 & 82.1 & 75.5 & 110.8 & & \\
\hline 12. & $3: 00$ & 3.708 & 112.4 & 108.7 & 106.1 & 87.7 & 98.5 & 98.8 & 93.5 & 87.9 & 91.8 & 56.4 & 58.6 & 69.5 & 72.4 & 82.1 & 75.5 & 109.7 & & \\
\hline $1 \% 1 \%$ & 3:30 & 3.729 & 112.4 & 105.8 & 108.8 & 96.8 & 99.4 & 98.8 & 93.5 & 87.9 & 91.8 & 56.4 & 60.3 & 69.5 & 70.7 & 82.1 & 75.5 & 111.5 & & \\
\hline $180 \% 5$ & $4: 00$ & 3.760 & 114.1 & 105.8 & 106.8 & 87.7 & 99.4 & 99.7 & 93.5 & 87.9 & 91.8 & 56.4 & 80.3 & 69.5 & 70.7 & 82.1 & 76.4 & 1111.5 & & \\
\hline $10.4 \%$ & $4: 30$ & 3.771 & 113.3 & 106.7 & 106.9 & 97.7 & 101.1 & 98.8 & 93.5 & 87.9 & 93.5 & 56.4 & 60.3 & 69.5 & 70.7 & 82.1 & 78.4 & 111.5 & & \\
\hline 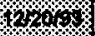 & $5: 00$ & 3.792 & 114.1 & 105.8 & 108.9 & 96.8 & 99.4 & 99.7 & 83.5 & 88.8 & 82.7 & 54.7 & 60.3 & 69.5 & 70.7 & 82.1 & 75.5 & 110.6 & & \\
\hline $824 \% 1$ & $5: 30$ & 3.813 & 113.3 & 106.7 & 108.1 & 96.0 & 99.4 & 98.8 & 83.5 & 87.9 & 93.5 & 58.5 & 60.3 & 71.2 & 71.5 & 83.8 & 76.4 & 111.5 & & \\
\hline 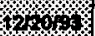 & $6: 00$ & 3.833 & 114.2 & 106.7 & 107.8 & 97.7 & 98.5 & 99.7 & 93.6 & 87.9 & 92.7 & 56.5 & 62.1 & 71.2 & 71.5 & 83.0 & 77.3 & 110.8 & & \\
\hline $12104 \%$ & $6: 30$ & 3.854 & 114.2 & 108.7 & 107.0 & 96.8 & 99.4 & 99.7 & 93.6 & 87.9 & 93.5 & 56.5 & 62.1 & 71.2 & 70.7 & 83.0 & 77.3 & 112.4 & & \\
\hline 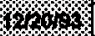 & \begin{tabular}{|l|}
$7: 00$ \\
\end{tabular} & 3.876 & 115.0 & 106.7 & 106.1 & 96.8 & 101.1 & 100.6 & 83.6 & 87.9 & 93.5 & 56.5 & 61.2 & 71.2 & 70.7 & 83.9 & 77.3 & 111.5 & & \\
\hline 4 . & $7: 30$ & 3.896 & 114.2 & 106.7 & 107.0 & 98.8 & 100.2 & 98.8 & 93.6 & 88.8 & 93.5 & 55.6 & 61.2 & 71.2 & 70.7 & 83.0 & 77.3 & 112.4 & & \\
\hline 10209 & $8: 00$ & 3.917 & 114.2 & 106.7 & 107.0 & 96.8 & 101.1 & 102.3 & 93.6 & 87.1 & 827 & 56.5 & 62.1 & 71.2 & 70.7 & 83.0 & 76.4 & 112.4 & & \\
\hline $192 \%$. & $8: 30$ & 3.938 & 115.0 & 106.7 & 107.8 & 97.7 & 100.2 & 101.5 & 93.5 & 87.9 & 91.8 & 53.8 & 62.0 & 70.4 & 69.8 & 83.0 & 77.3 & 110.8 & & \\
\hline \%1\% & $9: 00$ & 3.958 & 115.0 & 108.7 & 108.7 & 98.6 & 101.1 & 101.4 & 93.5 & 88.8 & 91.8 & 53.0 & 62.0 & 72.1 & 72.4 & 83.9 & 77.2 & 110.6 & & \\
\hline $120 \times 4$ & $8: 30$ & 3.979 & 114.1 & 106.7 & 108.7 & 98.5 & 101.1 & 101.4 & 95.3 & 88.8 & 91.8 & 52.9 & 62.0 & 73.0 & 71.5 & 84.7 & 79.0 & 111.5 & & \\
\hline $85004 \%$ & $10: 00$ & 4.000 & 115.8 & 109.3 & 108.7 & 99.4 & 101.1 & 102.3 & 95.2 & 88.7 & 91.8 & 49.4 & 63.7 & 73.0 & 74.8 & 86.5 & 78.8 & 100.7 & & \\
\hline $82 \% 0$ & $10: 30$ & 4.021 & 115.8 & 109.3 & 109.5 & 99.4 & 102.8 & 103.2 & 97.0 & 90.6 & 93.5 & 49.4 & 65.5 & 73.0 & 74.0 & 85.6 & 78.9 & 108.8 & & \\
\hline 42,250 & $11: 00$ & 4.042 & 115.8 & 109.2 & 112.1 & 102.0 & 103.6 & 103.2 & 96.8 & 91.4 & 94.4 & 48.5 & 65.4 & 73.8 & 75.8 & 85.5 & 79.8 & 109.6 & & \\
\hline 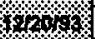 & 11:30 & 4.063 & 116.7 & 110.1 & 113.0 & 102.0 & 104.5 & 104.0 & 96.9 & 90.5 & 85.2 & 47.7 & 65.4 & 72.9 & 75.7 & 86.4 & 80.6 & 107.8 & & \\
\hline $4 \%$ & \begin{tabular}{|l|}
$12: 00$ \\
\end{tabular} & 4.083 & 117.5 & 111.0 & 113.0 & 102.0 & 104.5 & 104.0 & 96.9 & 90.5 & 85.2 & 48.5 & 67.2 & 74.7 & 75.7 & 87.3 & 80.6 & 108.7 & & \\
\hline 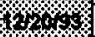 & \begin{tabular}{|l|}
$12: 30$ \\
\end{tabular} & 4.104 & 117.5 & 110.8 & 113.8 & 102.8 & 104.4 & 100.6 & 97.8 & 93.2 & 88.1 & 48.5 & 87.1 & 74.7 & 77.4 & 87.2 & 80.6 & 108.7 & & \\
\hline $12 \% 2 \%$ & \begin{tabular}{|l|}
$13: 00$ \\
\end{tabular} & 4.126 & 117.5 & 140.8 & 114.7 & 101.9 & 105.3 & 107.5 & 97.7 & 93.2 & 96.1 & 50.3 & 68.0 & 75.5 & 78.3 & 87.2 & 81.4 & 110.4 & & \\
\hline (27, & $13: 30$ & 4.146 & 118.3 & 111.8 & 114.7 & 102.8 & 107.0 & 107.5 & 98.6 & 94.0 & 96.0 & 52.0 & 67.9 & 75.5 & 79.1 & 87.1 & 81.3 & 110.3 & & \\
\hline 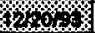 & $14: 00$ & 4.107 & 118.3 & 111.7 & 114.6 & 103.6 & 108.1 & 108.6 & 98.5 & 94.0 & 96.0 & 54,6 & 67.0 & 75.5 & 80.7 & 88.0 & 80.4 & $\$ 11.1$ & & \\
\hline
\end{tabular}


Table 5. Tensiometer Data

\begin{tabular}{|c|c|c|c|c|c|c|c|c|c|c|c|c|c|c|c|c|c|c|c|c|}
\hline & & & & & & & & & Ten: & siometer & Data & & & & & & & & & \\
\hline & & Test & $\begin{array}{c}\text { Soll } \\
\text { Molsture } \\
\text { Tension } \\
\left.\text { (em } \mathrm{H}_{2} \mathrm{O}\right)\end{array}$ & $\begin{array}{c}\text { Soll } \\
\text { Moisture } \\
\text { Tenston } \\
\left(\mathrm{cm} \mathrm{H} \mathrm{H}_{2} \mathrm{O}\right)\end{array}$ & $\begin{array}{c}\text { Soll } \\
\text { Moisture } \\
\text { Tension } \\
\left(\mathrm{Cm} \mathrm{H}_{2} \mathrm{O}\right) \\
\end{array}$ & $\begin{array}{c}\text { Soll } \\
\text { Molsture } \\
\text { Tension } \\
\left(\mathrm{Cm} \mathrm{H}_{2} \mathrm{O}\right) \\
\end{array}$ & $\begin{array}{c}\text { Soll } \\
\text { Moisture } \\
\text { Tension } \\
\left(\mathrm{Cm}_{2} \mathrm{O}\right) \\
\end{array}$ & $\begin{array}{c}\text { Soll } \\
\text { Moisture } \\
\text { Tension } \\
\left(\mathrm{Cm} \mathrm{H}_{2} \mathrm{O}\right) \\
\end{array}$ & $\begin{array}{c}\text { Soll } \\
\text { Molsture } \\
\text { Tension } \\
\left.\text { (cm } \mathrm{H}_{2} \mathrm{O}\right) \\
\end{array}$ & $\begin{array}{c}\text { Soll } \\
\text { Moisture } \\
\text { Tenslon } \\
\left(\mathrm{cm}_{2} \mathrm{O}\right) \\
\end{array}$ & $\begin{array}{c}\text { Soll } \\
\text { Moisture } \\
\text { Tension } \\
\left(\mathrm{Cm} \mathrm{H}_{2} \mathrm{O}\right) \\
\end{array}$ & 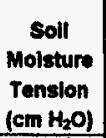 & $\begin{array}{c}\text { Soll } \\
\text { Moisture } \\
\text { Tension } \\
\left(\mathrm{cm} \mathrm{H}_{2} \mathrm{O}\right)\end{array}$ & $\begin{array}{c}\text { Soll } \\
\text { Molature } \\
\text { Tension } \\
\left(\mathrm{Cm} \mathrm{H}_{2} \mathrm{O}\right)\end{array}$ & $\begin{array}{c}\text { Soll } \\
\text { Molsture } \\
\text { Tension } \\
\left(\mathrm{Cm} \mathrm{H}_{2} \mathrm{O}\right)\end{array}$ & $\begin{array}{c}\text { Soll } \\
\text { Moisture } \\
\text { Tonsion } \\
\left(\mathrm{em} \mathrm{H}_{2} \mathrm{O}\right) \\
\end{array}$ & $\begin{array}{c}\text { Soll } \\
\text { Molsture } \\
\text { Tenalon } \\
\left(\mathrm{cm} \mathrm{H}_{2} \mathrm{O}\right) \\
\end{array}$ & $\begin{array}{c}\text { soll } \\
\text { Molisture } \\
\text { Tension } \\
\left(\mathrm{Cm} \mathrm{H}_{2} \mathrm{O}\right) \\
\end{array}$ & $\begin{array}{c}\text { Soll } \\
\text { Molsture } \\
\text { Tenstion } \\
\left(\mathrm{Cm} \mathrm{H}_{2} \mathrm{O}\right) \\
\end{array}$ & 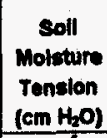 \\
\hline Date & Time & Days' & $\mathrm{C1}^{\prime \prime}$ & $B 1^{\circ}$ & $A 1^{n}$ & $\mathrm{C2}^{\mathrm{b}}$ & $B 2^{6}$ & $A Z^{b}$ & $\mathrm{C3}^{\mathrm{C}}$ & $\mathrm{B3}^{\mathrm{c}}$ & $\mathrm{AB}^{\mathrm{C}}$ & $\mathrm{C}^{\mathrm{d}}$ & $\mathrm{BA}^{\mathrm{d}}$ & $\mathrm{A4}^{\mathrm{d}}$ & $C 5^{\circ}$ & $B 5^{\circ}$ & $A 5^{\circ}$ & $\mathrm{co}^{\prime}$ & $86^{6}$ & $A 6^{\prime}$ \\
\hline KF\% & $14: 30$ & 4.188 & 118.1 & 111.7 & $\$ 13.7$ & 103.5 & 105.2 & 107.4 & 99.3 & 94.0 & 97.7 & 56.3 & 67.8 & 74.6 & 81.6 & 87.8 & 82.1 & 113.7 & & \\
\hline 4,2018 & $15: 00$ & 1.206 & 118.2 & 111.7 & 114.6 & 103.5 & 106.0 & 105.7 & 89.3 & 94.8 & 97.7 & 57.2 & 68.8 & 78.3 & 81.5 & 87.8 & 82.1 & 114.5 & & \\
\hline Y r & $16: 00$ & 4.250 & 118.2 & 112.5 & 114.5 & 102.6 & 105.1 & 105.6 & 101.0 & 94.8 & 98.5 & 59.7 & 68.9 & 75.4 & 84.9 & 87.8 & 82.0 & 117.0 & & \\
\hline 82,01 & $18: 00$ & 4.333 & 117.5 & 110.0 & 112.1 & 104.9 & 104.4 & 104.0 & 88.6 & 93.1 & 98.6 & 60.7 & 85.4 & 74.6 & 85.2 & 87.2 & 81.4 & 118.2 & & \\
\hline 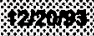 & $20: 00$ & 4.417 & 117.6 & 110.2 & 110.4 & 102.1 & 103.7 & 103.2 & 88.8 & 91.5 & 98.1 & 58.2 & 65.5 & 74.7 & 83.6 & 85.6 & 80.7 & 115.0 & & \\
\hline $1700 \%$ & $22: 00$ & 4.500 & 117.7 & 110.2 & 109.8 & 101.2 & 104.6 & 104.1 & 97.9 & 91.5 & 97.0 & 57.4 & 65.6 & 75.7 & 84.6 & 85.7 & 80.8 & 113.3 & & \\
\hline 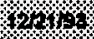 & 0.00 & 4.583 & 197.7 & 110.2 & 109.8 & 102.1 & 104.6 & 104.1 & 97.1 & 80.7 & 97.1 & 57.4 & 65.6 & 75.7 & 74.2 & 87.5 & 80.8 & 916.0 & & \\
\hline 121.14 & $2: 00$ & 4.667 & 117.7 & 110.3 & 109.7 & 102.2 & 104.7 & 104.1 & 98.9 & 90.7 & 98.0 & 57.4 & 65.7 & 75.7 & 73.4 & 87.5 & 80.9 & 118.7 & & \\
\hline 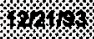 & 4:00 & 4.750 & 117.7 & 111.1 & 108.8 & 103.0 & 105.5 & 104.1 & 88.0 & 90.7 & 98.0 & 54.8 & 65.7 & 75.7 & 70.8 & 86.7 & 81.8 & 917.8 & & \\
\hline 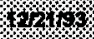 & $6: 00$ & 4.833 & 118.6 & 110.3 & 111.4 & 103.9 & 102.8 & 104.1 & 88.8 & 80.7 & 98.0 & 53.8 & 65.7 & 75.7 & 70.8 & 86.7 & 80.9 & 117.0 & & \\
\hline 148,15 & $8: 00$ & 4.017 & 120.3 & 912.0 & 193.1 & 104.8 & 105.5 & 406.8 & 100.6 & 82.4 & 98.8 & 52.2 & 67.4 & 77.5 & 76.9 & 88.4 & 80.8 & 116.1 & & \\
\hline $12 \% 4 \%$ & $10: 00$ & 5.000 & 120.3 & 109.4 & 114.8 & 104.7 & 108.1 & 105.8 & 100.6 & 92.4 & 98.8 & 84.4 & 68.5 & 74.8 & 84.6 & 87.5 & 79.1 & 110.7 & & \\
\hline $10 \% 16$ & $12: 00$ & 6.083 & 120.2 & 113.7 & 114.8 & 105.5 & 109.7 & 107.6 & 98.8 & 91.5 & 98.8 & 73.1 & 89.0 & 79.2 & 80.6 & 88.3 & 83.3 & 108.9 & & \\
\hline 52.4 & $14: 00$ & 6.167 & 122.8 & 117.1 & 117.4 & 108.1 & 109.6 & 111.0 & 103.0 & 85.9 & 100.5 & 78.3 & 72.4 & 80.8 & 93.9 & 80.8 & 85.8 & 114.0 & & \\
\hline 18,142 & $16: 00$ & 5.250 & 123.6 & 118.2 & 116.5 & 108.1 & 109.7 & 110.2 & 104.7 & 96.7 & 103.1 & 81.8 & 73.3 & 81.7 & 85.8 & 80.8 & 88.7 & 118.4 & & \\
\hline 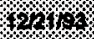 & $18: 00$ & 5.333 & 122.8 & 113.6 & 115.7 & 107.2 & 100.7 & 408.4 & 103.8 & 85.9 & 102.2 & 84.4 & 72.4 & 80.9 & 96.6 & 80.8 & 85.9 & 120.2 & & \\
\hline 1 $12 x$ & $20: 00$ & 8.417 & 122.9 & 113.7 & 115.7 & 109.0 & 108.8 & 108.5 & 104.0 & 95.9 & 102.3 & 84.4 & 725 & 80.0 & 86.8 & 80.9 & 85.9 & 117.7 & & \\
\hline $1 / 2110$ & $22: 00$ & 6.600 & 122.9 & 115.4 & 115.7 & 109.0 & 109.7 & 109.3 & 104.0 & 95.9 & 102.3 & 85.3 & 72.5 & 80.8 & 97.5 & 91.8 & 88.8 & 118.6 & & \\
\hline $14322 \%$ & $0: 00$ & 5.683 & 122.9 & 114.5 & 114.8 & 109.0 & 100.8 & 108.5 & 104.8 & 95.0 & 102.3 & 87.1 & 74.2 & 81.8 & 87.5 & 91.8 & 87.7 & 118.6 & & \\
\hline 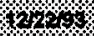 & $2: 00$ & 6.667 & 123.8 & 114.8 & 115.7 & 109.1 & 110.6 & 108.5 & 104.8 & 95.9 & 103.1 & 88.0 & 73.4 & 82.7 & 98.4 & 91.8 & 87.7 & 120.4 & & \\
\hline $12 \%$ & $4: 00$ & 5.750 & 124.7 & 118.3 & 115.7 & 109.1 & 110.7 & 109.4 & 106.6 & 96.8 & 103.2 & 88.0 & 74.3 & 82.7 & 98.4 & 80.1 & 88.6 & & & \\
\hline $892 \%$ & $6: 00$ & 5.833 & 124.7 & 115.5 & 114.0 & 109.1 & 111.5 & 109.4 & 105.8 & 96.9 & 104.0 & 88.0 & 75.2 & 82.7 & 99.3 & 92.8 & 89.5 & & & \\
\hline 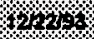 & $8: 00$ & 5.017 & 124.7 & 916.3 & 116.8 & 110.0 & 111.5 & 111.1 & 107.5 & 98.6 & 104.9 & 88.8 & 78.0 & 82.7 & 101.0 & 92.7 & 89.5 & & & \\
\hline 812140 & $10: 00$ & 6.000 & 128.4 & 118.9 & 121.0 & 111.7 & 115.0 & 112.9 & 107.5 & 99.5 & 105.8 & 87.1 & 77.8 & 86.2 & 99.3 & 96.2 & 90.3 & & & \\
\hline 412218 & $12: 00$ & 0.083 & 128.1 & 120.6 & 121.8 & 113.3 & 114.9 & 114.6 & 108.3 & 101.2 & 108.6 & 85.3 & 79.4 & 87.0 & 100.8 & 97.0 & 92.8 & & & \\
\hline $1.72,1$. & $14: 00$ & 0.167 & 128.7 & 122.2 & 123.5 & 115.0 & 116.5 & 116.3 & 111.8 & 104.7 & 107.4 & 88.8 & 80.2 & 88.7 & 101.7 & 97.7 & 93.6 & & & \\
\hline $80 z \%$ & $18: 00$ & 6.250 & 127.9 & 122.2 & 123.4 & 114.9 & 116.4 & 116.2 & 111.5 & 103.7 & 107.3 & 80.4 & 80.8 & 88.7 & 104.1 & 97.6 & 24.3 & & & \\
\hline 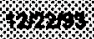 & $18: 00$ & 6.333 & 128.0 & 118.8 & 118.1 & 113.3 & 114.8 & 113.8 & 110.8 & 101.2 & 107.4 & 83.1 & 79.4 & 87.0 & 103.4 & 95.2 & 92.7 & & & \\
\hline 112101 & $20: 00$ & 6.417 & 128.1 & 118.0 & 119.2 & 113.4 & 115.0 & 112.8 & 109.2 & 99.5 & 108.6 & 93.2 & 78.6 & 86.2 & 101.8 & 94.4 & 91.2 & & & \\
\hline$y_{1}$ & $22: 00$ & 6.600 & 128.2 & 118.1 & 118.4 & 113.5 & 114.2 & 113.8 & 109.3 & 99.5 & 106.7 & 92.4 & 78.7 & 86.2 & 102.0 & 94.5 & 91.3 & & & \\
\hline 82,50 & $0: 00$ & 6.683 & 128.2 & 118.1 & 118.7 & 114.4 & 115.0 & 113.8 & 109.3 & 99.5 & 108.7 & 93.3 & 77.8 & 88.3 & 102.0 & 94.6 & 80.4 & & & \\
\hline 821. & $2: 00$ & 0.667 & 126.5 & 119.0 & 118.4 & 113.5 & & 113.8 & 110.2 & 100.4 & 106.7 & 83.7 & 77.0 & 85.4 & 102.0 & 94.6 & 81.3 & & & \\
\hline 3. & $4: 00$ & 6.750 & 128.2 & 119.0 & 117.6 & 113.5 & & 112.9 & 191.1 & 100.5 & 107.8 & 82.8 & 72.7 & 86.3 & 102.9 & 95.5 & 92.2 & & & \\
\hline $12=1 \%$ & $6: 00$ & 6.833 & 129.1 & 119.0 & 118.5 & 912.7 & & 114.7 & 110.2 & 88.7 & 107.6 & 88.1 & 77.0 & 87.2 & 102.9 & 86.4 & 91.4 & & & \\
\hline 4 & $8: 00$ & 6.817 & 128.1 & 119.8 & 120.2 & 115.3 & & 114.7 & $\mathbf{1 1 1 . 1}$ & 101.3 & 107.6 & 78.5 & 75.3 & 87.2 & 102.9 & 97.2 & 92.2 & & & \\
\hline 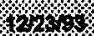 & $10: 00$ & 7.000 & 129.0 & 122.4 & 121.0 & 116.1 & & 115.5 & 111.0 & 95.1 & 105.8 & 98.5 & 77.0 & 88.8 & 102.0 & 68.3 & 80.4 & & & \\
\hline $82,1 \%$ & $12: 00$ & 7.083 & 133.3 & 125.8 & 126.2 & 120.3 & 118.4 & 118.8 & 118.1 & 108.3 & 112.7 & 81.5 & 84.6 & 81.4 & 108.1 & 101.4 & 88.9 & & & \\
\hline$\gamma_{2} \gamma_{2}, 6$ & $14: 00$ & 7.167 & 133.3 & 124.1 & 125.3 & 119.4 & 118.2 & 118.1 & 116.1 & 109.2 & 111.8 & 86.7 & 83.8 & 81.4 & 107.0 & 89.6 & 86.3 & & & \\
\hline
\end{tabular}


Table 5. Tensiometer Data

\begin{tabular}{|c|c|c|c|c|c|c|c|c|c|c|c|c|c|c|c|c|c|c|c|c|}
\hline & & & & & & & & & Tens & iometer 1 & Data & & & & & & & & & \\
\hline & & Test & $\begin{array}{c}\text { Soll } \\
\text { Moisture } \\
\text { Tension } \\
\left(\mathrm{em} \mathrm{H}_{2} \mathrm{O}\right) \\
\end{array}$ & $\begin{array}{c}\text { Soll } \\
\text { Molsture } \\
\text { Tension } \\
\left(\mathrm{Cm} \mathrm{H}_{2} \mathrm{O}\right)\end{array}$ & $\begin{array}{c}\text { Soil } \\
\text { Moisture } \\
\text { Tension } \\
\left(\mathrm{Cm} \mathrm{H}_{2} \mathrm{O}\right) \\
\end{array}$ & $\begin{array}{c}\text { Soll } \\
\text { Molsture } \\
\text { Tension } \\
\left(\mathrm{Cm} \mathrm{H}_{2} \mathrm{O}\right) \\
\end{array}$ & $\begin{array}{c}\text { Soll } \\
\text { Moisture } \\
\text { Tonsion } \\
\left(\mathrm{Cm} \mathrm{H}_{2} \mathrm{O}\right)\end{array}$ & $\begin{array}{c}\text { Soll } \\
\text { Molsture } \\
\text { Tension } \\
\left(\mathrm{Cm} \mathrm{H}_{2} \mathrm{O}\right) \\
\end{array}$ & $\begin{array}{c}\text { Soll } \\
\text { Moisture } \\
\text { Tension } \\
\left(\mathrm{Cm} \mathrm{H}_{2} \mathrm{O}\right) \\
\end{array}$ & $\begin{array}{c}\text { Soll } \\
\text { Moisture } \\
\text { Tenslon } \\
\left.\text { (cm } \mathrm{H}_{2} \mathrm{O}\right) \\
\end{array}$ & $\begin{array}{c}\text { Soll } \\
\text { Moisture } \\
\text { Tension } \\
\left(\mathrm{Cm} \mathrm{H}_{2} \mathrm{O}\right) \\
\end{array}$ & $\begin{array}{c}\text { Soll } \\
\text { Molsture } \\
\text { Tension } \\
\left(\mathrm{Cm} \mathrm{H}_{2} \mathrm{O}\right) \\
\end{array}$ & $\begin{array}{c}\text { Soll } \\
\text { Molsture } \\
\text { Tension } \\
\left(\mathrm{Cm} \mathrm{H} \mathrm{H}_{2} \mathrm{O}\right) \\
\end{array}$ & $\begin{array}{c}\text { Soll } \\
\text { Molsture } \\
\text { Tenstion } \\
\left(\mathrm{Cm} \mathrm{H}_{2} \mathrm{O}\right) \\
\end{array}$ & $\begin{array}{c}\text { Soll } \\
\text { Molsture } \\
\text { Tonsion } \\
\left.\text { (Cm } \mathrm{H}_{2} \mathrm{O}\right) \\
\end{array}$ & $\begin{array}{c}\text { Soll } \\
\text { Moisture } \\
\text { Tenslon } \\
\left.\text { (em } \mathrm{H}_{2} \mathrm{O}\right) \\
\end{array}$ & $\begin{array}{c}\text { Soll } \\
\text { Moisture } \\
\text { Tension } \\
\left(\mathrm{Cm}^{\left.\mathrm{H}_{2} \mathrm{O}\right)}\right. \\
\end{array}$ & $\begin{array}{c}\text { soll } \\
\text { Moisture } \\
\text { Tenslon } \\
\left(\mathrm{cm} \mathrm{H}_{2} \mathrm{O}\right) \\
\end{array}$ & $\begin{array}{c}\text { soll } \\
\text { Molsture } \\
\text { Tonsion } \\
\left(\mathrm{cm} \mathrm{H}_{2} \mathrm{O}\right) \\
\end{array}$ & $\begin{array}{c}\text { Soll } \\
\text { Molsture } \\
\text { Tensalon } \\
\left(\mathrm{Cm} \mathrm{H}_{2} \mathrm{O}\right) \\
\end{array}$ \\
\hline Date & Time & Days' & $C 1^{n}$ & $B 1^{*}$ & $A 1^{\prime \prime}$ & $\mathrm{C2}^{\mathrm{b}}$ & $\mathbf{B 2}^{\mathrm{b}}$ & $A 2^{b}$ & $\mathrm{C} 3^{\mathrm{C}}$ & $\mathrm{B3}^{\mathrm{C}}$ & $\mathrm{AB}^{\mathrm{C}}$ & $\mathrm{CA}^{\mathrm{d}}$ & $B 4^{\mathrm{d}}$ & $\mathrm{AA}^{\mathrm{d}}$ & $C 5^{\circ}$ & $85^{\circ}$ & $A 5^{\circ}$ & $c 6^{\prime}$ & $\mathrm{Bb}^{1}$ & $A 6^{\prime}$ \\
\hline 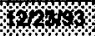 & $16: 00$ & 7.250 & 132.4 & 124.1 & 124.4 & 119.5 & 118.4 & 118.1 & 117.8 & 106.5 & 112.7 & 97.6 & 83.8 & 91.5 & 108.7 & 101.4 & 96.3 & & & \\
\hline 19214 & $18: 00$ & 7.333 & 133.3 & 124.1 & 122.7 & 119.5 & 118.4 & 118.1 & 118.1 & 105.7 & 112.7 & 98.5 & 83.8 & 91.5 & 107.8 & 100.5 & 96.4 & & & \\
\hline 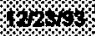 & $20: 00$ & 7.417 & 132.5 & 121.5 & 122.7 & 121.3 & 116.7 & 117.2 & 115.3 & 105.7 & 112.7 & 98.5 & 83.9 & 90.6 & 108.0 & 99.7 & 96.4 & & & \\
\hline $142 \%$ & $22: 00$ & 7.500 & 132.5 & 122.4 & 122.8 & 119.6 & 117.6 & 117.2 & 118.2 & 105.7 & 112.7 & 97.6 & 83.9 & 90.6 & 107.4 & 100.8 & 97.3 & & & \\
\hline $124 \%$ & $0: 00$ & 7.503 & 131.7 & 123.3 & 122.8 & 119.6 & 115.9 & 117.3 & 116.2 & 106.6 & 112.8 & 97.7 & 84.8 & 90.7 & 108.8 & 101.5 & 97.3 & & & \\
\hline $10,0,0$ & $2: 00$ & 7.667 & 432.5 & 124.2 & 124.5 & 121.3 & 114.2 & 117.3 & 115.4 & 104.8 & 112.8 & 97.7 & 85.7 & 92.4 & 108.8 & 101.5 & 98.2 & & & \\
\hline 12240 & $4: 00$ & 7.760 & 133.4 & 125.0 & 123.7 & 120.4 & 115.0 & 117.3 & 118.8 & 105.7 & 112.8 & 98.5 & 86.5 & 93.3 & 108.9 & 101.5 & 97.3 & & & \\
\hline 825010 & $8: 00$ & 7.833 & 133.4 & 123.3 & 123.7 & 120.5 & 120.2 & 118.1 & 117,1 & 105.7 & 114.5 & 99.4 & 86.5 & 93.3 & 108.9 & 101.6 & 96.5 & & & \\
\hline 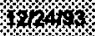 & $8: 00$ & 7.917 & 133.4 & 122.5 & 123.7 & 120.5 & 111.6 & 116.4 & 116.3 & 104.0 & 113.6 & 98.6 & 85.7 & 91.6 & 109.0 & 100.7 & 98.2 & & & \\
\hline 12748 & $10: 00$ & 8.000 & $\$ 34.2$ & 126.8 & 128.2 & 121.3 & 111.6 & 119.9 & 118.0 & 105.7 & 112.7 & 95.9 & 88.2 & 94.1 & 108.0 & 103.2 & 88.2 & & & \\
\hline $1481 \%$ & $12: 00$ & 8.083 & 136.8 & 130.2 & 129.7 & 123.8 & 118.4 & 123.3 & 117.9 & 113.6 & 115.3 & 95.8 & 93.4 & 96.7 & 108.8 & 105.8 & 103.2 & & & \\
\hline $81 \% 4$ & $14: 00$ & 8.167 & 138.4 & 130.1 & 130.5 & 125.5 & 120.9 & 125.0 & 121.2 & 114.4 & 116.1 & 98.4 & 94.1 & 96.7 & 109.5 & 105.8 & 102.3 & $\therefore$ & & \\
\hline$\% 27 \% 8$ & $16: 00$ & 8.250 & 137.5 & 129.1 & 130.4 & 124.5 & 128.6 & 123.2 & 121.1 & 114.4 & 116.9 & 102.7 & 92.3 & 98.6 & 112.0 & 104.8 & 102.1 & & & \\
\hline 42414 & $18: 00$ & 8.333 & 135.8 & 127.5 & 127.0 & 122.9 & 125.2 & 121.5 & 119.5 & 110.8 & 116.1 & 101.8 & 89.8 & 24.9 & 112.1 & 103.9 & 100.5 & & & \\
\hline 81240 & $20: 00$ & 8.417 & 135.8 & 127.6 & 126.2 & 123.8 & 127.9 & 120.7 & 118.8 & 109.2 & 196.2 & 102.0 & 88.2 & 95.0 & 111.4 & 103.2 & 99.0 & & & \\
\hline 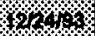 & $22: 00$ & 0.500 & 136.0 & 125.0 & 127.1 & 123.0 & 119.3 & 120.7 & 119.7 & 109.2 & 116.2 & 102.0 & 89.1 & 93.3 & 111.5 & 103.3 & 99.9 & & & \\
\hline $421 \%$ & 0.00 & 8.583 & 135.1 & 127.7 & 126.3 & 123.1 & 115.8 & 120.8 & 119.7 & 108.4 & 118.2 & 102.8 & 88.3 & 95.0 & 111.5 & 104.2 & 99.9 & & & \\
\hline 19149 & $2: 00$ & 8.667 & 136.8 & 125.9 & 125.4 & 124.8 & 121.1 & 121.6 & 119.7 & 109.3 & 117.1 & 102.8 & 89.2 & 95.9 & 112.4 & 104.2 & 99.9 & & & \\
\hline $182 \% 54$ & $4: 00$ & 8.750 & 135.2 & 125.9 & 125.4 & 123.1 & 119.4 & 121.7 & 119.8 & 108.4 & 117.1 & 102.1 & 89.2 & 95.1 & 112.4 & 103.3 & 100.8 & & & \\
\hline 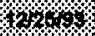 & $6: 00$ & 8.833 & 136.0 & 126.8 & 126.3 & 123.1 & 124.6 & 121.7 & 120.8 & 110.2 & 117.1 & 102.9 & 90.9 & 95.9 & 112.4 & 105.1 & 100.8 & & & \\
\hline $18280 \%$ & 8.00 & 8.917 & 136.1 & 126.8 & 126.3 & 124.9 & 118.0 & 121.7 & 118.9 & 107.5 & 116.3 & 102.1 & 89.2 & 96.8 & 112.5 & 103.4 & 100.9 & & & \\
\hline 122,591 & $10: 00$ & 0.000 & 137.7 & 130.2 & 129.7 & 125.6 & 111.6 & 123.4 & 121.4 & 109.2 & 115.4 & 98.5 & 90.0 & 97.7 & 111.5 & 105.8 & 100.7 & & & \\
\hline $1210 \%$ & $12: 00$ & 0.083 & 138.5 & 132.7 & 134.0 & 128.1 & 109.7 & 125.0 & 120.4 & 111.8 & 117.0 & 97.5 & 95.9 & 100.2 & 110.4 & 108.3 & 104.9 & & & \\
\hline 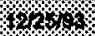 & $14: 00$ & 9.167 & 142.6 & 134.3 & 136.4 & 130.5 & 120.7 & 128.4 & 124.5 & 118.6 & 119.5 & 100.0 & 98.3 & 100.9 & 112.7 & 109.8 & 106.4 & & & \\
\hline 42,58 & $16: 00$ & 2.250 & 141.6 & 134.1 & 136.3 & 131.2 & 136.1 & 128.3 & 127.8 & 122.2 & 123.7 & 106.0 & 88.9 & 103.4 & 117.7 & 109.5 & 107.0 & & & \\
\hline 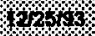 & $18: 00$ & 2.333 & 140.9 & 131.7 & 131.2 & 128.8 & 144.9 & 124.8 & 125.4 & 117.0 & 121.2 & 107.8 & 95.7 & 100.0 & 116.2 & 107.2 & 103.8 & & & \\
\hline $842.4 x$ & $20: 00$ & 0.417 & 140.2 & 131.0 & 128.8 & 128.1 & 128.7 & 124.1 & 123.9 & 1127 & 120.5 & 106.3 & 95.1 & 98.4 & 114.8 & 105.7 & 103.2 & & & \\
\hline $812 \%$ & $22: 00$ & 0.500 & 139.4 & 130.2 & 129.7 & 128.1 & 124.5 & 125.1 & 124.0 & 111.0 & 119.6 & 106.3 & 83.4 & 99.4 & 114.9 & 106.7 & 103.3 & & & \\
\hline 122861 & $0: 00$ & 0.583 & 138.6 & 130.2 & 128.8 & 128.2 & 123.6 & 125.1 & 126.6 & 111.8 & 198.8 & 107.2 & 93.4 & 100.3 & 115.8 & 108.7 & 104.2 & & & \\
\hline 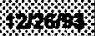 & $2: 00$ & 0.067 & 139.5 & 130.2 & 128.9 & 128.3 & 124.5 & 125.1 & 123.2 & 111.0 & 118.8 & 106.4 & 94.3 & 99.4 & 115.8 & 105.9 & 104.2 & & & \\
\hline 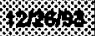 & $4: 00$ & 0.750 & 138.6 & 130.2 & 128.0 & 127.4 & 125.4 & 125.1 & 123.2 & 111.8 & 119.7 & 107.3 & 94.3 & 99.4 & 115.8 & 105.8 & 103.4 & & & \\
\hline $812160 x$ & 8:00 & 0.833 & 138.8 & 130.3 & 128.8 & 127.4 & 125.4 & 125.1 & 124.1 & 112.8 & 120.6 & 107.3 & 94.4 & 100.3 & 115.8 & 107.7 & 105.1 & & & \\
\hline $4 x_{10}$ & $8: 00$ & 0.017 & 139.5 & 130.3 & 128.9 & 126.6 & 120.2 & 125.1 & 122.4 & 111.0 & 119.7 & 107.3 & 93.5 & 99.4 & 115.8 & 105.9 & 104.3 & & & \\
\hline 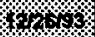 & $10: 00$ & 10.000 & 140.3 & 132.8 & 132.3 & 129.9 & 104.6 & 126.8 & 122.2 & 109.2 & 115.3 & 102.0 & 96.0 & 101.1 & 111.4 & 100.3 & 102.4 & & & \\
\hline 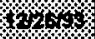 & $12: 00$ & 10.003 & 141.8 & 134,4 & 134.8 & 129.8 & 112.3 & 128.5 & 122.1 & 111.8 & 117.0 & 98.4 & 98.5 & 103.7 & 112.1 & 110.0 & 108.8 & & & \\
\hline $172 \% 19$ & $14: 00$ & 10.107 & 145.3 & 138.9 & 138.2 & 133.2 & 130.3 & 131.0 & 128.0 & 124.1 & 123.0 & 104.4 & 103.5 & 104.5 & 118.2 & 111.6 & 108.1 & & & \\
\hline $824 \% 0$ & $16: 00$ & 10.250 & 1466.1 & 136.9 & 138.2 & 134.9 & 135.4 & 131.9 & 131.4 & 124.1 & 124.7 & 110.5 & 101.8 & 105.3 & 120.5 & 113.3 & 109.8 & & & \\
\hline $812 \mathrm{~s}$ & $18: 00$ & 10.333 & 144.5 & 134.4 & 134.8 & 132.4 & 143.3 & 128.5 & 129.0 & 118.8 & 124.7 & 110.6 & 100.2 & 103.6 & 119.8 & 110.8 & 108.3 & & & \\
\hline
\end{tabular}


Table 5. Tensiometer Data

\begin{tabular}{|c|c|c|c|c|c|c|c|c|c|c|c|c|c|c|c|c|c|c|c|c|}
\hline & & & & & & & & & Ten: & siometer & Jata & & & & & & & & & \\
\hline & & Test & $\begin{array}{c}\text { Soll } \\
\text { Molsture } \\
\text { Tension } \\
\left(\mathrm{Cm} \mathrm{H}_{2} \mathrm{O}\right) \\
\end{array}$ & $\begin{array}{c}\text { Soil } \\
\text { Molsture } \\
\text { Tension } \\
\left(\mathrm{Cm} \mathrm{H}_{2} \mathrm{O}\right) \\
\end{array}$ & $\begin{array}{c}\text { Soil } \\
\text { Molsture } \\
\text { Tension } \\
\left(\mathrm{Cm} \mathrm{H}_{2} \mathrm{O}\right) \\
\end{array}$ & $\begin{array}{c}\text { Soll } \\
\text { Moisture } \\
\text { Tenslon } \\
\left.\text { (cm } \mathrm{H}_{2} \mathrm{O}\right) \\
\end{array}$ & $\begin{array}{c}\text { Soll } \\
\text { Molsture } \\
\text { Tension } \\
\left(\mathrm{C} \mathrm{H}_{2} \mathrm{O}\right) \\
\end{array}$ & $\begin{array}{c}\text { Soll } \\
\text { Moisture } \\
\text { Tension } \\
\left.\text { (cm } \mathrm{H}_{2} \mathrm{O}\right) \\
\end{array}$ & $\begin{array}{c}\text { Soll } \\
\text { Moisture } \\
\text { Tenslon } \\
\left(\mathrm{Cm}_{\left.\mathrm{H}_{2} \mathrm{O}\right)}\right. \\
\end{array}$ & $\begin{array}{c}\text { Soil } \\
\text { Molsture } \\
\text { Tension } \\
\left(\mathrm{cm} \mathrm{H}_{2} \mathrm{O}\right) \\
\end{array}$ & $\begin{array}{c}\text { Soll } \\
\text { Molsture } \\
\text { Tension } \\
\left(\mathrm{Cm}_{2} \mathrm{O}\right) \\
\end{array}$ & 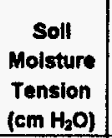 & $\begin{array}{c}\text { Soll } \\
\text { Molsture } \\
\text { Tenston } \\
\left(\mathrm{Cm} \mathrm{H}_{2} \mathrm{O}\right) \\
\end{array}$ & $\begin{array}{c}\text { Soll } \\
\text { Molsture } \\
\text { Tension } \\
\left.\text { (em } \mathrm{H}_{2} \mathrm{O}\right) \\
\end{array}$ & $\begin{array}{c}\text { Soll } \\
\text { Moisture } \\
\text { Tenslon } \\
\left.\text { (cm } \mathrm{H}_{2} \mathrm{O}\right) \\
\end{array}$ & $\begin{array}{c}\text { Soll } \\
\text { Molsture } \\
\text { Tension } \\
\left(\mathrm{Cm} \mathrm{H}_{2} \mathrm{O}\right) \\
\end{array}$ & $\begin{array}{c}\text { Soll } \\
\text { Molsture } \\
\text { Tension } \\
\left(\mathrm{Cm} \mathrm{H}_{2} \mathrm{O}\right) \\
\end{array}$ & $\begin{array}{c}\text { soll } \\
\text { Molsture } \\
\text { Tonsion } \\
\left(\mathrm{Cm} \mathrm{H}_{2} \mathrm{O}\right)\end{array}$ & $\begin{array}{c}\text { Soll } \\
\text { Molsture } \\
\text { Tension } \\
\left(\mathrm{Cm} \mathrm{H}_{2} \mathrm{O}\right)\end{array}$ & $\begin{array}{c}\text { Soll } \\
\text { Moisture } \\
\text { Tension } \\
\left(\mathrm{em} \mathrm{H}_{2} \mathrm{O}\right) \\
\end{array}$ \\
\hline Date & Time & Days' & $C 1^{n}$ & $B 1^{\prime \prime}$ & $A 1^{\prime \prime}$ & $\mathrm{C2}^{6}$ & $\mathrm{B2}^{\circ}$ & $A 2^{6}$ & $C 3^{\circ}$ & $\mathrm{B}^{\mathrm{C}}$ & $A 3^{\circ}$ & $\mathrm{CA}^{\mathrm{d}}$ & $B 4^{\mathrm{d}}$ & $\mathbf{A 4}^{d}$ & $C 5^{\circ}$ & $85^{\circ}$ & $A 5^{\circ}$ & $\cos ^{\prime}$ & $86^{\circ}$ & $A 6^{\prime}$ \\
\hline 1. 60 & $20: 00$ & 10.417 & 143.7 & 134.5 & 133.1 & 131.6 & 133.9 & 128.5 & 128.2 & 118.8 & 123.1 & 109.8 & 99.4 & 104.6 & 118.2 & 110.1 & 108.7 & & & \\
\hline 172,15 & $22: 00$ & 10.600 & 143.7 & 434,5 & 133.2 & 131.6 & 128.7 & 128.5 & 128.3 & 117.1 & 123.1 & 108.9 & 89.4 & 103.7 & 119.1 & 110.1 & 108.7 & & & \\
\hline 1427\% & $0: 00$ & 10.583 & 143.7 & 134.5 & 134.1 & 131.7 & 128.8 & 127.7 & 128.3 & 116.2 & 123.1 & 109.8 & 89.5 & 103.7 & 118.3 & 110.1 & 100.7 & & & \\
\hline $12, \% 4$ & $2: 00$ & 10.667 & 143.8 & 134.5 & 133.2 & 133.4 & 129.6 & 130.3 & 128.3 & 117.1 & 123.1 & 110.7 & 89.5 & 103.7 & 118.3 & 110.2 & 108.7 & & & \\
\hline $22 \% 7 \%$ & $4: 00$ & 10.750 & 143.8 & 134.5 & 133.2 & 131.7 & 123.6 & 128.8 & 128.6 & 118.3 & 123.1 & 108.9 & 99.5 & 102.8 & 118.3 & 110.2 & 108.8 & & & \\
\hline 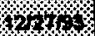 & $6: 00$ & 10.833 & 143.8 & 134.5 & 133.2 & 131.7 & 128.7 & 128.8 & 130.1 & 117.2 & 124.0 & 110.7 & 89.5 & 103.7 & 120.9 & 110.2 & 108.5 & & & \\
\hline 4274 & $8: 00$ & 10.917 & 143.8 & 134.5 & 133.2 & 132.6 & 125.3 & 128.6 & 126.6 & 115.4 & 123.1 & 109.0 & 100.4 & 104.6 & 118.2 & 111.1 & 107.6 & & & \\
\hline 425 & $10: 00$ & 11.000 & 144.6 & 138.0 & 136.6 & 134.2 & 118.2 & 132.0 & 126.5 & 116.2 & 122.2 & 106.3 & 1020 & 106.3 & 117.4 & 112.7 & 109.3 & & & \\
\hline $101.8 \%$ & $12: 00$ & 11.083 & 145.4 & 138.7 & 138.3 & 135.8 & 116.6 & 132.8 & 129.0 & 118.8 & 222.1 & 104.5 & 103.6 & 107.1 & 116.4 & 115.2 & 110.9 & & & \\
\hline $12+1,13$ & $14: 00$ & 11.167 & 147.1 & 138.7 & 140.0 & 136.7 & 126.8 & 134.5 & 129.8 & 121.4 & 124.7 & 107.9 & 105.3 & 107.1 & 120.6 & 114.2 & 111.7 & & & \\
\hline $142 \%$ & $16: 00$ & 11.250 & 147.9 & 139.8 & 139.1 & 135.8 & 140.7 & 135.4 & 134.1 & 127.6 & 128.2 & 114.0 & 108.2 & 108.0 & 123.2 & 115.1 & 112.5 & & & \\
\hline 24749 & $18: 00$ & 11.333 & 147.1 & 137.0 & 137.4 & 135.8 & 141.6 & 132.0 & 932.5 & 124.1 & 128.2 & 114.1 & 104.5 & 107.1 & 123.3 & 113.5 & 111.7 & $\therefore$ & & \\
\hline 62674 & $20: 00$ & 11.417 & 148.0 & 137.9 & 137.5 & 136.8 & 135.8 & 133.7 & 131.8 & 123.2 & 126.5 & 112.3 & 104.6 & 108.0 & 122.5 & 113.5 & 111.8 & & & \\
\hline $2,2, \%$ & $22: 00$ & 11.500 & 147.2 & 137.9 & 137.5 & 136.8 & 136.5 & 132.9 & 131.7 & 122.4 & 125.7 & 113.2 & 104.8 & 108.0 & 122.5 & 113.5 & 111.0 & & & \\
\hline 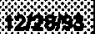 & $0: 00$ & 11.583 & 147.2 & 137.1 & 137.5 & 136.8 & 130.4 & 132.9 & 131.7 & 121.5 & 126.5 & 112.4 & 103.7 & 108.1 & 121.7 & 113.6 & 111.8 & & & \\
\hline 422280 & $2: 00$ & 11.667 & 147.2 & 138.0 & 136.6 & 136.8 & 133.0 & 133.7 & 131.7 & 122.4 & 126.5 & 115.0 & 105.5 & 108.1 & 123.4 & 114.4 & 111.8 & & & \\
\hline 1. & 4:00 & 11.750 & 147.2 & 138.0 & 136.7 & 136.9 & 132.2 & 132.8 & 131.7 & 121.5 & 126.6 & 113.3 & 103.8 & 108.1 & 123.5 & 114.5 & 111,8 & & & \\
\hline (10.6. & $8: 00$ & 11.833 & 147.3 & 138.0 & 135.8 & 136.8 & 134.8 & 132.9 & 131.8 & 124.2 & 128.3 & 115.9 & 103.8 & 108.1 & 125.3 & 113.7 & 111.8 & & & \\
\hline $1028=4$ & $8: 00$ & 11.917 & 147.3 & 138.0 & 135.8 & 136.8 & 124.5 & 132.9 & 131.8 & 118.9 & 128.6 & 116.0 & 103.9 & 108.1 & 124.4 & 113.7 & 112.0 & & & \\
\hline 1248,1 & $10: 00$ & 12.000 & 146.3 & 138.0 & 137.5 & 136.9 & & 132.8 & 128.3 & 110.9 & 118.7 & 108.9 & 103.8 & 109.0 & 118.3 & 113.6 & 1009.3 & & & \\
\hline 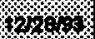 & \begin{tabular}{|l|}
$12: 00$ \\
\end{tabular} & 12.083 & 145.3 & 141.3 & 141.8 & 139.3 & & 135.4 & 129.8 & 117.9 & 121.3 & 102.7 & 108.2 & 110.8 & 118.9 & 915.2 & 115.1 & & & \\
\hline 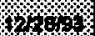 & $14: 00$ & 12.167 & 156.5 & 138.6 & 146.0 & 143.6 & 132.8 & 141.5 & 138.4 & 137.3 & 132.5 & 112.2 & 112.2 & 114.9 & 124.8 & 123.7 & 118.3 & 132,2 & & \\
\hline 422.86 & 18:00 & 12.250 & 150.4 & 139.5 & 142.5 & 140.1 & 145.7 & 137.1 & 138.3 & 134.6 & 133.3 & 116.8 & 110.4 & 112.3 & 127.4 & 117.6 & 116.7 & 145.3 & & \\
\hline 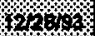 & $18: 00$ & 12.333 & 150.5 & 139.8 & 140.0 & 140.2 & 147.6 & 136.3 & 136.8 & 131.1 & 135.1 & 119.3 & 108.8 & 110.6 & 128.4 & 116.8 & 115.1 & 150.7 & & \\
\hline \% & $20: 00$ & 12.417 & 148.9 & 138.8 & 139.3 & 139.5 & 139.1 & 133.7 & 134.3 & 123.3 & 130.0 & 117.8 & 106.4 & 100.8 & 125.2 & 118.2 & 114.4 & 148.3 & & \\
\hline \% & $22: 00$ & 12.500 & 148.1 & 138.0 & 138.4 & 139.5 & 128.8 & 134.6 & 132.8 & 121.5 & 129.2 & 115.8 & 107.3 & 110.7 & 125.2 & 117.2 & 113.7 & 145.8 & & \\
\hline 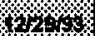 & $0: 00$ & 12.583 & 149.9 & 138.9 & 139.3 & 140.4 & 123.6 & 135.5 & 133.5 & 123.3 & 130.0 & 116.8 & 105.8 & 110.7 & 125.2 & 117.2 & 113.7 & 145.8 & & \\
\hline 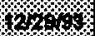 & $2: 00$ & 12.667 & 149.8 & 140.6 & 139.3 & 140.4 & 128.8 & 136.4 & 135.3 & 128.8 & 131.8 & 117.7 & 107.3 & 1111.6 & 127.8 & 117.2 & 113.7 & 148.4 & & \\
\hline $4 \%$ & 4:00 & 12.750 & 148.1 & 139.8 & 138.5 & 138.7 & 128.2 & 133.8 & 135.3 & 123.3 & 130.1 & 118.6 & 108.5 & 110.8 & 128.2 & 116.3 & 113.7 & 149.3 & & \\
\hline 42,94 & $6: 00$ & 12.833 & 149.0 & 139.8 & 138.5 & 139.8 & 127.1 & 135.6 & 133.6 & 123.4 & 130.1 & 117.7 & 100.5 & 111.7 & 127.1 & 115.5 & 115.5 & 149.4 & & \\
\hline \% & 8.00 & 12.0917 & 149.0 & 139.8 & 138.5 & 139.8 & 123.7 & 135.6 & 135.3 & 123.4 & 130.1 & 117.7 & 107.4 & 110.8 & 128.2 & 117.2 & 115.5 & 148.4 & & \\
\hline \% & $10: 00$ & 13.000 & 147.2 & 141.5 & 940.2 & 139.5 & & 138.4 & 130.8 & 112.7 & 121.4 & 112.4 & 107.3 & 111.6 & 121.8 & 117.2 & 112.8 & 141.4 & & \\
\hline (2) 290 & $12: 00$ & 13.083 & 150.8 & 144.0 & 144.4 & 1420 & & 138.9 & 129.9 & 122.4 & 124.7 & 107.1 & 111.5 & 114.2 & 121.6 & 121.3 & 118.6 & 133.3 & & \\
\hline 102.48 & 14:00 & 13.167 & 155.7 & 139.5 & 146.9 & 145.3 & 121.7 & 121.4 & 140.2 & 134.7 & 131.6 & 114.8 & 115.7 & 116.7 & 128.6 & 122.8 & 121.1 & 143.6 & & \\
\hline 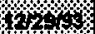 & $18: 00$ & 13.250 & 155.7 & 141.2 & 146.1 & 144.4 & 138.9 & 117.1 & 141.8 & 139.0 & 139.4 & 119.2 & 113.9 & 115.8 & 131.8 & 122.0 & 120.2 & 154.1 & & \\
\hline 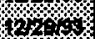 & $18: 00$ & 13.333 & 152.3 & 143.1 & 142.7 & 142.8 & 143.3 & 114.5 & 140.3 & 135.6 & 138.6 & 121.0 & 112.3 & 114.1 & 131.8 & 119.6 & 1177.8 & 156.0 & & \\
\hline 2729 & $20: 00$ & 13.417 & 150.7 & 141.4 & 140.2 & 143.0 & 138.5 & 111.9 & 137.8 & 127.7 & 133.5 & 120.3 & 109.9 & 112.5 & 128.6 & 118.0 & 116.2 & 152.7 & & \\
\hline 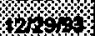 & $22: 00$ & 13.500 & 150.7 & 141.5 & 141.1 & 142.1 & 132.2 & 110.2 & 137.8 & 128.8 & 933.5 & 119.4 & 110.8 & 110.7 & 128.7 & 118.9 & 117.1 & 151.8 & & \\
\hline
\end{tabular}




\begin{tabular}{|c|c|c|c|c|c|c|c|c|c|c|c|c|c|c|c|c|c|c|c|c|}
\hline & & |'8S1 & 1226 & FSIL & $90 \mathrm{gl}$ & S'61t & 1916 & 7021 & $9^{\circ} 6 \varepsilon 1$ & IEहL & Optol & & & 00 OSI & 1801 & 9871 & S'BSI & $\angle 0091$ & $00: 2$ & $617 \%$ \\
\hline & & I'8St & 1221 & 1921 & 8 tVL & S6Lt & 0.916 & $\varepsilon \cdot \angle Z L$ & 9001 & $6 \varepsilon \varepsilon 1$ & 8601 & & & O'OSL & $z \angle t L$ & $S^{\prime} 8016$ & S'6SL & $809^{\circ} 9 t$ & $00: 0$ & $14 \%$ \\
\hline & & O'OSt & S'LI & 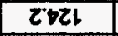 & 8 VEl & 98416 & 0.946 & 2821 & sort & LSEL & 8601 & & $\nabla \mid \varepsilon L$ & OOOSI & 1811 & $9 \angle 01$ & TESL & $\cos 91$ & $\infty: 2 \pi$ & 146 \\
\hline & & 9001 & $68 Z 1$ & 6721 & $8 \varepsilon 1$ & 6646 & $2 \angle 41$ & 1.821 & O'Eका & 0001 & 9'sth & & $8.68 L$ & 8.051 & I.LDL & 6806 & D'6SL & $\angle A+9 t$ & $\infty$ & 14 \\
\hline & & 9'691 & $9 \cdot 26$ & S'SZL & 9081 & 1.121 & 2616 & S'IEL & Losi & $\varepsilon \mathrm{zSt}$ & $90 \mathrm{OSL}$ & & 9 bel & EZst & 2801 & 6671 & 92.9l & $\varepsilon \varepsilon \varepsilon y \mathrm{I}$ & $\infty: 81$ & 14 \\
\hline & & I'ESL & 6926 & $9^{\prime} 62 t$ & S'BEL $^{\prime}$ & $8 \varepsilon Z 1$ & $8 \pi 2$ & 0.221 & $9 \mathrm{EDl}$ & $8 \angle \mathrm{BL}$ & Soth & & & 8'ES1 & B'ESL & bsth & I'S9l & $052 \% 1$ & $\infty$ & $6 \%$ \\
\hline & & 6.871 & 8921 & 1021 & $9 \varepsilon \varepsilon 1$ & 1.121 & 2861 & 8616 & ISEl & $\angle$ tot & stol & & & ZESI & ZZSL & 2871 & $\angle 291$ & $\angle 9191$ & $\infty 0: 0$ & $61 \%$ \\
\hline & & 7001 & 9'SZL & $\tau 6 z t$ & 8.821 & $\varepsilon^{\prime} 0 z 1$ & 7646 & 6216 & 8121 & $\angle 6 L 6$ & $\varepsilon \forall \varepsilon b$ & & & 0601 & EZSL & $\varepsilon 8+1$ & LOSL & $880^{\circ} 91$ & $00: 26$ & $16 \%$ \\
\hline & & $L 2+1$ & 8611 & 2021 & $\angle 821$ & 2816 & ZSH & 9816 & 8121 & 8916 & ILEL & & & $7 \angle O L$ & $2 \angle D L$ & $8 . \mathrm{StL}$ & $\varepsilon t s t$ & $000 \% 1$ & $\infty 0: 06$ & $16 \%$ \\
\hline & & LSSL & $8^{\prime} 0 z 6$ & 9221 & l'tel & 0.216 & L'ZH & $8.62 b$ & Z'0६1 & L'BZL & 9001 & & & $S^{\prime} \angle t L$ & LOtL & D'9th & Z'SSL & 46691 & $00: 8$ & 414 \\
\hline & & $7 \angle S I$ & 9126 & 9221 & I'tel & $8 \angle 14$ & 2211 & $9 \mathrm{sZl}$ & 0.861 & S081 & stot & & & 9.901 & 2001 & 8901 & ZSSL & $8 \varepsilon 891$ & $00: 9$ & 4 \\
\hline & & 2851 & 9121 & $5 \varepsilon 21$ & $6.6 \varepsilon 1$ & 82111 & toth & s.9zt & L'GEL & $\varepsilon^{\prime}|\varepsilon|$ & $\varepsilon z+1$ & & ZहEL & $5 \angle D L$ & 9001 & 8.901 & 0.851 & 05191 & $00 ; 0$ & 14 \\
\hline & & TOSL & 1226 & $\varepsilon \varepsilon z L$ & $8 \mathrm{VEl}$ & 6911 & EDLL & $\angle 2 t Z t$ & $8^{\prime} 8 \varepsilon 1$ & $\varepsilon^{\prime}|\varepsilon|$ & Flth & & tiEk & $7 \angle O L$ & SSPL & $2.9+1$ & 6.991 & 20996 & 00.2 & $14 \%$ \\
\hline & & I.8SL & 9021 & $\varepsilon \varepsilon z t$ & 8'VEL & 6911 & $\varepsilon+4 t$ & 7921 & $28 \varepsilon 1$ & ZZદト & $2 z+1$ & & ZZEt & $T \angle P H$ & 9.01 & $8 \mathrm{sth}$ & $8 \% \mathrm{SL}$ & 88991 & $00: 0$ & 1411 \\
\hline & & 6.891 & SOZL & $1 \Rightarrow 21$ & L'BEl & $2 \angle L 1$ & $2+16$ & $2 \angle 21$ & 7001 & 6E\&L & $0 \mathrm{EDL}$ & & S'get & 2801 & S'tol & $8 \mathrm{gth}$ & $\theta \cdot 2 \mathrm{SL}$ & $009^{9} 91$ & $\overline{00: 2 z}$ & \%4.1. \\
\hline & & L'OSL & $12 Z 1$ & 0021 & 9. & 5811 & $0 \mathrm{sLl}$ & 8921 & $12 p \mid$ & $\varepsilon \dot{L} \angle \mathrm{EL}$ & $8 \mathrm{EPl}$ & & $0.6 \varepsilon 1$ & 1801 & 2901 & $2 Z+1$ & $9 \angle S L$ & $\angle b-96$ & $00: 0 z$ & 14 \\
\hline & & 9691 & LधZL & $\angle \nabla Z L$ & $0<\varepsilon \varepsilon 1$ & $\varepsilon 6 t 1$ & 9.941 & $90 \varepsilon 1$ & $\tau^{\prime} \angle D L$ & $0<01$ & 0801 & & $6.8 \varepsilon b$ & 8801 & $8 \angle \mathrm{Lb}$ & $6 \varepsilon p h$ & Z6SL & $\varepsilon \varepsilon \varepsilon^{\prime} 9 l$ & $00: 81$ & $1,1,1,2=$ \\
\hline & & LSSI & 2921 & $1 \angle Z 1$ & 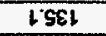 & 6.121 & $661 \mathrm{t}$ & SEZt & $8 \mathrm{ZPl}$ & 1 sol & 6.901 & & & tost & FISL & 9006 & szol & 05296 & $00: 91$ & 1014 \\
\hline & & SsDL & $Z^{\prime \prime}|Z|$ & $\angle D Z L$ & 8621 & $8 \angle 46$ & 8946 & 6016 & 6.621 & 5821 & $D^{\prime} 6 \varepsilon L$ & & & 6.801 & 2801 & 9801 & S'LSL & $\angle 0596$ & $\infty$ & $18 \%$ \\
\hline & & 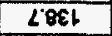 & $\varepsilon 1 Z L$ & $0 D Z L$ & $\varepsilon \nabla 21$ & $2 \angle L L$ & 2741 & s.th & $68 Z 1$ & 1216 & TहEl & & & $\varepsilon \angle D L$ & 2601 & $S \angle D L$ & $\varepsilon \varepsilon S L$ & 68096 & $00: 21$ & 1.116 .1 \\
\hline & & 8276 & $2 \angle 61$ & $\angle 0 Z 1$ & $\varepsilon \mathrm{sZl}$ & 0961 & 8041 & szth & $\varepsilon z Z 1$ & LZLI & 8'LEL & & & 81601 & SSOL & 0.901 & 80 OSL & 000951 & 00.01 & $181,1 \%$ \\
\hline & & 0691 & 5846 & 6616 & 9081 & $\varepsilon \nabla H$ & 8416 & 2621 & strel & 6.921 & LEEl & & & LSPL & $8 \varepsilon+1$ & 0.901 & $\varepsilon \forall S L$ & $\angle 162 b$ & $00: 8$ & 264,8 \\
\hline & & ZLSL & 6846 & 0.616 & 9081 & ZSLL & 8.46 & S6LI & tमEl & 0.921 & 8 '8EL & & & LSbl & 0.201 & $0, s+1$ & DESL & $E E B^{\prime \prime H}$ & $00 ; 8$ & 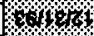 \\
\hline & & 8'ESt & 0616 & $\angle O Z L$ & s.0EL & Zstl & 2111 & 1221 & $7 t E 1$ & 6.921 & $\angle B E 1$ & & & $2 s p 1$ & $62 D 1$ & $0.9 D 6$ & $\varepsilon \mathrm{BSL}$ & OSLH & $00: 0$ & 1407 \\
\hline & & ZISI & 1.846 & 0616 & LEZL & 871 & 0046 & 8021 & DVEI & 6921 & 6'LEL & & & 6.201 & 0201 & $\varepsilon \varepsilon+1$ & szst & $\angle 0976$ & 00.2 & 1018.2 \\
\hline & & 6ZSL & 2446 & 0611 & SOEL & $\varepsilon \nabla H$ & 00011 & 2121 & $7 \forall E 1$ & 6.921 & 8.881 & & & 801 & 0201 & टहDi & DESL & 8897 & $00: 0$ & 101,1 \\
\hline & & $82 \mathrm{CSL}$ & T2L1 & $\angle O Z L$ & SOEL & $\varepsilon \nabla L$ & sZLL & $12 z 1$ & EGEI & $\angle \angle Z L$ & $18 \varepsilon 1$ & & & 8006 & 8201 & OSPL & $\nabla E S L$ & $009^{2} 11$ & 00.22 & $16 \%$ \\
\hline & & Z9St & TLLL & 9.071 & |zE। & ISLL & g'tht & 6227 & $\angle 8 E 1$ & $\tau '|\varepsilon|$ & $S^{\prime} 6 \varepsilon \downarrow$ & & LSt & $2+16$ & $82+6$ & $00+66$ & $\varepsilon \varepsilon S L$ & 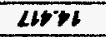 & $00: 02$ & $160 \times 1$ \\
\hline & & 1281 & ILIZL & 0.221 & $S \varepsilon \varepsilon 1$ & $\angle 941$ & 8716 & 2.921 & 2801 & $\varepsilon 60 t$ & $9 \mathrm{EDL}$ & 7201 & O'LSI & 2901 & $E D D L$ & 9'sth & $995 \mathrm{~L}$ & $E E E D L$ & 00.81 & $860 \times 1$ \\
\hline & & LtSL & 9271 & $9 \varepsilon Z 1$ & $\varepsilon \varepsilon \varepsilon \downarrow$ & $E 84$ & $2 \angle 16$ & 92221 & 6.101 & 0.9016 & Z'StL & 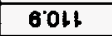 & $\angle 8 \varepsilon 1$ & $8 \mathrm{LOb}$ & $9.8+1$ & $\angle \varepsilon+L$ & 0.651 & 09276 & $00: 96$ & 1840,1 \\
\hline & & $2 \mathrm{EDL}$ & $1.12 L$ & $08 Z 1$ & $s<21$ & $\angle 911$ & $2 \mathrm{SH}$ & 0011 & Q'LI & 6281 & $\varepsilon 6 \varepsilon l$ & O.tol & & 2901 & $\angle 8 D L$ & sotl & 9951 & $\angle 9171$ & 00.12 & 464 \\
\hline & & 6'961 & E'LLL & $\varepsilon \approx 21$ & szZl & 8941 & 7211 & $8^{\prime} 601$ & $862 b$ & 9021 & L'LL & $1 \angle B$ & & ssth & 0.816 & 9.9016 & tZSL & $880 \mathrm{th}$ & $00: 26$ & 04018 \\
\hline & & 8501 & LELL & 8614 & 8921 & $E D L$ & 1601 & 2011 & $\varepsilon z Z 1$ & 2216 & $9 \varepsilon \varepsilon$ & 688 & & 6801 & $82+6$ & $D z+L$ & 8051 & 00076 & 0000 & 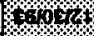 \\
\hline & & $\varepsilon^{\prime}|S|$ & 206 & 2861 & 6.821 & 8011 & $\varepsilon 80 \mathrm{~L}$ & S'6!t & $0.1 \varepsilon \mid$ & 8'LZL & 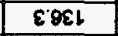 & 2001 & & 7101 & 5686 & 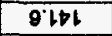 & OOOSL & $416 \mathrm{EL}$ & $00: 8$ & 40114 \\
\hline & & E'LS & 9S16 & 1616 & $\angle B Z 1$ & 8211 & 2601 & 7021 & ZZEL & ZSZL & b'LE & 6.9OL & & 8201 & $02+1$ & szol & LISL & $E E B E B$ & $00: 8$ & $16 \%, 1$ \\
\hline & & Z'St & S'sth & 1616 & 0.821 & LIL & 2601 & S6Lt & LZE। & $2 \mathrm{SZ1}$ & 2066 & 9901 & & 2201 & $76 \varepsilon 6$ & 2006 & 80 .0St & 09281 & $00: 1$ & 101014 \\
\hline & & 0891 & SGl6 & 0.811 & 8821 & LIL & 2601 & solt & $81 E 1$ & I'szi & T'LEL & 8201 & $\angle G Z L$ & 2201 & 6681 & 7206 & 8096 & $\angle 99 E b$ & $00: 2$ & $160 \times 6$ \\
\hline & & O'ZSL & $\varepsilon \% 16$ & 0.616 & 8.821 & $2+16$ & $7 \mathrm{LOL}$ & S61t & $9 \varepsilon \varepsilon 1$ & 0.021 & ILEL & 7601 & $\varepsilon z \varepsilon t$ & ELtol & 2001 & sitht & LISL & 68961 & $00: 0$ & 3640 \\
\hline 98 & 98 & 90 & .98 & .98 & 90 & $p$ & 58 & $\mathrm{p} 75$ & हEV & 589 & .80 & $a^{20}$ & 98 & .20 & .10 & .18 & .15 & .5Keg & - & गेea \\
\hline 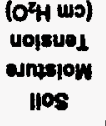 & 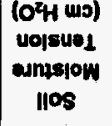 & 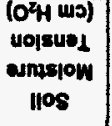 & $\begin{array}{c}\mathrm{O}^{2} \mathrm{H} \text { WD) } \\
\text { volsuol } \\
\text { andssiow } \\
\| 10 \mathrm{~S}\end{array}$ & $\begin{array}{c}0^{z} H \text { wD } \\
\text { uo|suol } \\
\text { umpajow } \\
\| 08\end{array}$ & 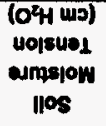 & 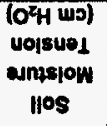 & $\begin{array}{c}0^{5} \mathrm{H} \text { WJ) } \\
\text { Molswol } \\
\text { ansssiow } \\
\| 10 S\end{array}$ & 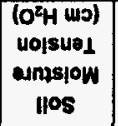 & 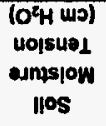 & 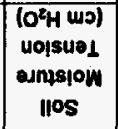 & 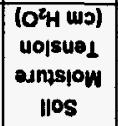 & 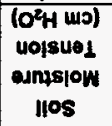 & 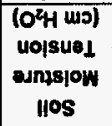 & 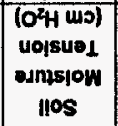 & $\begin{array}{c}\text { (ofH wo) } \\
\text { uojsue } \\
\text { eanus|ow } \\
\text { nos }\end{array}$ & 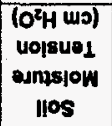 & 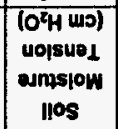 & 2501 & & \\
\hline & & & & & & & & & \multicolumn{3}{|c|}{ 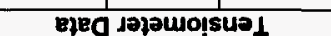 } & & & & & & & & & \\
\hline
\end{tabular}


Table 5. Tensiometer Data

\begin{tabular}{|c|c|c|c|c|c|c|c|c|c|c|c|c|c|c|c|c|c|c|c|c|}
\hline & & & & & & & & & Ten: & iometer & Sata & & & & & & & & & \\
\hline & & Test & $\begin{array}{c}\text { Soil } \\
\text { Molsture } \\
\text { Tension } \\
\left(\mathrm{Cm} \mathrm{H}_{2} \mathrm{O}\right) \\
\end{array}$ & $\begin{array}{c}\text { Soll } \\
\text { Molsture } \\
\text { Tension } \\
\left(\mathrm{Cm} \mathrm{H}_{2} \mathrm{O}\right) \\
\end{array}$ & $\begin{array}{c}\text { Soll } \\
\text { Molsture } \\
\text { Tension } \\
\left(\mathrm{Cm} \mathrm{H}_{2} \mathrm{O}\right) \\
\end{array}$ & 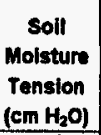 & $\begin{array}{c}\text { Soll } \\
\text { Molsture } \\
\text { Tension } \\
\left(\mathrm{Cm}_{2} \mathrm{O}\right)\end{array}$ & 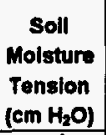 & $\begin{array}{c}\text { Soll } \\
\text { Moisture } \\
\text { Tension } \\
\left(\mathrm{Cm} \mathrm{H}_{2} \mathrm{O}\right)\end{array}$ & $\begin{array}{c}\text { Soll } \\
\text { Molsture } \\
\text { Tension } \\
\left(\mathrm{Cm} \mathrm{H}_{2} \mathrm{O}\right)\end{array}$ & $\begin{array}{c}\text { Soll } \\
\text { Moisture } \\
\text { Tenslon } \\
\left.\text { (cm } \mathrm{H}_{2} \mathrm{O}\right) \\
\end{array}$ & $\begin{array}{c}\text { Soll } \\
\text { Molsture } \\
\text { Tension } \\
\left(\mathrm{Cm} \mathrm{H}_{2} \mathrm{O}\right) \\
\end{array}$ & $\begin{array}{c}\text { Soll } \\
\text { Moisture } \\
\text { Tension } \\
\left(\mathrm{Cm} \mathrm{H}_{2} \mathrm{O}\right) \\
\end{array}$ & $\begin{array}{c}\text { Soll } \\
\text { Moisture } \\
\text { Tension } \\
\left(\mathrm{Cm}_{2} \mathrm{O}\right) \\
\end{array}$ & $\begin{array}{c}\text { Soll } \\
\text { Moisture } \\
\text { Tension } \\
\left(\mathrm{Cm} \mathrm{H}_{3} \mathrm{O}\right) \\
\end{array}$ & $\begin{array}{c}\text { Soll } \\
\text { Molsture } \\
\text { Tension } \\
\left(\mathrm{Cm} \mathrm{H}_{2} \mathrm{O}\right) \\
\end{array}$ & $\begin{array}{c}\text { Soll } \\
\text { Moisture } \\
\text { Tension } \\
\text { (em HeO) }\end{array}$ & $\begin{array}{c}\text { soll } \\
\text { Molsture } \\
\text { Tension } \\
\left(\mathrm{Cm} \mathrm{H} \mathrm{H}_{2} \mathrm{O}\right)\end{array}$ & $\begin{array}{c}\text { Soll } \\
\text { Molsture } \\
\text { Tenslon } \\
\left(\mathrm{Cm} \mathrm{H}_{2} \mathrm{O}\right)\end{array}$ & $\begin{array}{c}\text { soll } \\
\text { Moisture } \\
\text { Tension } \\
\left(\mathrm{Cm} \mathrm{H}_{2} \mathrm{O}\right)\end{array}$ \\
\hline Date & Time & Days' & $\mathrm{C1}^{*}$ & $B 1^{\prime \prime}$ & $A 1^{\prime \prime}$ & $\mathbf{C 2}^{\mathbf{b}}$ & $B 2^{6}$ & $A 2^{b}$ & $\mathrm{C3}^{\mathrm{C}}$ & $B 3^{\circ}$ & $\mathrm{A}^{\mathrm{C}}$ & $\mathrm{C}^{\mathrm{d}}$ & $B 4^{d}$ & $A 4^{d}$ & $C 5^{\circ}$ & $\mathrm{B5}^{\circ}$ & $A 5^{\circ}$ & $c 6^{\prime}$ & $B 6^{f}$ & $A B^{\prime}$ \\
\hline $10 \%$ & $4: 00$ & 16.760 & 159.5 & 147.6 & 147.2 & 150.8 & & & 145.7 & 134.0 & 140.5 & 128.2 & 116.1 & 119.6 & 136.6 & 126.0 & 124.1 & 158.2 & & \\
\hline 8104 & $6: 00$ & 16.833 & 159.5 & 149.3 & 147.2 & 150.8 & & & 145.7 & 133.1 & 140.5 & 128.2 & 117.8 & 119.8 & 136.6 & 128.0 & 123.3 & 159.9 & & \\
\hline 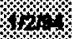 & 8.00 & 16.917 & 158.6 & 148.5 & 148.1 & 150.0 & & & 144.9 & 131.3 & 139.8 & 128.2 & 116.1 & 118.7 & 136.6 & 124.2 & 123.3 & 158.9 & & \\
\hline 1818 & $10: 00$ & 17.000 & 155.9 & 138.0 & 149.8 & 150.8 & & & 140.4 & 118.8 & 128.3 & 124.6 & 116.0 & 121.2 & 133.0 & 125.8 & 122.3 & 153.6 & & \\
\hline $8 \%$ & $12: 00$ & 17.083 & 157.6 & 135.3 & 152.3 & 150.7 & & & 136.8 & 120.6 & 130.0 & 118.7 & 118.4 & 123.8 & 129.4 & 128.2 & 128.2 & 146.5 & & \\
\hline 100 & $14: 00$ & 17.167 & 165.3 & 137.8 & 153.9 & 154.1 & & & 145.4 & 138.4 & 137.7 & 120.1 & 124.4 & 125.5 & 134.4 & 131.7 & 129.8 & 148.9 & & \\
\hline $31 \%$ & $18: 00$ & 17.250 & 168.7 & 127.4 & 153.8 & 156.8 & & & 155.7 & 153.1 & 149.8 & 133.2 & 125.2 & 126.3 & 141.2 & 132.4 & 129.7 & 163.7 & & \\
\hline $10 \%$ & $18: 00$ & 17.333 & 166.2 & 138.7 & 152.3 & $155: 9$ & 141.6 & & 155.0 & 153.2 & 152.5 & 135.0 & 122.7 & 123.7 & 144.0 & 129.1 & 128.1 & 168.3 & & \\
\hline 104 & $20: 00$ & 17.417 & 161.1 & 142.3 & 149.8 & 154.3 & 142.6 & & 149.1 & 143.6 & 147.4 & 132.5 & 119.4 & 123.0 & 439.1 & 127.6 & 128.8 & 164.1 & & \\
\hline 8124 & $22: 00$ & 17.500 & 161.2 & 145.0 & 148.9 & 153.5 & 140.1 & & 148.3 & 141.0 & 144.8 & 132.5 & 117.8 & 122.1 & 139.2 & 127.7 & 125.9 & 181.7 & & \\
\hline $4 \%$ & 0,00 & 17.603 & 161.3 & 145.0 & 149.0 & 152.7 & $\because \quad$ & & 146.8 & 135.7 & 142.2 & 129.9 & 117.9 & 121.3 & 138.4 & 127.8 & 125.8 & 161.7 & & \\
\hline$\%$ & $2: 00$ & 17.667 & 160.4 & 145.1 & 149.0 & 153.6 & & & 146.7 & 134.8 & 141.4 & 130.0 & 117.9 & 120.5 & 137.8 & 128.8 & 125.1 & 160.0 & & \\
\hline$\%$ & $4: 00$ & 17.760 & 161.3 & 145.1 & 148.2 & 153.6 & & & 145.8 & 134.9 & 142.3 & 130.9 & 117.8 & 123.1 & 139.3 & 127.0 & 125.1 & 160.1 & & \\
\hline$\%$ & $6: 00$ & 17.833 & 160.4 & 146.0 & 140.1 & 152.7 & & & 146.7 & 134.0 & 140.5 & 130.0 & 117.8 & 121.3 & 137.6 & 128.1 & 125.1 & 159.2 & & \\
\hline $2141 \%$ & $8: 00$ & 17.917 & 159.6 & 144.2 & 148.2 & 152.8 & & & 145.8 & 131.4 & 140.8 & 128.3 & 117.1 & 121.4 & 137.6 & 127.9 & 924.3 & 159.2 & & \\
\hline 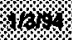 & $10: 00$ & 18.000 & 156.0 & 140.7 & 150.7 & 151.8 & & & 138.8 & 112.7 & 123.1 & 121.2 & 116.1 & 122.2 & 133.1 & 125.1 & 120.7 & 150.3 & & \\
\hline 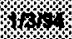 & $12: 00$ & 18.083 & 159.4 & 141.4 & 155.8 & 153.4 & & & 136.8 & 117.8 & 125.6 & 115.8 & 120.3 & 124.7 & 129.5 & 129.3 & 128.3 & 143.8 & & \\
\hline 818. & $14: 00$ & 18.167 & 167.0 & 149.1 & 157.4 & 152.3 & & & 147.1 & 138.1 & 136.8 & 122.7 & 124.4 & 128.1 & 135.3 & 134.3 & 130.6 & 148.8 & & \\
\hline $813 \%$ & $18: 00$ & 18.250 & 171.2 & & 159.0 & 152.2 & & & 157.3 & 156.6 & 150.6 & 133.1 & 128.6 & 129.7 & 142.0 & 134.1 & 133.0 & 160.9 & & \\
\hline$\%$ & $18: 00$ & 18.333 & 169.8 & & 154.8 & 150.6 & 132.8 & & 181.0 & 160.2 & 157.6 & 138.4 & 128.1 & 127.2 & 144.8 & 133.4 & 131.5 & 170.8 & & \\
\hline 14 & $20: 00$ & 88.417 & 168.3 & & 152.3 & & 137.3 & & 153.3 & 148.8 & 151.8 & 135.9 & 122.8 & 125.5 & 141.5 & 129.2 & 129.1 & 167.5 & & \\
\hline \% & $22: 00$ & 18.500 & 164.6 & & 152.4 & & 135.8 & & 151.7 & 144.4 & 147.3 & 133.3 & 122.9 & 126.4 & 141.6 & 131.0 & 129.1 & 164.1 & & \\
\hline א/14 & $0: 00$ & 18.583 & 164.7 & & 152.4 & & 139.2 & & 150.8 & 144.5 & 149.1 & 133.4 & 123.0 & 125.6 & 141.7 & 129.4 & 129.3 & 165.1 & & \\
\hline 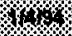 & $2: 00$ & 18.667 & 163.0 & & 152.5 & & 137.5 & & 151.8 & 141.0 & 145.7 & 133.4 & 120.4 & 124.8 & 141.0 & 130.4 & 127.6 & 162.8 & & \\
\hline $4 \%$ & $4: 00$ & 18.760 & 164.8 & & 152.5 & & 138.7 & & 151.0 & 140.1 & 145.7 & 134.3 & 121.3 & 124.8 & 141.9 & 129.5 & 128.5 & 165.3 & & \\
\hline 818 & $6: 00$ & 18.833 & 164.8 & & 150.8 & & 131.5 & & 150.2 & 137.5 & 145.7 & 132.6 & 122.2 & 124.8 & 141.0 & 130.4 & 127.7 & 162.7 & & \\
\hline $4 \%$ & $8: 00$ & 18.917 & 163.0 & & 151.7 & & & & 150.2 & 136.6 & 144.0 & 133.5 & 120.5 & 124.0 & 141.0 & 128.7 & 127.7 & 163.6 & & \\
\hline $3 \%$ & $10: 00$ & 49.000 & 157.7 & & 153.3 & & & & 140.5 & 109.2 & 124.0 & 122.8 & 118.7 & 125.6 & 134.8 & 127.7 & 123.2 & 150.2 & & \\
\hline \% & $12: 00$ & 19.093 & 159.3 & & 158.4 & & & & 137.7 & 117.8 & 124.7 & 117.6 & 122.8 & 128.2 & 132.0 & 130.1 & 129.1 & 143.9 & & \\
\hline$\%$ & $14: 00$ & 19.167 & 170.5 & & 160.9 & & & & 146.2 & 134.6 & 135.1 & 122.7 & 131.3 & 129.8 & 135.3 & 137.7 & 134.1 & 148.8 & & \\
\hline $4 \%$ & $16: 00$ & 19.250 & 174.8 & & 160.0 & 151.4 & 113.0 & & 168.1 & 162.8 & 156.7 & 137.5 & 130.4 & 132.4 & 148.2 & 135.1 & 135.7 & 168.0 & & \\
\hline $10 \%$ & $18: 00$ & 19.333 & 173.1 & & 155.7 & & 126.9 & & 185.4 & 168.2 & 162.8 & 141.9 & 127.8 & 129.8 & 148.3 & 133.5 & 132.4 & 171.7 & & \\
\hline$\%$ & $20: 00$ & 19.417 & 167.2 & & 153.3 & & 128.8 & & 156.0 & 155.0 & 154.3 & 137.7 & 124.0 & 127.3 & 145.1 & 131.0 & 130.0 & 168.5 & & \\
\hline $1 \%$ & $22: 00$ & 19.500 & 185.5 & & 153.3 & & 127.8 & & 152.6 & 148.0 & 150.8 & 136.9 & 124.7 & 128.5 & 142.6 & 131.1 & 129.2 & 168.8 & & \\
\hline 济 & 0.00 & 10.583 & 166.4 & & 153.3 & & 126.2 & & 152.7 & 144.5 & 149.1 & 135.1 & 123.0 & 126.5 & 141.8 & 131.2 & 129.3 & 165.1 & & \\
\hline $1 / 1$ & $2: 00$ & 19.667 & 164.7 & & 153.4 & & 121.8 & & 150.8 & 138.2 & 144.8 & 133.4 & 121.3 & 125.6 & 140.9 & 131.2 & 128.5 & 163.4 & & \\
\hline 1) & $4: 00$ & 19.750 & 164.7 & & 154.2 & & 118.5 & & 149.2 & 137.4 & 143.1 & 132.5 & 123.0 & 128.5 & 139.2 & 131.2 & 128.4 & 160.8 & & \\
\hline$\%$ & $6: 00$ & 10.833 & 164.7 & & 154.2 & & 115.8 & & 149.2 & 137.4 & 143.1 & 131.6 & 123.0 & 128.5 & 140.9 & 131.2 & 129.3 & 160.8 & & \\
\hline
\end{tabular}


Table 5. Tensiometer Data

\begin{tabular}{|c|c|c|c|c|c|c|c|c|c|c|c|c|c|c|c|c|c|c|c|c|}
\hline & & & & & & & & & Ten & Iometer & Data & & & & & & & & & \\
\hline & & Test & $\begin{array}{c}\text { Soll } \\
\text { Molsture } \\
\text { Tension } \\
\left(\mathrm{Cm} \mathrm{H}_{2} \mathrm{O}\right)\end{array}$ & $\begin{array}{c}\text { Soll } \\
\text { Moisture } \\
\text { Tension } \\
\text { (cm } \mathrm{H}_{2} \mathrm{O} \text { ) }\end{array}$ & $\begin{array}{c}\text { Soll } \\
\text { Molsture } \\
\text { Tension } \\
\left(\mathrm{Cm} \mathrm{H}_{2} \mathrm{O}\right)\end{array}$ & $\begin{array}{c}\text { Soll } \\
\text { Moisture } \\
\text { Tonsion } \\
\left(\mathrm{Cm} \mathrm{H}_{2} \mathrm{O}\right)\end{array}$ & $\begin{array}{c}\text { Soll } \\
\text { Molsture } \\
\text { Tenslon } \\
\left(\mathrm{Cm} \mathrm{H} \mathrm{H}_{2} \mathrm{O}\right)\end{array}$ & $\begin{array}{l}\text { Soll } \\
\text { Moisture } \\
\text { Tension } \\
\left(\mathrm{Cm} \mathrm{H}_{2} \mathrm{O}\right)\end{array}$ & $\begin{array}{c}\text { Soll } \\
\text { Molsture } \\
\text { Tension } \\
\left(\mathrm{Cm} \mathrm{H}_{2} \mathrm{O}\right) \\
\end{array}$ & 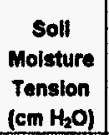 & $\begin{array}{c}\text { Soll } \\
\text { Molsture } \\
\text { Tension } \\
\left.\text { (cm } \mathrm{H}_{2} \mathrm{O}\right) \\
\end{array}$ & $\begin{array}{c}\text { soll } \\
\text { Molsture } \\
\text { Tension } \\
\left(\mathrm{Cm} \mathrm{H}_{2} \mathrm{O}\right)\end{array}$ & $\begin{array}{c}\text { Soll } \\
\text { Molsture } \\
\text { Tension } \\
\left.\text { (Cm } \mathrm{H}_{2} \mathrm{O}\right)\end{array}$ & $\begin{array}{c}\text { Soll } \\
\text { Molsture } \\
\text { Tension } \\
\left(\mathrm{Cm} \mathrm{H}_{2} \mathrm{O}\right)\end{array}$ & $\begin{array}{l}\text { Soll } \\
\text { Molisture } \\
\text { Tension } \\
\left.\text { (cm } \mathrm{H}_{2} \mathrm{O}\right)\end{array}$ & $\begin{array}{c}\text { Soll } \\
\text { Molsture } \\
\text { Tension } \\
\left(\mathrm{Cm} \mathrm{H}_{2} \mathrm{O}\right)\end{array}$ & $\begin{array}{c}\text { Soll } \\
\text { Molsture } \\
\text { Tension } \\
\left(\mathrm{em} \mathrm{H}_{2} \mathrm{O}\right)\end{array}$ & $\begin{array}{c}\text { Soll } \\
\text { Moisture } \\
\text { Tension } \\
\left(\mathrm{C} \mathrm{H}_{2} \mathrm{O}\right)\end{array}$ & $\begin{array}{c}\text { Soll } \\
\text { Molsture } \\
\text { Tension } \\
\left(\mathrm{cm} \mathrm{H}_{2} \mathrm{O}\right)\end{array}$ & 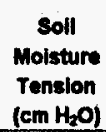 \\
\hline Date & Time & Days' & $\mathrm{C1}^{\circ}$ & $B 1^{*}$ & $A 1^{*}$ & $C 2^{\mathrm{b}}$ & $\mathbf{B 2}^{\mathbf{b}}$ & $A 2^{b}$ & $c 3^{c}$ & $\mathrm{B3}^{\mathrm{C}}$ & $\mathrm{A3}^{\mathrm{C}}$ & $C 4^{d}$ & $B 4^{d}$ & $A 4^{d}$ & $C 5^{\circ}$ & $B 5^{\circ}$ & $A 5^{\circ}$ & $\mathrm{cos}^{\mathrm{I}}$ & B6 ${ }^{1}$ & $A 6^{\prime}$ \\
\hline (1) & $8: 00$ & 19.917 & 164.7 & & 154.2 & & 115.0 & & 151.8 & 138.3 & 143.8 & 130.8 & 123.9 & 127.4 & 140.8 & 132.1 & 130.2 & 162.5 & & \\
\hline $1 \%$ & $10: 00$ & 20.000 & 162.8 & & 155.1 & & 108.1 & & 146.6 & 130.4 & 139.6 & 128.1 & 123.0 & 127.4 & 138.3 & 130.3 & 128.4 & 157.2 & & \\
\hline $8 \%$ & $12: 00$ & 20.083 & 152.4 & & 155.8 & & 84.7 & & 132.6 & 104.7 & 118.7 & 118.7 & 116.8 & 127.3 & 130.3 & 124.0 & 125.7 & 143.9 & & \\
\hline 84 & $14: 00$ & 20.167 & 173.9 & & 160.9 & 145.3 & 97.5 & & 158.3 & 145.2 & 140.2 & 1333.2 & 131.3 & 132.4 & 139.6 & 137.7 & 134.0 & 156.8 & & \\
\hline $81 \%$ & $16: 00$ & 20.250 & 178.5 & & 162.6 & 147.9 & 100.9 & & 157.4 & 150.4 & 149.7 & 134.0 & 131.2 & 134.1 & 142.9 & 142.0 & 137.4 & 161.0 & & \\
\hline \%or & $18: 00$ & 20.333 & 174.8 & & 158.3 & 147.8 & 110.4 & & 162.7 & 160.2 & 160.2 & 140.1 & 131.3 & 131.5 & 148.2 & 138.8 & 134.8 & 171.6 & & \\
\hline \%\$ & $20: 00$ & 20.417 & 169.7 & & 157.5 & & 114.8 & & 158.5 & 154.1 & 156.8 & 140.2 & 127.9 & 130.7 & 146.6 & 134.4 & 133.3 & 170.0 & & \\
\hline 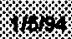 & $22: 00$ & 20.500 & 169.8 & & 156.7 & & 118.4 & & 157.7 & 150.8 & 155.1 & 140.3 & 127.2 & 129.8 & 145.9 & 133.6 & 132.8 & 171.1 & & \\
\hline 1104 & 0.00 & 20.683 & 168.1 & & 155.9 & & 118.4 & & 156.0 & 147.1 & 150.8 & 138.8 & 128.4 & 128.1 & 144.2 & 134.6 & 130.8 & 168.5 & & \\
\hline 46 & $2: 00$ & 20.667 & 168.1 & & 156.8 & & 118.5 & & 156.1 & 145.3 & 150.8 & 139.5 & 126.4 & 130.0 & 145.2 & 133.8 & 132.7 & 168.9 & & \\
\hline $1 \%$ & $4: 00$ & 20.750 & 168.2 & & 158.0 & & 120.2 & & 154.4 & 141.8 & 149.1 & 136.8 & 124.8 & 130.0 & 144.4 & 132.1 & 131.8 & 168.9 & & \\
\hline 8161 & $8: 00$ & 20.833 & 168.2 & & 158.0 & & 122.0 & & 156.2 & 143.6 & 148.1 & 138.6 & 126.5 & 130.0 & 145.3 & 134.7 & 132.8 & 169.8 & & \\
\hline $1,1 \%$ & $8: 00$ & 20.917 & 169.1 & & 158.0 & & 122.0 & & 154.4 & 141.8 & 149.1 & 137.8 & 128.5 & 130.0 & 145.3 & 134.7 & 132.8 & 167.0 & & \\
\hline 18.9 & $10: 00$ & 21.000 & 165.6 & & 154.2 & & 117.7 & & 154.4 & 137.5 & 142.2 & 135.2 & 124.8 & 128.3 & 143.5 & 126.9 & 130.2 & 184.3 & & \\
\hline \% & $12: 00$ & 21.083 & 160.3 & & 159.4 & & 108.1 & & 145.7 & 123.3 & 133.5 & 125.5 & 125.6 & 130.8 & 138.3 & 134.7 & 133.6 & 153.7 & & \\
\hline r. & $14: 00$ & 21.167 & 171.5 & & 162.0 & & 111.5 & & 151.7 & 142.7 & 142.1 & 132.4 & 131.6 & 133.5 & 141.6 & 140.6 & 138.9 & 161.5 & & \\
\hline $1016 \%$ & $18: 00$ & 21.250 & 178.4 & & 181.9 & & 120.1 & & 164.8 & 157.6 & 156.8 & 142.0 & 133.2 & 135.2 & 151.0 & 141.4 & 140.3 & 171.0 & & \\
\hline 164 & $18: 00$ & 21.333 & 175.9 & & 158.5 & & 130.5 & & 168.2 & 163.9 & 164.7 & 148.2 & 131.6 & 132.6 & 152.9 & 138.1 & 137.0 & 178.2 & & \\
\hline \% & $20: 00$ & 21.417 & 174.7 & & 156.8 & & 134.1 & & 163.1 & 157.8 & 163.1 & 145.7 & 128.3 & 131.8 & 150.5 & 136.5 & 1355.4 & 175.8 & & \\
\hline \% & $22: 00$ & 21.500 & 169.2 & & 156.1 & & 138.5 & & 158.9 & 151.7 & 158.8 & 144.8 & 128.6 & 131.9 & 148.9 & 136.6 & 133.8 & 175.0 & & \\
\hline 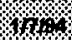 & $0: 00$ & 21.683 & 170.1 & & 155.2 & & 141.8 & & 158.0 & 149.9 & 158.2 & 142.2 & 127.5 & 131.0 & 148.0 & 136.7 & 134.7 & 172.4 & & \\
\hline $18=$ & $2: 00$ & 21.667 & 171.0 & & 155.3 & & 143.7 & & 157.2 & 147.3 & 155.3 & 142.3 & 128.7 & 130.2 & 148.1 & 135.8 & 133.8 & 172.5 & & \\
\hline $10 \%$ & $4: 00$ & 21.750 & 170.1 & & 158.1 & & 148.8 & & 157.2 & 147.3 & 154.5 & 142.3 & 126.7 & 131.0 & 148.1 & 135.0 & 133.0 & 171.6 & & \\
\hline $17 \%$ & $6: 00$ & 21.833 & 170.1 & & 155.3 & & 148.1 & & 157.3 & 147.3 & 154.5 & 142.3 & 128.8 & 131.4 & 148.1 & 135.0 & 133.9 & 170.8 & & \\
\hline $4 \%$ & $8: 00$ & 21.917 & 169.3 & & 155.3 & & 144.6 & & 155.5 & 143.8 & 151.9 & 142.3 & 125.8 & 130.2 & 149.0 & 135.0 & 133.8 & 170.8 & & \\
\hline 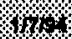 & $10: 00$ & 22.000 & 163.1 & & 157.8 & & & & 148.5 & 120.7 & 133.6 & 131.8 & 124.8 & 131.0 & 144.6 & 129.7 & 128.6 & 161.8 & & \\
\hline 87.4 & $12: 00$ & 22.083 & 161.3 & & 161.2 & & & & 141.4 & 113.6 & 129.2 & 121.2 & 127.4 & 132.7 & 138.4 & 135.6 & 135.4 & 153.0 & & \\
\hline 874 & $14: 00$ & 22.167 & 171.6 & & 162.9 & & & & 150.8 & 133.0 & 138.7 & 128.1 & 132.5 & 134.4 & 143.4 & 141.6 & 137.8 & 158.0 & & \\
\hline 87,4 & $16: 00$ & 22.250 & 181.8 & & 186.2 & & & 126.7 & 166.2 & 165.5 & 160.3 & 142.8 & 139.2 & 143.0 & 151.8 & 146.6 & 142.8 & 172.6 & & \\
\hline (1) & $18: 00$ & 22.333 & 180.2 & & 161.1 & & & 158.4 & 171.6 & 171.8 & 169.0 & 149.0 & 135.0 & 135.2 & 155.4 & 139.7 & 139.5 & 180.7 & & \\
\hline $8 \%$ & $20: 00$ & 22.417 & 175.2 & & 159.5 & & 144.4 & 102.4 & 164.9 & 164.9 & 167.4 & 146.5 & 130.0 & 132.7 & 152.2 & 138.3 & 136.3 & 177.5 & & \\
\hline 10.4 & 22:00 & 22.500 & 172.7 & & 157.0 & & 148.0 & $\begin{array}{l}98.9 \\
\end{array}$ & 180.7 & 156.1 & 182.3 & 145.7 & 129.3 & 132.8 & 152.4 & 138.8 & 135.8 & 175.1 & & \\
\hline 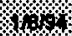 & $0: 00$ & 22.683 & 171.8 & & 157.9 & & 150.6 & 97.2 & 158.1 & 150.8 & 157.1 & 142.3 & 128.5 & 131.8 & 148.1 & 136.7 & 135.6 & 172.5 & & \\
\hline $4 \%$ & $2: 00$ & 22.667 & 171.0 & & 156.2 & & 148.1 & 95.5 & 157.3 & 148.2 & 156.2 & 142.3 & 125.8 & 131.8 & 148.1 & 135.0 & 134.8 & 170.8 & & \\
\hline $8 \%$ & $4: 00$ & 22.750 & 170.1 & 151.3 & 157.0 & & 147.2 & 93.7 & 157.3 & 143.8 & 153.6 & 139.7 & 128.8 & 131.8 & 148.1 & 136.8 & 133.9 & 169.0 & & \\
\hline 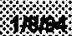 & $6: 00$ & 22.633 & 171.9 & 151.3 & 157.0 & & 144.6 & 93.7 & 158.1 & 147.3 & 154.5 & 142.3 & 128.8 & 131.0 & 148.1 & 137.6 & 134.8 & 171.7 & & \\
\hline $8 / 40$ & $8: 00$ & 22.917 & 171.0 & 154.8 & 157.8 & & 139.4 & 90.2 & 157.3 & 143.8 & 152.8 & 142.3 & 125.9 & 132.0 & 148.2 & 135.0 & 134.0 & 170.8 & & \\
\hline $8170 \%$ & $0: 00$ & 23.000 & 161.3 & 151.2 & 156.1 & & & & 144.1 & 112.8 & 124.9 & 128.5 & 125.8 & 131.8 & 140.2 & 131.4 & 129.5 & 158.4 & & \\
\hline
\end{tabular}


Table 5. Tensiometer Data

\begin{tabular}{|c|c|c|c|c|c|c|c|c|c|c|c|c|c|c|c|c|c|c|c|c|}
\hline & & & & & & & & & Ten & siometer & Data & & & & & & & & & \\
\hline & & Test & $\begin{array}{c}\text { Soll } \\
\text { Molsture } \\
\text { Tension } \\
\left(\mathrm{Cm} \mathrm{H}_{2} \mathrm{O}\right)\end{array}$ & $\begin{array}{c}\text { Soll } \\
\text { Molsture } \\
\text { Tenslon } \\
\left(\mathrm{Cm} \mathrm{H}_{2} \mathrm{O}\right) \\
\end{array}$ & $\begin{array}{c}\text { Soll } \\
\text { Molsture } \\
\text { Tension } \\
\left(\mathrm{Cm} \mathrm{H}_{2} \mathrm{O}\right)\end{array}$ & 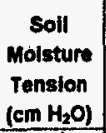 & $\begin{array}{c}\text { Soil } \\
\text { Moisture } \\
\text { Tension } \\
\left(\mathrm{Cm}^{2} \mathrm{H}_{2} \mathrm{O}\right) \\
\end{array}$ & $\begin{array}{c}\text { Soll } \\
\text { Moisture } \\
\text { Tension } \\
\left(\mathrm{Cm} \mathrm{H}_{2} \mathrm{O}\right)\end{array}$ & $\begin{array}{c}\text { Soll } \\
\text { Molsture } \\
\text { Tension } \\
\left(\mathrm{em} \mathrm{H}_{2} \mathrm{O}\right)\end{array}$ & $\begin{array}{c}\text { Soll } \\
\text { Moisture } \\
\text { Tension } \\
\left(\mathrm{Cm} \mathrm{H}_{2} \mathrm{O}\right)\end{array}$ & $\begin{array}{c}\text { Solf } \\
\text { Molsture } \\
\text { Tonsion } \\
\left.\text { (cm } \mathrm{H}_{2} \mathrm{O}\right) \\
\end{array}$ & $\begin{array}{c}\text { Soil } \\
\text { Molsture } \\
\text { Tension } \\
\left(\mathrm{cm}_{2} \mathrm{O}\right) \\
\end{array}$ & $\begin{array}{c}\text { Soll } \\
\text { Moisture } \\
\text { Tension } \\
\left(\mathrm{Cm} \mathrm{H}_{2} \mathrm{O}\right)\end{array}$ & $\begin{array}{c}\text { Soll } \\
\text { Molsture } \\
\text { Tension } \\
\text { (cm } \mathrm{H}_{2} \mathrm{O} \text { ) }\end{array}$ & $\begin{array}{c}\text { Soll } \\
\text { Moisture } \\
\text { Tension } \\
\left(\mathrm{Cm} \mathrm{H}_{2} \mathrm{O}\right)\end{array}$ & $\begin{array}{c}\text { Soll } \\
\text { Molsture } \\
\text { Tenslon } \\
\left(\mathrm{Cm} \mathrm{H}_{2} \mathrm{O}\right)\end{array}$ & $\begin{array}{c}\text { Soll } \\
\text { Molsture } \\
\text { Tension } \\
\left(\mathrm{Cm} \mathrm{H}_{2} \mathrm{O}\right)\end{array}$ & $\begin{array}{c}\text { soll } \\
\text { Moisture } \\
\text { Tansion } \\
\left(\mathrm{Cm} \mathrm{H}_{2} \mathrm{O}\right)\end{array}$ & $\begin{array}{c}\text { soll } \\
\text { Molsture } \\
\text { Tension } \\
\left(\mathrm{Cm} \mathrm{H} \mathrm{H}_{2} \mathrm{O}\right)\end{array}$ & $\begin{array}{c}\text { Soll } \\
\text { Molsture } \\
\text { Tenstion } \\
\left(\mathrm{Cm} \mathrm{H}_{2} \mathrm{O}\right) \\
\end{array}$ \\
\hline Date & Time & Days' & $C 1^{\circ}$ & $B 1^{*}$ & $A 1^{*}$ & $\mathrm{C2}^{\circ}$ & $B 2^{6}$ & $A 2^{b}$ & $\mathrm{C}^{\mathrm{C}}$ & $83^{\mathrm{C}}$ & $\mathrm{A3}^{\mathrm{C}}$ & $\mathrm{CA}^{\mathrm{d}}$ & $B 4^{d}$ & $\mathrm{~A}^{\mathrm{d}}$ & $C 5^{\circ}$ & $B 5^{\circ}$ & $A 5^{\circ}$ & $c 6^{\prime}$ & $\mathrm{BG}^{\prime}$ & $A 6^{\prime}$ \\
\hline$\%$ wo & \begin{tabular}{|l|}
$12: 00$ \\
\end{tabular} & 23.083 & 161.2 & & 166.4 & & & & 139.8 & 108.3 & 124.0 & 120.3 & 128.2 & 134.4 & 138.3 & 134.7 & 134.5 & 150.3 & & \\
\hline 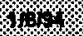 & 14:00 & 23.167 & 971.5 & & 167.1 & & & 80.8 & 148.8 & 132.8 & 138.6 & 128.0 & 135.0 & 135.2 & 143.2 & 140.5 & 139.4 & 157.9 & & \\
\hline \% & $16: 00$ & 23.260 & 185.2 & & 167.8 & & & 98.7 & 160.5 & 166.3 & 159.3 & 145.3 & 139.1 & 140.3 & 154.2 & 147.3 & 144.3 & 173.3 & & \\
\hline \% & \begin{tabular}{|l|}
$18: 00$ \\
\end{tabular} & 23.333 & 181.9 & & 162.8 & & & 93.5 & 173.3 & 177.1 & 169.8 & 150.7 & 135.9 & 136.8 & 158.2 & 141.5 & 140.3 & 181.5 & & \\
\hline 14: & $20: 00$ & 23.417 & 176.0 & & 161.2 & & 132.3 & & 164.8 & 164.8 & 164.7 & 147.3 & 130.8 & 134.4 & 152.1 & 137.3 & 137.1 & 177.4 & & \\
\hline 144 & 22:00 & 23.600 & 175.2 & $\cdots$ & 161.2 & & 132.3 & & 161.4 & 158.7 & 159.8 & 143.9 & 130.0 & 134.4 & 149.8 & 138.2 & 137.2 & 172.2 & & \\
\hline א/19\% & $0: 00$ & 23.683 & 175.2 & & 160.4 & & 130.8 & & 161.4 & 155.1 & 157.8 & 143.0 & 130.0 & 134.4 & 149.6 & 138.3 & 137.2 & 174.0 & & \\
\hline $6 \%$ & $2: 00$ & 23.667 & 175.2 & & 180.4 & & 128.1 & & 161.5 & 154.3 & 159.6 & 144.8 & 130.1 & 134.5 & 150.6 & 138.3 & 137.2 & 172.3 & & \\
\hline K17. & $4: 00$ & 23.760 & 175.3 & & 159.6 & & 130.7 & & 161.5 & 151.7 & 156.2 & 142.2 & 130.1 & 134.5 & 148.9 & 138.4 & 136.4 & 172.4 & & \\
\hline W10\% & $8: 00$ & 23.833 & 173.5 & & 161.4 & & & & 160.7 & 151.7 & 158.2 & 142.2 & 130.2 & 133.6 & 148.9 & 137.5 & 138.5 & 173.3 & & \\
\hline $48 \%$ & $8: 00$ & 23.917 & 172.7 & & 158.7 & & & & 159.8 & 146.4 & 153.6 & 142.3 & 128.4 & 135.4 & 149.8 & 135.8 & 135.6 & 171.6 & & \\
\hline 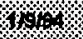 & \begin{tabular}{|l|}
$10: 00$ \\
\end{tabular} & 24.000 & 164.8 & & 159.5 & & & & 145.8 & 116.3 & 126.6 & 128.2 & 125.7 & 133.6 & 141.9 & 132.2 & 131.1 & 158.3 & & \\
\hline$\%$ & $12: 00$ & 24.083 & 162.9 & & 168.2 & & & & 141.4 & 110.9 & 125.7 & 121.1 & 131.7 & 137.0 & 138.3 & 138.1 & 137.0 & 151.1 & & \\
\hline 14. & 14:00 & 24.167 & 171.5 & & 168.0 & & & & 147.3 & 128.7 & 135.1 & 126.3 & 134.1 & 136.8 & 144.1 & 139.7 & 139.4 & 156.1 & & \\
\hline WHy & $16: 00$ & 24.250 & 186.0 & & 170.4 & & & & 168.7 & 161.0 & 156.7 & 143.6 & 142.5 & 143.8 & 155.0 & 148.1 & 144.3 & 172.4 & & \\
\hline $16 \%$ & 18:00 & 24.333 & 188.7 & & 168.2 & & & & 180.0 & 180.5 & 173.2 & 155.8 & 139.2 & 141.2 & 161.2 & 145.6 & 145.3 & 183.8 & & \\
\hline 1010) & $20: 00$ & 24.417 & 182.0 & & 163.8 & & & & 173.4 & 175.3 & 172.5 & 154.3 & 135.1 & 137.8 & 159.0 & 141.6 & 141.3 & 181.7 & & \\
\hline 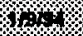 & $22: 00$ & 24.500 & 178.6 & & 162.1 & & 127.1 & & 168.3 & 186.8 & 169.1 & 150.0 & 132.6 & 137.0 & 154.8 & 141.7 & 139.7 & 179.2 & & \\
\hline 13. & $0: 00$ & 24.583 & 178.7 & & 162.1 & & 130.6 & & 168.6 & 164.9 & 167.4 & 149.1 & 133.5 & 138.2 & 154.0 & 140.8 & 139.8 & 176.7 & & \\
\hline 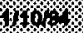 & $2: 00$ & 24.667 & 176.1 & & 162.2 & & 131.6 & & 168.7 & 158.7 & 164.0 & 947.5 & 133.6 & 138.0 & 154.1 & 141.0 & 139.0 & 175.9 & & \\
\hline *is & $4: 00$ & 24.750 & 176.2 & & 161.3 & & 130.7 & & 164.1 & 157.0 & 162.3 & 145.7 & 131.8 & 136.3 & 153.2 & 141.0 & 139.0 & 175.1 & & \\
\hline \%1, & $6: 00$ & 24.833 & 176.2 & & 162.2 & & & & 163.3 & 152.6 & 158.7 & 147.5 & 131.9 & 138.0 & 152.4 & 140.2 & 139.0 & 175.1 & & \\
\hline 3104 & $8: 00$ & 24.917 & 175.3 & & 162.2 & & 125.5 & & 161.5 & 150.8 & 157.8 & 145.7 & 130.2 & 136.3 & 152.4 & 139.3 & 138.2 & 174.2 & & \\
\hline $110 \%$ & $10: 00$ & 25.000 & 166.5 & & 161.3 & & 113.4 & & 151.0 & 119.8 & 129.2 & 131.7 & 128.3 & 135.3 & 145.3 & 134.8 & 132.9 & 161.8 & & \\
\hline $10 \%$ & 12:00 & 25.083 & 164.8 & & 168.1 & & 104.6 & & 144.8 & 112.7 & 128.3 & 124.6 & 132.5 & 138.7 & 141.7 & 138.1 & 139.6 & 157.2 & & \\
\hline 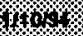 & \begin{tabular}{|l|}
$14: 00$ \\
\end{tabular} & 25.167 & 175.8 & & 171.5 & & 104.5 & & 152.4 & 130.2 & 139.4 & 132.4 & 140.1 & 140.4 & 147.5 & 147.5 & 144.5 & 161.3 & & \\
\hline 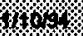 & \begin{tabular}{|l|}
$16: 00$ \\
\end{tabular} & 25.250 & 191.3 & & 173.9 & & 114.7 & & 175.6 & 166.3 & 162.8 & 148.8 & 146.0 & 145.5 & 158.5 & 155.1 & 149.5 & 177.7 & & \\
\hline 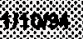 & \begin{tabular}{|l|}
$18: 00$ \\
\end{tabular} & 26.333 & 193.1 & & 168.8 & & 126.9 & & 185.3 & 188.4 & 180.2 & 182.0 & 143.6 & 142.1 & 164.8 & 149.2 & 148.8 & 189.3 & & \\
\hline 11014 & $20: 00$ & 25.417 & 184.7 & & 164.7 & & 134.0 & & 178.7 & 182.5 & 180.4 & 159.6 & 138.7 & 138.8 & 161.7 & 144.3 & 143.1 & 188.0 & & \\
\hline 140.4 & $22: 00$ & 25.600 & 180.4 & & 163.0 & & 139.3 & & 171.8 & 173.7 & 175.2 & 156.2 & 137.0 & 138.8 & 158.3 & 142.7 & 142.4 & 182.8 & & \\
\hline (4) & $0: 00$ & 25.683 & 178.6 & & 163.1 & & 141.1 & & 168.4 & 168.5 & 174.8 & 152.7 & 136.2 & 138.8 & 157.5 & 142.7 & 140.7 & 181.2 & & \\
\hline 4 & $2: 00$ & 25.667 & 179.7 & & 163.1 & & 143.7 & & 168.5 & 165.8 & 170.1 & 152.8 & 135.4 & 138.9 & 157.6 & 141.9 & 139.9 & 179.5 & & \\
\hline 1/1/\% & $4: 00$ & 25.750 & 178.8 & & 184.0 & & 144.6 & & 166.8 & 183.2 & 168.4 & 151.0 & 134.6 & 139.0 & 155.8 & 142.0 & 140.9 & 179.6 & & \\
\hline 41414 & $6: 00$ & 25.833 & 178.8 & & 163.2 & & 142.9 & & 165.1 & 158.8 & 164.9 & 150.2 & 134.6 & 138.1 & 155.1 & 142.0 & 140.8 & 177.8 & & \\
\hline (1) & $8: 00$ & 25.017 & 176.2 & & 161.4 & & 136.0 & & 163.4 & 150.9 & 159.7 & 147.5 & 132.0 & 138.1 & 153.3 & 130.4 & 139.1 & 175.2 & & \\
\hline (1) & \begin{tabular}{|l|}
$10: 00$ \\
\end{tabular} & 26.000 & 188.3 & & 148.1 & & 118.8 & 123.4 & 154.6 & 83.6 & 99.7 & 135.2 & 118.7 & 139.8 & 948.0 & 116.5 & 118.1 & 165.4 & & \\
\hline 414 & 12:00 & 26.083 & 161.2 & & 170.8 & & 108.1 & & 139.6 & 118.9 & 125.7 & 121.1 & 134.3 & 139.7 & 139.2 & 142.5 & 141.4 & 152.9 & & \\
\hline (2) & $14: 00$ & 26.167 & 176.7 & & 173.2 & & 108.2 & & 150.7 & 135.5 & 141.2 & 130.8 & 142.7 & 142.1 & 146.7 & 148.4 & 146.3 & 160.4 & & \\
\hline
\end{tabular}


Table 5. Tensiometer Data

\begin{tabular}{|c|c|c|c|c|c|c|c|c|c|c|c|c|c|c|c|c|c|c|c|c|}
\hline & & & & & & & & & Ten & siometer $\mathrm{C}$ & Data & & & & & & & & & \\
\hline & & Test & $\begin{array}{c}\text { Soll } \\
\text { Moisture } \\
\text { Tenslon } \\
\left(\mathrm{Cm} \mathrm{H}_{2} \mathrm{O}\right) \\
\end{array}$ & $\begin{array}{c}\text { Soll } \\
\text { Molsture } \\
\text { Tension } \\
\left(\mathrm{em} \mathrm{H}_{2} \mathrm{O}\right) \\
\end{array}$ & $\begin{array}{c}\text { Soll } \\
\text { Moisture } \\
\text { Tension } \\
\left(\mathrm{Cm} \mathrm{H}_{2} \mathrm{O}\right) \\
\end{array}$ & $\begin{array}{c}\text { Soil } \\
\text { Moisture } \\
\text { Tension } \\
\left(\mathrm{Cm}_{2} \mathrm{O}\right) \\
\end{array}$ & $\begin{array}{c}\text { Solf } \\
\text { Moisture } \\
\text { Tension } \\
\left(\mathrm{cm} \mathrm{H}_{2} \mathrm{O}\right) \\
\end{array}$ & 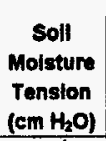 & $\begin{array}{c}\text { Soil } \\
\text { Molsture } \\
\text { Tension } \\
\left(\mathrm{Cm} \mathrm{H}_{2} \mathrm{O}\right) \\
\end{array}$ & $\begin{array}{c}\text { Soll } \\
\text { Moisture } \\
\text { Tansion } \\
\left(\mathrm{cm} \mathrm{H}_{2} \mathrm{O}\right)\end{array}$ & $\begin{array}{c}\text { Soll } \\
\text { Moisture } \\
\text { Tension } \\
\left(\mathrm{Cm} \mathrm{H}_{2} \mathrm{O}\right) \\
\end{array}$ & $\begin{array}{c}\text { Soll } \\
\text { Moisture } \\
\text { Tension } \\
\left(\mathrm{Cm} \mathrm{H}_{2} \mathrm{O}\right) \\
\end{array}$ & 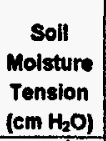 & $\begin{array}{c}\text { Soll } \\
\text { Molsture } \\
\text { Tension } \\
\left(\mathrm{Cm} \mathrm{H}_{2} \mathrm{O}\right) \\
\end{array}$ & $\begin{array}{c}\text { Soll } \\
\text { Moisture } \\
\text { Tension } \\
\left(\mathrm{cm} \mathrm{H} \mathrm{H}_{2} \mathrm{O}\right)\end{array}$ & 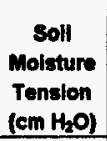 & $\begin{array}{c}\text { Soil } \\
\text { Moisture } \\
\text { Tension } \\
\left(\mathrm{cm} \mathrm{H}_{2} \mathrm{O}\right) \\
\end{array}$ & 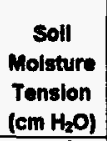 & $\begin{array}{c}\text { Soll } \\
\text { Molsture } \\
\text { Tonslon } \\
\left(\mathrm{cm} \mathrm{H}_{2} \mathrm{O}\right) \\
\end{array}$ & $\begin{array}{c}\text { Soll } \\
\text { Molsture } \\
\text { Tension } \\
\left(\mathrm{Cm} \mathrm{H}_{2} \mathrm{O}\right) \\
\end{array}$ \\
\hline Date & Time & Days ${ }^{1}$ & $\mathrm{C1}^{2}$ & $B 1^{\prime \prime}$ & $A 1^{\prime \prime}$ & $\mathrm{Cl}^{\mathrm{b}}$ & $\mathrm{B2}^{6}$ & $A 2^{b}$ & $c 3^{c}$ & $83^{c}$ & $A 3^{\circ}$ & $\mathrm{C}^{\mathrm{d}}$ & $B 4^{d}$ & $A 4^{d}$ & $\mathrm{Cs}^{\circ}$ & $B 5^{\circ}$ & $A 5^{\circ}$ & $C 6^{\prime}$ & $86^{\prime}$ & $A 6^{\prime}$ \\
\hline $64 \%$ & $16: 00$ & 26.250 & 190.4 & & 173.9 & & 114.7 & & 173.0 & 163.7 & 161.0 & 148.2 & 146.9 & 147.3 & 158.5 & 153.3 & 150.4 & 176.8 & & \\
\hline $14 \%$ & $18: 00$ & 26.333 & 194.0 & & 169.7 & & 126.9 & & 185.3 & 185.8 & 182.8 & 159.4 & 143.6 & 144.7 & 164.8 & 150.1 & 149.7 & 189.3 & & \\
\hline $140 \%$ & $20: 00$ & 26.417 & 185.6 & & 168.4 & & 132.3 & & 179.5 & 179.8 & 181.2 & 158.7 & 139.5 & 140.5 & 159.8 & 144.3 & 144.0 & 186.2 & & \\
\hline $1410 \%$ & $22: 00$ & 26.500 & 183.0 & & 185.7 & & 132.4 & & 173.6 & 172.8 & 178.1 & 155.3 & 137.0 & 140.6 & 158.3 & 143.5 & 142.4 & 182.8 & & \\
\hline 1404 & $0: 00$ & 26.683 & 180.5 & & 165.7 & & 130.7 & & 170.2 & 164.9 & 172.7 & 152.7 & 138.8 & 141.5 & 157.5 & 143.6 & 142.5 & 180.3 & & \\
\hline $14 \%$ & $2: 00$ & 26.667 & 179.7 & & 184.0 & & 129.9 & & 167.6 & 164.9 & 170.1 & 152.7 & 137.1 & 139.8 & 158.4 & 143.7 & 141.6 & 179.5 & & \\
\hline $1119 \%$ & $4: 00$ & 26.750 & 180.5 & & 164.9 & & 130.7 & & 168.5 & 161.4 & 167.5 & 152.8 & 137.2 & 139.8 & 156.8 & 143.7 & 142.5 & 180.4 & & \\
\hline $142 \% \%$ & $6: 00$ & 26.833 & 179.7 & & 165.8 & & 128.2 & & 166.8 & 158.8 & 168.8 & 151.0 & 137.2 & 140.7 & 158.8 & 142.8 & 143.4 & 177.8 & & \\
\hline 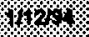 & $8: 00$ & 26.917 & 179.7 & & 164.0 & & 125.6 & & 166.8 & 154.4 & 163.2 & 150.2 & 134.6 & 139.8 & 156.8 & 142.0 & 140.0 & 178.7 & & \\
\hline $192 \%$ & $10: 00$ & 27.000 & 170.0 & & 164.8 & & 115.1 & & 154.5 & 130.4 & 137.0 & 140.5 & 133.6 & 138.9 & 150.8 & 137.5 & 137.2 & 167.1 & & \\
\hline 1429 & $12: 00$ & 27.083 & 165.5 & & 169.9 & & 106.4 & & 146.6 & 118.9 & 129.1 & 128.1 & 135.2 & 141.4 & 143.5 & 141.7 & 140.5 & 157.3 & & \\
\hline 16294 & $14: 00$ & 27.167 & 176.7 & & 173.2 & & 104.5 & & 151.6 & 130.3 & 138.6 & 133.3 & 143.8 & 143.0 & 149.3 & 148.4 & 146.3 & 162.2 & & \\
\hline $40 \%$ & 16:00 & 27.250 & 188.7 & & 174.8 & & 112.1 & & 170.4 & 155.7 & 156.7 & 145.3 & 147.8 & 149.0 & 159.4 & 155.1 & 151.3 & 176.8 & & \\
\hline $140 \%$ & 18:00 & 27.333 & 194.9 & & 172.3 & & 120.0 & & 185.3 & 178.7 & 176.7 & 159.4 & 147.0 & 145.6 & 168.5 & 152.7 & 150.6 & 189.2 & & \\
\hline 811291 & $20: 00$ & 27.417 & 189.0 & & 168.1 & & 127.1 & & 181.2 & 176.2 & 177.7 & 158.5 & 141.2 & 144.0 & 163.3 & 146.8 & 147.4 & 187.9 & & \\
\hline $412 \%$ & 22:00 & 27.500 & 183.9 & & 167.4 & & 132.3 & & 175.3 & 171.9 & 176.1 & 156.1 & 141.3 & 143.2 & 161.7 & 147.0 & 144.8 & 184.5 & & \\
\hline $44 \%$ & $0: 00$ & 27.583 & 183.1 & & 167.4 & & 132.4 & & 171.9 & 169.3 & 170.8 & 154.4 & 139.7 & 142.3 & 159.2 & 146.2 & 143.3 & 181.1 & & \\
\hline 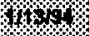 & 2:00 & 27.067 & 183.1 & & 165.7 & & 134.2 & & 171.9 & 164.9 & 169.2 & 153.6 & 139.7 & 141.5 & 158.4 & 147.1 & 145.1 & 181.2 & & \\
\hline $84 \%$ & $4: 00$ & 27.760 & 183.1 & & 168.6 & & 135.0 & & 170.2 & 163.2 & 170.1 & 152.7 & 139.7 & 141.5 & 159.3 & 145.4 & 144.2 & 179.5 & & \\
\hline 140 & $6: 00$ & 27.833 & 180.5 & & 186.6 & & 132.5 & & 169.4 & 161.4 & 167.5 & 152.7 & 138.9 & 142.4 & 159.4 & 145.4 & 144.3 & 179.5 & & \\
\hline $4140 \%$ & $8: 00$ & 27.917 & 179.7 & & 166.6 & & 130.8 & & 168.5 & 157.8 & 164.0 & 151.8 & 137.2 & 141.6 & 158.5 & 143.7 & 143.4 & 178.5 & & \\
\hline $1100 \%$ & 10:00 & 28.000 & 171.7 & & 967.4 & & 116.8 & & & 130.4 & 135.3 & 140.4 & 135.3 & 141.5 & 150.6 & 137.4 & 139.0 & 168.8 & & \\
\hline $14,1 \%$ & $12: 00$ & 28.083 & 164.6 & & 174.3 & & 106.4 & & & 118.8 & 127.4 & 127.2 & 137.7 & 143.1 & 144.3 & 143.3 & 143.1 & 158.1 & & \\
\hline $4_{14}$ & $14: 00$ & 28.167 & 178.8 & & 174.9 & & 104.5 & & & 130.2 & 138.5 & 131.5 & 145.3 & 143.9 & 149.2 & 150.8 & 148.0 & 161.2 & & \\
\hline$\sqrt{10} 18 \%$ & $16: 00$ & 28.250 & 193.8 & & 177.4 & & 113.0 & & 175.5 & 161.9 & 160.1 & 152.3 & 152.0 & 152.5 & 161.8 & 158.5 & 154.6 & 180.2 & & \\
\hline 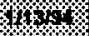 & 18:00 & 28.333 & 197.5 & & 174.1 & & 123.4 & & 188.7 & 182.3 & 180.2 & 162.0 & 150.5 & 148.2 & 168.2 & 156.1 & 154.0 & 191.0 & & \\
\hline $54 \% 4$ & $20: 00$ & 28.417 & 191.6 & & 170.7 & & 126.2 & & 181.2 & 179.8 & 181.2 & 160.4 & 144.7 & 145.7 & 165.9 & 151.2 & 149.1 & 187.8 & & \\
\hline 11414 & $22: 00$ & 28.500 & 187.3 & & 170.0 & & 134.1 & & 178.7 & 176.3 & 180.4 & 158.7 & 141.3 & 144.9 & 164.7 & 148.7 & 146.7 & 188.2 & & \\
\hline 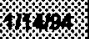 & $0: 00$ & 28.583 & 184.8 & & 189.1 & & 130.8 & & 175.3 & 172.8 & 176.9 & 157.0 & 140.5 & 145.0 & 161.8 & 147.9 & 146.7 & 184.6 & & \\
\hline $41 \%$ & $2: 00$ & 28.607 & 185.7 & & 168.3 & & 131.6 & & 175.4 & 172.0 & 178.1 & 156.2 & 140.6 & 145.0 & 161.8 & 147.1 & 145.8 & 182.9 & & \\
\hline $4 \%$ & $4: 00$ & 28.750 & 184.0 & & 169.2 & & 129.0 & & 172.0 & 167.6 & 172.7 & 155.4 & 140.6 & 145.0 & 159.3 & 147.2 & 146.0 & 183.0 & & \\
\hline 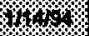 & 6:00 & 28.833 & 183.2 & & 168.4 & & 130.8 & & 171.1 & 165.0 & 170.1 & 155.4 & 140.6 & 145.1 & 160.2 & 147.2 & 146.0 & 181.3 & & \\
\hline $44 \%$ & $8: 00$ & 28.917 & 182.3 & & 187.5 & & 125.6 & & 170.3 & 160.5 & 167.5 & 152.8 & 138.8 & 144.2 & 159.4 & 145.5 & 143.4 & 181.3 & & \\
\hline 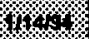 & $10: 00$ & 29.000 & 172.6 & & 188.3 & & 114.3 & & & 138.6 & 142.3 & 137.8 & 136.2 & 142.4 & 151.5 & 139.2 & 139.9 & 168.9 & & \\
\hline (1) & $12: 00$ & 29.083 & 164.7 & & 173.4 & & 101.2 & & & 115.3 & 123.9 & 123.7 & 138.6 & 144.0 & 145.2 & 141.6 & 142.2 & 157.2 & & \\
\hline 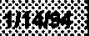 & $14: 00$ & 29.167 & 174.9 & & 175.0 & & 97.6 & & & 125.8 & 135.1 & 131.5 & 143.6 & 143.9 & 151.0 & 147.5 & 146.3 & 161.3 & & \\
\hline $41 \%$ & $16: 00$ & 29.260 & 195.6 & & 179.2 & & 107.0 & & 177.4 & 161.8 & 161.9 & 151.5 & 153.9 & 154.3 & 163.8 & 161.3 & 156.5 & 181.2 & & \\
\hline 1 & 18:00 & 29.333 & 197.5 & & 175.9 & & 116.6 & & 188.8 & 181.4 & 180.2 & 163.8 & 151.5 & 150.9 & 169.2 & 158.0 & 155.8 & 191.1 & & \\
\hline
\end{tabular}


Table 5. Tensiometer Data

\begin{tabular}{|c|c|c|c|c|c|c|c|c|c|c|c|c|c|c|c|c|c|c|c|c|}
\hline & & & & & & & & & Ten & siometer & Data & & & & & & & & & \\
\hline & & Test & $\begin{array}{c}\text { Soil } \\
\text { Moisture } \\
\text { Tension } \\
\left(\mathrm{Cm} \mathrm{H}_{2} \mathrm{O}\right)\end{array}$ & $\begin{array}{c}\text { Soll } \\
\text { Molsture } \\
\text { Tension } \\
\left(\mathrm{Cm} \mathrm{H} \mathrm{H}_{2} \mathrm{O}\right)\end{array}$ & $\begin{array}{c}\text { Soll } \\
\text { Molsture } \\
\text { Tenslon } \\
\left(\mathrm{cm} \mathrm{H}_{2} \mathrm{O}\right)\end{array}$ & $\begin{array}{c}\text { Soll } \\
\text { Moisture } \\
\text { Tension } \\
\left(\mathrm{Cm} \mathrm{H}_{2} \mathrm{O}\right)\end{array}$ & $\begin{array}{c}\text { Soil } \\
\text { Moisture } \\
\text { Tenslon } \\
\left(\mathrm{em} \mathrm{H}_{2} \mathrm{O}\right)\end{array}$ & $\begin{array}{c}\text { Soll } \\
\text { Molsture } \\
\text { Tension } \\
\left(\mathrm{Cm} \mathrm{H} \mathrm{H}_{2} \mathrm{O}\right)\end{array}$ & $\begin{array}{c}\text { Soll } \\
\text { Molsture } \\
\text { Tension } \\
\left(\mathrm{Cm} \mathrm{H}_{2} \mathrm{O}\right)\end{array}$ & $\begin{array}{c}\text { soll } \\
\text { Molsture } \\
\text { Tension } \\
\left(\mathrm{em}_{2} \mathrm{O}\right)\end{array}$ & $\begin{array}{c}\text { Soll } \\
\text { Molsture } \\
\text { Tension } \\
\left(\mathrm{Cm} \mathrm{H}_{2} \mathrm{O}\right) \\
\end{array}$ & 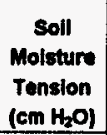 & $\begin{array}{c}\text { Soll } \\
\text { Molsture } \\
\text { Tension } \\
\left.\text { (em } \mathrm{H}_{2} \mathrm{O}\right)\end{array}$ & $\begin{array}{c}\text { Soll } \\
\text { Molature } \\
\text { Tenslon } \\
\left(\mathrm{Cm} \mathrm{H}_{2} \mathrm{O}\right) \\
\end{array}$ & $\begin{array}{c}\text { Soll } \\
\text { Molsture } \\
\text { Tension } \\
\left.\text { (em } \mathrm{H}_{2} \mathrm{O}\right)\end{array}$ & $\begin{array}{c}\text { Soll } \\
\text { Molasture } \\
\text { Tension } \\
\left(\mathrm{cm} \mathrm{H} \mathrm{H}_{2} \mathrm{O}\right)\end{array}$ & $\begin{array}{c}\text { Soll } \\
\text { Molsture } \\
\text { Tenslon } \\
\left(\mathrm{em} \mathrm{H}_{2} \mathrm{O}\right)\end{array}$ & $\begin{array}{c}\text { Soll } \\
\text { Molsture } \\
\text { Tension } \\
\left(\mathrm{Cm} \mathrm{H}_{2} \mathrm{O}\right) \\
\end{array}$ & $\begin{array}{c}\text { Soll } \\
\text { Moisture } \\
\text { Tension } \\
\left(\mathrm{cm} \mathrm{H} \mathrm{H}_{2} \text { ) }\right.\end{array}$ & $\begin{array}{c}\text { Soll } \\
\text { Moisture } \\
\text { Tenslon } \\
\left(\mathrm{C} \mathrm{m} \mathrm{H}_{2} \mathrm{O}\right) \\
\end{array}$ \\
\hline Date & Time & Days' & $\mathrm{Cl}^{\circ}$ & $B 1^{\circ}$ & $A 1^{a}$ & $\mathrm{C2}^{\mathrm{b}}$ & $\mathrm{B2}^{\mathrm{b}}$ & $A 2^{b}$ & $\mathrm{C3}^{\mathrm{C}}$ & $\mathrm{B3}^{\mathrm{C}}$ & $A 3^{C}$ & $\mathrm{CA}^{\mathrm{d}}$ & $B 4^{d}$ & $\mathrm{A4}^{\mathrm{d}}$ & $C 5^{\circ}$ & $B 6^{\circ}$ & $A 6^{\circ}$ & $\mathrm{Cb}^{\prime}$ & $B 6^{\prime}$ & $A 6^{\prime}$ \\
\hline (1) & $20: 00$ & 29.417 & 192.5 & & 172.6 & & 121.1 & & 185.6 & 180.7 & 181.2 & 164.8 & 144.8 & 147.5 & 168.0 & 151.3 & 150.1 & 189.7 & & \\
\hline 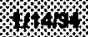 & $22: 00$ & 29.500 & 189.1 & & 170.0 & & 119.4 & & 182.3 & 175.5 & 180.4 & 183.1 & 144.0 & 145.0 & 165.3 & 149.7 & 148.5 & 186.3 & & \\
\hline \% & \begin{tabular}{|l|l}
$0: 00$ \\
\end{tabular} & 29.603 & 184.8 & & 170.1 & & 116.8 & & 175.4 & 171.1 & 174.4 & 157.1 & 140.6 & 145.0 & 161.8 & 148.0 & 146.8 & 183.8 & & \\
\hline 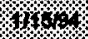 & $2: 00$ & 29.607 & 184.8 & & $\$ 70.1$ & & 116.9 & & 171.9 & 167.6 & 171.8 & 156.2 & 144.0 & 145.9 & 161.0 & 148.0 & 146.8 & 182.0 & & \\
\hline 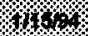 & $4: 00$ & 29.760 & 184.8 & & 170.1 & & 113.4 & & 171.8 & 164.9 & 168.3 & 152.7 & 141.4 & 147.8 & 160.1 & 148.8 & 146.8 & 181.1 & & \\
\hline 1450 & $6: 00$ & 29.833 & 188.6 & & 170.8 & & 114.3 & & 171.8 & 163.1 & 169.2 & 155.3 & 143.2 & 148.5 & 181.8 & 149.7 & 148.5 & 182.8 & & \\
\hline $141 \%$ & $8: 00$ & 29.917 & 184.8 & & 171.0 & & 113.4 & & 171.9 & 161.4 & 168.6 & 152.7 & 142.3 & 145.0 & 161.8 & 148.0 & 146.8 & 181.2 & & \\
\hline 8018 & $10: 00$ & 30.000 & 178.7 & & 171.8 & & 108.2 & & & 148.1 & 154.4 & 143.1 & 140.5 & 145.0 & 156.8 & 144.5 & 144.2 & 173.2 & & \\
\hline 14 1 & 12:00 & 30.083 & 171.7 & & 171.7 & & 98.6 & & & 131.2 & 138.6 & 133.4 & 140.4 & 144.9 & 151.3 & 141.7 & 144.0 & 186.0 & & \\
\hline 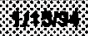 & $14: 00$ & 30.167 & 178.5 & & 178.8 & & 95.1 & & & 134.7 & 142.1 & 135.8 & 146.3 & 148.3 & 153.7 & 150.2 & 149.8 & 168.7 & & \\
\hline $8 / 4 \%$ & \begin{tabular}{|l|}
$16: 00$ \\
\end{tabular} & 30.250 & 189.7 & & 176.8 & & 96.8 & & 171.5 & 156.8 & 158.8 & 149.0 & 152.4 & 151.8 & 181.4 & 158.3 & 153.3 & 178.0 & & \\
\hline 10189 & \begin{tabular}{|l|}
$18: 00$ \\
\end{tabular} & 30.393 & 194.2 & & 178.8 & & 102.0 & & 182.9 & 171.8 & 170.7 & 159.5 & 150.7 & 151.0 & 165.8 & 155.5 & 154.3 & 188.0 & & \\
\hline 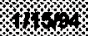 & $20: 00$ & 30.417 & 191.7 & & 174.3 & & 105.5 & & 183.0 & 172.7 & 174.3 & 160.4 & 148.5 & 147.5 & 186.0 & 152.2 & 151.8 & 187.1 & & \\
\hline $4 \%$ \% & $22: 00$ & 30.500 & 190.8 & & 173.5 & & 106.4 & & 180.5 & 171.8 & 174.3 & 160.5 & 148.5 & 148.4 & 166.1 & 152.2 & 151.0 & 187.2 & & \\
\hline 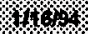 & \begin{tabular}{|l|l|}
$0: 00$ \\
\end{tabular} & 30.583 & 188.3 & & 171.8 & & 107.3 & & 178.8 & 170.2 & 173.5 & 1559.7 & 143.1 & 148.5 & 163.6 & 150.6 & 150.2 & 188.4 & & \\
\hline 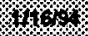 & $2: 00$ & 30.607 & 188.3 & & 173.6 & & 106.5 & & 178.9 & 169.3 & 173.5 & 159.7 & 144.0 & 148.5 & 163.6 & 152.3 & 150.2 & 183.8 & & \\
\hline \% & $4: 00$ & $\mathbf{3 0 . 7 5 0}$ & 186.6 & & 172.7 & & 108.5 & & 175.4 & 165.8 & 170.1 & 158.2 & 144.9 & 145.0 & 161.8 & 148.9 & 148.5 & 182.9 & & \\
\hline (16) & $8: 00$ & 30.833 & 187.5 & & 172.7 & & 106.5 & & 175.4 & 164.9 & 170.9 & 156.2 & 144.1 & 148.5 & 181.8 & 150.6 & 150.3 & 183.8 & & \\
\hline $4 \% 84$ & $8: 00$ & 30.917 & 188.6 & & 171.0 & & 105.7 & & 174.5 & 161.4 & 169.2 & 155.3 & 140.6 & 145.9 & 181.8 & 148.0 & 146.8 & 184.7 & & \\
\hline 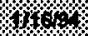 & $10: 00$ & 31.000 & $\begin{array}{l}173.5 \\
\end{array}$ & & 170.8 & & 91.8 & & & 131.3 & 134.4 & 138.7 & 137.9 & 145.0 & 154.0 & 138.3 & 140.6 & 168.8 & & \\
\hline 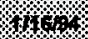 & \begin{tabular}{|l|}
$12: 00$ \\
\end{tabular} & 31.083 & 168.1 & & 177.7 & & 83.9 & & & 117.1 & 125.6 & 127.2 & 141.2 & 148.4 & 147.7 & 145.1 & 147.4 & 160.7 & & \\
\hline 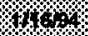 & $14: 00$ & 31.167 & 187.2 & & 176.8 & & 93.4 & & 175.1 & 156.8 & 158.5 & 156.0 & 150.7 & 148.3 & 102.4 & 159.0 & 156.8 & 181.6 & & \\
\hline 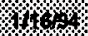 & $16: 00$ & 31.250 & 195.0 & & 180.2 & & 100.2 & & 181.9 & 168.2 & 169.8 & 162.8 & 154.1 & 156.2 & 188.3 & 163.2 & 158.5 & 180.4 & & \\
\hline אut & $18: 00$ & 31.333 & 200.3 & & 177.7 & & 108.1 & & 190.7 & 181.5 & 183.8 & 188.2 & 152.5 & 154.5 & 172.8 & 162.5 & 159.4 & 194.8 & & \\
\hline 10064 & $20: 00$ & 31.417 & 196.0 & & 174.4 & & 115.1 & & 189.2 & 183.4 & 187.3 & 168.3 & 147.4 & 151.1 & 173.0 & 155.7 & 154.5 & 191.5 & & \\
\hline 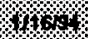 & $22: 00$ & 31.500 & 193.5 & & 173.5 & & 123.8 & & 185.8 & 183.4 & 187.4 & 166.7 & 148.4 & 149.4 & 171.4 & 156.7 & 153.7 & 193.4 & & \\
\hline 174 & $0: 00$ & 31.503 & 192.7 & & 173.8 & & 125.5 & & 185.8 & 182.6 & 187.4 & 166.7 & 145.8 & 148.5 & 169.7 & 154.1 & 152.8 & 194.4 & & \\
\hline \% & $2: 00$ & 31.667 & 181.0 & & 172.8 & & 127.3 & & 181.6 & 179.1 & 184.9 & 165.9 & 145.0 & 148.6 & 188.1 & 153.3 & 152.1 & 180.1 & & \\
\hline $4 \% \%$ & $4: 00$ & 31.760 & 189.3 & & 171.8 & & 123.9 & & 179.0 & 175.6 & 182.3 & 163.3 & 145.1 & 149.5 & 165.5 & 150.8 & 151.3 & 187.5 & & \\
\hline$\%$, & $6: 00$ & 31.833 & 188.4 & & 172.8 & & 123.0 & & 178.1 & 175.6 & 180.6 & 103.3 & 145.8 & 148.6 & 168.4 & 152.5 & 151.3 & 187.5 & & \\
\hline$\%$ & $8: 00$ & 31.817 & 185.8 & & 171.4 & & 120.4 & & 177.3 & 165.9 & 178.2 & 180.7 & 141.6 & 147.8 & 165.5 & 148.2 & 147.8 & 186.7 & & \\
\hline$\% 17 \%$ & $10: 00$ & 32,000 & 175.3 & & 171.8 & & 110.0 & & 162.4 & 940.2 & 144.8 & 145.7 & 140.6 & 147.7 & 157.6 & 141.0 & 141.7 & 173.3 & & \\
\hline 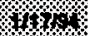 & $12: 00$ & 32.083 & 168.2 & & 177.8 & & 101.2 & & & 123.3 & 128.3 & 129.0 & 141.3 & 150.2 & 149.6 & 145.2 & 148.3 & 102.8 & & \\
\hline 1 $1 \%$ & $14: 00$ & 32.107 & 178.7 & & 180.3 & & 100.2 & & & 132.9 & 142.1 & 138.5 & 154.1 & 751.8 & 154.5 & 157.2 & 155.8 & 188.4 & & \\
\hline 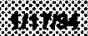 & $16: 00$ & 32.260 & 195.6 & & 181.8 & & 102.7 & & 173.9 & 156.6 & 158.4 & 150.6 & 158.1 & 159.5 & 185.4 & 165.5 & 180.7 & 181.2 & & \\
\hline 1240 & $\begin{array}{l}18: 00 \\
\end{array}$ & 32.333 & 208.1 & & 181.0 & & 113.0 & & 193.8 & 182.2 & 183.6 & 168.9 & 160.8 & 157.8 & 175.0 & 168.2 & 164.3 & 189.1 & & \\
\hline 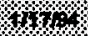 & $20: 00$ & 32.417 & 189.4 & & 177.7 & & 117.6 & & 191.6 & 183.3 & 188.1 & 169.1 & 151.6 & 154.5 & 172.8 & 150.0 & 157.7 & 195.7 & & \\
\hline 40 & $22: 00$ & 32.500 & 196.0 & & 176.1 & & 121.1 & & 189.1 & 182.5 & 187.3 & 168.3 & 150.0 & 151.8 & 172.1 & 155.7 & 158.1 & 183.2 & & \\
\hline
\end{tabular}


Table 5. Tensiometer Data

\begin{tabular}{|c|c|c|c|c|c|c|c|c|c|c|c|c|c|c|c|c|c|c|c|c|}
\hline & & & & & & & & & Ten & slometer & $\overline{\text { Data }}$ & & & & & & & & & \\
\hline & & Test & $\begin{array}{c}\text { Soll } \\
\text { Molsture } \\
\text { Tension } \\
\left(\mathrm{Cm} \mathrm{H}_{2} \mathrm{O}\right)\end{array}$ & $\begin{array}{c}\text { Soll } \\
\text { Molsture } \\
\text { Tension } \\
\left(\mathrm{Cm} \mathrm{H}_{2} \mathrm{O}\right)\end{array}$ & $\begin{array}{l}\text { Soll } \\
\text { Moisture } \\
\text { Tension } \\
\left(\mathrm{Cm} \mathrm{H}_{2} \mathrm{O}\right)\end{array}$ & $\begin{array}{c}\text { Soll } \\
\text { Moisture } \\
\text { Tension } \\
\left(\mathrm{Cm} \mathrm{H}_{2} \mathrm{O}\right)\end{array}$ & $\begin{array}{c}\text { Soll } \\
\text { Molsture } \\
\text { Tension } \\
\text { (Cm } \mathrm{H}_{2} \mathrm{O} \text { ) }\end{array}$ & $\begin{array}{c}\text { Soll } \\
\text { Molsture } \\
\text { Tension } \\
\left(\mathrm{cm} \mathrm{H}_{2} \mathrm{O}\right)\end{array}$ & $\begin{array}{l}\text { Soll } \\
\text { Moisture } \\
\text { Tension } \\
\left(\mathrm{Cm} \mathrm{H}_{2} \mathrm{O} \text { ) }\right.\end{array}$ & $\begin{array}{c}\text { Soll } \\
\text { Molsture } \\
\text { Tension } \\
\left(\mathrm{cm} \mathrm{H}_{2} \mathrm{O}\right)\end{array}$ & $\begin{array}{c}\text { Soll } \\
\text { Molsture } \\
\text { Tension } \\
\left(\mathrm{cm} \mathrm{H}_{2} \mathrm{O}\right)\end{array}$ & $\begin{array}{c}\text { Soll } \\
\text { Molsture } \\
\text { Tension } \\
\left(\mathrm{Cm} \mathrm{H}_{2} \mathrm{O}\right)\end{array}$ & $\begin{array}{c}\text { Soll } \\
\text { Moisture } \\
\text { Tension } \\
\left(\mathrm{Cm} \mathrm{H}_{2} \mathrm{O}\right)\end{array}$ & $\begin{array}{c}\text { Soll } \\
\text { Molsture } \\
\text { Tenslon } \\
\left(\mathrm{cm} \mathrm{H}_{2} \mathrm{O}\right)\end{array}$ & $\begin{array}{c}\text { Soll } \\
\text { Molsture } \\
\text { Tension } \\
\left(\mathrm{cm} \mathrm{H}_{2} \mathrm{O}\right)\end{array}$ & $\begin{array}{c}\text { Soil } \\
\text { Moisture } \\
\text { Tansion } \\
\left(\mathrm{Cm} \mathrm{H}_{2} \mathrm{O}\right)\end{array}$ & $\begin{array}{c}\text { Soll } \\
\text { Moisture } \\
\text { Tension } \\
\text { (em Hz) }\end{array}$ & $\begin{array}{c}\text { Soll } \\
\text { Molsture } \\
\text { Tension } \\
\left(\mathrm{cm} \mathrm{H}_{2} \mathrm{O}\right)\end{array}$ & $\begin{array}{c}\text { Soll } \\
\text { Molstur } \\
\text { Tension } \\
\left(\mathrm{cm} \mathrm{H}_{2} \mathrm{O}\right)\end{array}$ & 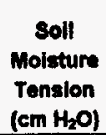 \\
\hline Date & Time & Days ${ }^{1}$ & $C 1^{*}$ & $\mathrm{B1}^{\circ}$ & $A 1^{a}$ & $C 2^{b}$ & $B 2^{6}$ & $A 2^{b}$ & $\mathrm{C} 3^{\mathrm{c}}$ & $83^{6}$ & $A 3^{2}$ & $\mathrm{C4}^{\mathrm{d}}$ & $B 4^{d}$ & $A 4^{d}$ & \begin{tabular}{|c|}
$\mathrm{Cs}^{\circ}$ \\
\end{tabular} & $85^{\circ}$ & $A 5^{\circ}$ & $\mathrm{C6}^{\prime}$ & $86^{\prime}$ & $A G^{\prime}$ \\
\hline W1 1 . & $0: 00$ & 32.683 & 192.6 & & 175.2 & & 122.0 & & 185.7 & 180.7 & 186.5 & 167.5 & 149.2 & 152.0 & 168.7 & 156.6 & 153.6 & 182.4 & & \\
\hline$\%$ & $2: 00$ & 32.667 & 192.7 & & 174.4 & & 123.8 & & 183.2 & 178.0 & 183.9 & 186.7 & 147.5 & 152.0 & 169.6 & 155.0 & 153.7 & 189.8 & & \\
\hline itis & $4: 00$ & 32.750 & 190.9 & & 173.6 & & 122.1 & & 182.4 & 175.5 & 183.1 & 163.2 & 147.6 & 151.2 & 166.2 & 154.1 & 152.9 & 189.1 & & \\
\hline mo & $6: 00$ & 32.033 & 191.0 & & 173.6 & & 120.4 & & 181.5 & 175.5 & 180.5 & 163.2 & 147.8 & 151.2 & 168.0 & 153.3 & 152.9 & 100.0 & & \\
\hline in & $8: 00$ & 32.917 & 187.5 & & 172.8 & & 116.9 & & 178.1 & 168.5 & 174.4 & 159.7 & 144.1 & 148.6 & 168.3 & 149.0 & 151.2 & 185.7 & & \\
\hline $116 \%$ & $10: 00$ & 33.000 & 175.2 & & 173.5 & & 105.8 & & 163.2 & 137.5 & 144.0 & 145.7 & 141.4 & 448.5 & 155.7 & 140.0 & 142.4 & 172.3 & & \\
\hline 1118. & $12: 00$ & 33.083 & 180.2 & & 180.3 & & 103.7 & & 175.1 & 140.0 & 129.9 & 154.3 & 153.3 & 151.8 & 152.0 & 153.7 & 149.9 & 185.1 & & \\
\hline ik & $14: 00$ & 33.167 & 172.2 & & 181.9 & & 94.9 & & & 125.7 & 137.6 & 127.9 & 151.3 & 150.8 & 153.4 & 155.2 & 158.5 & 160.3 & & \\
\hline KI:16\% & $16: 00$ & 33.250 & 197.2 & & 186.0 & & 89.1 & & 172.8 & 155.6 & 159.2 & 148.7 & 161.4 & 161.2 & 167.8 & 168.8 & 163.1 & 479.2 & & \\
\hline 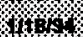 & $18: 00$ & 33.333 & 211.2 & & 181.8 & & 109.8 & & 198.4 & 186.8 & 186.1 & 169.7 & 165.1 & 160.4 & 179.2 & 170.8 & 168.8 & 197.8 & & \\
\hline 8. & $20: 00$ & 33.417 & 205.5 & & 177.7 & & 118.4 & & 197.6 & 189.4 & 192.4 & 173.5 & 155.1 & 154.5 & 178.2 & 182.5 & 161.2 & 189.2 & & \\
\hline \% & $22: 00$ & 33.500 & 199.5 & & 177.8 & & 118.5 & & 192.6 & 186.0 & 189.9 & 170.9 & 150.9 & 152.8 & 173.0 & 157.4 & 157.0 & 185.8 & & \\
\hline (x) & $0: 00$ & 33.683 & 194.4 & & 178.1 & & 120.3 & & 189.2 & 183.4 & 187.4 & 169.2 & 150.8 & 152.0 . & 172.2 & 155.8 & 155.4 & 193.4 & & \\
\hline His & $2: 00$ & 33.667 & 193.5 & & 177.1 & & 119.5 & & 186.7 & 181.7 & 187.4 & 168.7 & 149.3 & 152.0 & 169.7 & 156.7 & 155.5 & 192.6 & & \\
\hline 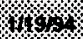 & $4: 00$ & 33.750 & 192.7 & & 177.1 & & 118.5 & & 184.1 & 179.1 & 186.6 & 186.7 & 148.5 & 152.8 & 169.7 & 155.8 & 154.8 & 180.0 & & \\
\hline $119 \%$ & $6: 00$ & $\mathbf{3 3 . 8 3 3}$ & 192.8 & & 175.4 & & 116.1 & & 183.3 & 177.3 & 184.0 & 165.0 & 148.5 & 152.1 & 169.8 & 156.8 & 154.7 & 189.2 & & \\
\hline 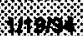 & $8: 00$ & 33.917 & 190.1 & & 175.4 & & 115.2 & & 180.7 & 172.0 & 178.8 & 163.3 & 145.9 & 151.2 & 168.8 & 151.6 & 152.1 & 188.3 & & \\
\hline 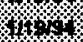 & $10: 00$ & 34.000 & 175.2 & & 174.4 & & 102.2 & & & 137.5 & 143.1 & 143.1 & 139.7 & 148.5 & 158.3 & 138.3 & 140.7 & 172.3 & & \\
\hline 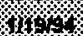 & $12: 00$ & 34.083 & 167.2 & & 183.8 & & 90.8 & & & 115.3 & 128.2 & 128.1 & 140.3 & 152.7 & 149.4 & 141.8 & 149.9 & 159.8 & & \\
\hline (2) & 14:00 & 34.167 & 174.8 & & 184.4 & & 24.9 & 88.2 & & 151.3 & 142.8 & 141.0 & 165.1 & 154.3 & 153.4 & 158.6 & 158.2 & 172.4 & & \\
\hline \%ing & $16: 00$ & 34.250 & 193.7 & & 186.8 & 180.7 & 103.4 & 88.1 & 172.8 & 172.3 & 155.6 & 145.2 & 160.5 & 162.0 & 167.7 & 164.4 & 163.0 & 179.1 & & \\
\hline 1.op & $18: 00$ & 34.333 & 209.5 & & 184.4 & 189.8 & 114.7 & & 199.0 & 192.7 & 184.4 & 169.7 & 164.2 & 162.1 & 181.8 & 165.5 & 168.5 & 198.6 & & \\
\hline \% & $20: 00$ & 34.417 & 206.3 & & 180.3 & 186.4 & 121.0 & & 200.2 & 183.2 & 189.8 & 174.3 & 155.0 & 158.0 & 178.8 & 159.8 & 162.8 & 199.2 & & \\
\hline \% & $22: 00$ & 34.600 & 202.1 & & 179.6 & 172.7 & 126.3 & & 196.8 & 179.8 & 190,8 & 174.4 & 151.7 & 156.3 & 175.6 & 158.3 & 160.5 & 198.5 & & \\
\hline $1000 \%$ & $0: 00$ & 34.683 & 199.6 & & 177.9 & & 130.7 & & 193.6 & 175.5 & 180.8 & 174.5 & 150.1 & 155.5 & 174.8 & 957.8 & 158.0 & 196.8 & & \\
\hline 120,4 & $2: 00$ & 34.667 & 196.2 & 144.3 & 177.1 & & 134.2 & & 190.2 & 172.0 & 180.9 & 171.1 & 148.4 & 155.6 & 174.1 & 156.8 & 157.2 & 194.4 & & \\
\hline $1,20.4$ & $4: 00$ & 34.750 & 194.5 & 136.5 & 176.2 & & 135.8 & & 186.7 & 172.0 & 190.1 & 170.2 & 148.5 & 155.6 & 172.3 & 158.5 & 158.1 & 193.5 & & \\
\hline , & $6: 00$ & 34.833 & 196.2 & 141.7 & 178.0 & & 138.5 & & 185.8 & 172.0 & 189.2 & 170.2 & 150.2 & 154.7 & 172.4 & 157.7 & 159.0 & 193.6 & & \\
\hline 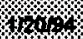 & $8: 00$ & 34.017 & 191.8 & 152.2 & 177.2 & & 140.3 & & 184.2 & 167.6 & 184.9 & 167.6 & 145.0 & 153.9 & 970.7 & 154.2 & 154.7 & 181.0 & & \\
\hline$\sqrt{301} \%$ & $10: 00$ & 36.000 & 177.0 & & 177.1 & & 127.2 & & 161.5 & 141.0 & 149.2 & 145.7 & 141.4 & 152.0 & 160.1 & 142.7 & 142.5 & 176.8 & & \\
\hline (123) & $12: 00$ & 36.083 & 168.4 & & 182.1 & & 118.3 & & & 140.0 & 130.0 & 132.5 & 143.8 & 154.5 & 150.4 & 148.6 & 151.7 & 163.3 & & \\
\hline$\%$ & $14: 00$ & 36.167 & 172.2 & 136.1 & 187.1 & & 115.6 & & & 151.3 & 135.0 & 130.5 & 157.4 & 155.2 & 155.1 & 164.8 & 159.1 & 164.6 & & \\
\hline nong & $18: 00$ & 36.250 & 203.2 & & 189.4 & & 120.6 & & & 185.5 & 162.6 & 154.7 & 168.5 & 168.1 & 172.0 & 173.0 & 168.8 & 186.0 & & \\
\hline $180 \%$ & $18: 00$ & 96.333 & 216.3 & 131.6 & 186.1 & & 131.0 & & 186.7 & 204.1 & 191.2 & 177.5 & 167.6 & 165.5 & 186.0 & 172.4 & 172.7 & 205.5 & & \\
\hline 8 & $20: 00$ & 36.417 & 209.7 & & 181.1 & & 133.8 & & 193.2 & 196.4 & 182.3 & 183.0 & 157.5 & 158.6 & 178.5 & 162.3 & 163.6 & 208.8 & & \\
\hline \% & $22: 00$ & 36.500 & 202.0 & 131.8 & $184: 2$ & & 136.5 & & 191.6 & 186.8 & 182.4 & 177.8 & 153.3 & 158.0 & 178.0 & 159.9 & 101.2 & 198.3 & & \\
\hline 28 & $0: 00$ & 36.583 & 202.1 & 129.4 & 180.4 & & 140.9 & & 198.0 & 185.1 & 198.8 & 177.0 & 154.3 & 158.9 & 178.1 & 161.8 & 101.3 & 199.3 & & \\
\hline 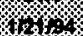 & $2: 00$ & 35.667 & 199.6 & 134.7 & 180.5 & & 144.5 & & 198.2 & 182.5 & 197.8 & 177.1 & 154.4 & 159.0 & 178.5 & 161.9 & 161.5 & 186.8 & & \\
\hline
\end{tabular}


Table 5. Tensiometer Data

\begin{tabular}{|c|c|c|c|c|c|c|c|c|c|c|c|c|c|c|c|c|c|c|c|c|}
\hline & & & & & & & & & Ten: & iometer & Data & & & & & & & & & \\
\hline & & Test & $\begin{array}{c}\text { Soll } \\
\text { Molsture } \\
\text { Tension } \\
\left(\mathrm{Cm} \mathrm{H}_{2} \mathrm{O}\right)\end{array}$ & $\begin{array}{c}\text { Soll } \\
\text { Moisture } \\
\text { Tension } \\
\left(\mathrm{Cm}^{\left.\mathrm{H}_{2} \mathrm{O}\right)}\right. \\
\end{array}$ & $\begin{array}{c}\text { Soll } \\
\text { Molsture } \\
\text { Tension } \\
\left(\mathrm{c} \mathrm{H}_{2} \mathrm{O}\right)\end{array}$ & $\begin{array}{c}\text { Soll } \\
\text { Moisture } \\
\text { Tension } \\
\left(\mathrm{Cm} \mathrm{H}_{2} \mathrm{O}\right) \\
\end{array}$ & $\begin{array}{c}\text { Soll } \\
\text { Molsture } \\
\text { Tension } \\
\left(\mathrm{Cm} \mathrm{H}_{2} \mathrm{O}\right) \\
\end{array}$ & $\begin{array}{c}\text { Soll } \\
\text { Molsture } \\
\text { Tension } \\
\left(\mathrm{Cm} \mathrm{H}_{2} \mathrm{O} \text { ) }\right.\end{array}$ & $\begin{array}{c}\text { Soll } \\
\text { Molsture } \\
\text { Tension } \\
\left(\mathrm{Cm} \mathrm{H}_{2} \mathrm{O}\right) \\
\end{array}$ & $\begin{array}{c}\text { Soll } \\
\text { Moisture } \\
\text { Tension } \\
\left(\mathrm{Cm} \mathrm{H}_{2} \mathrm{O}\right) \\
\end{array}$ & $\begin{array}{c}\text { Soll } \\
\text { Moisture } \\
\text { Tension } \\
\left(\mathrm{cm} \mathrm{H} \mathrm{H}_{2} \mathrm{O}\right)\end{array}$ & $\begin{array}{c}\text { soll } \\
\text { Moisture } \\
\text { Tension } \\
\left(\mathrm{Cm} \mathrm{H}_{2} \mathrm{O}\right)\end{array}$ & $\begin{array}{c}\text { Soll } \\
\text { Molisture } \\
\text { Tonsion } \\
\left(\mathrm{Cm} \mathrm{H}_{2} \mathrm{O}\right)\end{array}$ & $\begin{array}{c}\text { Soll } \\
\text { Molsture } \\
\text { Tension } \\
\left(\mathrm{Cm} \mathrm{H}_{2} \mathrm{O}\right)\end{array}$ & $\begin{array}{c}\text { soll } \\
\text { Molisture } \\
\text { Tension } \\
\left(\mathrm{cm} \mathrm{H}_{2} \mathrm{O}\right) \\
\end{array}$ & $\begin{array}{c}\text { Soll } \\
\text { Molsture } \\
\text { Tension } \\
\left(\mathrm{Cm} \mathrm{H}_{2} \mathrm{O}\right)\end{array}$ & $\begin{array}{c}\text { Soll } \\
\text { Molature } \\
\text { Tension } \\
\text { (em HzO) }\end{array}$ & $\begin{array}{c}\text { Soll } \\
\text { Molsture } \\
\text { Tension } \\
\left(\mathrm{Cm} \mathrm{H}_{2} \mathrm{O}\right) \\
\end{array}$ & $\begin{array}{c}\text { Soll } \\
\text { Molature } \\
\text { Tension } \\
\left(\mathrm{Cm} \mathrm{H}_{2} \mathrm{O}\right)\end{array}$ & $\begin{array}{c}\text { Soll } \\
\text { Moliture } \\
\text { Tension } \\
(\mathrm{cm} \mathrm{H} \text {,O) }\end{array}$ \\
\hline Date & Time & Days' & $\mathrm{C1}^{2}$ & $B 1^{2}$ & $A 1^{*}$ & $\mathrm{C2}^{6}$ & $B 2^{b}$ & $A 2^{b}$ & $\mathrm{C3}^{\mathrm{C}}$ & $\mathrm{B3}^{\mathrm{C}}$ & $A 3^{c}$ & $\mathrm{CA}^{\mathrm{d}}$ & $B 4^{d}$ & $A 4^{d}$ & $C 5^{\circ}$ & $\mathrm{BS}^{\circ}$ & $A 5^{\circ}$ & $\mathrm{Cb}^{\prime}$ & $\mathrm{BG}^{\prime}$ & $A 6^{f}$ \\
\hline (1) & A:00 & 36.750 & 197.9 & 136.5 & 178.8 & & 148.0 & & 194.5 & 178.2 & 194.4 & 173.7 & 150.2 & 155.5 & 174.0 & 159.4 & 158.9 & 197.0 & & \\
\hline $121 \%$ & $6: 00$ & 36.833 & 196.2 & 140.0 & 178.0 & & 148.9 & & 193.7 & 178.4 & 195.3 & 173.7 & 952.0 & 157.3 & 173.2 & 159.5 & 159.0 & 196,2 & & \\
\hline $12 \%$ & $8: 00$ & 35.917 & 191.8 & 136.5 & 177.2 & & 168.0 & & 189.4 & 170.3 & 180.1 & 170.3 & 147.7 & 154.7 & 171.6 & 158.0 & 155.8 & 193.6 & & \\
\hline rishor & $10: 00$ & 36.000 & 175.3 & 133.0 & 177.1 & & 137.6 & & & 138.4 & 152.7 & 149.2 & 140.6 & 152.1 & 161.8 & 142.8 & 143.4 & 178.6 & & \\
\hline $4 \%$ & $12: 00$ & 36.083 & 163.0 & & 183.0 & & 128.0 & & & 130.4 & 130.0 & 127.3 & 144.7 & 154.6 & 149.6 & 148.7 & 150.8 & 160.8 & & \\
\hline 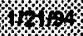 & $14: 00$ & 36.167 & 178.7 & & 187.2 & & 126.9 & & & 156.7 & 145.5 & 138.5 & 161.0 & 157.0 & 158.8 & 171.0 & 163.8 & 170.1 & & \\
\hline 140 & $16: 00$ & 30.250 & 200.6 & & 189.4 & & 130.1 & & 171.8 & 178.5 & 160.0 & 155.6 & 164.8 & 168.1 & 172.0 & 172.2 & 167,4 & 185.2 & & \\
\hline 1114 & $\begin{array}{l}18: 00 \\
\end{array}$ & 36.333 & 218.5 & & 187.1 & & 139.8 & & 202.6 & 202.5 & 190.5 & 179.4 & 170.4 & 168.6 & 185.4 & 172.7 & 173.0 & 205.8 & & \\
\hline $114 \%$ & $20: 00$ & 36.417 & 209.9 & 132.8 & 183.0 & & 144.4 & & 208.1 & 197.4 & 194.2 & 181.4 & 155.2 & 159.8 & 182.4 & 164.4 & 184.7 & 203.7 & & \\
\hline 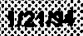 & $22: 00$ & 36.500 & 203.9 & 142.5 & 181.4 & & 148.8 & & 208.8 & 191.4 & 194.3 & 180.6 & 154.4 & 159.0 & 179.1 & 161.9 & 162.3 & 203.0 & & \\
\hline $10 \% 2 \%$ & $0: 00$ & 36.583 & 198.8 & 144,3 & 180.8 & & 148.8 & & 203.2 & 188.1 & 194.4 & 177.2 & 151.0 & 158.2 & 177.5 & 161.1 & 161.5 & 198.6 & & \\
\hline 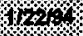 & $2: 00$ & 36.667 & 196.2 & 142.6 & 179.7 & & 150.8 & & 199.7 & 181.7 & 194.4 & 175.5 & 151.9 & 159.1 & 174.9 & 159.4 & 159.8 & 197.0 & & \\
\hline 4514 & $4: 00$ & 36.750 & 199.2 & 146.1 & 179.8 & & 1524 & & 198.1 & 178.1 & 191.8 & 173.7 & 151.1 & 158.2 & 173.2 & 159.4 & 158.1 & 195.3 & & \\
\hline$\% 1 \%$ & $6: 00$ & 36.833 & 195.4 & 149.5 & 178.9 & & 184.4 & & 196.3 & 177.3 & 191.0 & 173.7 & 152.0 & 158.2 & 173.3 & 159.5 & 161.6 & 194.5 & & \\
\hline 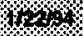 & $8: 00$ & 36.917 & 192.8 & 148.1 & 178.0 & & 147.2 & & 192.0 & 172.0 & 188.4 & 170.3 & 148.5 & 158.2 & 173.3 & 156.0 & 157.3 & 192.8 & & \\
\hline $12 \%$ \% & $10: 00$ & 37.000 & 174.4 & 139.0 & 177.9 & & 140.2 & & 164.9 & 141.0 & 152.7 & 148.3 & 141.4 & 152.0 & 160.8 & 144.4 & 146.7 & 177.6 & & \\
\hline 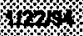 & $12: 00$ & 37.083 & 162.8 & 132.8 & 983.8 & & 130.5 & & 140.4 & 129.4 & 130.0 & 128.8 & 143.8 & 155.4 & 150.3 & 147.7 & 150.8 & 164.2 & & \\
\hline $10 \% 1 \%$ & $14: 00$ & 37.167 & 178.1 & 134.2 & 190.3 & & 126.7 & & 143.4 & 147.7 & 139.2 & 133.8 & 184.0 & 162.0 & 158.3 & 169.7 & 102.2 & 166.8 & & \\
\hline 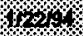 & 18:00 & 37.260 & 201.3 & 132.3 & 192.8 & & 129.9 & & 171.7 & 180.1 & 159.9 & 155.5 & 168.1 & 169.7 & 173.5 & 178.1 & 169.7 & 186.7 & & \\
\hline 1) & \begin{tabular}{|l|}
$18: 00$ \\
\end{tabular} & 37.333 & 217.4 & 139.8 & 188.0 & & 140.7 & & 202.6 & 203.4 & 187.8 & 178.5 & 171.3 & 168.3 & 188.3 & 178.1 & 173.8 & 204.8 & & \\
\hline 1721 & $20: 00$ & 37.417 & 210.7 & 139.8 & 183.8 & & 144.3 & & 208.1 & 201.8 & 194.2 & 181.3 & 158.8 & 162.4 & 183.2 & 168.0 & 167.3 & 200.2 & & \\
\hline 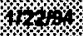 & $22: 00$ & 37.600 & 203.8 & & 183.8 & & 147.8 & & 207.3 & 194.8 & 194.2 & 179.7 & 156.1 & 161.5 & 179.8 & 163.5 & 163.8 & 202.8 & & \\
\hline 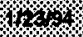 & $0: 00$ & 37.683 & 199.5 & 134.6 & 181.3 & & 147.9 & & 203.0 & 189.6 & 194.3 & 177.1 & 154.4 & 159.0 & 177.3 & 163.6 & 102.2 & 200.3 & & \\
\hline $1 \%$ & $2: 00$ & 37.667 & 195.2 & & 181.4 & & 147.8 & & 196.1 & 181.6 & 189.9 & 173.6 & 150.9 & 159.0 & 173.8 & 180.1 & 160.5 & 193.3 & & \\
\hline $1272 \%$ & $4: 00$ & 37.760 & 197.0 & & 181.4 & & 148.8 & & 197.9 & 181.6 & 190.8 & 173.6 & 155.3 & 161.6 & 175.7 & 962.8 & 102.3 & 196.8 & & \\
\hline $5120 \%$ & $8: 00$ & 37.233 & 197.0 & & 181.4 & & 151.4 & & 197.1 & 179.8 & 190.9 & 173.7 & 154.5 & 159.9 & 178.6 & 162.0 & 181.5 & 197.0 & & \\
\hline $1723 \%$ & $8: 00$ & 37.917 & 192.7 & & 180.6 & & 175.7 & & 193.7 & 176.4 & 188.3 & 171.1 & 151.1 & 158.2 & 174.1 & 159.4 & 159.8 & 195.3 & & \\
\hline 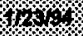 & $10: 00$ & 38.000 & 175.2 & & 180.4 & & 137.5 & & & 143.6 & 152.6 & 147.4 & 140.4 & 154.6 & 161.7 & 143.5 & 146.6 & 177.5 & & \\
\hline 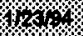 & $12: 00$ & 38.083 & 157.6 & & 184.6 & & 127.8 & & & 125.8 & 125.6 & 124.5 & 147.2 & 158.0 & 148.5 & 148.5 & 151.6 & 159.7 & & \\
\hline 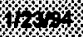 & $14: 00$ & 38.167 & 178.2 & & 191.3 & & 126.7 & & & 147.7 & 141.0 & 134.8 & 163.3 & 163.0 & 161.0 & 171.6 & 164.1 & 168.8 & & \\
\hline 1234 & $16: 00$ & 38.250 & 208.5 & & 194.7 & & 131.0 & & 177.2 & 182.8 & 165.2 & 159.2 & 172.7 & 175.2 & 177.3 & 182.7 & 174.3 & 190.5 & & \\
\hline 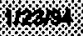 & $18: 00$ & 38.333 & 216.6 & & 188.9 & & 138.1 & & 202.7 & 203.4 & 188.0 & 179.5 & 169.8 & 168.4 & 186.4 & 178.2 & 173.9 & 206.8 & & \\
\hline 12114 & $20: 00$ & 38.417 & 206.3 & & 184.7 & & 140.0 & & 201.1 & 196.5 & 188.1 & 178.9 & 160.2 & 164.1 & 180.5 & 168.0 & 167.2 & 202.6 & & \\
\hline 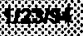 & $22: 00$ & 38.600 & 200.3 & & 184.7 & & 140.0 & & 197.7 & 190.3 & 187.2 & 172.6 & 150.8 & 162.4 & 178.0 & 103.4 & 163.8 & 197.4 & & \\
\hline $14 \%$ & $0: 00$ & 38.503 & 202.8 & & 186.5 & & 141.7 & & 199.4 & 188.6 & 187.2 & 174.3 & 159.4 & 164.1 & 178.9 & 167.8 & 165.5 & 200.1 & & \\
\hline 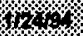 & $2: 00$ & 30.687 & 201.2 & & 183.9 & & 144.4 & & 202.1 & 187.7 & 190.8 & 177.0 & 158.7 & 162.4 & 179.0 & 165.2 & 164.8 & 200.2 & & \\
\hline $171 \%$ & $4: 00$ & 38.750 & 199.5 & & 183.9 & & 144.4 & & 198.5 & 186.0 & 188.4 & 172.7 & 158.8 & 162.4 & 174.7 & 184.4 & 163.9 & 197.6 & & \\
\hline 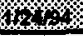 & $6: 00$ & 38.833 & 193.4 & & 183.9 & & 144.4 & & 192.6 & 178.9 & 183.8 & 170.1 & 154.3 & 161.6 & 172.8 & 161.8 & 162.2 & 193.2 & & \\
\hline
\end{tabular}


Table 5. Tensiometer Data

\begin{tabular}{|c|c|c|c|c|c|c|c|c|c|c|c|c|c|c|c|c|c|c|c|c|}
\hline & & & & & & & & & Ten & siometer & Data & & & & & & & & & \\
\hline & & Test & $\begin{array}{c}\text { Soll } \\
\text { Moisture } \\
\text { Tenslon } \\
\text { (cm } \mathrm{H}_{2} \mathrm{O} \text { ) } \\
\end{array}$ & 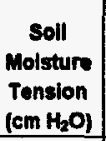 & $\begin{array}{c}\text { Soll } \\
\text { Molsture } \\
\text { Tension } \\
\left(\mathrm{C} \mathrm{H}_{2} \mathrm{O}\right) \\
\end{array}$ & $\begin{array}{c}\text { Soil } \\
\text { Moisture } \\
\text { Tension } \\
\left(\mathrm{Cm} \mathrm{H}_{2} \mathrm{O}\right) \\
\end{array}$ & 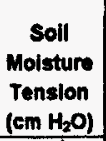 & $\begin{array}{c}\text { Soll } \\
\text { Moisture } \\
\text { Tenslon } \\
\left(\mathrm{Cm} \mathrm{H}_{2} \mathrm{O}\right) \\
\end{array}$ & $\begin{array}{c}\text { Soll } \\
\text { Moisture } \\
\text { Tenslon } \\
\left(\mathrm{Cm} \mathrm{H}_{2} \mathrm{O}\right) \\
\end{array}$ & $\begin{array}{c}\text { Soll } \\
\text { Molsture } \\
\text { Tension } \\
\left(\mathrm{Cm} \mathrm{H}_{2} \mathrm{O}\right) \\
\end{array}$ & $\begin{array}{c}\text { Soll } \\
\text { Molsture } \\
\text { Tension } \\
\text { (cm } \mathrm{H}_{2} \mathrm{O} \text { ) }\end{array}$ & $\begin{array}{c}\text { Soll } \\
\text { Moisture } \\
\text { Tension } \\
\left(\mathrm{Cm} \mathrm{H}_{2} \mathrm{O}\right) \\
\end{array}$ & $\begin{array}{c}\text { Soll } \\
\text { Moisture } \\
\text { Tension } \\
\left(\mathrm{Cm} \mathrm{H}_{2} \mathrm{O}\right) \\
\end{array}$ & $\begin{array}{c}\text { Soll } \\
\text { Moisture } \\
\text { Tension } \\
\left(\mathrm{em} \mathrm{H}_{2} \mathrm{O}\right) \\
\end{array}$ & $\begin{array}{c}\text { Soll } \\
\text { Molsture } \\
\text { Tension } \\
\left(\mathrm{Cm} \mathrm{H}_{2} \mathrm{O}\right) \\
\end{array}$ & $\begin{array}{c}\text { Soll } \\
\text { Molsture } \\
\text { Tenalon } \\
\left(\mathrm{cm} \mathrm{H}_{2} \mathrm{O}\right)\end{array}$ & $\begin{array}{c}\text { Soll } \\
\text { Molsture } \\
\text { Tenslon } \\
\left(\mathrm{cm} \mathrm{H}_{2} \mathrm{O}\right) \\
\end{array}$ & $\begin{array}{c}\text { Soll } \\
\text { molstur } \\
\text { Tension } \\
\left(\mathrm{cm} \mathrm{H}_{2} \mathrm{O}\right)\end{array}$ & $\begin{array}{c}\text { Soll } \\
\text { Molsture } \\
\text { Tonsion } \\
\left(\mathrm{em} \mathrm{H}_{2} \mathrm{O}\right)\end{array}$ & $\begin{array}{c}\text { Soll } \\
\text { Molsture } \\
\text { Tension } \\
\left(\mathrm{Cm} \mathrm{H}_{2} \mathrm{O}\right) \\
\end{array}$ \\
\hline Date & Time & Days' & $C 1^{2}$ & $B 1^{n}$ & $A 1^{2}$ & $\mathrm{C2}^{6}$ & $82^{b}$ & $A 2^{b}$ & $\mathrm{C3}^{\mathrm{e}}$ & $\mathrm{B3}^{\mathrm{C}}$ & $\mathrm{AB}^{\mathrm{C}}$ & $\mathrm{CA}^{\mathrm{d}}$ & $B 4^{d}$ & $\mathbf{A 4}^{\mathrm{d}}$ & $65^{\circ}$ & $85^{\circ}$ & $A 5^{\circ}$ & $\mathrm{Co}^{\prime}$ & $B 6^{f}$ & $A 6^{\prime}$ \\
\hline 14 & $8: 00$ & 38.917 & 193.4 & & 183.9 & & 143.8 & & 190.0 & 178.3 & 180.4 & 186.6 & 153.5 & 161.8 & 173.0 & 161.8 & 162.2 & 193.3 & & \\
\hline 104. & $10: 00$ & 39.000 & 180.3 & & 184.7 & & 140.8 & & & 158.6 & 164.7 & 153.4 & 149.9 & 158.0 & 165.9 & 152.1 & 154.3 & 181.7 & & \\
\hline $144 \%$ & $12: 00$ & 39.083 & 170.6 & & 183.8 & & 128.7 & & & 138.2 & 138.6 & 141.1 & 147.2 & 159.7 & 154.5 & 148.5 & 150.7 & 171.9 & & \\
\hline $1,1.1$ & 14:00 & 39.107 & 169.5 & & 188.7 & & 124.1 & & & 136.2 & 135.8 & 128.7 & 154.6 & 158.6 & 156.7 & 161.9 & 159.8 & 158.3 & & \\
\hline $41 \% 1$ & 16:00 & 39.250 & 205.0 & & 194.8 & & 130.1 & & & 179.4 & 164.4 & 161.8 & 171.8 & 175.2 & 177.3 & 182.8 & 174.4 & 164.4 & & \\
\hline 4716 \% & $18: 00$ & 39.333 & 220.9 & & 191.5 & & 139.0 & & 204.4 & 205.2 & 189.7 & 181.2 & 177.4 & 173.6 & 180.8 & 183.2 & 179.8 & 165.6 & & \\
\hline$\% 4 \%$ & $20: 00$ & 39.417 & 214.2 & & 188.2 & & 141.7 & & 209.8 & 202.7 & 193.3 & 184.8 & 164.6 & 168.7 & 186.6 & 171.2 & 174.5 & 153.7 & & \\
\hline $1 \% 1 \%$ & 22:00 & 39.500 & 207.3 & & 186.5 & & 144.4 & & 210.8 & 198.4 & 195.1 & 184.0 & 159.5 & 185.9 & 184.2 & 167.0 & 167.3 & & & \\
\hline $104 \%$ & $0: 00$ & 39.503 & 203.0 & & 184.8 & & 144.4 & & 208.5 & 193.1 & 194.3 & 182.3 & 157.8 & 164.2 & 179.8 & 168.2 & 165.7 & & & \\
\hline $1014 \%$ & $2: 00$ & 39.667 & 203.0 & & 184.8 & & 147.9 & & 205.7 & 190.5 & 193.4 & 180.6 & 157.9 & 185.1 & 179.1 & 165.4 & 164.0 & & & \\
\hline 1014 & $4: 00$ & 39.750 & 200.5 & & 184.0 & & 148.8 & & 205.7 & 187.9 & 195.2 & 180.6 & 157.9 & 162.5 & 179.2 & 165.4 & 164.1 & & & \\
\hline $1 \% 4$ & $6: 00$ & 39.833 & 200.5 & & 185.0 & & 150.6 & & 203.2 & 188.1 & 194.4 & 177.2 & 158.0 & 163.5 & 178.4 & 168.4 & 164.2 & 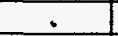 & & \\
\hline $12 \% 1 \%$ & $8: 00$ & 39.917 & 193.6 & & 182.4 & & 148.0 & & 199.8 & 180.8 & 187.5 & 173.7 & 152.8 & 161.7 & 176.7 & 159.4 & 161.6 & & & \\
\hline $1 \%+\%$ & $10: 00$ & $\$ 0.000$ & 175.2 & & 181.4 & & 138.4 & & & 147.2 & 155.2 & 150.0 & 144.8 & 158.1 & 163.5 & 145.3 & 148.4 & & & \\
\hline 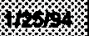 & $12: 00$ & 10.083 & 159.4 & & 187.3 & & 127.0 & & & 125.9 & 130.8 & 124.6 & 142.9 & 158.8 & 148.5 & 145.9 & 152.5 & & & \\
\hline $1 \% \%$ & 14:00 & $\$ 0.167$ & 174.0 & & 193.2 & & 128.0 & & & 147.8 & 142.0 & 139.3 & 172.2 & 162.2 & 157.8 & 172.7 & 163.5 & & & \\
\hline $101 / 1 \%$ & 16:00 & 40.250 & 203.5 & & 195.0 & & 126.9 & & & 178.8 & 182.8 & 165.5 & 171.3 & 176.3 & 175.1 & 182.3 & 172.1 & & & \\
\hline $1018 \%$ & 18:00 & 40.333 & 223.7 & & 193.4 & & 134.8 & & 202.0 & 201.8 & 188.1 & 181.3 & 171.6 & 174.6 & 186.6 & 183.4 & 179.3 & & & \\
\hline $104 \%$ & $20: 00$ & 10.417 & 213.5 & & 187.5 & & 141.0 & & 205.7 & 201.1 & 192.6 & 180.6 & 165.7 & 165.1 & 189.5 & 172.3 & 172.7 & & & \\
\hline 12140 & $22: 00$ & 40.600 & 211.8 & & 187.5 & & 144.5 & & 210.1 & 200.2 & 194.4 & 184.2 & 160.6 & 168.1 & 190.4 & 169.8 & 170.1 & & & \\
\hline 1864 & 0.00 & 20.683 & 206.6 & & 185.8 & & 145.4 & & 209.3 & 195.0 & 194.4 & 180.7 & 158.8 & 186.1 & 183.5 & 186.4 & 168.7 & & & \\
\hline $1 \% 4 \%$ & $2: 00$ & 10.667 & 204.1 & & 185.0 & & 148.0 & & 211.1 & 193.2 & 194.4 & 182.5 & 158.9 & 164.4 & 183.6 & 168.4 & 168.8 & & & \\
\hline $14 \%$ & $4: 00$ & 10.750 & 200.6 & & 185.0 & & 148.8 & & 207.6 & 189.7 & 191.8 & 180.8 & 159.8 & 165.3 & 180.2 & 168.5 & 165.1 & & & \\
\hline $12 \%$ & $6: 00$ & 40.833 & 199.7 & & 185.9 & & 148.1 & & 203.3 & 188.8 & 191.0 & 177.3 & 158.1 & 183.5 & 170.3 & 168.4 & 165.9 & & & \\
\hline $101 \%$ & $8: 00$ & $\$ 0.917$ & 198.0 & & 185.0 & & 149.8 & & 202.4 & 182.7 & 187.5 & 176.4 & 157.2 & 163.5 & 179.3 & 165.6 & 164.3 & & & \\
\hline $121 \% 1$ & $10: 00$ & 41.000 & 190.1 & & 185.8 & & 146.3 & & 190.2 & 178.5 & 179.7 & \begin{tabular}{|l|l|}
165.9 \\
\end{tabular} & 158.0 & 163.5 & 172.3 & 162.0 & 163.3 & & & \\
\hline $1 / 4.4$ & $12: 00$ & 41.083 & 189.2 & & 188.4 & & 143.6 & & 180.6 & 172.8 & 171.8 & 160.6 & 158.8 & 165.2 & 168.6 & 168.3 & 164.1 & & & \\
\hline 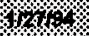 & $0: 00$ & 41.683 & 200.8 & & 187.7 & & 150.7 & & 199.8 & 183.6 & 173.6 & 174.7 & 159.0 & 168.2 & 180.2 & 167.4 & 167.7 & & & \\
\hline 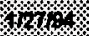 & $12: 00$ & 12.083 & 175.3 & & 188.4 & & 141.1 & & & 143.7 & 144.0 & 142.3 & 152.8 & 162.6 & 162.8 & 155.9 & 160.7 & & & \\
\hline $121 \%$ & 0.00 & 42.583 & 207.6 & & 491.2 & & 154.1 & & 207.7 & 194.2 & 171.1 & 187.8 & 162.5 & 167.1 & 183.7 & 170.0 & 167.7 & & & \\
\hline $14=1$ & $12: 00$ & 43.083 & 183.7 & & 189.5 & & 158.5 & & 189.5 & 173.8 & 154.8 & 171.3 & 158.2 & 165.4 & 178.0 & 162.2 & 163.5 & & & \\
\hline $12 \%$ & $0_{i}$ & 43.593 & 203.5 & & 189.6 & & 165.6 & & 202.7 & 183.8 & 173.8 & 177.5 & 159.3 & 167.3 & 185.7 & 170.3 & 168.8 & & & \\
\hline 15. & $12: 00$ & 4.083 & 170.3 & & 194.8 & & 150.0 & & & 133.4 & 131.2 & 137.3 & 151.4 & 184.7 & 162.3 & 149.3 & 157.6 & & & \\
\hline 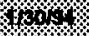 & $0: 00$ & 4.683 & 208.1 & & 193.1 & & 160.4 & & & 188.2 & 177.3 & 177.8 & 168.8 & 970.8 & 184.0 & 172.0 & 170.8 & & & \\
\hline 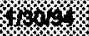 & $12: 00$ & 46.083 & 184.3 & & 191.4 & & 160.4 & & & 167.0 & 151.3 & 161.8 & 155.8 & 185.6 & 172.8 & 159.8 & 161.1 & & & \\
\hline $151 \%$ & $0: 00$ & 46.693 & 208.8 & & 192.4 & & 174.4 & & & 191.0 & 173.1 & 180.8 & 163.8 & 171.0 & 189.4 & 171.4 & 172.6 & & & \\
\hline 45 & $12: 00$ & 40.093 & 183.5 & & 195.0 & & 165.7 & & & 149.4 & 147.9 & 159.2 & 158.8 & 169.2 & 172.0 & 160.8 & 181.3 & & & \\
\hline 2664 & $0: 00$ & 46.683 & 215.8 & & 193.4 & & 222.1 & & 185.6 & 198.4 & 176.7 & 190.1 & 169.2 & 171.1 & & 175.8 & 174.5 & & & \\
\hline
\end{tabular}


Table 5. Tensiometer Data

\begin{tabular}{|c|c|c|c|c|c|c|c|c|c|c|c|c|c|c|c|c|c|c|c|c|}
\hline & & & & & & & & & Ten & siometer & Data & & & & & & & & & \\
\hline & & Test & $\begin{array}{c}\text { Soll } \\
\text { Moisture } \\
\text { Tension } \\
\left(\mathrm{Cm} \mathrm{H}_{2} \mathrm{O}\right)\end{array}$ & $\begin{array}{c}\text { Soll } \\
\text { Molsture } \\
\text { Tenston } \\
\left(\mathrm{Cm} \mathrm{H}_{2} \mathrm{O}\right)\end{array}$ & $\begin{array}{c}\text { Soil } \\
\text { Moisture } \\
\text { Tension } \\
\left(\mathrm{Cm} \mathrm{H}_{2} \mathrm{O}\right)\end{array}$ & $\begin{array}{c}\text { Soll } \\
\text { Moisture } \\
\text { Tension } \\
\left(\mathrm{Cm} \mathrm{H}_{2} \mathrm{O}\right) \\
\end{array}$ & $\begin{array}{c}\text { Soll } \\
\text { Molsture } \\
\text { Tenslon } \\
\left(\mathrm{Cm} \mathrm{H}_{2} \mathrm{O}\right) \\
\end{array}$ & $\begin{array}{c}\text { Soll } \\
\text { Moisture } \\
\text { Tenslon } \\
\left(\mathrm{cm} \mathrm{H}_{2} \mathrm{O}\right)\end{array}$ & $\begin{array}{c}\text { Soll } \\
\text { Molsture } \\
\text { Tension } \\
\left(\mathrm{Cm}_{2} \mathrm{O}\right)\end{array}$ & $\begin{array}{c}\text { Soil } \\
\text { Moisture } \\
\text { Tension } \\
\left(\mathrm{em}^{\mathrm{H}} \mathrm{O} \mathrm{O}\right)\end{array}$ & $\begin{array}{c}\text { Soll } \\
\text { Molsture } \\
\text { Tonsion } \\
\left(\mathrm{cm} \mathrm{H}_{2} \mathrm{O}\right)\end{array}$ & $\begin{array}{c}\text { Soll } \\
\text { Molsture } \\
\text { Tension } \\
\left(\mathrm{cm} \mathrm{H}_{2} \mathrm{O}\right)\end{array}$ & $\begin{array}{c}\text { Soll } \\
\text { Molsture } \\
\text { Tension } \\
\left.\text { (cm } \mathrm{H}_{2} \mathrm{O}\right)\end{array}$ & $\begin{array}{c}\text { Soll } \\
\text { Moisture } \\
\text { Tenstion } \\
\left(\mathrm{cm} \mathrm{H}_{2} \mathrm{O}\right) \\
\end{array}$ & $\begin{array}{c}\text { Soll } \\
\text { Moleture } \\
\text { Tonston } \\
\left(\mathrm{cm} \mathrm{H}_{2} \mathrm{O}\right) \\
\end{array}$ & 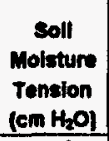 & $\begin{array}{c}\text { Soll } \\
\text { Moisture } \\
\text { Tension } \\
\left(\mathrm{cm} \mathrm{H}_{2} \mathrm{O}\right)\end{array}$ & $\begin{array}{c}\text { Soll } \\
\text { Moisture } \\
\text { Tension } \\
\left(\mathrm{Cm} \mathrm{H}_{2} \mathrm{O}\right)\end{array}$ & $\begin{array}{c}\text { Soll } \\
\text { Molsture } \\
\text { Tension } \\
\left(\mathrm{Cm}_{2} \mathrm{H}_{2}\right) \\
\end{array}$ & $\begin{array}{c}\text { Soll } \\
\text { Moisture } \\
\text { Tension } \\
\text { (cm Hz) }\end{array}$ \\
\hline Date & Time & Days' & $C 1^{\prime \prime}$ & $B 1^{\prime \prime}$ & $A 1^{\prime \prime}$ & $C 2^{b}$ & $B 2^{6}$ & $A 2^{b}$ & $C 3^{\mathrm{C}}$ & $\mathrm{B3}^{\mathrm{C}}$ & $\mathrm{A3}^{\mathrm{C}}$ & $c 4^{d}$ & $84^{d}$ & $A A^{d}$ & $\mathrm{C5}^{\circ}$ & $B 6^{\circ}$ & $A 5^{\circ}$ & $\mathrm{Co}^{\prime}$ & $B 6^{\prime}$ & $A 6^{\prime}$ \\
\hline 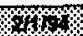 & $12: 00$ & 47.083 & 180.9 & & 203.8 & & 162.3 & & & 138.8 & 204.5 & 155.0 & 155.1 & 167.5 & 213.7 & 187.1 & 173.4 & & & \\
\hline 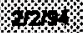 & $0: 00$ & 47.683 & 216.8 & & 198.0 & & 311.4 & & 198.7 & 200.9 & 197.6 & 192.7 & 170.0 & 173.7 & & 175.9 & 177.1 & & & \\
\hline $4 \%$ & $12: 00$ & 48.093 & 181.8 & & 202.8 & & 160.5 & & & 145.0 & 217.5 & 151.5 & 161.2 & 168.4 & & 179.2 & 178.9 & & & \\
\hline $24 \%$ & $0: 00$ & 40.583 & 215.9 & & 196.9 & & 184.9 & & 198.7 & 195.5 & 186.3 & 190.1 & 170.0 & 174.6 & & 174.1 & 178.8 & & & \\
\hline 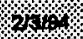 & $12: 00$ & 49.083 & 171.3 & & 199.3 & & 150.9 & & & 125.5 & 108.7 & 139.1 & 152.3 & 169.2 & & 142.4 & 158.6 & & & \\
\hline 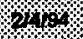 & $0: 00$ & 49.683 & 216.6 & & 201.8 & & 163.1 & & 181.8 & 201.6 & & 189.8 & 172.4 & 178.8 & & 173.8 & 179.4 & & & \\
\hline 24.4 & $12: 00$ & 50.083 & 193.0 & & 204.5 & & 154.3 & & 161.8 & 167.9 & & 163.6 & 169.7 & 177.9 & & 166.8 & 174.1 & & & \\
\hline $24.34 \%$ & 0.00 & 50.683 & 218.4 & & 202.9 & & 180.5 & & & 198.9 & & 182.8 & 175.8 & 182.4 & & 178.3 & 182.0 & & & \\
\hline 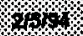 & $12: 00$ & 51.083 & 170.3 & & 198.4 & & 148.6 & & & 129.0 & & 140.8 & 144.5 & 168.3 & & 124.0 & 150.7 & & & \\
\hline $136 \%$ & $0: 00$ & 51.683 & 223.0 & & 201.3 & & 170.2 & & & 208.9 & & 203.3 & 178.8 & 181.7 & & 184.7 & 185.8 & & & \\
\hline $28 \%$ & $12: 00$ & 52.083 & 176.6 & & 202.0 & & 151.8 & & & 131.7 & & 148.8 & 149.0 & 171.9 & & 135.5 & 159.5 & & & \\
\hline $2 \pi$ & $0: 00$ & 62.583 & 224.7 & & 202.1 & & 165.9 & & & 208.9 & & 198.9 & 176.1 & 182.5 & & 183.8 & 185.7 & $\because$ & & \\
\hline $2 \pi+2$. & $12: 00$ & 53.083 & 198.4 & & 203.8 & & 157.1 & & & 169.8 & & 168.1 & 168.2 & 178.9 & & 158.3 & 171.7 & & & \\
\hline /2.15. & $0: 00$ & 63.683 & 217.7 & & 207.4 & & 158.0 & & & 192.8 & & 188.3 & 178.7 & 182.5 & & 178.8 & 183.1 & & & \\
\hline 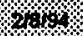 & $12: 00$ & 54.083 & 208.9 & & 200.5 & & 154.5 & & & 182.2 & & 181.3 & 172.6 & 183.4 & & 168.8 & 178.7 & & & \\
\hline $2 \% 1 \%$ & $0: 00$ & 64.683 & 220.3 & & 206.5 & & 163.3 & & & 195.6 & & 192.7 & 179.7 & 188.1 & & 178.6 & 184.9 & & & \\
\hline 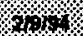 & $12: 00$ & 56.083 & 210.8 & & 220.5 & & 982.3 & & & 180.5 & & 177.8 & 188.5 & 192.1 & & 173.2 & 185.6 & & & \\
\hline $240 \%$ & 0.00 & 65.683 & 229.2 & & 205.8 & & 172.8 & & & 212.5 & & 206.0 & 185.9 & 187.0 & & 190.1 & 191.1 & & & \\
\hline $3.10 \%$ & $12: 00$ & 56.083 & 177.5 & & 208.2 & & & & & 130.0 & & 148.8 & 149.8 & 175.5 & & 123.3 & 160.5 & & & \\
\hline 3r16\% & $0: 00$ & 66.683 & 220.8 & & 207.4 & & 167.6 & & & 213.3 & & 200.8 & 184.9 & 187.8 & & 180.0 & 191.9 & & & \\
\hline 22616 & $12: 00$ & 57.083 & 175.7 & & 206.4 & & 148.4 & & & 142.4 & & 161.1 & 150.7 & $175: 4$ & & 117.1 & 159.6 & & & \\
\hline 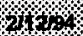 & $0: 00$ & 57.683 & 233.5 & & 209.2 & & 169.4 & & & 210.7 & & 203.3 & 187.6 & 189.6 & & 193.5 & 194.5 & & & \\
\hline 2134 & $12: 00$ & 58.083 & 195.1 & & 211.0 & & 169.4 & & & 161.1 & & 171.7 & 160.5 & 182.6 & & 133.1 & 169.3 & & & \\
\hline 2414 & 0.00 & 50.683 & 238.0 & & 208.5 & & 192.9 & & & 223.2 & & 217.4 & 180.4 & 182.3 & & 197.2 & 199.1 & & & \\
\hline $217 \%$ & $12: 00$ & 59.083 & 178.5 & & 209.2 & & $\$ 74.5$ & & & 124.7 & & 149.8 & 144.8 & 178.2 & & & 159.8 & & & \\
\hline 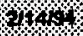 & $0: 00$ & 60.683 & 234.4 & & 209.3 & & 184.2 & & & 214.2 & & 205.9 & 186.8 & 189.6 & & 100.1 & 193.7 & & & \\
\hline 124.4 & $12: 00$ & 60.083 & 174.0 & & 205.6 & & 162.4 & & & 117,6 & & 144.5 & 141.2 & 175.5 & & & 155.3 & & & \\
\hline 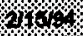 & $0: 00$ & 00.583 & 240.5 & & 211.9 & & 181.6 & & & 220.5 & & 212.1 & 194.6 & 194.0 & & 193.6 & 199.0 & & & \\
\hline 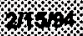 & $12: 00$ & 61.093 & 174.0 & & 209.1 & & & & & 118.5 & & 144.5 & 141.2 & 179.0 & & & 157.8 & & & \\
\hline 2116. & $0: 00$ & 61.683 & 244.8 & & 211.9 & & 184.1 & & & 230.2 & 172.4 & 218.2 & 197.2 & 184.8 & & 198.8 & 202.3 & & & \\
\hline (12 & $12: 00$ & 62.083 & 165.2 & & 209.9 & & & & & 130.8 & 142.7 & 139.2 & 142.0 & 178.0 & & & 157.8 & & & \\
\hline $477_{1}$ & 0.00 & 62.583 & 242.0 & & 214.3 & & 174.4 & & & 218.4 & 204.5 & 209.2 & 202.1 & 198.2 & 192.1 & 180.6 & 201.2 & & & \\
\hline 4114 & $12: 00$ & 63.093 & 172.1 & & 202.8 & & & & & 140.5 & 167.0 & 149.8 & 143.6 & 177.1 & 163.3 & & 158.6 & & & \\
\hline 82161. & 0.00 & 63.583 & 234.9 & & 215.1 & & & & & 208.0 & 204.4 & 196.8 & 197.7 & 199.8 & 188.3 & 178.3 & 185.0 & & & \\
\hline 32 & $12: 00$ & 04.083 & 210.6 & & 211.6 & & & & & 187.5 & 191.4 & 184.7 & 181.2 & 193.0 & 185.9 & & 180.4 & & & \\
\hline 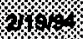 & $0: 00$ & 04.683 & 243.9 & & 211.8 & & & & & 218.5 & 220.3 & 216.4 & 199.8 & 200.2 & 214.0 & 187.4 & 203.2 & & & \\
\hline 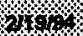 & $12: 00$ & 66.093 & 180.6 & & 216.2 & & & & & 163.7 & 188.3 & 162.1 & 158.7 & 188.9 & 180.8 & & 168.4 & & & \\
\hline 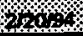 & $0: 00$ & 65.603 & 244.0 & & 214.5 & & 180.7 & & & 219.8 & 223.8 & 215.6 & 200.7 & 201.1 & 217.5 & 183.1 & 203.3 & & & \\
\hline
\end{tabular}


Table 5. Tensiometer Data

\begin{tabular}{|c|c|c|c|c|c|c|c|c|c|c|c|c|c|c|c|c|c|c|c|c|}
\hline & & & & & & & & & Ten: & siometer & Data & & & & & & & & & \\
\hline & & Test & $\begin{array}{c}\text { Soll } \\
\text { Molsture } \\
\text { Tension } \\
\left(\mathrm{Cm} \mathrm{H}_{2} \mathrm{O}\right) \\
\end{array}$ & 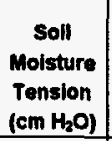 & $\begin{array}{c}\text { Soll } \\
\text { Moisture } \\
\text { Tenslon } \\
\left(\mathrm{C} \mathrm{H}_{2} \mathrm{O}\right) \\
\end{array}$ & 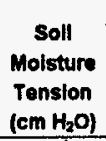 & 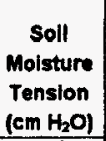 & 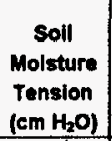 & $\begin{array}{c}\text { Soil } \\
\text { Molsture } \\
\text { Tension } \\
\left(\mathrm{cm} \mathrm{H}_{2} \mathrm{O}\right)\end{array}$ & $\begin{array}{c}\text { Solt } \\
\text { Moisture } \\
\text { Tension } \\
\left(\mathrm{Cm} \mathrm{H}_{2} \mathrm{O}\right)\end{array}$ & $\begin{array}{c}\text { Soll } \\
\text { Molsture } \\
\text { Tension } \\
\left.\text { (cm } \mathrm{H}_{2} \mathrm{O}\right) \\
\end{array}$ & $\begin{array}{c}\text { Soll } \\
\text { Molsture } \\
\text { Tension } \\
\left(\mathrm{Cm} \mathrm{H}_{2} \mathrm{O}\right) \\
\end{array}$ & $\begin{array}{c}\text { Soil } \\
\text { Moisture } \\
\text { Tension } \\
\left(\mathrm{Cm}_{2} \mathrm{H}_{2} \mathrm{O}\right) \\
\end{array}$ & $\begin{array}{c}\text { Soll } \\
\text { Moisture } \\
\text { Tension } \\
\left(\mathrm{em} \mathrm{H}_{2} \mathrm{O}\right) \\
\end{array}$ & $\begin{array}{c}\text { Soll } \\
\text { Moisture } \\
\text { Tenston } \\
\left(\mathrm{Cm} \mathrm{H}_{2} \mathrm{O}\right) \\
\end{array}$ & $\begin{array}{c}\text { Soll } \\
\text { Moisture } \\
\text { Tension } \\
\left(\mathrm{C} \mathrm{H} \mathrm{H}_{2} \mathrm{O}\right) \\
\end{array}$ & $\begin{array}{c}\text { Soll } \\
\text { Molsture } \\
\text { Tension } \\
\left(\mathrm{Cm} \mathrm{H} \mathrm{H}_{2} \mathrm{O}\right) \\
\end{array}$ & $\begin{array}{c}\text { Soll } \\
\text { Molsture } \\
\text { Tenaion } \\
\left(\mathrm{Cm} \mathrm{H}_{2} \mathrm{O}\right)\end{array}$ & $\begin{array}{c}\text { Soll } \\
\text { Molsture } \\
\text { Tension } \\
\left(\mathrm{Cm} \mathrm{H}_{2} \mathrm{O}\right) \\
\end{array}$ & $\begin{array}{c}\text { Soll } \\
\text { Molature } \\
\text { Tension } \\
\left.\text { (cm } \mathrm{H}_{2} \mathrm{O}\right)\end{array}$ \\
\hline Date & Time & Days' & $C 1^{\prime \prime}$ & $B 1^{\prime \prime}$ & $A 1^{\circ}$ & $\mathrm{C2}^{\mathrm{b}}$ & $\mathbf{B 2}^{\mathrm{b}}$ & $\mathbf{A 2}^{6}$ & $\mathrm{Cs}^{\mathrm{c}}$ & $\mathrm{B3}^{\mathrm{c}}$ & $\mathbf{A 3}^{\mathrm{C}}$ & $C 4^{\mathrm{d}}$ & $\mathrm{B4}^{\mathrm{d}}$ & $\mathbf{A} 4^{\mathrm{d}}$ & $\mathrm{C5}^{\circ}$ & $85^{\circ}$ & $\mathrm{A5}^{\circ}$ & $\mathrm{co}^{\prime}$ & $86^{\prime}$ & $A G^{\prime}$ \\
\hline \%on & $12: 00$ & 66.083 & 188.0 & & 217.0 & & & & & 151.3 & 183.7 & 159.4 & 150.8 & 186.9 & 180.8 & & 167.5 & & & \\
\hline 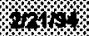 & $0: 00$ & 66.583 & 247.5 & & 214.5 & & 184.2 & & & 223.1 & 224.7 & 218.1 & 204.2 & 202.0 & 217.6 & & 204.1 & & & \\
\hline $4 \times 14$ & $12 ;$ & 67.083 & 214.2 & & 214.4 & & 174.5 & & & 184.9 & 202.8 & 184.0 & 179.8 & 194.8 & 198.3 & & 182.3 & & & \\
\hline 3224 & $0: 00$ & 67.583 & 237.0 & & 216.3 & & 178.1 & & & 208.0 & 215.9 & 202.4 & 198.9 & 201.0 & 209.7 & & 196.3 & & & \\
\hline $2 \% 1$ & $12: 00$ & 68.083 & 222.1 & & 222.3 & & 172.0 & & & 191.2 & 204.6 & 191.8 & 189.3 & 204.5 & 189.2 & & 189.3 & & & \\
\hline 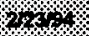 & $0: 00$ & 60.563 & 244.1 & & 215.5 & & 188.0 & & & 214.3 & 224.7 & 213.9 & 199.1 & 200.3 & 217.7 & & 201.7 & & & \\
\hline $828 \%$ & $12: 00$ & $\infty 9.083$ & 180.7 & & 214.5 & & 174.6 & & & 146.9 & 184.6 & 187.4 & 148.3 & 187.9 & 184.5 & & 185.0 & & & \\
\hline 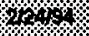 & $0: 00$ & $\infty 0.583$ & 253.7 & & 218.1 & & 182.8 & & & 224.1 & 230.0 & 221.8 & 213.9 & 204.7 & 221.2 & & 210.4 & & & \\
\hline $20 \%$ & $12: 00$ & 70.083 & 174.9 & & 215.3 & & & & & & 168.0 & 142.8 & 135.1 & 184.3 & 171.3 & & 154.5 & & & \\
\hline $820-1$ & $0: 00$ & 70.583 & 258.8 & & 220.6 & & 185.8 & & & 226.6 & 228.4 & 240.1 & 219.8 & 208,1 & 223.6 & & 211.0 & & & \\
\hline $1,4.4 \%$ & $12: 00$ & 71.083 & 167.8 & & 216.8 & & 165.7 & & & & 160.9 & 153.2 & 130.8 & 182.4 & 171.1 & & 153.4 & & & \\
\hline $2 \% 4$ & $0: 00$ & 71.583 & 265.7 & & 221.4 & & 188.4 & & & 237.2 & 228.8 & 238.5 & 225.8 & 211.5 & 227.8 & & 218.7 &. & & \\
\hline $2 \%-4$ & $12: 00$ & 72.093 & 173.1 & & 214.3 & & 168.3 & & & & 165.3 & 155.0 & 131.5 & 182.5 & 174.7 & & 156.1 & & & \\
\hline 3004 & $0: 00$ & 72.583 & 262.1 & & 222.2 & & 183.1 & & & 231.8 & 225.4 & 229.4 & 228.3 & 213.2 & 225.0 & & 215.1 & & & \\
\hline 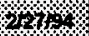 & $12: 00$ & 73.083 & 203.5 & & 220.3 & & 169.9 & & & 161.8 & 787.0 & 175.9 & 172.3 & 198.2 & 195.3 & & 177.6 & & & \\
\hline $21 \%$ & 0.00 & 73.683 & 254.2 & & 222.2 & & 177.8 & & & 217.6 & 219.3 & 218.9 & 219.6 & 212.3 & 219.8 & & 209.9 & & & \\
\hline $170 \%$ & $12: 00$ & 74.093 & 223.7 & & 223.9 & & 173.5 & & & 183.1 & 201.0 & 192.6 & 189.0 & 205.3 & 205.9 & & 189.9 & & & \\
\hline 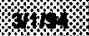 & $0: 00$ & 74.503 & 256.1 & & 223.2 & & 190.2 & & & 220.4 & 227.2 & 223.4 & 214.6 & 212.5 & 225.3 & & 210.1 & & & \\
\hline 34 & $12: 00$ & 76.083 & 184.4 & & 216.8 & & 175.3 & & & & & 160.2 & 133.3 & 187.7 & 181.7 & & 165.7 & & & \\
\hline 3114 & 0.00 & 75.583 & 268.3 & & 224.1 & & 196.2 & & & 233.7 & 230.7 & 235.7 & 230.2 & 216.0 & 233.0 & & 221.3 & & & \\
\hline 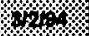 & $12: 00$ & 76.083 & 173.1 & & 217.8 & & 177.8 & & & & & 153.2 & 135.9 & 187.7 & 175.5 & & 161.3 & & & \\
\hline 4.10 & $0: 00$ & 76.583 & 267.4 & & 224.0 & & 194.4 & & & 232.7 & 219.3 & 231.2 & 233.6 & 215.9 & 229.5 & & 218.6 & & & \\
\hline 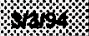 & $12: 00$ & 77.083 & 162.5 & & 214.2 & & 173.4 & & & & & 148.1 & 128.8 & 184.1 & 168.5 & & 155.8 & & & \\
\hline (1) & $0: 00$ & 77.583 & 270.9 & & 224.8 & & 191.8 & & & 234.5 & 181.1 & 233.0 & 234.5 & 217.7 & 230.4 & & 218.7 & & & \\
\hline 414 & $12: 00$ & 78.083 & 165.1 & & 215.9 & & & & & & & 140.9 & 118.6 & 181.5 & 168.7 & & 154.2 & & & \\
\hline (6) & 0.00 & 78.583 & 274.4 & & 228.7 & & 184.8 & & & 238.1 & & 235.6 & 247.6 & 222.1 & 232.1 & & 223.0 & & & \\
\hline 444 & $12: 00$ & 79.083 & 172.1 & & 216.7 & & & & & & & 144.4 & 128.6 & 184.1 & 170.1 & & 158.8 & & & \\
\hline $4 \%$ & $0: 00$ & 79.683 & 276.9 & & 228.4 & & & & & 239.8 & & 235.8 & 247.5 & 222.1 & 233.8 & & 222.1 & & & \\
\hline 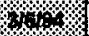 & $12: 00$ & 80.083 & 168.8 & & 215.0 & & & & & & & 144.4 & 118.3 & 183.2 & & & 157.6 & & & \\
\hline ryo & $0: 00$ & 80.693 & 282.1 & & 230.1 & & & & & 224.7 & & 240.8 & 250.8 & 225.5 & & & 220.3 & & & \\
\hline 11194 & $12: 00$ & 81.083 & 234.9 & & 228.2 & & & & & & & 204.0 & 202.0 & 210.5 & & & 195.8 & & & \\
\hline \% & $0: 00$ & 81.693 & 261.3 & & 228.4 & & & & & & & 225.1 & 220.6 & 218.6 & & & 211.7 & & & \\
\hline (18) & $12: 00$ & 82.083 & 247.4 & & 226.7 & & & & & & & 221.7 & 211.1 & 209.8 & & & 205.8 & & & \\
\hline $2 \%$ & $0: 00$ & 82.693 & 262.3 & & 230.3 & & & & & 123.9 & & 228.7 & 207.7 & 217.8 & & & 210.2 & & & \\
\hline 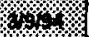 & $12: 00$ & 83.083 & 224.7 & & 225.8 & & & & & & & 198.9 & 184.9 & 205.4 & & & 191.0 & & & \\
\hline $1010 \%$ & $0: 00$ & 83.693 & 278.9 & & 232.8 & & 178.1 & & & 121.2 & & 241.9 & 234.7 & 223.0 & & & 223.2 & & & \\
\hline $3 \%$ & $12: 00$ & 84.083 & 183.6 & & 224.8 & & & & & & & 158.5 & 129.0 & 186.5 & & & 170.0 & & & \\
\hline 1) & $0: 00$ & 84.683 & 273.5 & & 233.7 & & 171.0 & & & & & 230.4 & 233.7 & 221.2 & & & 218.7 & & & \\
\hline
\end{tabular}


Table 5. Tensiometer Data

\begin{tabular}{|c|c|c|c|c|c|c|c|c|c|c|c|c|c|c|c|c|c|c|c|c|}
\hline & & & & & & & & & Ten & siometer & Data & & & & & & & & & \\
\hline & & Test & $\begin{array}{c}\text { Soll } \\
\text { Moisture } \\
\text { Tension } \\
\left(\mathrm{Cm} \mathrm{H} \mathrm{H}_{2} \mathrm{O}\right) \\
\end{array}$ & 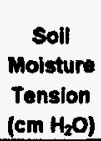 & $\begin{array}{c}\text { Soll } \\
\text { Moisture } \\
\text { Tension } \\
\left(\mathrm{em} \mathrm{H}_{2} \mathrm{O}\right) \\
\end{array}$ & $\begin{array}{c}\text { Soll } \\
\text { Molsture } \\
\text { Tension } \\
\left(\mathrm{Cm} \mathrm{H}_{2} \mathrm{O}\right) \\
\end{array}$ & $\begin{array}{c}\text { Soll } \\
\text { Moisture } \\
\text { Tension } \\
\text { (cm } \mathrm{H}_{2} \mathrm{O} \text { ) } \\
\end{array}$ & $\begin{array}{c}\text { Soil } \\
\text { Moisture } \\
\text { Tension } \\
\left(\mathrm{em} \mathrm{H}_{2} \mathrm{O}\right) \\
\end{array}$ & $\begin{array}{c}\text { Soll } \\
\text { Moisture } \\
\text { Tension } \\
\left(\mathrm{Cm} \mathrm{H}_{2} \mathrm{O}\right) \\
\end{array}$ & $\begin{array}{c}\text { Soll } \\
\text { Molsture } \\
\text { Tension } \\
\left(\mathrm{Cm} \mathrm{H}_{2} \mathrm{O}\right) \\
\end{array}$ & $\begin{array}{c}\text { Soll } \\
\text { Moisture } \\
\text { Tension } \\
\left(\mathrm{Cm} \mathrm{H}_{2} \mathrm{O}\right) \\
\end{array}$ & 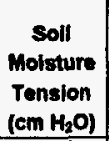 & $\begin{array}{c}\text { Soil } \\
\text { Moisture } \\
\text { Tenslon } \\
\left(\mathrm{Cm} \mathrm{H}_{2} \mathrm{O}\right) \\
\end{array}$ & $\begin{array}{c}\text { Soll } \\
\text { Molisture } \\
\text { Tension } \\
\left(\mathrm{Cm} \mathrm{H}_{2} \mathrm{O}\right) \\
\end{array}$ & $\begin{array}{c}\text { Soll } \\
\text { Molsture } \\
\text { Tension } \\
\left(\mathrm{Cm} \mathrm{H}_{2} \mathrm{O}\right) \\
\end{array}$ & $\begin{array}{c}\text { Soll } \\
\text { Moisture } \\
\text { Tenslon } \\
\text { (em H2O) }\end{array}$ & $\begin{array}{c}\text { Soll } \\
\text { Molsture } \\
\text { Tension } \\
\left(\mathrm{em} \mathrm{H}_{2} \mathrm{O}\right) \\
\end{array}$ & $\begin{array}{c}\text { Soll } \\
\text { Molsture } \\
\text { Tonsion } \\
\left.\text { (cm } \mathrm{H}_{2} \mathrm{O}\right)\end{array}$ & $\begin{array}{c}\text { Soll } \\
\text { Molsture } \\
\text { Tenslon } \\
\left.\text { (em } \mathrm{H}_{2} \mathrm{O}\right) \\
\end{array}$ & $\begin{array}{c}\text { soll } \\
\text { Molature } \\
\text { Tenslon } \\
\left(\mathrm{Cm} \mathrm{H} \mathrm{H}_{2} \mathrm{O}\right) \\
\end{array}$ \\
\hline Date & Time & Days' & $C 1^{\circ}$ & $B 1^{\circ}$ & $A 1^{2}$ & $\mathrm{C2}^{\mathrm{b}}$ & $\mathrm{B2}^{\mathrm{b}}$ & $A 2^{6}$ & $\mathrm{C3}^{\mathrm{c}}$ & $\mathrm{B3}^{\mathrm{C}}$ & $\mathrm{A}^{\mathrm{C}}$ & $\mathrm{CA}^{\mathrm{d}}$ & $\mathrm{B4}^{\mathrm{d}}$ & $A 4^{\mathrm{d}}$ & $\mathrm{cos}^{\circ}$ & $\mathrm{B} 5^{\circ}$ & $\mathbf{A 5}^{\circ}$ & $C 6^{\prime}$ & $86^{t}$ & $A 6^{f}$ \\
\hline 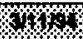 & 12:00 & 85.083 & 185.2 & & 228.4 & & & & & & & 156.8 & 158.5 & 195.5 & & & 171.6 & & & \\
\hline 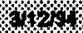 & $0: 00$ & 85.683 & 269.1 & & 235.4 & & 167.5 & & & & & 228.6 & 228.4 & 222.0 & & & 217.8 & & & \\
\hline 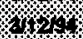 & $12: 00$ & 86.083 & 250.0 & & 231.1 & & 175.4 & & & & & 217.3 & 217.2 & 213.3 & & & 207.5 & & & \\
\hline 4 4. & $0: 00$ & 86.683 & 275.4 & & 233.8 & & 190.2 & & & & & 240.9 & 224.2 & 222.1 & & & 220.6 & & & \\
\hline (1) & $12: 00$ & 87.083 & 184.0 & & 231.8 & & 177.0 & & & & & 171.6 & 157.7 & 200.0 & & & 172.8 & & & \\
\hline 13\% & $0: 00$ & 87.683 & 284.0 & & 237.2 & & 188.4 & & & & & 241.8 & 230.2 & 226.5 & 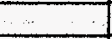 & & 224.8 & & & \\
\hline 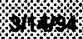 & $12: 00$ & 88.083 & 972.2 & & 223.0 & & 165.7 & & & & & 142.6 & 116.7 & 187.6 & & & 163.0 & & & \\
\hline 4 & $0: 00$ & 88.683 & 286.6 & & 238.9 & & 177.9 & & & & & 238.9 & 251.1 & 231.7 & & & 225.6 & & & \\
\hline 4 & $12: 00$ & 89.083 & 171.2 & & 221.1 & & 165.5 & & & & & 146.0 & 132.2 & 189.3 & & & 186.2 & & & \\
\hline 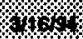 & $0: 00$ & 89.583 & 296.0 & & 240.5 & & 176.1 & & & & & 246.7 & 250.8 & 236.7 & & & 230.8 & & & \\
\hline Xit. & $12 ; 00$ & 90.083 & 177.3 & & 223.8 & & 163.9 & & & & & 152.1 & 124.4 & 196.3 & & & 180.2 & & & \\
\hline X & $0: 00$ & $\infty 0.683$ & 293.4 & & 237.0 & & 169.2 & & & & & 244.1 & 257.8 & 236.7 & & & 227.1 & & & \\
\hline 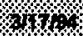 & $12: 00$ & 91.083 & 183.0 & & 215.9 & & & & & & & 168.1 & 154.0 & 185.4 & & & 174.1 & & & \\
\hline 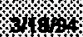 & $0: 00$ & 91.683 & 293.5 & & 235.3 & & & & & & & 245.9 & 251.8 & 236.8 & & & 229.0 & & & \\
\hline 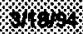 & $12: 00$ & 92.083 & 182.5 & & 217.5 & & & & & & & 156.4 & 139.1 & 194.4 & & & 180.0 & & & \\
\hline \% & $0: 00$ & 92.683 & 287.3 & & 235.3 & & & & & & & 240.6 & 252.7 & 237.6 & & & 228.9 & & & \\
\hline 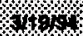 & $12: 00$ & 93.083 & 210.5 & & 214.2 & & & & & & & 182.8 & 155.0 & 199.8 & & & 185.4 & & & \\
\hline 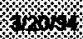 & $0: 00$ & 93.693 & 288.4 & & 234.4 & & 170.9 & & & & & 238.8 & 247.4 & 237.6 & & & 225.4 & & & \\
\hline 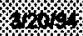 & $12: 00$ & 94.083 & 242.8 & & 228.3 & & 167.5 & & & & & 208.2 & 198.7 & 213.9 & & & 201.2 & & & \\
\hline 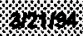 & $0: 00$ & 94.683 & 293.8 & & 235.5 & & 181.5 & & & & & 249.4 & 243.3 & 237.7 & & & 229.1 & & & \\
\hline s & $12: 00$ & 96.083 & 190.5 & & 223.0 & & 169.1 & & & & & 158.2 & 122.8 & 198.0 & & & 178.5 & & & \\
\hline , & $0: 00$ & 96.683 & 300.5 & & 235.4 & & 177.8 & & & & & 249.4 & 258.0 & 240.2 & & & 228.0 & & & \\
\hline 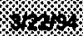 & $12: 00$ & 96.083 & 211.3 & & 236.0 & & 181.2 & & & & & 175.7 & 166.2 & 209.3 & & & 181.9 & & & \\
\hline 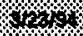 & $0: 00$ & 90.683 & 299.5 & & 236.2 & & 166.8 & & & & & 244.9 & 251.8 & 241.8 & & & 228.9 & & & \\
\hline 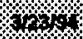 & $12: 00$ & 97.083 & 199.0 & & 222.8 & & & & & & & 163.4 & 140.8 & 201.4 & & & 188.7 & & & \\
\hline 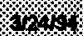 & $0: 00$ & 97.683 & 302.9 & & 238.7 & & 170.0 & & & & & 249.1 & 257.7 & 245.3 & & & 232.2 & & & \\
\hline 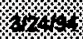 & $12: 00$ & 98.083 & 209.5 & & 222.8 & & 165.5 & & & & & 177.3 & 194.8 & 204.9 & & & 195.7 & & & \\
\hline$\sqrt{2}<3$ & $0: 00$ & 88.683 & 308.1 & & 237.8 & & 171.7 & & & & & 251.7 & 247.2 & 241.7 & & & 231.3 & & & \\
\hline 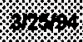 & $12: 00$ & 99.083 & 243.4 & & 229.7 & & 162.9 & & & & & 198.2 & 175.6 & 212.7 & & & 190.4 & & & \\
\hline$x$ & $0: 00$ & 89.683 & 299.3 & & 238.6 & & 168.2 & & & & & 247.3 & 240.2 & 239.8 & & & 226.9 & & & \\
\hline 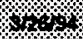 & $12: 00$ & 100.083 & 234.8 & & 208.0 & & & & & & & 190.4 & 151.4 & 197.8 & & & 179.3 & & & \\
\hline 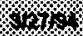 & $0: 00$ & 100.583 & 288.6 & & 240.5 & & 178.1 & & & & & 247.4 & 246.5 & 240.0 & & & 228.0 & & & \\
\hline $2 \%$ & $12: 00$ & 101.083 & 283.8 & & 247.5 & & 183.0 & & & & & 229.9 & 230.0 & 234.8 & & & 219.3 & & & \\
\hline 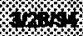 & $0: 00$ & 101.563 & 300.6 & & 239.0 & & 197.8 & & & & & 255.4 & 237.2 & 234.9 & & & 232.6 & & & \\
\hline 8.4 & $12: 00$ & 102.083 & 235.0 & & 228.6 & & 183.1 & & & & & 188.2 & 138.8 & 204.1 & & & 183.0 & & & \\
\hline 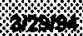 & $0: 00$ & 102.583 & 308.6 & & 242.4 & & 191.8 & & & & & 254.4 & 254.6 & 243.6 & & & 234.3 & & & \\
\hline 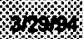 & $12: 00$ & 103.003 & 210.4 & & 223.8 & & 175.1 & & & & & 167.6 & 943.6 & 189.5 & & & 183.0 & & & \\
\hline 3. & $0: 00$ & 103.683 & 317.0 & & 246.8 & & 191.8 & & & & & 281.4 & 268.4 & 251.4 & & & 242.8 & & & \\
\hline
\end{tabular}


Table 5. Tensiometer Data

\begin{tabular}{|c|c|c|c|c|c|c|c|c|c|c|c|c|c|c|c|c|c|c|c|c|}
\hline & & & & & & & & & Ten & iometer & Data & & & & & & & & & \\
\hline & & Test & $\begin{array}{c}\text { Soll } \\
\text { Molsture } \\
\text { Tension } \\
\left(\mathrm{Cm} \mathrm{H}_{2} \mathrm{O}\right)\end{array}$ & $\begin{array}{c}\text { Soll } \\
\text { Molsture } \\
\text { Tension } \\
\left(\mathrm{Cm} \mathrm{H}_{2} \mathrm{O}\right)\end{array}$ & $\begin{array}{c}\text { Soli } \\
\text { Molsture } \\
\text { Tension } \\
\left(\mathrm{C} \mathrm{H} \mathrm{H}_{2} \mathrm{O}\right) \\
\end{array}$ & $\begin{array}{c}\text { Soil } \\
\text { Moisture } \\
\text { Tension } \\
\left(\mathrm{Cm} \mathrm{H}_{2} \mathrm{O}\right) \\
\end{array}$ & 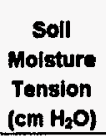 & $\begin{array}{c}\text { Soll } \\
\text { Moisture } \\
\text { Tension } \\
\left(\mathrm{cm} \mathrm{H}_{2} \mathrm{O}\right)\end{array}$ & $\begin{array}{c}\text { Soll } \\
\text { Molsture } \\
\text { Tension } \\
\left(\mathrm{Cm} \mathrm{H}_{2} \mathrm{O}\right) \\
\end{array}$ & 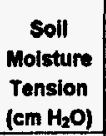 & $\begin{array}{c}\text { Soll } \\
\text { Molsture } \\
\text { Tension } \\
\left(\mathrm{cm} \mathrm{H} \mathrm{H}_{2} \mathrm{O}\right)\end{array}$ & $\begin{array}{c}\text { Soll } \\
\text { Moisture } \\
\text { Tension } \\
\left(\mathrm{Cm}_{2} \mathrm{O}\right) \\
\end{array}$ & $\begin{array}{c}\text { Soll } \\
\text { Molsture } \\
\text { Tension } \\
\left(\mathrm{em}^{2} \mathrm{H}_{2} \mathrm{O}\right) \\
\end{array}$ & $\begin{array}{c}\text { Soll } \\
\text { Molsture } \\
\text { Tension } \\
\left(\mathrm{em} \mathrm{H}_{2} \mathrm{O}\right) \\
\end{array}$ & $\begin{array}{c}\text { soll } \\
\text { Moisture } \\
\text { Tension } \\
\left(\mathrm{Em} \mathrm{H}_{2} \mathrm{O}\right)\end{array}$ & $\begin{array}{c}\text { Soll } \\
\text { Molsture } \\
\text { Tenslon } \\
\left(\mathrm{Cm} \mathrm{H}_{2} \mathrm{O}\right) \\
\end{array}$ & $\begin{array}{c}\text { Soll } \\
\text { Moisture } \\
\text { Tension } \\
\left(\mathrm{cm} \mathrm{H} \mathrm{H}_{2} \mathrm{O}\right) \\
\end{array}$ & $\begin{array}{c}\text { soll } \\
\text { Moisture } \\
\text { Tonsion } \\
\left.\text { (cm } \mathrm{H}_{2} \mathrm{O}\right) \\
\end{array}$ & $\begin{array}{c}\text { Soll } \\
\text { Molsture } \\
\text { Tension } \\
\left(\mathrm{cm} \mathrm{H} \mathrm{H}_{2} \mathrm{O}\right) \\
\end{array}$ & $\begin{array}{c}\text { Solf } \\
\text { Molsture } \\
\text { Tension } \\
\left(\mathrm{Cm} \mathrm{H}_{2} \mathrm{O}\right) \\
\end{array}$ \\
\hline Date & Time & Days' & $C 1^{\prime \prime}$ & $B 1^{\prime}$ & $A 1^{\prime \prime}$ & $C 2^{b}$ & $B 2^{b}$ & $A 2^{\mathbf{b}}$ & $\mathrm{Cs}^{\mathrm{C}}$ & $B 3^{c}$ & $\mathbf{A} 3^{\mathbf{c}}$ & $C 4^{d}$ & $B 4^{d}$ & $A 4^{d}$ & $C 5^{\circ}$ & $\mathrm{B5}^{\circ}$ & $A 5^{\circ}$ & $c 6^{\prime}$ & $\mathrm{B6}^{\prime}$ & $A 6^{\prime}$ \\
\hline$\%$ & $12: 00$ & 104.083 & 227.8 & & 230.8 & & 178.6 & & & & & 182.5 & 160.2 & 203.9 & & & 192.4 & & & \\
\hline $810 \%$ & 0.00 & 104.683 & 311.8 & & 246.0 & & 184.9 & & & & & 259.6 & 258.1 & 248.2 & & & 236.0 & & & \\
\hline$\gamma \psi$ & $12: 00$ & 105.083 & 197.3 & & 221.1 & & & & & & & 151.8 & 116.6 & 189.8 & & & 180.1 & & & \\
\hline 41,4 & $0: 00$ & 105.683 & 310.8 & & 247.6 & & & & & & & 254.2 & 269.2 & 253.0 & & & 233.2 & & & \\
\hline 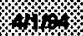 & $12: 00$ & 106.083 & 194.6 & & 223.6 & & & & & & & 147.4 & 113.0 & 194.9 & & & 182.6 & & & \\
\hline 14. & $0: 00$ & 106.683 & 313.4 & & 247.5 & & & & & & & 255.9 & 274.4 & 250.3 & & & 234.9 & & & \\
\hline 1.4. & $12: 00$ & 107.083 & 227.1 & & 223.8 & & & & & & & 178.0 & 139.3 & 194.1 & & & 192.4 & & & \\
\hline$\%$ & $0: 00$ & 107.683 & 320.7 & & 246.9 &. & & & & & & 263.8 & 279.1 & 247.8 & & & 243.1 & & & \\
\hline $4 \%$ & $12: 00$ & 108.083 & 196.7 & & 216.9 & & & & & & & 154.4 & 153.4 & 182.8 & & & 187.3 & & & \\
\hline $8 \%$ & $0: 00$ & 108.683 & 310.0 & & 247.6 & & & & & & & 252.4 & 257.9 & 244.1 & & & 229.8 & & & \\
\hline $14 \%$ & $12: 00$ & 109.083 & 195.8 & & 210.4 & & & & & & & 145.5 & 132.5 & 175.4 & & & 162.6 & & & \\
\hline $4 \%$ & $0: 00$ & 109.683 & 313.5 & & 249.4 & & & & & & & 254.1 & 281.5 & 246.8 & & & 234.2 & $\therefore$ & & \\
\hline 14.4 & $12: 00$ & 110.083 & 257.7 & & 238.9 & & & & & & & 206.0 & 199.6 & 205.6 & & & 199.5 & & & \\
\hline $1.4,4$. & $0: 00$ & 110.683 & 321.7 & & 247.9 & & & & & & & 264.8 & 270.5 & 243.5 & & & 245.0 & & & \\
\hline $14.9 \%$ & $12: 00$ & 111.083 & 216.6 & & 218.6 & & & & & & & 181.5 & 135.0 & 175.7 & & & 188.1 & & & \\
\hline 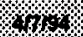 & $0: 00$ & 111.683 & 310.7 & & 251.9 & & & & & & & 253.1 & 255.2 & 246.7 & & & 230.5 & & & \\
\hline $17 \%$ & $12: 00$ & 112.083 & 241.7 & & 240.3 & & & & & & & 190.9 & 179.1 & 198.3 & & & 199.9 & & & \\
\hline $6 \%$ & $0: 00$ & 112.683 & 316.1 & & 252.0 & & & & & & & 258.4 & 284.9 & 245.8 & & & 238.5 & & & \\
\hline 1080 & $12: 00$ & 113.083 & 267.0 & & 245.6 & & & & & & & 213.7 & 205.3 & 209.7 & & & 209.5 & & & \\
\hline 4.414. & $0: 00$ & 113.683 & 304.8 & & 248.5 & & & & & & & 249.7 & 257.1 & 233.6 & & & 229.8 & & & \\
\hline $4 \%$ & $12: 00$ & 114.083 & 211.3 & & 217.6 & & & & & & & 164.7 & 156.6 & 178.2 & & & 194.0 & & & \\
\hline $160 \%$ & $0: 00$ & 114.683 & 314.4 & & 253.7 & & & & & & & 253.2 & 263.2 & 243.2 & & & 234.2 & & & \\
\hline 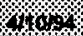 & $12: 00$ & 115.083 & 284.6 & & 247.5 & & & & & & & 229.5 & 231.7 & 217.7 & & & 217.6 & & & \\
\hline $4 \%$ & $0: 00$ & 115.683 & 313.7 & & 252.1 & & & & & & & 257.7 & 256.4 & 232.8 & & & 237.8 & & & \\
\hline (א. & $12: 00$ & 116.083 & 277.8 & & 242.4 & & & & & & & 227.8 & 217.0 & 209.0 & & & 214.2 & & & \\
\hline $1025 \%$ & $0: 00$ & 116.683 & 319.9 & & 258.3 & & & & & & & 262.1 & 258.9 & 239.9 & & & 239.6 & & & \\
\hline $4.1 \%$ & 12.00 & 117.083 & 228.2 & & 226.5 & & & & & & & 178.8 & 157.6 & 183.5 & & & 198.5 & & & \\
\hline 1) & $0: 00$ & 117.683 & 321.5 & & 261.8 & & & & & & & 262.1 & 267.7 & 246.9 & & & 238.7 & & & \\
\hline 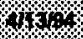 & $12: 00$ & 118.083 & 203.3 & & 217.5 & & & & & & & 160.4 & 139.1 & 173.8 & & & 190.4 & & & \\
\hline 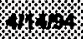 & $0: 00$ & 118.683 & 322.2 & & 284.2 & & & & & & & 258.5 & 270.1 & 252.9 & & & 234.8 & & & \\
\hline $2 / 4 \%$ & $12: 00$ & 119.083 & 212.8 & & 224.4 & & & & & & & 168.5 & 153.8 & 191.3 & & & 193.7 & & & \\
\hline $4 \%$ \% & $0: 00$ & 119.583 & 326.4 & & 268.5 & & & & & & & 261.9 & 278.0 & 262.5 & & & 240.0 & & & \\
\hline (4t) & $12: 00$ & 120.083 & 211.9 & & 220.0 & & & & & & & 169.8 & 152.0 & 194.7 & & & 204.0 & & & \\
\hline (x) & $0: 00$ & 120.683 & 336.7 & & 269.2 & & & & & & & 275.0 & 288.1 & 286.8 & & & 247.6 & & & \\
\hline 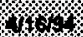 & $12: 00$ & 121.083 & 200.3 & & 218.1 & & & & & & & 169.0 & 150.1 & 191.2 & & * & 204.7 & & & \\
\hline 47 & $0: 00$ & 121.683 & 336.5 & & 270.8 & & & & & & & 274.8 & 286.9 & 264.1 & & 3 & 244.0 & & & \\
\hline 477 & $12: 00$ & 122.083 & 204.7 & & 218.0 & & & & & & & 163.7 & 145.6 & 200.8 & & & 201.1 & & & \\
\hline \%, & $0: 00$ & 122.583 & 334.6 & & 272.4 & & & & & & & 271.3 & 288.8 & 258.8 & & & 241.2 & & & \\
\hline
\end{tabular}


Table 5. Tensiometer Data

\begin{tabular}{|c|c|c|c|c|c|c|c|c|c|c|c|c|c|c|c|c|c|c|c|c|}
\hline & & & & & & & & & Ten & siometer & Data & & & & & & & & & \\
\hline & & Test & $\begin{array}{c}\text { Soll } \\
\text { Moisture } \\
\text { Tension } \\
\left(\mathrm{cm} \mathrm{H}_{2} \mathrm{O}\right) \\
\end{array}$ & $\begin{array}{c}\text { Solf } \\
\text { Moisture } \\
\text { Tenslon } \\
\left(\mathrm{cm} \mathrm{H}_{2} \mathrm{O}\right) \\
\end{array}$ & 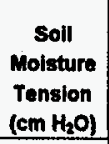 & $\begin{array}{c}\text { Soll } \\
\text { Molsture } \\
\text { Tension } \\
\left(\mathrm{C} \mathrm{H}_{2} \mathrm{O}\right)\end{array}$ & $\begin{array}{c}\text { Soll } \\
\text { Molsture } \\
\text { Tension } \\
\left(\mathrm{Cm} \mathrm{H}_{2} \mathrm{O}\right)\end{array}$ & $\begin{array}{c}\text { Soll } \\
\text { Molsture } \\
\text { Tension } \\
\left(\mathrm{Cm} \mathrm{H}_{2} \mathrm{O}\right) \\
\end{array}$ & $\begin{array}{c}\text { Soll } \\
\text { Molsture } \\
\text { Tension } \\
\left(\mathrm{Cm}_{2} \mathrm{O}\right) \\
\end{array}$ & $\begin{array}{c}\text { Soll } \\
\text { Moisture } \\
\text { Tension } \\
\left(\mathrm{Cm} \mathrm{H}_{2} \mathrm{O}\right) \\
\end{array}$ & $\begin{array}{c}\text { Soll } \\
\text { Molsture } \\
\text { Tension } \\
\left.\text { (cm } \mathrm{H}_{2} \mathrm{O}\right)\end{array}$ & $\begin{array}{c}\text { Soll } \\
\text { Molsture } \\
\text { Tenslon } \\
\left(\mathrm{Cm} \mathrm{H}_{2} \mathrm{O}\right)\end{array}$ & $\begin{array}{c}\text { Soil } \\
\text { Molsture } \\
\text { Tension } \\
\left(\mathrm{Cm} \mathrm{H}_{2} \mathrm{O}\right) \\
\end{array}$ & 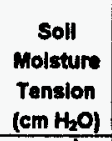 & $\begin{array}{c}\text { Soll } \\
\text { Molsture } \\
\text { Tension } \\
\left(\mathrm{cm} \mathrm{H}_{2} \mathrm{O}\right) \\
\end{array}$ & $\begin{array}{c}\text { Soll } \\
\text { Molsture } \\
\text { Tenslon } \\
\left(\mathrm{Cm} \mathrm{H}_{2} \mathrm{O}\right)\end{array}$ & $\begin{array}{c}\text { Soll } \\
\text { Molsture } \\
\text { Tension } \\
\left.\text { (em } \mathrm{H}_{2} \mathrm{O}\right) \\
\end{array}$ & $\begin{array}{c}\text { Soll } \\
\text { Moisture } \\
\text { Tenslon } \\
\left(\mathrm{Cm}_{2} \mathrm{H}_{2} \mathrm{O}\right)\end{array}$ & $\begin{array}{c}\text { Soll } \\
\text { Molsture } \\
\text { Tonslon } \\
\left(\mathrm{cm} \mathrm{H}_{2} \mathrm{O}\right) \\
\end{array}$ & $\begin{array}{c}\text { Soll } \\
\text { Moistur } \\
\text { Temsion } \\
\left(\mathrm{Cm} \mathrm{H}_{2} \mathrm{O}\right) \\
\end{array}$ \\
\hline Date & Time & Days $^{4}$ & $C 1^{a}$ & $B 1^{\prime \prime}$ & $A 1^{\prime \prime}$ & $\mathrm{Cl}^{\mathrm{b}}$ & $B 2^{6}$ & $A 2^{6}$ & $\mathrm{C3}^{\mathrm{c}}$ & $\mathrm{B3}^{\mathrm{C}}$ & $\mathrm{A3}^{\mathrm{C}}$ & $\mathrm{CA}^{\mathrm{d}}$ & $B 4^{\mathrm{d}}$ & $\mathrm{A4}^{\mathrm{d}}$ & $\mathrm{CE}^{\circ}$ & $\mathrm{B5}^{\circ}$ & $A 5^{\circ}$ & $\mathrm{Cb}^{\prime}$ & $B 6^{\prime}$ & $A 6^{\prime}$ \\
\hline \% & $12: 00$ & 123.083 & 210.7 & & 217.8 & & & & & & & 170.6 & 157.6 & 198.3 & & & 201.8 & & & \\
\hline$\%$ & $0: 00$ & 123.583 & 329.2 & & 271.4 & & & & & & & 267.7 & 282.2 & 252.5 & & & 240.1 & & & \\
\hline $1 \%$ & $12: 00$ & 124.083 & 218.4 & & 223.9 & & & & & & & 174.8 & 171.4 & 205.0 & & & 205.1 & & & \\
\hline$\%$ & $0: 00$ & 124.583 & 328.2 & & 268.8 & & & & & & & 268.5 & 278.7 & 251.6 & & & 241.8 & & & \\
\hline $4 \%$ & $12: 00$ & 126.083 & 203.6 & & 216.8 & & & & & & & 160.8 & 145.3 & 203.2 & & & 203.2 & & & \\
\hline$\%$ & $0: 00$ & 126.583 & 335.3 & & 271.4 & & & & & & & 271.2 & 288.6 & 253.4 & & $\cdots$ & 242.8 & & & \\
\hline \% & $12: 00$ & 128.083 & 190.5 & & 213.3 & & & & & & & 147.8 & 140.1 & 200.5 & & & 201.5 & & & \\
\hline 1. & 0.00 & 126.583 & 332.6 & & 274.8 & & & & & & & 271.1 & 286.4 & 249.8 & & & 240.0 & & & \\
\hline $12.2 \%$ & $12: 00$ & 127.083 & 197.5 & & 217.7 & & & & & & & 155.7 & 150.6 & 203.2 & & & 205.0 & & & \\
\hline $1223 \%$ & $0: 00$ & 127.583 & 336.1 & & 275.7 & & & & & & & 274.6 & 289.1 & 249.9 & & & 243.5 & & & \\
\hline 4.4 & $\begin{array}{l}12: 00 \\
\end{array}$ & 128.083 & 217.5 & & 225.6 & & & & & & . & 171.4 & 173.2 & 204.1 & & & 208.5 & & & \\
\hline 10,4 & 0.00 & 128.583 & 332.0 & & 271.5 & & & & & & & 273.0 & 283.3 & 244.7 & & & 242.0 & $=$ & & \\
\hline 144 & $12: 00$ & 129.083 & 213.3 & & 224.0 & & & & & & & 169.8 & 165.5 & 201.6 & & & 204.4 & & & \\
\hline$\%$ & 0.00 & 129.503 & 330.1 & & 275.8 & & & & & & & 268.6 & 279.7 & 241.2 & & & 238.3 & & & \\
\hline 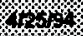 & $12: 00$ & 190.083 & 248.5 & & 235.7 & & & & & & & 198.8 & 194.6 & 203.6 & & & 207.5 & & & \\
\hline $8 \%$ & 0.00 & 130.583 & 330.1 & & 275.2 & & & & & & & 277.6 & 283.5 & 243.1 & & & 245.7 & & & \\
\hline $8128 \%$ & $12: 00$ & 131.083 & 231.8 & & 230.4 & & & & & & & 189.2 & 178.8 & 193.8 & & & 209.1 & & & \\
\hline$\%$ & 0.00 & 131.583 & 332.7 & & 281.8 & & & & & & & 275.6 & 278.7 & 238.6 & & & 241.8 & & & \\
\hline \%, & $12: 00$ & 192.083 & 320.7 & & 268.9 & & & & & & & 288.1 & 264.2 & 228.1 & & & 237.8 & & & \\
\hline 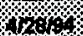 & 0.00 & 132.583 & 333.9 & & 274.3 & & & & & & & 279.3 & 273.0 & 234.4 & & & 247.4 & & & \\
\hline 1824 & $12: 00$ & .133 .083 & 278.8 & & 287.9 & & & & & & & 224.1 & 223.1 & 211.4 & & & 219.4 & & & \\
\hline 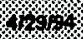 & $0: 00$ & 133.683 & 224.0 & & 280.2 & & 211.1 & & & 142.6 & 188.9 & 215.3 & 239.7 & 250.0 & & 169.0 & 235.0 & & & \\
\hline \%4 & $12: 00$ & 134.083 & 230.2 & & 271.5 & & 234.5 & & & 145.3 & 194.2 & 219.8 & 230.3 & 250.1 & & 187.4 & 236.9 & 189.2 & & \\
\hline 400 & $0: 00$ & 134.583 & 287.8 & & 281.5 & & 272.0 & & & 186.1 & 207.4 & 269.0 & 264.6 & 259.8 & & 228.0 & 256.4 & 235.2 & & \\
\hline 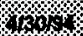 & $12: 00$ & 136.083 & & & 241.7 & & 203.2 & & & & 140.3 & 154.2 & 175.3 & 220.2 & & & 198.6 & 168.0 & & \\
\hline $4 \%$ & $0: 00$ & 136.583 & & & 287.2 & & 287.8 & & & 209.7 & 182.0 & 280.0 & 282.3 & 265.8 & & 248.4 & 285.3 & 248.7 & & \\
\hline 34 & $12: 00$ & 136.083 & & & 245.9 & & 223.8 & & & & & 172.5 & 192.5 & 224.5 & & 173.1 & 205.3 & 181.8 & & \\
\hline$\%$ & $0: 00$ & 136.583 & 280.6 & & 288.1 & & 296.6 & & & 218.6 & & 282.7 & 279.8 & 265.8 & & & 265.4 & 201.0 & & \\
\hline 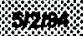 & $12: 00$ & 137.083 & 208.4 & & 258.2 & & 238.5 & & & & & 205.7 & 208.2 & 237.6 & & & 221.8 & 215.1 & & \\
\hline \% & $0: 00$ & 137.683 & 285.1 & & 290.8 & & 308.0 & & & 227.5 & & 289.7 & 285.1 & 269.4 & & & 269.8 & 268.0 & & \\
\hline (1) & $12: 00$ & 138.083 & & & 252.8 & & 234.8 & & & & & 185.5 & 199.4 & 231.4 & & & 213.0 & 200.8 & & \\
\hline $6 \%$ & $0: 00$ & 138.683 & 290.2 & & 293.4 & & 312.2 & & & 232.7 & & 294.0 & 289.4 & 272.8 & & & 275.0 & 275.1 & & \\
\hline 1,14 & \begin{tabular}{|l|}
$12: 00$ \\
\end{tabular} & 139.083 & & & 243.1 & & 213.2 & & & & & & 168.8 & 214.6 & & & 184.7 & 171.8 & & \\
\hline 4. & $0: 00$ & 139.683 & 289.0 & & 294.8 & & 310.8 & & & 230.7 & & 295.4 & 293.2 & 274.3 & & & 277.0 & 272.8 & & \\
\hline 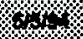 & $12: 00$ & 140.083 & & & 239.4 & & 214.8 & . & & & & & 174.7 & 214.5 & & & 194.5 & 170.8 & & \\
\hline 4 & $0: 00$ & 140.683 & 294.1 & & 296.4 & & 312.6 & & & 234.2 & & 286.3 & 295.8 & 277.8 & & 215.7 & 281.3 & 273.6 & & \\
\hline 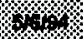 & $12: 00$ & 141.083 & & & 221.0 & & & 147.3 & 164.3 & & & & 160.7 & 196.8 & & & 184.9 & 175.0 & & \\
\hline xisu & $0: 00$ & 141.503 & 297.5 & 330.5 & 303.3 & 257.7 & 282.3 & 231.1 & 281.7 & & 188.8 & & 307.8 & 284.7 & 204.7 & & 288.1 & 280.5 & & \\
\hline
\end{tabular}


Table 5. Tensiometer Data

\begin{tabular}{|c|c|c|c|c|c|c|c|c|c|c|c|c|c|c|c|c|c|c|c|c|}
\hline & & & & & & & & & Ten: & iometer & Data & & & & & & & & & \\
\hline & & Test & $\begin{array}{c}\text { Soll } \\
\text { Moisture } \\
\text { Tension } \\
\left(\mathrm{Cm} \mathrm{H}_{2} \mathrm{O}\right) \\
\end{array}$ & $\begin{array}{c}\text { Soil } \\
\text { Moisture } \\
\text { Tension } \\
\left(\mathrm{Cm} \mathrm{H}_{2} \mathrm{O}\right) \\
\end{array}$ & $\begin{array}{c}\text { Soil } \\
\text { Molsture } \\
\text { Tension } \\
\left(\mathrm{Cm} \mathrm{H}_{2} \mathrm{O}\right) \\
\end{array}$ & $\begin{array}{c}\text { Soll } \\
\text { Molsture } \\
\text { Tension } \\
\left(\mathrm{Cm} \mathrm{H}_{2} \mathrm{O}\right) \\
\end{array}$ & $\begin{array}{c}\text { Soll } \\
\text { Moisture } \\
\text { Tension } \\
\left(\mathrm{Cm} \mathrm{H}_{2} \mathrm{O}\right) \\
\end{array}$ & $\begin{array}{c}\text { Soll } \\
\text { Moisture } \\
\text { Tension } \\
\left(\mathrm{Cm} \mathrm{H}_{2} \mathrm{O}\right) \\
\end{array}$ & $\begin{array}{c}\text { Soll } \\
\text { Molsture } \\
\text { Tenslon } \\
\left(\mathrm{cm} \mathrm{H}_{2} \mathrm{O}\right) \\
\end{array}$ & $\begin{array}{c}\text { soll } \\
\text { Moisture } \\
\text { Tenslon } \\
\left(\mathrm{Cm} \mathrm{H}_{2} \mathrm{O}\right) \\
\end{array}$ & 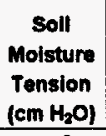 & $\begin{array}{c}\text { Soll } \\
\text { Moisture } \\
\text { Tension } \\
\left(\mathrm{em} \mathrm{H}_{2} \mathrm{O}\right) \\
\end{array}$ & $\begin{array}{c}\text { Soll } \\
\text { Molsture } \\
\text { Tonsion } \\
\left(\mathrm{Cm} \mathrm{H}_{2} \mathrm{O}\right) \\
\end{array}$ & $\begin{array}{c}\text { Soll } \\
\text { Moisture } \\
\text { Tension } \\
\left(\mathrm{Cm} \mathrm{H}_{2} \mathrm{O}\right) \\
\end{array}$ & $\begin{array}{c}\text { Soll } \\
\text { Moisture } \\
\text { Tension } \\
\left(\mathrm{cm} \mathrm{H}_{2} \mathrm{O}\right) \\
\end{array}$ & $\begin{array}{c}\text { Soll } \\
\text { Molsture } \\
\text { Tension } \\
\left(\mathrm{Cm} \mathrm{H}_{2} \mathrm{O}\right) \\
\end{array}$ & $\begin{array}{c}\text { Soll } \\
\text { Molsture } \\
\text { Tension } \\
\left(\mathrm{cm} \mathrm{H}_{2} \mathrm{O}\right) \\
\end{array}$ & $\begin{array}{c}\text { Soll } \\
\text { Molsture } \\
\text { Tension } \\
\left.\text { (cm } \mathrm{H}_{2} \mathrm{O}\right) \\
\end{array}$ & $\begin{array}{c}\text { Soll } \\
\text { Molsture } \\
\text { Tension } \\
\left(\mathrm{Cm} \mathrm{H}_{2} \mathrm{O}\right)\end{array}$ & $\begin{array}{c}\text { Soll } \\
\text { Molsture } \\
\text { Tension } \\
\left(\mathrm{cm} \mathrm{H}_{2} \mathrm{O}\right) \\
\end{array}$ \\
\hline Date & Time & Days' & $\mathrm{C1}^{\prime \prime}$ & $B 1^{2}$ & $\mathrm{A1}^{*}$ & $\mathrm{C2}^{\mathrm{b}}$ & $\mathrm{B2}^{\mathrm{b}}$ & $A 2^{b}$ & $c 3^{c}$ & $B 3^{\mathrm{C}}$ & $A 3^{c}$ & $\mathrm{CA}^{\mathrm{d}}$ & $B 4^{d}$ & $A 4^{d}$ & $\mathrm{Cb}^{\circ}$ & $\mathrm{B5} 5^{\circ}$ & $A 5^{\circ}$ & $c 6^{\prime}$ & $B 6^{\prime}$ & $A 6^{\prime}$ \\
\hline KM. & $12: 00$ & 142.083 & 254.9 & 277.5 & 273.6 & 248.9 & 259.8 & 232.9 & 256.4 & & 193.0 & & 262.6 & 252.3 & 208.0 & & 249.1 & 256.7 & & \\
\hline (3) & $0: 00$ & 142.683 & 282.9 & 294.3 & 290.4 & 278.1 & 285.1 & 252.2 & 279.3 & & 199.2 & & 280.3 & 269.1 & 230.0 & & 285.9 & 276.4 & & \\
\hline 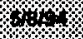 & 12:00 & 143.083 & & & 228.8 & 190.5 & 171.6 & 188.3 & 204.2 & & 152.2 & & 156.5 & 207.5 & 192.2 & & 188.4 & 176.0 & & \\
\hline 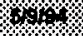 & $0: 00$ & 143.583 & 281.9 & 319.3 & 300.7 & 286.4 & 293.5 & 262.5 & 288.5 & & 208.6 & & 293.1 & 278.6 & 243.6 & & 277.7 & 272.6 & & \\
\hline$\%$ & $12: 00$ & 144.083 & 200.1 & 229.8 & 258.5 & 239.2 & 236.4 & 221.5 & 239.0 & & 180.0 & & 217.3 & 234.7 & 217.4 & & 222.2 & 219.8 & & \\
\hline 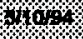 & 0.00 & 144.583 & 289.0 & 302.1 & 302.6 & 297.0 & 304.9 & 273.1 & 294.8 & & 213.1 & & 292.4 & 278.6 & 253.3 & & 279.7 & 279.0 & & \\
\hline 80.4 & $12: 00$ & 146.083 & 251.6 & 261.3 & 274.7 & 278.8 & 275.6 & 260.0 & 275.8 & & 200.9 & & 253.4 & 250.7 & 239.5 & & 246.9 & 258.0 & & \\
\hline $4 \%$ & $0: 00$ & 146.583 & 277.0 & 281.4 & 293.2 & 295.4 & 303.4 & 273.2 & 287.2 & & 212.3 & & 273.5 & 265.7 & 252.0 & & 281.7 & 275.7 & & \\
\hline 614104 & $12: 00$ & 146.083 & 213.3 & 228.1 & 266.7 & 240.3 & 258.0 & 234.7 & 245.3 & & 181.0 & & 224.5 & 243.6 & 223.7 & & 232.0 & 229.8 & & \\
\hline 84394 & 0.00 & 146.583 & 276.2 & 291.1 & 298.6 & 298.2 & 307.0 & 273.3 & 287.4 & & 213.3 & & 271.1 & 266.7 & 253.7 & & 283.6 & 274.1 & & \\
\hline 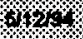 & $12: 00$ & 197.083 & 249.3 & 267.7 & 283.8 & 280.9 & 292.4 & 263.8 & 272.7 & & 202.9 & & 252.0 & 254.5 & 243.4 & & 250.7 & 255.0 & & \\
\hline (1) & 0.00 & 147.583 & 264.1 & 289.5 & 296.9 & 291.3 & 300.2 & 266.4 & 280.5 & & 211.6 & & 264.2 & 267.7 & 248.6 & & 259.4 & 265.5 & & \\
\hline (5) & $12: 00$ & 148.083 & & 197.1 & 243.3 & 200.5 & 211.7 & 194.7 & 196.8 & & 149.0 & & 169.1 & 217.5 & 202.3 & & & & & \\
\hline 41048 & $0: 00$ & 148.583 & & 307.7 & 311.8 & 299.1 & 307.0 & 272.5 & 293.5 & & 212.5 & & 286.8 & 279.8 & 255.5 & & 275.0 & 240.9 & & \\
\hline $34 \%$ & $12: 00$ & 149.083 & & 189.2 & 243.2 & 202.1 & 208.4 & 192.0 & 200.3 & & 143.7 & & 172.4 & 218.3 & 207.4 & & & & & \\
\hline 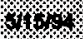 & $0: 00$ & 149.583 & & 320.6 & 317.8 & 308.6 & 316.4 & 282.9 & 307.3 & & 203.7 & & 303.2 & 288.7 & 262.3 & & & 220.6 & & \\
\hline $818 \%$ & $12: 00$ & 150.083 & & 194.4 & 245.8 & 211.6 & 216.8 & 199.8 & 207.1 & & 133.3 & & 180.2 & 221.8 & 200.9 & & 212.1 & & & \\
\hline $4 \%$ & 0.00 & 150.583 & & 308.4 & 310.7 & 304.2 & 314.6 & 280.3 & 303.8 & & 203.7 & & 293.6 & 280.7 & 259.6 & & 277.3 & 214.4 & & \\
\hline 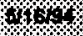 & $12: 00$ & 151.083 & & 200.4 & 253.6 & 213.3 & 225.3 & 203.3 & 209.7 & & 134.1 & & 188.8 & 228.8 & 208.9 & & 218.9 & & & \\
\hline $647 \%$ & 0.00 & 161.583 & & 310.0 & 315.0 & 305.8 & 320.6 & 281.8 & 307.1 & & 204.5 & & 296.9 & 285.0 & 262.1 & & 281.5 & 223.0 & & \\
\hline 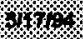 & $12: 00$ & 162.083 & & 185.5 & 246.4 & 205.3 & 214.8 & 198.2 & 206.0 & & 131.4 & & 173.8 & 221.6 & 206.2 & & 211.8 & & & \\
\hline 414 & $0: 00$ & 162.583 & & 317.5 & 317.2 & 308.9 & 321.0 & 288.8 & 311.0 & & 209.5 & & 303.4 & 290.0 & 268.0 & & 285,4 & 235.8 & & \\
\hline 46 & $13: 52$ & 161.161 & & 277.3 & 296.1 & 313.2 & 321.0 & 283.0 & 300.6 & & 205.8 & & 276.5 & 272.1 & 263.4 & & 265.6 & & & \\
\hline 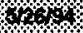 & $13: 53$ & 161.162 & & 277.4 & 296.1 & 313.2 & 321.8 & 283.9 & 300.8 & & 204.7 & & 276.5 & 272.1 & 263.4 & & 265.0 & & & \\
\hline 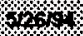 & 13.54 & 161.163 & & 275.6 & 296.1 & 313.2 & 321.0 & 283.0 & 300.6 & & 204.7 & & 275.6 & 272.1 & 263.4 & & 266.4 & & & \\
\hline $47214 \%$ & 0.00 & 161.583 & & 293.1 & 308.7 & 322.8 & 334.0 & 283.9 & 311.1 & & 212.6 & & 284.4 & 279.2 & 273.9 & & 271.7 & & & \\
\hline$\%$ & $12: 00$ & 162.083 & & 184.2 & 253.0 & 224.1 & 245.5 & 219.2 & 221.3 & & 152.5 & & 191.8 & 235.1 & 218.0 & & 224.5 & & & \\
\hline $42 \%$ & $0: 00$ & 102.583 & & 324.3 & 320.6 & 324.5 & 331.4 & 296.2 & 318.0 & & 212.8 & & 300.8 & 295.9 & 278.4 & & 285.5 & & & \\
\hline 3244 & $12: 00$ & 163.083 & & 183.2 & 250.3 & 213.6 & 233.3 & 212.2 & 210.8 & & 145.6 & & 190.0 & 232.5 & 214.5 & & 225.3 & & & \\
\hline 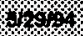 & $0: 00$ & 163.583 & & 328.4 & 324.7 & 319.0 & 325.9 & 292.6 & 315.1 & & 206.4 & & 304.9 & 301.0 & 271.8 & & 288.6 & & & \\
\hline $4=4$ & $12: 00$ & 164.083 & & 191.9 & 248.5 & 227.4 & 228.9 & 205.2 & 215.1 & & 140.3 & & 190.8 & 231.6 & 215.2 & & 227.8 & & & \\
\hline $8 \%$ & $0: 00$ & 164.583 & & 343.3 & 329.2 & 328.7 & 334.7 & 302.2 & 325.7 & & 209.8 & & 317.2 & 308.9 & 277.1 & & 298.6 & & & \\
\hline $1180 \%$ & $12: 00$ & 105.083 & & 172.7 & 240.6 & 200.4 & 222.0 & 197.3 & 201.1 & & 136.8 & & 183.8 & 224.5 & 203.9 & & 222.6 & & & \\
\hline 5018 & $0: 00$ & 165.683 & & 330.9 & 323.8 & 325.9 & 332.7 & 300.2 & 322.8 & & 210.6 & & 314.3 & 308.0 & 273.4 & & 292.8 & & & \\
\hline 613 & $12: 00$ & 166.083 & & 246.6 & 273.0 & 272.7 & 283.4 & 253.1 & 268.0 & & 175.8 & & 251.6 & 257.8 & 242.1 & & 252.9 & & & \\
\hline 4 & 0.00 & 166.583 & & 322.3 & 317.7 & 328.5 & 335.3 & 302.0 & 323.7 & & 212.3 & & 309.2 & 303.4 & 278.0 & & 292.0 & & & \\
\hline 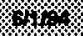 & $12: 00$ & 167.083 & & 183.9 & 247.5 & 225.5 & 233.1 & 218.1 & 211.5 & & & & 104.1 & 235.7 & 212.5 & & 231.1 & & & \\
\hline 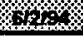 & $0: 00$ & 107.589 & & 324.8 & 316.8 & 329.3 & 338.8 & 301.8 & 325.4 & & 210.5 & & 311.7 & 300.7 & 276.9 & & 291.1 & & & \\
\hline
\end{tabular}


Table 5. Tensiometer Data

\begin{tabular}{|c|c|c|c|c|c|c|c|c|c|c|c|c|c|c|c|c|c|c|c|c|}
\hline & & & & & & & & & Ten & iometer & Data & & & & & & & & & \\
\hline & & Test & $\begin{array}{c}\text { Soll } \\
\text { Molsture } \\
\text { Tension } \\
\text { (cm } \mathrm{H}_{2} \mathrm{O} \text { ) } \\
\end{array}$ & $\begin{array}{l}\text { Soll } \\
\text { Moisture } \\
\text { Tension } \\
\left(\mathrm{cm} \mathrm{H}_{2} \mathrm{O}\right) \\
\end{array}$ & $\begin{array}{c}\text { Soll } \\
\text { Molsture } \\
\text { Tension } \\
\left(\mathrm{Cm} \mathrm{H}_{2} \mathrm{O}\right)\end{array}$ & $\begin{array}{c}\text { Soll } \\
\text { Molsture } \\
\text { Tension } \\
\left(\mathrm{Cm}_{2} \mathrm{O}\right) \\
\end{array}$ & $\begin{array}{c}\text { Soll } \\
\text { Molsture } \\
\text { Tension } \\
\left(\mathrm{Cm} \mathrm{H}_{2} \mathrm{O}\right)\end{array}$ & $\begin{array}{c}\text { Soll } \\
\text { Molsture } \\
\text { Tension } \\
\left(\mathrm{cm} \mathrm{H}_{2} \mathrm{O}\right)\end{array}$ & 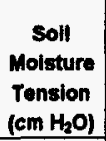 & $\begin{array}{c}\text { Soll } \\
\text { Molsture } \\
\text { Tension } \\
\left(\mathrm{Cm} \mathrm{H}_{2} \mathrm{O}\right) \\
\end{array}$ & $\begin{array}{c}\text { Soll } \\
\text { Molsture } \\
\text { Tension } \\
\left(\mathrm{Cm} \mathrm{H}_{2} \mathrm{O}\right) \\
\end{array}$ & 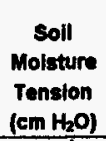 & $\begin{array}{c}\text { Soll } \\
\text { Molsture } \\
\text { Tenslon } \\
\left(\mathrm{cm} \mathrm{H}_{2} \mathrm{O}\right) \\
\end{array}$ & $\begin{array}{c}\text { Soll } \\
\text { Molsture } \\
\text { Tension } \\
\left.\text { (em } \mathrm{H}_{2} \mathrm{O}\right) \\
\end{array}$ & $\begin{array}{c}\text { Soll } \\
\text { Molature } \\
\text { Tension } \\
\left.\text { (cm } \mathrm{H}_{2} \mathrm{O}\right) \\
\end{array}$ & $\begin{array}{c}\text { Soll } \\
\text { Molnture } \\
\text { Tonslon } \\
\left(\mathrm{cm} \mathrm{H}_{2} \mathrm{O}\right) \\
\end{array}$ & $\begin{array}{c}\text { Soll } \\
\text { Molsture } \\
\text { Tension } \\
\left(\mathrm{Cm} \mathrm{H}_{2} \mathrm{O}\right) \\
\end{array}$ & $\begin{array}{c}\text { Soll } \\
\text { Moisture } \\
\text { Tanslon } \\
\left(\mathrm{cm} \mathrm{H}_{2} \mathrm{O}\right) \\
\end{array}$ & $\begin{array}{c}\text { Soll } \\
\text { moisture } \\
\text { Tension } \\
\left.\text { (em } \mathrm{H}_{2} \mathrm{O}\right) \\
\end{array}$ & $\begin{array}{c}\text { soll } \\
\text { moisture } \\
\text { Tension } \\
\left(\mathrm{cm} \mathrm{H} \mathrm{H}_{2} \mathrm{O}\right) \\
\end{array}$ \\
\hline Date & Time & Days' & $C 1^{a}$ & $B 1^{\prime \prime}$ & $A 1^{\prime \prime}$ & $\mathrm{C2}^{\mathrm{b}}$ & $\mathrm{B2}^{6}$ & $A 2^{b}$ & $C 3^{c}$ & $\mathrm{B3}^{\mathrm{C}}$ & $A 3^{c}$ & $\mathrm{CA}^{\mathrm{d}}$ & $\mathrm{BA}^{\mathrm{d}}$ & $A 4^{d}$ & $C 5^{\circ}$ & $B 6^{\circ}$ & $A 5^{\circ}$ & $c 6^{\prime}$ & $B 6^{\prime}$ & $A 6^{\prime}$ \\
\hline $0 \%$ & $0: 56$ & 173.622 & & 148.0 & 228.8 & 198.1 & 200.7 & 178.6 & 194.4 & & & & 164.0 & 218.7 & 202.2 & & 214.8 & & & \\
\hline $8 \% 6 \%$ & $0: 57$ & 173.623 & & 148.0 & 228.6 & 198.1 & 205.8 & 178.6 & 191.8 & & & & 184.0 & 217.8 & 202.2 & & 214.8 & & & \\
\hline $1 \%$ & $0: 58$ & 173.624 & & 148.0 & 227.8 & 198.3 & 205.0 & 178.6 & 190.1 & & & & 164.0 & 217.9 & 202.2 & & 214.8 & & & \\
\hline $1 \%$ & \begin{tabular}{|l|}
$0: 59$ \\
\end{tabular} & 173.624 & & 147.1 & 227.8 & 195.4 & 205.0 & 178.6 & 189.2 & & & & 163.1 & 218.7 & 201.4 & & 214.8 & & & \\
\hline 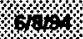 & 22:00 & 174.083 & & 333.8 & 323.2 & 350.5 & 358.0 & 326.1 & 343.8 & & & & 335.4 & 320.8 & 289.2 & & 308.9 & & & \\
\hline 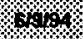 & $0: 00$ & 174.683 & & 148.0 & 221.6 & 200.7 & 216.1 & 189.1 & 201.3 & & & & 171.7 & 217.8 & 206.5 & & 217.3 & & & \\
\hline 8649 & 12:00 & 175.083 & & 335.2 & 321.2 & 345.8 & 351.6 & 322.4 & 339.1 & & & & 333.2 & 321.2 & 284.5 & & 305.6 & & & \\
\hline $1810 \%$ & $0: 00$ & 175.683 & & 142.7 & 218.0 & 202.2 & 204.8 & 178.5 & 198.5 & & & & 163.8 & 213.4 & 208.0 & & 213.7 & & & \\
\hline 140\% & $12: 00$ & 176.083 & & 342.0 & 322.8 & 345.6 & 350.6 & 325.0 & 340.7 & & & & 335.7 & 323.8 & 283.4 & & 307.1 & & & \\
\hline $84 \times 14$ & $0: 00$ & 176.683 & & 150.5 & 223.2 & 203.9 & 213.4 & 187.2 & 196.8 & & & & 175.9 & 217.8 & 208.3 & & 297.8 & & & \\
\hline $8 \% 11 \%$ & $12: 00$ & 177.083 & & 336.6 & 316.5 & 341.8 & 345.2 & 318.8 & 337.0 & & & & 330.2 & 320.2 & 279.8 & & 304.3 & & & \\
\hline 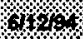 & $0: 00$ & 177.683 & & 150.4 & 217.1 & 192.8 & 209.9 & 182.0 & 189.0 & & & & 172.4 & 216.0 & 215.7 & & 216.2 & 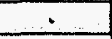 & & \\
\hline (1) & $12: 00$ & 178.083 & & 345.5 & 321.1 & 352.8 & 354.0 & 328.5 & 345.9 & & & & 339.2 & 324.7 & 285.2 & & 308.9 & & & \\
\hline 8414 & $0: 00$ & 178.683 & & 147.8 & 215.3 & 209.9 & 208.1 & 182.0 & 204.5 & & & & 167.1 & 213.3 & 204.4 & & 212.7 & & & \\
\hline $4 \%$ & $12: 00$ & 179.083 & & 344.5 & 323.6 & 352.4 & 353.1 & 320.6 & 345.7 & & & & 339.0 & 328.1 & 285.0 & & 300.8 & & & \\
\hline $1144 \%$ & $0: 00$ & 179.683 & & 172.1 & 232.7 & 231.6 & 235.7 & 213.4 & 229.6 & & & & 183.1 & 231.7 & 214.8 & & 225.6 & & & \\
\hline $844 \%$ & 12:00 & 180.083 & & 332.4 & 323.6 & 355.1 & 359.1 & 329.3 & 349.2 & & & & 340.8 & 325.5 & 290.2 & & 311.3 & & & \\
\hline $8 \%$ & 000 & 180.683 & & 144.4 & 217.1 & 216.8 & 216.0 & 192.5 & 201.1 & & & & 172.4 & 216.0 & 207.1 & & 213.6 & & & \\
\hline \%) & $12: 00$ & 181.083 & & 343.8 & 328.1 & 358.7 & 362.7 & 328.5 & 352.8 & & & & 344.4 & 328.1 & 293.8 & & 313.2 & & & \\
\hline 14 & $0: 00$ & 181.693 & & 141.8 & 222.4 & 225.6 & 222.9 & 193.3 & 216.7 & & & & 177.6 & 218.6 & 214.9 & & 211.9 & & & \\
\hline 86164 & $12: 00$ & 182.083 & & 343.7 & 328.9 & 358.8 & 362.8 & 335.4 & 347.6 & & & & 342.8 & 327.3 & 291.2 & & 309.7 & & & \\
\hline 876 & $0: 00$ & 182.683 & & 169.4 & 240.5 & 253.1 & 245.0 & 216.7 & 240.8 & & & & 206.0 & 231.6 & 230.2 & & 222.9 & & & \\
\hline 817.8 & $12: 00$ & 783.083 & & 340.1 & 326.1 & 351.5 & 353.8 & 326.6 & 342.2 & & & & 335.4 & 323.6 & 286.7 & & 305.2 & & & \\
\hline $1418 \%$ & $0: 00$ & 183.683 & & 153.8 & 224.0 & 217.7 & 234.8 & 198.5 & 207.9 & & & & 193.1 & 215.0 & 211.3 & & 216.8 & & & \\
\hline $8710 \%$ & $12: 00$ & 184.083 & & 318.6 & 314.8 & 348.5 & 353.2 & 324.8 & 337.2 & & & & 324.4 & 308.0 & 288.0 & & 295.9 & & & \\
\hline 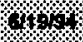 & $0: 00$ & 184.683 & & 149.6 & 227.5 & 213.4 & 215.0 & 187.2 & 199.3 & & & & 167.2 & 215.8 & 225.2 & & 207.5 & & & \\
\hline $81 \%$ & 12:00 & 185.083 & & 343.9 & 328.4 & 350.2 & 354.2 & 332.0 & 340.0 & & & & 330.7 & 318.6 & 288.0 & & 305.6 & & & \\
\hline $6 \%$ & $0: 00$ & 185.683 & & 209.4 & 257.3 & 268.2 & 260.0 & 241.3 & 244.5 & & & & 221.1 & 244.9 & 229.8 & & 248.2 & & & \\
\hline 862.6 & $12: 00$ & 186.083 & & 329.2 & 323.8 & 344.8 & 349.0 & 322.4 & 336.5 & & & & 324.6 & 311.6 & 284.5 & & 296.1 & & & \\
\hline $86 \%$ & $0: 00$ & 986.683 & & 193.1 & 261.9 & 252.9 & 269.7 & 227.5 & 236.8 & & & & 231.8 & 248.6 & 226.6 & & 248.5 & & & \\
\hline $14+1 \%$ & $12: 00$ & 187.003 & & 335.7 & 328.9 & 348.0 & 353.0 & 325.4 & 340.5 & & & & 325.1 & 312.0 & 289.3 & & 298.4 & & & \\
\hline 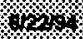 & $0: 00$ & 187.683 & & 142.8 & 239.4 & 211.4 & 207.8 & 182.8 & 200.1 & & & & 161.8 & 218.5 & 198.6 & & 215.3 & & & \\
\hline $100 \%$ & $12: 00$ & 988.083 & & 333.3 & 332.3 & 346.6 & 350.7 & 325.4 & 339.0 & & & & 325.4 & 317.3 & 287.0 & & 299.5 & & & \\
\hline 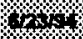 & $0: 00$ & 188.683 & & 163.0 & 247.3 & 230.5 & 224.3 & 204.0 & 219.0 & & & & 176.5 & 230.3 & 217.4 & & 230.8 & & & \\
\hline$\%$ & \begin{tabular}{|l|}
$12: 00$ \\
\end{tabular} & 189.083 & & 342.7 & 340.9 & 351.6 & 352.2 & 331.5 & 342.3 & & & & 332.2 & 328.9 & 286.8 & & 303.7 & & & \\
\hline $8 \mathrm{z}$ & $0: 00$ & 180.683 & & 148.1 & 241.0 & 207.8 & 207.8 & 192.7 & 192.0 & & & & 187.6 & 224.2 & 208.0 & 3 & 226.3 & & & \\
\hline 8) & $12: 00$ & 100.083 & & 351.9 & 343.2 & 347.8 & 347.5 & 330.4 & 341.1 & & & & 334.4 & 330.1 & 283.0 & & 305.0 & & & \\
\hline 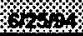 & $0: 00$ & 180.603 & & 145.4 & 233.0 & 190.2 & 192.9 & 179.5 & 175.4 & & & & 154.4 & 215.2 & 213.5 & & 221.7 & & & \\
\hline
\end{tabular}


Table 5. Tensiometer Data

\begin{tabular}{|c|c|c|c|c|c|c|c|c|c|c|c|c|c|c|c|c|c|c|c|c|}
\hline & & & & & & & & & Ten & siometer & Data & & & & & & & & & \\
\hline & & Test & $\begin{array}{c}\text { Soll } \\
\text { Moisture } \\
\text { Tension } \\
\left(\mathrm{Cm} \mathrm{H}_{2} \mathrm{O}\right) \\
\end{array}$ & $\begin{array}{c}\text { Soll } \\
\text { Moisture } \\
\text { Tension } \\
\left(\mathrm{Cm} \mathrm{H}_{2} \mathrm{O}\right) \\
\end{array}$ & 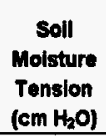 & $\begin{array}{c}\text { Soll } \\
\text { Molsture } \\
\text { Tension } \\
\left(\mathrm{cm} \mathrm{H}_{2} \mathrm{O}\right) \\
\end{array}$ & $\begin{array}{c}\text { Soll } \\
\text { Moisture } \\
\text { Tension } \\
\left(\mathrm{em} \mathrm{H}_{2} \mathrm{O}\right) \\
\end{array}$ & $\begin{array}{c}\text { Soll } \\
\text { Moisture } \\
\text { Tension } \\
\left(\mathrm{cm} \mathrm{H}_{2} \mathrm{O}\right)\end{array}$ & $\begin{array}{c}\text { Soil } \\
\text { Moisture } \\
\text { Tension } \\
\left(\mathrm{Cm}_{2} \mathrm{O}\right) \\
\end{array}$ & 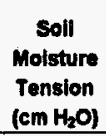 & $\begin{array}{c}\text { Soll } \\
\text { Moisture } \\
\text { Tension } \\
\left(\mathrm{cm} \mathrm{H}_{2} \mathrm{O}\right) \\
\end{array}$ & $\begin{array}{c}\text { Soil } \\
\text { Molsture } \\
\text { Tension } \\
\left(\mathrm{Cm} \mathrm{H}_{2} \mathrm{O}\right) \\
\end{array}$ & $\begin{array}{c}\text { Soll } \\
\text { Molisture } \\
\text { Tenslon } \\
\left(\mathrm{cm} \mathrm{H}_{2} \mathrm{O}\right) \\
\end{array}$ & $\begin{array}{c}\text { Soll } \\
\text { Molsture } \\
\text { Tension } \\
\left(\mathrm{Cm} \mathrm{H}_{2} \mathrm{O}\right) \\
\end{array}$ & \begin{tabular}{|c|} 
Soll \\
Molsture \\
Tension \\
$\left(\mathrm{Cm} \mathrm{H}_{2} \mathrm{O}\right)$ \\
\end{tabular} & $\begin{array}{c}\text { Soll } \\
\text { Molsture } \\
\text { Tonaion } \\
\left(\mathrm{Cm} \mathrm{H}_{2} \mathrm{O}\right) \\
\end{array}$ & 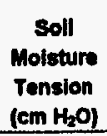 & $\begin{array}{c}\text { Soll } \\
\text { Molsture } \\
\text { Tension } \\
\left(\mathrm{cm} \mathrm{H}_{2} \mathrm{O}\right) \\
\end{array}$ & $\begin{array}{c}\text { Soll } \\
\text { Molsture } \\
\text { Tenslon } \\
(\mathrm{cm} \mathrm{H} \mathrm{O})\end{array}$ & $\begin{array}{c}\text { Solf } \\
\text { Molsture } \\
\text { Tension } \\
\left(\mathrm{Cm} \mathrm{H}_{3} \mathrm{O}\right) \\
\end{array}$ \\
\hline Date & Time & Days ${ }^{1}$ & $C 1^{*}$ & $\mathrm{B1}^{\prime \prime}$ & $A 1^{2}$ & $\mathrm{C2}^{\mathrm{b}}$ & $B 2^{6}$ & $A 2^{b}$ & $\mathrm{C3}^{\mathrm{C}}$ & $\mathrm{B3}^{\mathrm{C}}$ & $A 3^{\mathrm{C}}$ & $\mathrm{CA}^{\mathrm{d}}$ & $B 4^{d}$ & $\mathrm{AM}^{\mathrm{d}}$ & $\mathrm{C5}^{\circ}$ & $85^{\circ}$ & $A 5^{\circ}$ & $\mathrm{Co}^{\prime}$ & $86^{\prime}$ & $A 6^{\prime}$ \\
\hline ons & $12: 00$ & 191.083 & & 368.4 & 351.0 & 357.3 & 355.3 & 339.8 & 353.2 & & & & 343.9 & 338.0 & 287.3 & & 311.8 & & & \\
\hline 1018.4 & 0.00 & 191.683 & & 154.8 & 233.8 & 204.1 & 201.5 & 187.2 & 193.5 & & & & 166.5 & 218.6 & 206.5 & & 225.8 & & & \\
\hline $6 \%$ & $12: 00$ & 192.083 & & 367.2 & 347.2 & 357.0 & 354.1 & 337.9 & 351.1 & & & & 344.4 & 336.8 & 285.2 & & 308.1 & & & \\
\hline 1874 & 0.00 & 102.583 & & 171.3 & 239.0 & 226.6 & 214.3 & 199.4 & 217.8 & & & & 177.7 & 227.3 & 225.4 & & 231.9 & & & \\
\hline 68764 & $12: 00$ & 103.083 & & 359.3 & 340.2 & 356.0 & 355.7 & 338.7 & 351.0 & & & & 344.3 & 330.6 & 286.9 & & 308.0 & & & \\
\hline $62 \% 4$ & \begin{tabular}{|l|l|} 
\\
\end{tabular} & 193.683 & & 171.4 & 238.2 & 223.2 & 228.5 & 207.2 & 217.7 & & & & 194.3 & 229.0 & 227.2 & & 235.4 & & & \\
\hline 6 & $12: 00$ & 194.083 & & 351.2 & 337.2 & 360.9 & 360.8 & 337.9 & 355.1 & & & & 343.2 & 329.8 & 290.1 & & 308.6 & & & \\
\hline $429 \%$ & $0: 00$ & 194.683 & & 148.1 & 225.3 & 206.8 & 208.0 & 187.1 & 197.1 & & & & 168.7 & 216.7 & 222.3 & & 222.7 & & & \\
\hline $202 \%$ & $12: 00$ & 195.083 & & 358.0 & 334.5 & 359.1 & 357.9 & 339.7 & 353.3 & & & & 341.4 & 329.8 & 290.8 & & 308.5 & & & \\
\hline 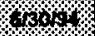 & $0: 00$ & 195.683 & & 143.7 & 221.7 & 203.2 & 203.2 & 186.3 & 193.5 & & & & 157.9 & 215.8 & 216.0 & & 221.7 & & & \\
\hline$\%$ & $12: 00$ & 196.083 & & 362.9 & 338.8 & 355.3 & 355.0 & 336.9 & 348.4 & & & & 336.6 & 334.8 & 287.0 & & 309.8 & & & \\
\hline $174=6$ & $0: 00$ & 196.683 & & 166.1 & 229.4 & 222.2 & 216.1 & 199.3 & 212.4 & & & & 173.4 & 228.3 & 215.0 & & 229.3 & & & \\
\hline $14 \%$ & $12: 00$ & 197.083 & & 353.5 & 330.0 & 358.0 & 359.4 & 337.9 & 353.0 & & & & 337.7 & 328.0 & 288.0 & & 310.0 & & & \\
\hline $1 \%$. & $0: 00$ & 197.683 & & 152.3 & 223.4 & 217.1 & 210.1 & 192.4 & 204.7 & & & & 165.6 & 218.5 & 222.9 & & 223.3 & & & \\
\hline 1\%4 & $12: 00$ & 198.083 & & 366.6 & 338.8 & 365.8 & 363.0 & 339.7 & 356.6 & & & & 343.0 & 336.8 & 291.6 & & 313.5 & & & \\
\hline 1214 & $0: 00$ & 108.583 & & 187.9 & 230.4 & 231.0 & 223.0 & 202.8 & 221.2 & & & & 176.9 & 225.5 & 217.7 & & 228.5 & & & \\
\hline $135 \%$ & $12: 00$ & 199.083 & & 359.7 & 332.7 & 358.1 & 358.7 & 339.6 & 353.2 & & & & 336.1 & 330.7 & 290.7 & & 310.1 & & & \\
\hline 140 & $0: 00$ & 199.583 & & 156.7 & 228.1 & 222.4 & 215.3 & 195.0 & 207.4 & & & & 164.0 & 221.1 & 217.0 & & 224.3 & & & \\
\hline $114 \%$ & $12: 00$ & 200.083 & & 368.2 & 345.6 & 365.8 & 362.9 & 347.4 & 359.9 & & & & 341.1 & 341.9 & 298.3 & & 318.9 & & & \\
\hline 148 & $0: 00$ & 200.583 & & 165.4 & 232.3 & 233.8 & 228.4 & 208.1 & 224.8 & & & & 168.5 & 225.5 & 225.8 & & 231.4 & & & \\
\hline 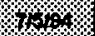 & $12: 00$ & 201.083 & & 361.7 & 348.0 & 372.3 & 368.5 & 346.7 & 364.7 & & & & 339.0 & 341.2 & 309.2 & & 318.2 & & & \\
\hline $1160 \%$ & $0: 00$ & 201.583 & & 144.7 & 225.3 & 223.4 & 218.1 & 196.7 & 215.4 & & & & 137.3 & 218.5 & 229.2 & & 222.8 & & & \\
\hline $1 \%$ & $12: 00$ & 202.063 & & 364.2 & 349.4 & 370.5 & 370.1 & 353.6 & 367.2 & & & & 320.7 & 344.7 & 321.2 & & 319.8 & & & \\
\hline $100 \%$ & \begin{tabular}{|l|}
$0: 00$ \\
\end{tabular} & 215.683 & & 354.3 & 367.3 & 380.4 & 358.4 & 345.5 & 433.1 & & & & 157.4 & 342.6 & 445.4 & & 304.5 & & & \\
\hline 160.4 & $12: 00$ & 216.083 & & 323.2 & 331.7 & 365.8 & 345.6 & 330.4 & 419.6 & & & & 149.4 & 303.0 & 456.3 & & 295.3 & & & \\
\hline $471 \%$ & $0: 00$ & 216.583 & & 381.4 & 374.6 & 391.1 & 363.0 & 354.3 & 443.0 & & & & 160.2 & 349.8 & 457.8 & & 313.5 & & & \\
\hline$\gamma \%$ & $12: 00$ & 217.083 & & 353.6 & 335.3 & 372.8 & 344.9 & 340.9 & 426.7 & & & & 151.2 & 303.8 & 464.2 & & 298.0 & & & \\
\hline 172814 & $0: 00$ & 217.683 & & 410.3 & 374.9 & 400.2 & 369.4 & 360.7 & 452.1 & & & & 162.2 & 345.7 & 487.8 & & 316.5 & & & \\
\hline $14 \% 9 \%$ & $12: 00$ & 218.083 & & 385.8 & 337.2 & 383.5 & 348.5 & 348.0 & 433.9 & & & & 151.4 & 302.3 & 471.4 & & 296.5 & & & \\
\hline $125 \%$ & $0: 00$ & 218.683 & & 436.9 & 374.7 & 408.6 & 367.5 & 365.0 & 454.4 & & & & 156.9 & 347.3 & 471.1 & & 311.1 & & & \\
\hline 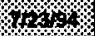 & $12: 00$ & 219.083 & & 421.3 & 340.6 & 392.1 & 351.8 & 354.9 & 440.7 & & & & 152.2 & 306.7 & 479.8 & & 298.9 & & & \\
\hline $10 \% \%$ & $0: 00$ & 219.583 & & 454.3 & 370.5 & 425.2 & 371.9 & 375.5 & 464.9 & & & & 158.7 & 337.8 & 481.6 & & 311.2 & & & \\
\hline 1204 & $12: 00$ & 220.083 & & 395.1 & 337.8 & 404.0 & 349.0 & 360.8 & 443.9 & & & & 148.6 & 303.0 & 484.8 & & 292.7 & & & \\
\hline 112140 & 0.00 & 220.683 & & 420.5 & 383.5 & 441.6 & 376.1 & 393.7 & 474.3 & & & & 155.2 & 353.4 & 485.7 & & 317.1 & & & \\
\hline 8) & $12: 00$ & 221.083 & & 160.4 & 347.6 & 431.2 & 360.5 & 389.8 & 463.2 & & & & 148.7 & 312.8 & 491.2 & 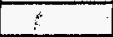 & 300.7 & & & \\
\hline$\%$ \% & $0: 00$ & 221.583 & & 288.1 & 385.3 & 459.8 & 381.3 & 420.8 & 485.6 & & & & 151.7 & 355.3 & 491.0 & & 317.2 & & & \\
\hline $5 \% 1 \%$ & 12:00 & 222.083 & & & 353.6 & 451.1 & 370.8 & 420.4 & 471.8 & & & & 153.0 & 317.2 & 497.2 & $\therefore$ & 304.1 & & & \\
\hline 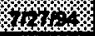 & $0: 00$ & 222.683 & & 263.8 & 378.3 & 476.3 & 388.2 & 448.7 & 496.0 & & & & 151.7 & 347.4 & 494.4 & & 312.8 & & & \\
\hline
\end{tabular}


Table 5. Tensiometer Data

\begin{tabular}{|c|c|c|c|c|c|c|c|c|c|c|c|c|c|c|c|c|c|c|c|c|}
\hline & & & & & & & & & Ten & slometer & Data & & & & & & & & & \\
\hline & & Test & $\begin{array}{c}\text { Soll } \\
\text { Moisture } \\
\text { Tension } \\
\left(\mathrm{Cm} \mathrm{H}_{2} \mathrm{O}\right)\end{array}$ & $\begin{array}{c}\text { Soll } \\
\text { Moisture } \\
\text { Tension } \\
\text { (cm Hz) } \\
\end{array}$ & $\begin{array}{c}\text { Soll } \\
\text { Molsture } \\
\text { Tension } \\
\left(\mathrm{C} \mathrm{H}_{2} \mathrm{O}\right) \\
\end{array}$ & $\begin{array}{c}\text { Soll } \\
\text { Molsture } \\
\text { Tenslon } \\
\left(\mathrm{Cm} \mathrm{H}_{2} \mathrm{O}\right) \\
\end{array}$ & 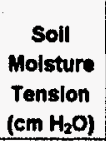 & $\begin{array}{c}\text { Soll } \\
\text { Moisture } \\
\text { Tension } \\
\left(\mathrm{cm} \mathrm{H}_{2} \mathrm{O}\right) \\
\end{array}$ & $\begin{array}{c}\text { Soll } \\
\text { Molsture } \\
\text { Tension } \\
\left.\text { (em } \mathrm{H}_{2} \mathrm{O}\right) \\
\end{array}$ & $\begin{array}{c}\text { Soll } \\
\text { Moisture } \\
\text { Tenslon } \\
\left(\mathrm{Cm} \mathrm{H}_{2} \mathrm{O}\right) \\
\end{array}$ & $\begin{array}{c}\text { Soll } \\
\text { Molsture } \\
\text { Tension } \\
\left(\mathrm{Cm} \mathrm{H}_{2} \mathrm{O}\right) \\
\end{array}$ & $\begin{array}{c}\text { Soll } \\
\text { Molsture } \\
\text { Tension } \\
\left.\text { (cm } \mathrm{H}_{2} \mathrm{O}\right) \\
\end{array}$ & $\begin{array}{c}\text { Soll } \\
\text { Moisture } \\
\text { Tension } \\
\left(\mathrm{Cm} \mathrm{H}_{2} \mathrm{O}\right) \\
\end{array}$ & $\begin{array}{c}\text { Soll } \\
\text { Molsture } \\
\text { Tension } \\
\left.\text { (em } \mathrm{H}_{2} \mathrm{O}\right) \\
\end{array}$ & 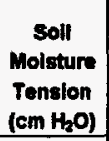 & $\begin{array}{c}\text { Soll } \\
\text { Moisture } \\
\text { Tension } \\
\text { (cm HzO) } \\
\end{array}$ & $\begin{array}{c}\text { Soll } \\
\text { Molsture } \\
\text { Tension } \\
\left(\mathrm{Cm}_{2} \mathrm{O} \text { ) }\right. \\
\end{array}$ & $\begin{array}{c}\text { Soll } \\
\text { molsture } \\
\text { Tension } \\
\left(\mathrm{cm} \mathrm{H}_{2} \mathrm{O}\right)\end{array}$ & $\begin{array}{c}\text { Soll } \\
\text { Molsture } \\
\text { Tenslon } \\
\left(\mathrm{Cm} \mathrm{H}_{2} \mathrm{O}\right) \\
\end{array}$ & $\begin{array}{c}\text { Soll } \\
\text { Moisture } \\
\text { Tonslon } \\
\left(\mathrm{cm} \mathrm{H}_{2} \mathrm{O}\right) \\
\end{array}$ \\
\hline Date & Time & Days $^{4}$ & $C 1^{\prime \prime}$ & $B 1^{2}$ & $A 1^{\prime \prime}$ & $\mathrm{C2}^{\mathrm{b}}$ & $\mathbf{B 2}^{\mathrm{b}}$ & $A 2^{b}$ & $C 3^{\mathrm{C}}$ & $B 3^{C}$ & $A 3^{c}$ & $\mathrm{CA}^{\mathrm{d}}$ & $\mathbf{B 4}^{\mathrm{d}}$ & $A 4^{d}$ & $C 5^{\circ}$ & $85^{\circ}$ & $A 5^{\circ}$ & $\mathrm{CG}^{\prime}$ & $B 6^{1}$ & $A 6^{\prime}$ \\
\hline 727 & $12: 00$ & 223.083 & & 129.2 & 361.5 & 480.7 & 392.4 & 401.4 & 498.6 & & & & 157.4 & 325.1 & 505.8 & & 309.3 & & & \\
\hline 1.1. & $2: 12$ & 244.675 & & & 139.5 & 654.0 & 97.8 & 652.0 & 603.0 & & & & 180.0 & 299.3 & 540.0 & & 238.2 & & & \\
\hline 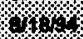 & $2: 14$ & 244.676 & & & 140.3 & 656.0 & 98.8 & 653.0 & 604.7 & & & & 160.0 & 300.2 & 539.8 & & 230.0 & & & \\
\hline 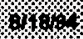 & 2:16 & 244.678 & & & 140.3 & 654.0 & 98.8 & 652.0 & 603.8 & & & & 180.0 & 300.1 & 539.9 & & 239.9 & & & \\
\hline 1040 & $2: 18$ & 244.679 & & & 142.8 & 655.0 & 97.9 & 852.0 & 603.8 & & & & 160.8 & 300.1 & 539.9 & & 239.8 & & & \\
\hline $8 \%$ & $2: 20$ & 244.681 & & & 142.8 & 653.0 & 88.8 & 652.0 & 603.8 & & & & 158.9 & 301.0 & 539.8 & & 239.9 & & & \\
\hline $8148 \mathrm{~s}$ & $2: 22$ & 244.682 & & & 142.8 & 855.0 & 98.7 & 651.0 & 603.8 & & & & 160.8 & 301.8 & 539.8 & & 241.6 & & & \\
\hline 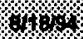 & $12: 00$ & 215.083 & & & 133.8 & 659.0 & 109.5 & 658.0 & 620.6 & & & & 172.0 & 349.7 & 536.6 & & 200.1 & & & \\
\hline $4 \%$ & $0: 00$ & 245.683 & & & 933.8 & 658.0 & 112.1 & 656.0 & 607.4 & & & & 171.1 & 282.9 & 540.9 & & 228.8 & & & \\
\hline 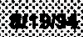 & $12: 00$ & 246.083 & & & 142.0 & 658.0 & 121.1 & 682.0 & 618.6 & & & & 178.6 & 324.4 & 538.1 & & 263.4 & & & \\
\hline 1209 & $0: 00$ & 246.583 & & & 140.1 & 655.0 & 120.0 & 651.0 & 601.1 & & & & 173.1 & 253.2 & 540.6 & & 198.1 & & & \\
\hline $8 \%$ & $12: 00$ & 247.083 & & & 150.8 & 659.0 & 131.5 & 666.0 & 614.8 & & & & 184.8 & 310.4 & 539.9 & & 251.3 & & & \\
\hline \% & $0: 00$ & 247.683 & & & 141.8 & 652.0 & 127.9 & 660.0 & 590.4 & & & & 177.7 & 248.1 & 541.2 & & 185.8 & & & \\
\hline 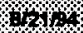 & $12: 00$ & 248.083 & & & 149.1 & 654.0 & 137.6 & 650.0 & 607.6 & & & & 184.1 & 298.4 & 534.1 & & 237.7 & & & \\
\hline 282344 & $0: 00$ & 248.683 & & & 145.4 & 647.0 & 134.0 & 681.0 & 582.6 & & & & 177.7 & 242.9 & 536.0 & & 188.0 & & & \\
\hline 18.4 & $12: 00$ & 249.083 & & & 154.3 & 650.0 & 137.8 & 682.0 & 598.0 & & & & 184.1 & 298.4 & 531.5 & & 235.0 & & & \\
\hline 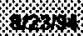 & $0: 00$ & 249.583 & & & 148.8 & 648.0 & 138.3 & 684.0 & 572.1 & & & & 181.1 & 239.4 & 534.1 & & 184.5 & & & \\
\hline 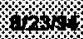 & $12: 00$ & 250.083 & & & 162.8 & 650.0 & 139.2 & 800.0 & 592.2 & & & & 187.3 & 300.8 & 533.5 & & 235.6 & & & \\
\hline 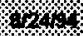 & $0: 00$ & 250.683 & & & 154.9 & 648.0 & 141.6 & 685.0 & 568.1 & & & & 184.4 & 238.4 & 532.0 & & 182.5 & & & \\
\hline $12 \%$ & $12: 00$ & 251.083 & & & 170.0 & 654.0 & 150.5 & 887.0 & 590.9 & & & & 194.4 & 296.5 & 533.0 & & 232.3 & & & \\
\hline \%zs\% & $0: 00$ & 251.683 & & & 459.3 & 646.0 & 147.6 & 668.0 & 557.0 & & & & 185.3 & 235.8 & 529.5 & & 177.4 & & & \\
\hline 82.8 & $12: 00$ & 252.083 & & & 178.5 & 647.0 & 144.2 & 649.0 & 579.7 & & & & 189.7 & 303.2 & 527.1 & & 235.4 & & & \\
\hline 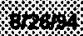 & $0: 00$ & 252.603 & & & 162.7 & 642.0 & 144.1 & 687.0 & 547.1 & & & & 184.2 & 240.0 & 523.8 & & 178.9 & & & \\
\hline (6. & $12: 00$ & 263.083 & & & 186.4 & 644.0 & 145.1 & 669.0 & 571.0 & & & & 192.3 & 308.5 & 473.5 & & 240.5 & & & \\
\hline 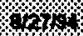 & $0: 00$ & 263.583 & & & 173.2 & 640.0 & 145.9 & 666.0 & 533.5 & & & & 187.0 & 253.3 & 522.5 & & 189.4 & & & \\
\hline$\%$, & $12: 00$ & 254.083 & & & 195.8 & 641.0 & 141.6 & 665.0 & 562.1 & & & & 192.2 & 313.7 & 517.3 & & 243.8 & & & \\
\hline $2 \%$ & $0: 00$ & 264.683 & & & 172.2 & 636.8 & 142.3 & 667.0 & 520.1 & & & & 187.7 & 256.7 & 516.0 & & 191.0 & & & \\
\hline 14\% & $12: 00$ & 265.083 & & & 195.8 & 637.2 & 139.0 & 684.0 & 551.7 & & & & 192.2 & 317.2 & 512.0 & & 245.5 & & & \\
\hline 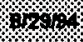 & $0: 00$ & 255.683 & & & 173.0 & 631.4 & 137.9 & 667.0 & 508.7 & & & & 186.7 & 254.8 & 510.6 & & 189.1 & & & \\
\hline 182. 64 & $12: 00$ & 266.083 & & & 198.5 & 634.6 & 134.7 & 657,0 & 541.3 & & & & 192.2 & 313.7 & 505.2 & & 242.1 & & & \\
\hline $58.0 \%$ & 0.00 & 266.583 & & & 157.8 & 612.4 & & 627.6 & 494.9 & & & & 155.6 & 163.2 & 385.1 & & 80.2 & & & \\
\hline $18,0 \%$ & $12: 00$ & 257.083 & & & 190.5 & 587.5 & 119.2 & 818.4 & 490.8 & & & & 174.0 & 296.7 & 463.7 & & 219.0 & & & \\
\hline 패 & 1:01 & 273.026 & & 192.2 & 272.3 & 337.8 & & & & & & & & 100.8 & 253.3 & & 257.2 & & & \\
\hline 4 & $1: 02$ & 273.026 & & 192.2 & 273.2 & 337.0 & & & & & & & & 100.8 & 251.6 & & 258.3 & & & \\
\hline 44 & 1.03 & 273.627 & & 191.4 & 272.3 & 334.4 & & & & & & & & 102.8 & 250.7 & & 256.3 & & & \\
\hline 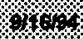 & $1: 04$ & 273.628 & & 189.6 & 272.3 & 334.4 & & & & & & & & 104.3 & 250.7 & & 254.6 & & & \\
\hline is. & $1: 05$ & 273.628 & & 189.6 & 272.3 & 333.5 & & & & & & & & 104.3 & 250.7 & & 254.5 & & & \\
\hline 84 (1) & 1:06 & 273.620 & & 189.6 & 273.1 & 334.4 & & & & & & & & 108.9 & 253.2 & & 253.7 & & & \\
\hline
\end{tabular}


Table 5. Tensiometer Data

\begin{tabular}{|c|c|c|c|c|c|c|c|c|c|c|c|c|c|c|c|c|c|c|c|c|}
\hline & & & & & & & & & \multicolumn{3}{|c|}{ Tensiometer Data } & & & & & & & & & \\
\hline & & Test & $\begin{array}{c}\text { Soll } \\
\text { Molsture } \\
\text { Tension } \\
\left.\text { (cm } \mathrm{H}_{2} \mathrm{O}\right) \\
\end{array}$ & $\begin{array}{c}\text { Soll } \\
\text { Moisture } \\
\text { Tansion } \\
\left(\mathrm{Cm} \mathrm{H}_{2} \mathrm{O}\right) \\
\end{array}$ & $\begin{array}{c}\text { Soll } \\
\text { Molsture } \\
\text { Tension } \\
\left(\mathrm{cm} \mathrm{H}_{2} \mathrm{O} \text { ) }\right. \\
\end{array}$ & $\begin{array}{c}\text { Soll } \\
\text { Moisture } \\
\text { Tension } \\
\left(\mathrm{cm} \mathrm{H}_{2} \mathrm{O}\right) \\
\end{array}$ & $\begin{array}{c}\text { Soll } \\
\text { Moisture } \\
\text { Tenslon } \\
\left(\mathrm{Cm} \mathrm{H}_{2} \mathrm{O}\right) \\
\end{array}$ & $\begin{array}{c}\text { Soll } \\
\text { Molsture } \\
\text { Tension } \\
\left(\mathrm{Cm} \mathrm{H}_{2} \mathrm{O}\right) \\
\end{array}$ & $\begin{array}{c}\text { Soll } \\
\text { Molsture } \\
\text { Tension } \\
\left(\mathrm{cm} \mathrm{H}_{2} \mathrm{O}\right) \\
\end{array}$ & $\begin{array}{c}\text { Soll } \\
\text { Moisture } \\
\text { Tension } \\
\left(\mathrm{Cm} \mathrm{H}_{2} \mathrm{O}\right) \\
\end{array}$ & $\begin{array}{c}\text { Soll } \\
\text { Moisture } \\
\text { Tension } \\
\left(\mathrm{cm} \mathrm{H}_{2} \mathrm{O}\right) \\
\end{array}$ & $\begin{array}{c}\text { Soil } \\
\text { Moisture } \\
\text { Tension } \\
\left(\mathrm{Cm}_{2} \mathrm{O}\right) \\
\end{array}$ & $\begin{array}{c}\text { Soll } \\
\text { Moisture } \\
\text { Tenslon } \\
\left(\mathrm{cm} \mathrm{H}_{2} \mathrm{O}\right) \\
\end{array}$ & $\begin{array}{c}\text { Soll } \\
\text { Moisture } \\
\text { Tension } \\
\left(\mathrm{Cm} \mathrm{H}_{2} \mathrm{O}\right) \\
\end{array}$ & $\begin{array}{c}\text { Soll } \\
\text { Moisture } \\
\text { Tension } \\
\left(\mathrm{em} \mathrm{H}_{2} \mathrm{O}\right) \\
\end{array}$ & $\begin{array}{c}\text { Soll } \\
\text { Molsture } \\
\text { Tension } \\
\left.\text { (em } \mathrm{H}_{2} \mathrm{O}\right) \\
\end{array}$ & 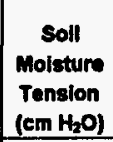 & $\begin{array}{c}\text { Soll } \\
\text { Molsture } \\
\text { Tenslon } \\
\text { (em Hz) } \\
\end{array}$ & $\begin{array}{c}\text { Soll } \\
\text { Molsture } \\
\text { Tenslon } \\
\left(\mathrm{cm} \mathrm{H}_{2} \mathrm{O}\right)\end{array}$ & $\begin{array}{c}\text { Soll } \\
\text { Molsture } \\
\text { Tension } \\
\left(\mathrm{Cm} \mathrm{H}_{2} \mathrm{O}\right)\end{array}$ \\
\hline Date & Time & Days ${ }^{1}$ & $\mathrm{Cl}^{\circ}$ & $B 1^{*}$ & $\mathrm{A1}^{\circ}$ & $\mathrm{C2}^{\mathrm{b}}$ & $B 2^{b}$ & $A 2^{6}$ & $\mathrm{C3}^{\mathrm{c}}$ & $\mathbf{B 3}^{\mathbf{c}}$ & $\mathrm{A3}^{\mathrm{C}}$ & $\mathrm{CA}^{\mathrm{d}}$ & $B 4^{d}$ & $\mathrm{A4}^{\mathrm{d}}$ & $C 5^{\circ}$ & $B 5^{\circ}$ & $A 5^{\circ}$ & $\mathrm{Co}^{\prime}$ & $\mathrm{BG}^{\mathrm{f}}$ & $A 6^{f}$ \\
\hline$\% \%$ & 22:13 & 288.509 & & 142.8 & 132.8 & 387.9 & 204.0 & & & & & & & & 268.8 & & & 223.5 & & \\
\hline$\%$ & 22:14 & 288.610 & & 141.8 & 132.8 & 387.9 & 204.9 & & & & & & & & 286.8 & & & 223.5 & & \\
\hline $0110 \%$ & $0: 01$ & 305.684 & & 193.3 & 140.7 & 367.7 & & 384.3 & & & & & 198.0 & & 355.0 & & & 184.5 & & \\
\hline $1 \%$ & $0: 02$ & 305.685 & & 193.3 & 140.7 & 367.7 & & 382.5 & & & & & 195.1 & & 355.0 & & & 184.5 & & \\
\hline $10 \%$ & 0.03 & 306.585 & & 193.3 & 140.7 & 367.7 & & 383.4 & & & & & 196.0 & & 354.1 & & & 185.4 & & \\
\hline $14 \%$ & $0: 11$ & 332.591 & & 301.2 & & 450.8 & & 532.8 & 438.7 & & & & & & 383.1 & & & & & \\
\hline \% & $0: 12$ & 332.592 & & 309.3 & & 379.4 & & 477.9 & 427.1 & & & & & & 545.3 & & & & & \\
\hline 114 & $0: 13$ & 332.592 & & 250.3 & & 385.6 & & 477.9 & 398.2 & & & & & & 348.6 & & & & & \\
\hline $11 \%$ & $0: 14$ & 332.503 & & 48.4 & & 508.3 & & 358.5 & 423.9 & & & & & & 338.5 & & & & & \\
\hline & & $\frac{a=\text { clust }}{d=\text { clust }}$ & $\frac{\operatorname{cs} A, B, 2}{\sin B, a}$ & $\frac{n d C \text { at } 1}{\text { ad } C \text { at } 4}$ & $\begin{array}{l}\text { it depth } \\
\text { it depth }\end{array}$ & $\begin{array}{l}b=\text { cluste } \\
e=\text { clust }\end{array}$ & $\frac{r s A_{1}, a}{r s A, B, a}$ & $\frac{n d C \text { at } 2}{\text { nd } C \text { at } 5}$ & $\begin{array}{l}\text { ft depth } \\
\text { ft depth }\end{array}$ & $\begin{array}{l}c=c \text { cluste } \\
f=\text { cluste }\end{array}$ & $\frac{r_{S} A, B, a}{r_{S} A, B, a}$ & $\frac{n d C \text { at } 3}{\text { nd } C \text { at } 6}$ & $\frac{\text { tt depth }}{\text { fi depth }}$ & & & & & & & \\
\hline
\end{tabular}




\subsection{Test Results}

\subsection{Neutron Moisture Meter Data}

Figures 7 and 8 present the volumetric moisture content data measured at the center and southeast corner access tubes. These plots show the volumetric moisture content as a function of time for each $30 \mathrm{~cm}(1 \mathrm{ft})$ depth interval within the IP test plot. The abscissa, labeled "Experiment Days", represents the number of days that drainage has occurred. Hence, $T_{0}$ corresponds to 10:40 AM on December 16, 1993, the time that the pump was shut off and the drainage phase of the test was initiated.

Figures 7 and 8 clearly show that at time $T_{0}$ the entire vertical profile is at or near field saturation, with volumetric moisture contents in the 29 to 35 percent range, and an average of 31.5 percent in the center of the IP plot. Moisture contents decreased rapidly initially, once the drainage phase of the test was initiated. This rapid drainage is shown by steep negative slopes of the curves during the first few days of the IP test, and reflects the greater hydraulic conductivities at near-saturated conditions.

Drainage diminishes rapidly after the first few days as hydraulic conductivities significantly decrease with decreasing moisture contents. By the first 25 days of the test, the increased drainage rates have diminished to a relatively constant rate, as indicated by the relatively flat, negative slopes of the curves. By January 1995, the average volumetric moisture content in the center of the IP test plot was 18.3 percent, and was decreasing at an estimated one percent every 100 days.

Intuitively, one might expect a clear wetting trend with depth at a given point in time, as water drains from the upper layers of the IP plot through the lower layer. This trend is generally true. As shown in Figures 7 and 8, the upper layers of the plot are generally drier than the lower layers. However, the trend is not absolute, perhaps due to vertical and horizontal heterogeneities within the plot.

For example, the moisture contents at the $30 \mathrm{~cm}(1 \mathrm{ft})$ depth in the SE corner were greater than moisture contents at the $180 \mathrm{~cm}(6 \mathrm{ft})$ depth. This is most likely attributable to differences in soil hydraulic properties at the various depths. These differences became more apparent later, as the IP data were analyzed, and variations in soil textural properties and particle size distributions were evaluated. 


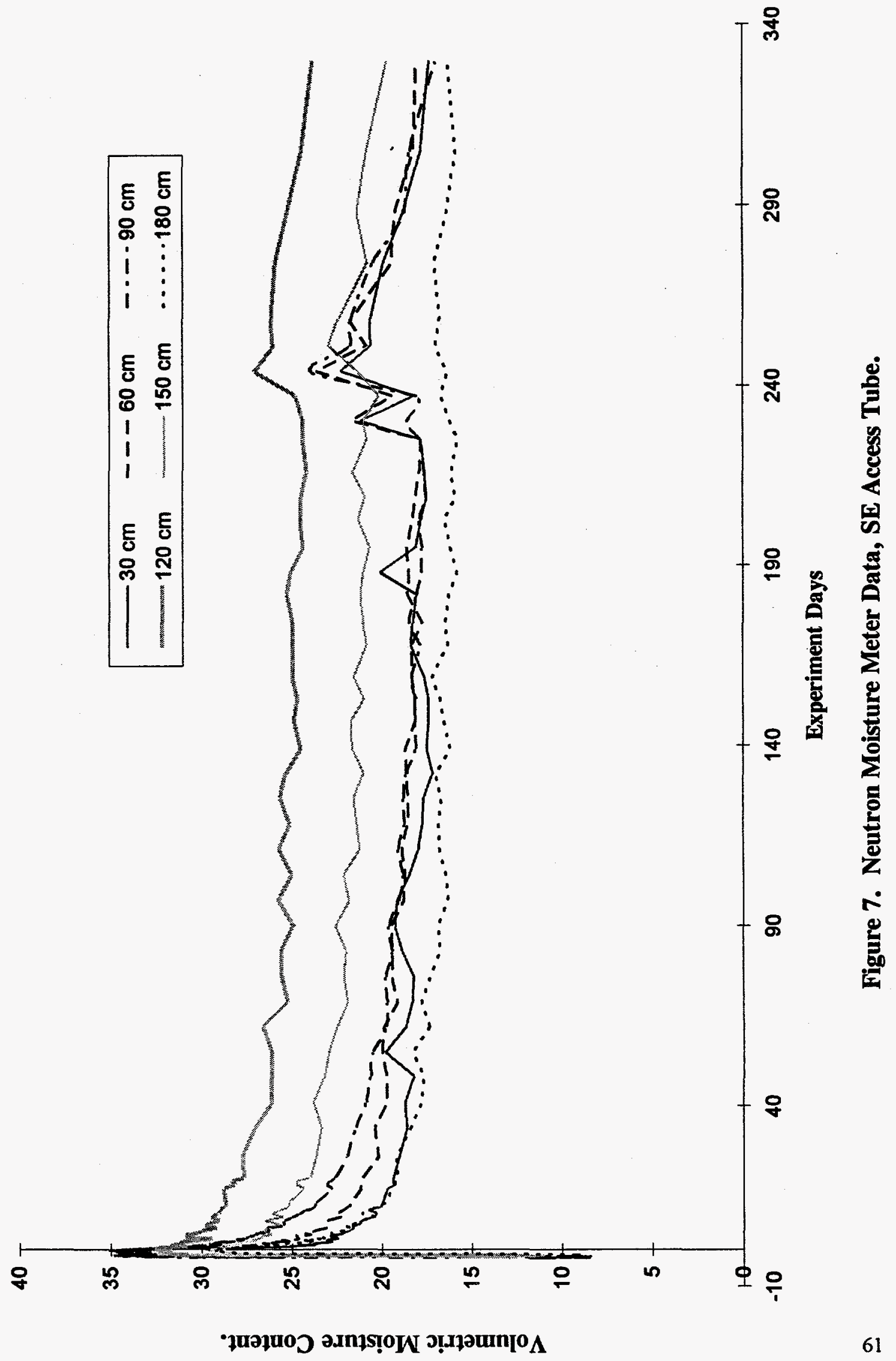




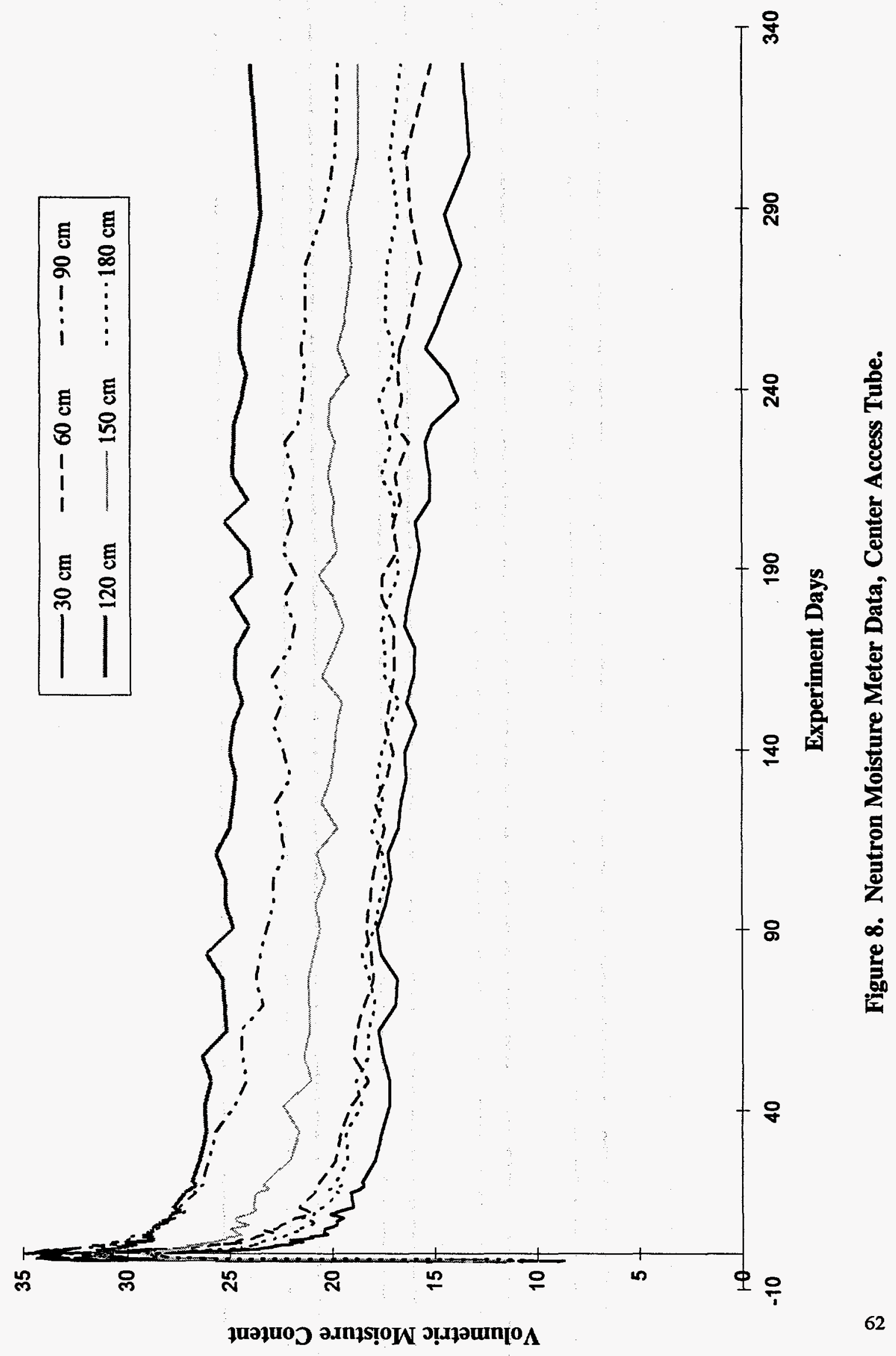




\subsection{Tensiometer Data}

Figures 9 through 14 show the soil tension values at each $30 \mathrm{~cm}(1 \mathrm{ft})$ depth interval for each instrument cluster (A, B, and C) within the IP plot. The soil tensions at all of the depths $(30 \mathrm{~cm}, 60 \mathrm{~cm}$, etc.) increase rapidly during the first few days of the drainage test. The rapid initial increase in tensions corresponded directly to the initial decrease in moisture contents discussed above. As the test progressed, the rate of increase in soil tensions at each depth diminished due to the decreased drainage rate.

Figures 9 through 14 also show a significant increased variability of tension values with time, particularly after the first 30 to 50 days. This variability is believed to be due to diurnal temperature fluctuations in the test plot. The first several months of the test were conducted through mid-February, when temperatures in the Albuquerque area were the lowest. Increased variability in soil tensions seem to correspond with the higher temperatures of spring and summer.

Approximately 200 days into the IP test, the data measurement frequency for the tensiometers was decreased to weekly, and later to monthly intervals, as the rate of drainage decreased from the IP test plot. Unfortunately, the reduced amount of data limits its usefulness during the later stages of the test.

The tensiometers were also considered somewhat problematic, and some failed as the months progressed. Some tensiometers were replaced as they failed, but the number of operational tensiometers decreased considerably towards the end of the test.

Finally, it appears that some tensiometers in the IP test plot have reached the limits of their operational range. The operational range of the tensiometers at Albuquerque's elevation is limited from $0 \mathrm{~cm}$ to approximately $600 \mathrm{~cm}(236 \mathrm{in})$. Figures 10,11 , and 13 suggest that tensiometers at depths of 60,90 , and $150 \mathrm{~cm}(2,3$, and $5 \mathrm{ft})$ may have reached their operational limits (i.e. soil tensions at these depths may have exceeded $600 \mathrm{~cm}$ ). Once tensions have exceeded this level, the readings provided by the tensiometers are erroneous, and other types of instrumentation (such as thermocouple psychrometers) must be used to measure soil tension. No thermocouple psychrometers were installed in the IP plot due to time and budget constraints. 


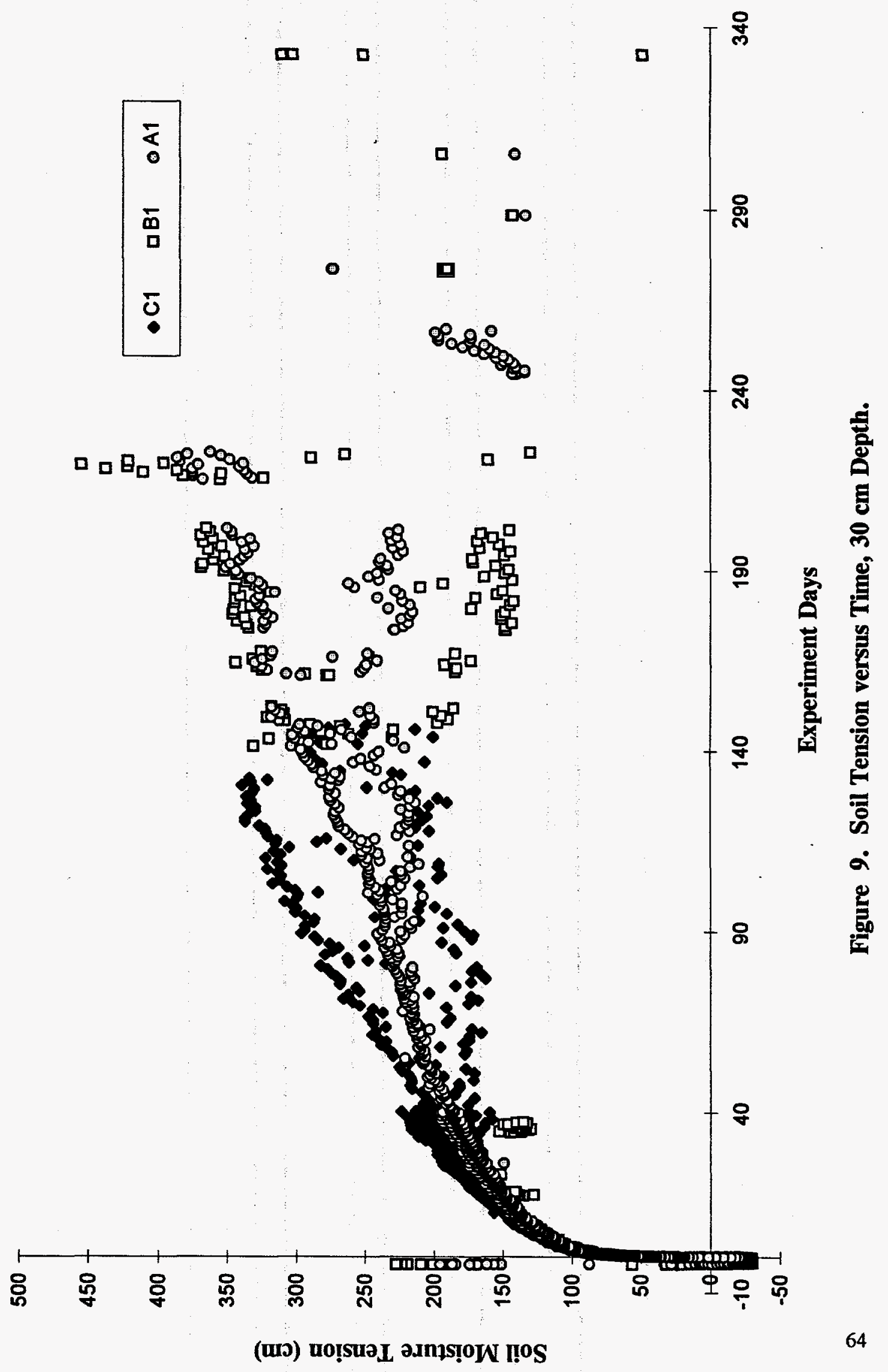




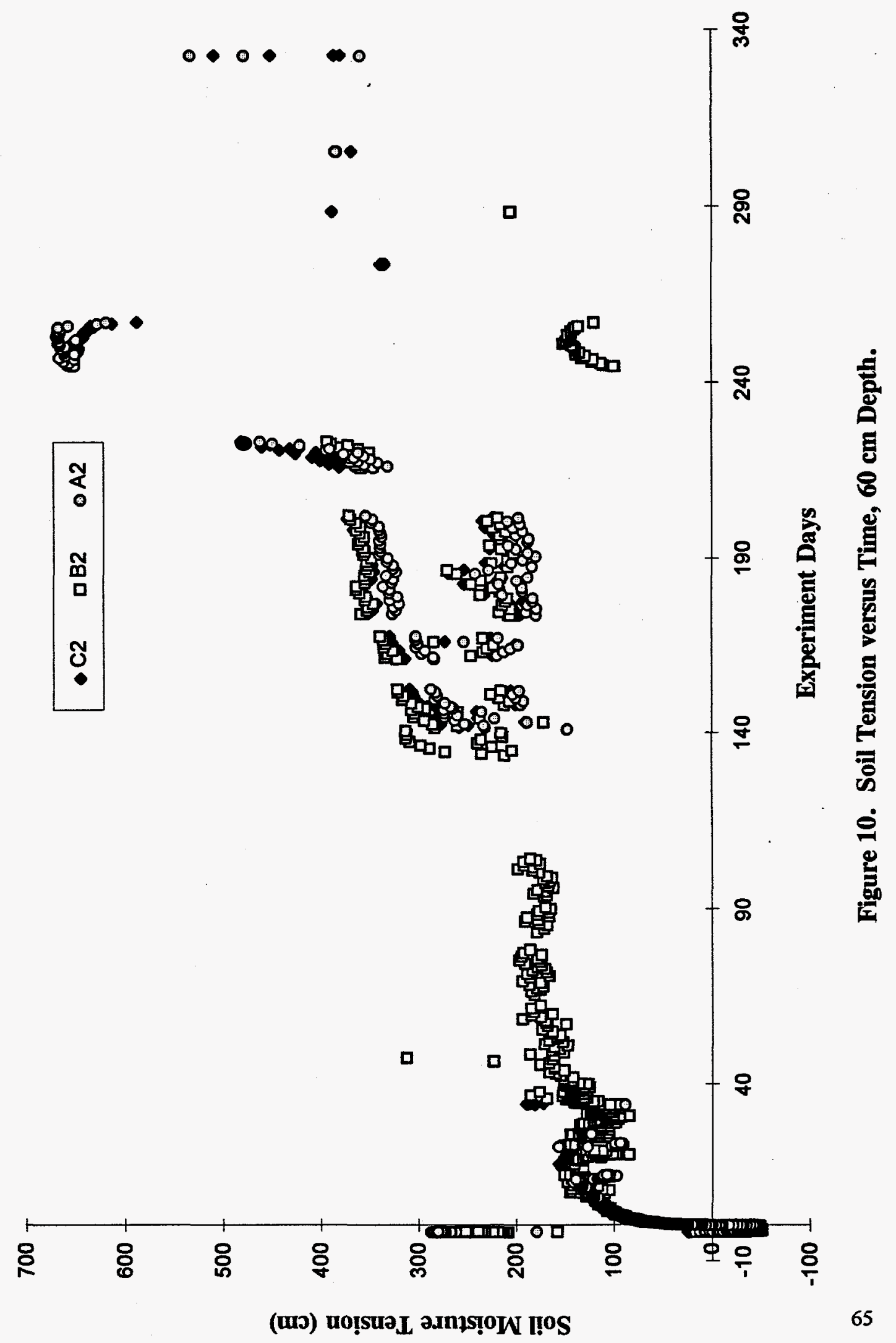




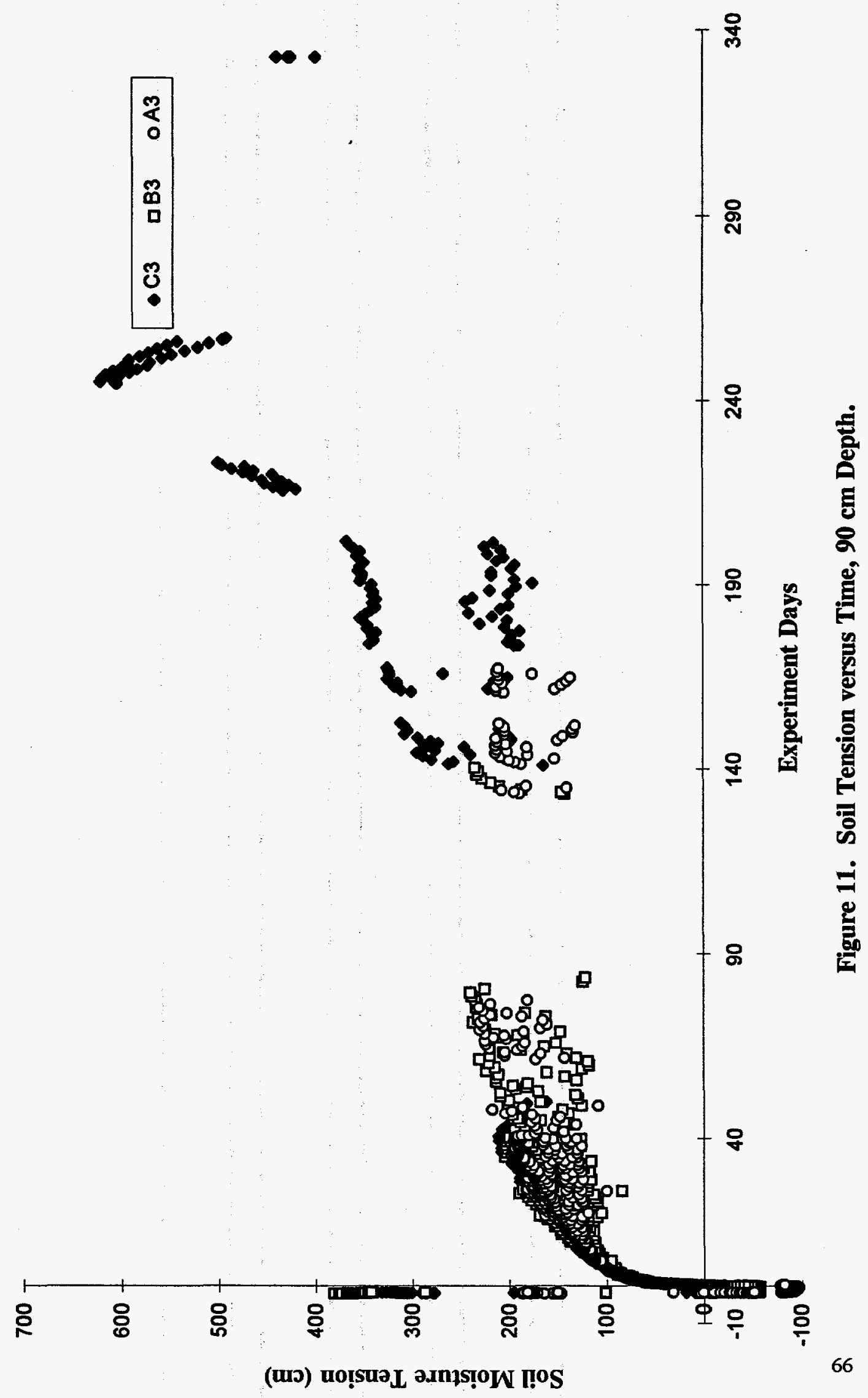




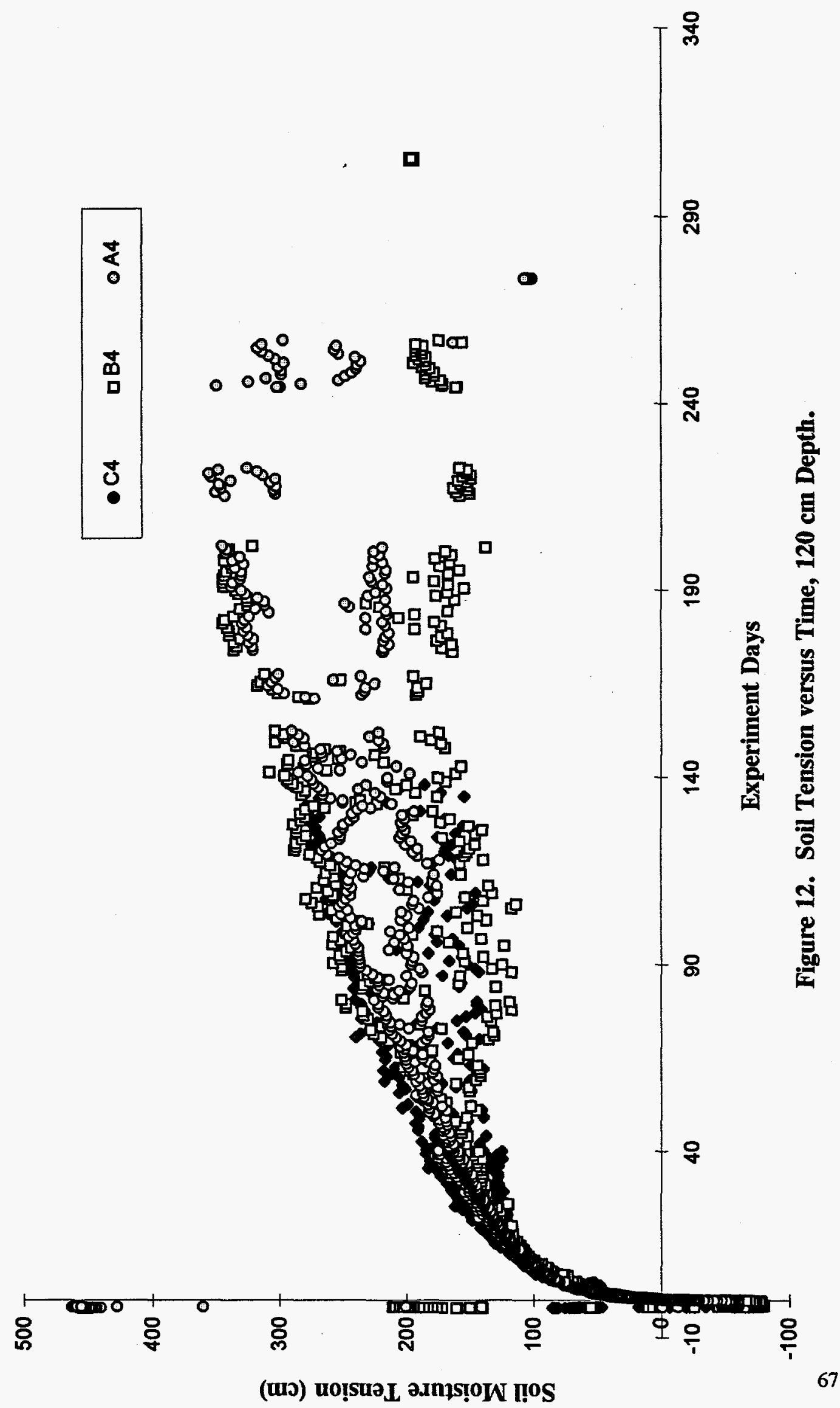




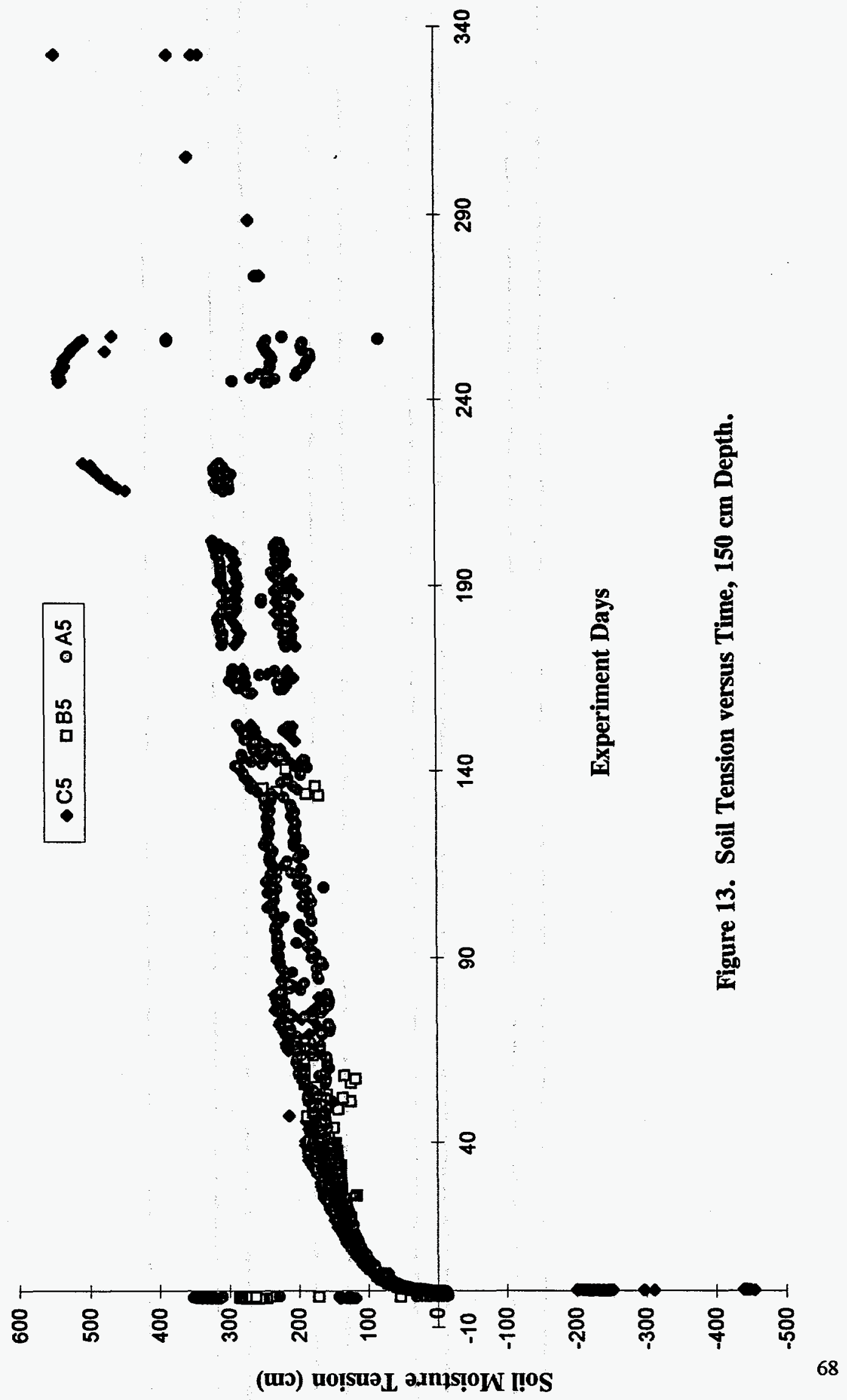




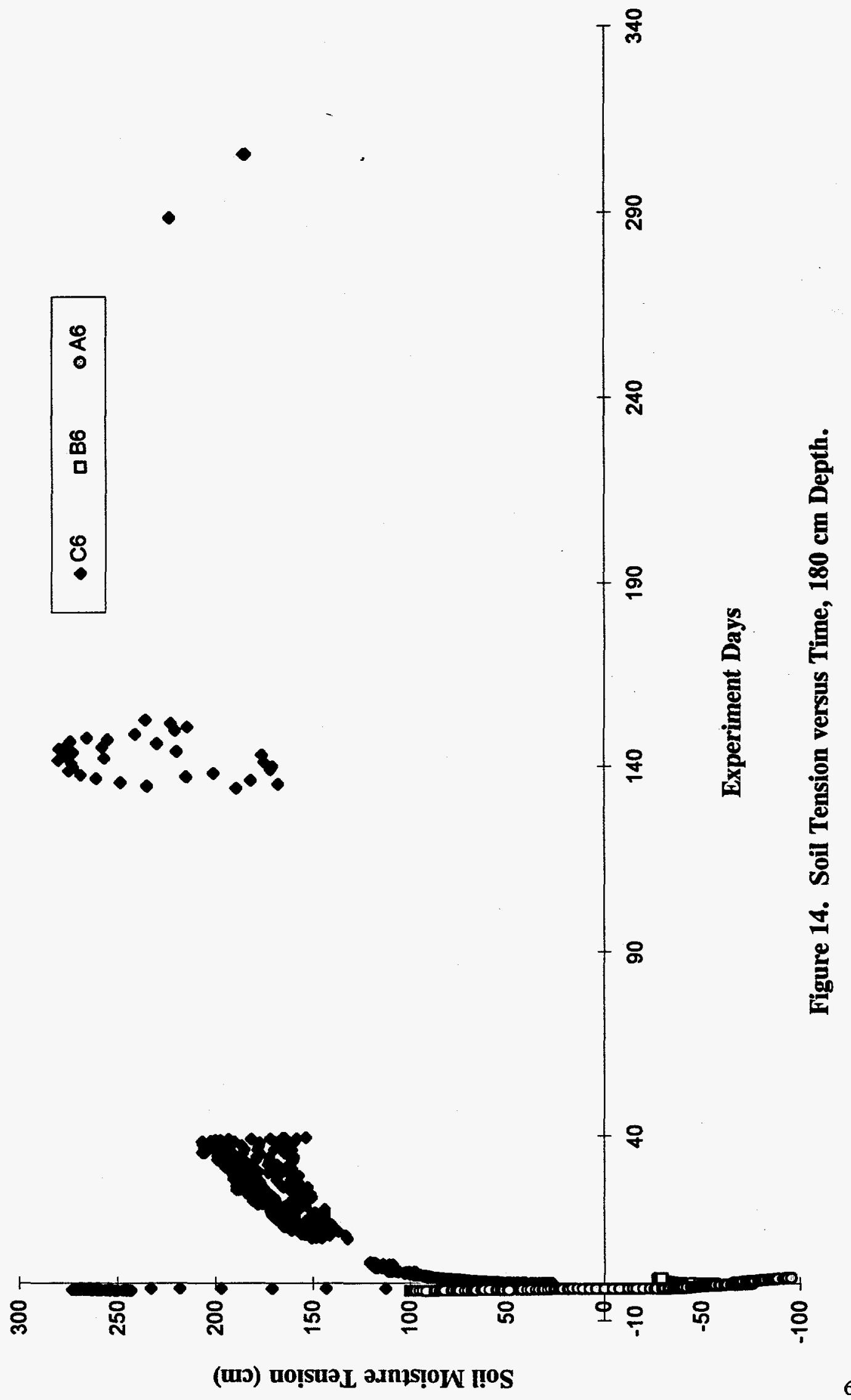




\subsection{Summary}

The moisture content and soil tension data presented in this report will be used to determine the unsaturated hydraulic properties of soils near of moisture content, and the soil moisture characteristics (the relationship between the MWL, including in situ measurements of unsaturated hydraulic conductivity as a function volumetric water content and soil tension).

The IP test was conducted in the silty sands of the Madurez Loamy Fine Sand Series, approximately $150 \mathrm{~m}(500 \mathrm{ft})$ west of the MWL. The results of the MWL IP test clearly demonstrate the influence of heterogeneities within the soil profile. The effects of these heterogeneities were observed both vertically and horizontally in the test plot.

Drainage from the IP test site was initially high during the first few days of the test, but significantly decreased after the first few weeks of the test. The significantly decreased rate of drainage during the latter part of the test reflects the low unsaturated hydraulic conductivities of the soils.

Initial conditions in the soil profile of the IP plot (prior to flooding) averaged 11.4 percent water by volume in the center of the test plot. After 20,300 liters (5494 gal.) of water were applied to the plot, volumetric moisture contents in the center of the test plot averaged 31.5 percent. As of January, 1995, the volumetric moisture contents averaged 18.3 percent, and are decreasing at an approximate rate of one percent (volumetric) every 100 days. Hence, the present drainage is extremely slow.

The in situ data obtained from this test is superior to laboratory measurements because there has been no disturbance of soil structure due to invasive sampling. The unsaturated hydraulic parameters determined from this test will be used for modeling unsaturated flow and contaminant transport from the MWL, and for reducing uncertainties in the risk assessment for the MWL. This information will provide a better understanding of the potential for contaminant migration from the MWL to groundwater, and the resulting risk to human health and the environment. Finally, the data collected from the IP test will be used to help evaluate remedial alternatives for the MWL. 


\section{References}

Hillel, D., V. Krentos, and Y. Stylianou., 1972, Procedure and Test of an Internal Drainage Method for Measuring Soil Hydraulic Characteristics In Situ, Soil Science, Vol. 114, pp. 395 - 400.

SNL/NM, 1993, Mixed Waste Landfill Phase 2 RCRA Facility Investigation Work Plan, prepared by Sandia National Laboratories Environmental Restoration Program for the U.S. Department of Energy, Albuquerque, New Mexico, 1993.

Ogata, G., and Richards, L.A., 1957, Water Content Changes Following Irrigation of Bare Field Soil that is Protected from Evaporation, Soil Science, Vol. 21, pp 355 - 356.

Research by Peter Wierenga (New Mexico State University) on the Mixed Waste Landfill (unpublished report). SNL/NM, 1980.

Richards, S.J., and Weeks, L.V., 1953, Capillary Conductivity Values from Moisture Yield and Tension Measurements on Soil Columns, Soil Science, Vol. 17, pp 206 - 209.

Soil Survey of Bernallilo County and Parts of Sandoval and Valencia Counties, New Mexico, United States Department of Agriculture - Soil Conservation Service, June 1977.

Watson, K. K., C. 1966, An Instantaneous Profile Method for Determining the Hydraulic Conductivity of Unsaturated Porous Media, Water Resources Research, Vol. 2, No. 4, pp. 709 - 715. 


\section{DISTRIBUTION}

1

4

Stephen C. Bayliss

DanShar, Inc.

1215 Chiquitos Rd.

Bosque Farms, NM 87068

$\begin{array}{lll}4 & \text { MS } 0184 & \text { John-Olav Johnsen, DOE/KAO } \\ 1 & \text { MS 0720 } & \text { R.G. Knowlton, 6626 } \\ 1 & \text { MS 1148 } & \text { R.E. Fate, 7585 } \\ 1 & \text { MS 1148 } & \text { J.L. Peace, 7585 } \\ 1 & \text { MS 1148 } & \text { T.J. Goering, 7585 } \\ 1 & \text { MS } 1148 & \text { M.D. McVey, 7585 } \\ 1 & \text { MS } 1132 & \text { W.R. Strong, 7584 } \\ & & \\ 1 & \text { MS } 1309 & \text { Environmental Operations Records Center, 7512 } \\ 1 & \text { MS } 9018 & \text { Central Technical Files, 8523-2 } \\ 1 & \text { MS 0619 } & \text { Print Media, 12615 } \\ 5 & \text { MS 0899 } & \text { Technical Library, 4414 } \\ 2 & \text { MS 0100 } & \text { Document Processing, 7613-2 } \\ & & \text { For DOE/OSTI }\end{array}$

